\title{
WestVirginiaUniversity
}

THE RESEARCH REPOSITORY @ WVU

Graduate Theses, Dissertations, and Problem Reports

2021

\section{Pulsar Noise Processes and Emission Physics}

Brent Jacob Shapiro-Albert

West Virginia University, bjs0024@mix.wvu.edu

Follow this and additional works at: https://researchrepository.wvu.edu/etd

Part of the Stars, Interstellar Medium and the Galaxy Commons

\section{Recommended Citation}

Shapiro-Albert, Brent Jacob, "Pulsar Noise Processes and Emission Physics" (2021). Graduate Theses, Dissertations, and Problem Reports. 8060.

https://researchrepository.wvu.edu/etd/8060

This Dissertation is protected by copyright and/or related rights. It has been brought to you by the The Research Repository @ WVU with permission from the rights-holder(s). You are free to use this Dissertation in any way that is permitted by the copyright and related rights legislation that applies to your use. For other uses you must obtain permission from the rights-holder(s) directly, unless additional rights are indicated by a Creative Commons license in the record and/ or on the work itself. This Dissertation has been accepted for inclusion in WVU Graduate Theses, Dissertations, and Problem Reports collection by an authorized administrator of The Research Repository @ WVU.

For more information, please contact researchrepository@mail.wvu.edu. 
Graduate Theses, Dissertations, and Problem Reports

2021

Pulsar Noise Processes and Emission Physics

Brent Jacob Shapiro-Albert

Follow this and additional works at: https://researchrepository.wvu.edu/etd

Part of the Stars, Interstellar Medium and the Galaxy Commons 


\title{
Pulsar Noise Processes and Emission Physics
}

\author{
Brent J. Shapiro-Albert
}

\author{
Dissertation Submitted to \\ The Eberly College of Arts and Sciences \\ at West Virginia University \\ in partial fulfillment of the requirements \\ for the degree of \\ Doctor of Philosophy \\ in \\ Physics
Maura McLaughlin, Ph.D., Chair
Duncan Lorimer, Ph.D.
Loren Anderson, Ph.D.
Kevin Bandura, Ph.D. \\ Department of Physics and Astronomy \\ Morgantown, West Virginia, USA \\ 2021
}

Keywords: pulsars, interstellar medium

Copyright 2021 Brent J. Shapiro-Albert 
Abstract

\title{
Pulsar Noise Processes and Emission Physics
}

\author{
Brent J. Shapiro-Albert
}

Precision pulsar timing can be used to study many different astrophysically interesting phenomena, from the emission mechanism of pulsars to the detection of nanohertz gravitational waves. These analyses span topics such as studying the single pulses of pulsars and analyzing years of pulsar timing data from pulsar timing arrays (PTAs). Single-pulse studies allow us to glean information on the emission physics of pulsars on their shortest timescales, while PTA observations of millisecond pulsars (MSPs) allow us to not only study the pulsars themselves, but also probe the interstellar medium (ISM) and constrain the noise in the data for precision pulsar timing experiments.

To study the emission properties of pulsars, we compiled a large population of single pulses from three rotating radio transients (RRATs), from which we detect sporadic, but periodic pulsations and whose emission mechanisms remain largely unknown. Our study found that the average spectral indices of these RRATs is flatter than most pulsars, with a power-law index of $\alpha=-0.9$, but that the distribution of single-pulse spectral indices is large. We also find that the single-pulse flux distributions of these three RRATs generally follow a log-normal distribution, suggesting the detected radio emission is not due to giant pulses. Further we find that single-pulse flux is not correlated with the wait-time between pulses, and thus is not produced by mechanisms such as the storage and release of energy in the pulsar magnetosphere.

We then analyze multi-hour continuous observations of seven MSPs in the North American Nanohertz Observatory for Gravitational Wave (NANOGrav) PTA to characterize the ISM along their lines-of-sight. These unique observations allowed us to place some of the best limits on the scintillation bandwidth and timescale for these MSPs, some for the first time, and show that the root mean square (rms) noise due to scattering in these MSPs is $\lesssim 50$ ns. We further showed that, as expected, the dispersion measure (DM) of these MSPs does not vary on $\sim$ hour long timescales, and that the timing precision of these MSPs does not decrease if the observations are not contiguous, as expected for observations by telescopes like the Canadian Hydrogen Intensity Mapping Experiment.

Finally, we explore the covariances between various radio frequency-dependent parameters in pulsar timing models and how they contribute to the overall noise budget of the PTA. To do this, we developed the Pulsar Signal Simulator Python package and use it to generate simulated data sets for three NANOGrav MSPs. We find a clear correlation between the mean injected scattering timescale and the spread in the recovered DM value, with larger scattering timescales corresponding to larger spreads. However, we find that this covariance, while important to quantifying the noise budget of the PTA, does not affect the timing precision of the simulated MSPs. 


\section{Dedication}

In honor of my grandpa, Allan Shapiro, for showing me how to be the best person I can be, and so much more. 


\section{Acknowledgements}

There are many people that deserve thanks for all of the work they put in to get me to this point. First I would like to thank my advisor Maura McLaughlin for all of the work and support she has given me over the last five years. Not only has she helped me to grow as a scientist, but also as a better, well-rounded person by seeing the value and encouraging me in non-research related projects.

A huge thank you to all of my undergraduate professors who helped get me here. In particular Jon Marr, who taught me what radio astronomy is and gave me my first research opportunity, Jim Cordes and Shami Chatterjee, who taught me what pulsars are, and Greg Hallenbeck, who helped me become a better scientist and showed me I had what it takes to be an astronomer.

I would also like to thank the members of the NANOGrav collaboration for teaching me a truly massive amount over the past few years. In particular, I would like to thank Michael Lam, Jeff Hazboun, Megan Jones, Joe Swiggum, and Paul Baker for not only teaching me, but serving as mentors and role models.

I certainly could not have done this without all of my many friends at WVU, particularly Andrew Kaiser, Evan Smith, and Will Fiore. Not only did you all go out of your way to help me when I needed it, but you always supported me, commiserated with me, drank with me, brought me into your D\&D campaigns, and rallied with me when the need arose. You truly made this experience bearable and I am forever grateful for your friendship. Further, I would like to thank PAGSO and all of the people who have served as active members and officers over the years who have put in an immense amount of work into making this department a better place, and improving the lives of graduate students, regardless of the obstacles in your way.

The biggest thank you of all must of course go to Caitlin Witt. Caitlin, I certainly would not be without all of your help and support over the last five years. Not only did you drag me (occasionally kicking and screaming) through classes and exams, you were always there to help me prepare and study. You have supported me through tough times and celebrated with me for even the smallest victories. I could not have done this without you, nor would I have wanted to. I am truly lucky to have you in my life, thank so you much for everything.

I of course also need to thank my (incredibly large) family. My parents, Liz Shapiro, Steve Albert, Steve Greider, and Janie Goldenberg, my siblings Elena Shapiro-Albert and Mitch Shapiro-Albert, and my grandparents, Allan and Carol Shapiro and Dan and Ellie Albert, have all given me unconditional support and love, and always asked me what I was working on, even if they knew they wouldn't understand it. I could not have made it here without your help. And of course, to the rest of my family, aunts, uncles, and cousins, for being so supportive. You have all worked so hard to help me become the person I am today and I will be forever grateful for that.

Many of my friends also deserve a big thank you for helping me get to this point. Matt Belikov and Che Chengsupanamit for always believing in me and for unconditional support and love. Dan Waldman for always sticking by my side no 
matter how bad a decision I was about to make was. Shauna LeFebvre, Becca Krasa, Julia Isaac, and Miranda Waldman for trying to prevent me from making those bad decisions, but supporting me despite them. Harry Hausner, Michael Warrener, and Ryan Muther for dragging me through my undergraduate physics and math classes. All you have believed in me even when I didn't believe in myself.

Finally, I could not possibly leave off Grace and Steve at the Apothecary, and Amber at Chestnut, for providing me with the fuel I needed to get make it through grad school. Thank you all. 


\section{Table of Contents}

List of Tables $\quad$ ix

List of Figures $\quad$ X

1 Introduction 1

1.1 Pulsars ......................... 3

1.1.1 Classifications of Pulsars . . . . . . . . . . . . . . 4

1.1.2 Pulsar Emission . . . . . . . . . . . . . . . . 8

1.1.2.1 Pulse Profile Evolution . . . . . . . . . . . . . . 10

1.1.2.2 Pulsar Spectral Index . . . . . . . . . . . . . . 13

1.1.3 Pulsar Timing . . . . . . . . . . . . . . . . . . 14

1.2 The Interstellar Medium . . . . . . . . . . . . . . . . . . . . . . . . . 19

1.2.1 Dispersion . . . . . . . . . . . . . . . . . . 19

1.2.2 Scattering and Scintillation ............... . . . 22

1.3 Gravitational Waves . . . . . . . . . . . . . . . . 26

1.3.1 Gravitational Wave Sources . . . . . . . . . . . . . . 27

1.3.2 Pulsar Timing Arrays . . . . . . . . . . . . . . . . . 30

1.4 Observing Pulsars . . . . . . . . . . . . . . . . . . . . . . . . . . . . . . . . 34

1.4.1 Signal Propagation Path . . . . . . . . . . . . . 35

1.4.2 Signal Simulation . . . . . . . . . . . . . . . 37

1.5 Thesis Outline . . . . . . . . . . . . . . . . . 38

2 Radio Properties of Rotating Radio Transients 40

2.1 Introduction . . . . . . . . . . . . . . . . . . . . . 40

2.2 Data . . . . . . . . . . . . . . . . . . . 44

2.3 Methods . . . . . . . . . . . . . . . . . . 46

2.3.1 Single-pulse Spectral Index Analysis . . . . . . . . . . . . . . 46

2.3.1.1 Identification of Single pulses . . . . . . . . . . . 46

2.3.1.2 Fitting Single-pulse Templates . . . . . . . . . . . . 48

2.3.1.3 Obtaining a Single-pulse Spectral Index . . . . . . . 51

2.3.2 Analysis of Simulated Data . . . . . . . . . . . . . . . 55

2.3.3 RRATs Analyzed in This Work . . . . . . . . . . . . . . 57

2.3.4 Wait-time Analysis . . . . . . . . . . . . . . 63

2.3.5 Pulse Energy Analysis . . . . . . . . . . . . . . . 65

2.4 Results and Discussion . . . . . . . . . . . . . . . . 66

2.4 .1 Spectral Indices . . . . . . . . . . . . . . . . 66

2.4 Wait Times . . . . . . . . . . . . . . . . 70

2.4.2.1 Wait-time Distribution Analysis . . . . . . . . . 70

2.4.2.2 Flux-density-Wait-time Correlation . . . . . . . . . . 80

2.4.3 Energy Distributions . . . . . . . . . . . . . . . . . 80

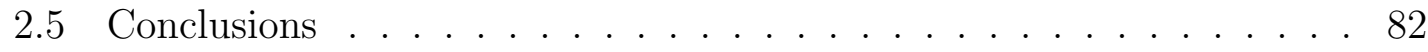


3 Characterizing the Noise In Millisecond Pulsars 85

3.1 Introduction . . . . . . . . . . . . . . . . . . 85

3.2 Observations . . . . . . . . . . . . . . . . . . . . . 90

3.2.1 Observational Data . . . . . . . . . . . . . . 90

3.2.2 Data Reduction . . . . . . . . . . . . . . . . . 91

3.3 Methods . . . . . . . . . . . . . . . . . 94

3.3.1 White Noise in Pulsar Timing Residuals . . . . . . . . . . . . 94

3.3.2 Scintillation Parameters and $\sigma_{\text {DISS }}$. . . . . . . . . . . . . . . . 98

3.3.2.1 Measuring Scintillation Parameters . . . . . . . . . . 99

3.3.3 Secondary Spectra . . . . . . . . . . . . . . . . . . 104

3.3.4 Maximum Likelihood Estimates of Pulse Jitter . . . . . . . . . 105

3.3.5 Pulse Jitter Sub-Band Correlations . . . . . . . . . . . . . . 106

3.3.6 Measuring DM Variations on Hour Timescales . . . . . . . . . 107

3.3.7 Timing Precision with Non-Contiguous TOAs . . . . . . . . . 110

3.4 Scintillation Parameters and Secondary Spectra . . . . . . . . . . . 111

3.4.1 Scintillation Parameters Results and Discussion . . . . . . . . 112

3.4.1.1 Scintillation Bandwidths . . . . . . . . . . . 115

3.4.1.2 Scintillation Timescales . . . . . . . . . . . . 116

3.4.1.3 Uniform Media vs Thin Screens . . . . . . . . . . . 117

3.4.1.4 Scintle Drift Rates . . . . . . . . . . . . . . . . 118

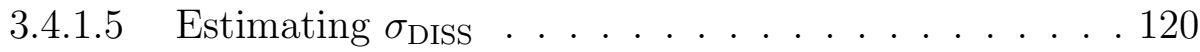

3.4.2 Secondary Spectra Results and Discussion . . . . . . . . . . 121

3.5 Pulse Jitter Results and Discussion . . . . . . . . . . . . . . . . . 121

3.5.1 Pulse Jitter Meausured from $\sigma_{\mathcal{R}}$. . . . . . . . . . . . . . . . . 122

3.5.2 Maximum Likelihood Jitter Results . . . . . . . . . . . . . . . 124

3.5.3 Sub-band Correlation Results . . . . . . . . . . . . . . . 126

3.6 DM Variations on Short Timescales . . . . . . . . . . . . . . . . . 127

3.7 Timing Precision of Non-contiguous Timing Residuals Results and

Discussion . . . . . . . . . . . . . . . . . . . 132

3.8 Conclusions . . . . . . . . . . . . . . . . . . . . . . . . . 133

4 Measuring Noise in Pulsar Timing Through Simulations 136

4.1 Introduction . . . . . . . . . . . . . . . . . . 136

4.2 PsrSigSim Description . . . . . . . . . . . . . . . . . . . . 139

4.3 Methods . . . . . . . . . . . . . . . . . . . . . 142

4.3.1 Generating Simulated Data . . . . . . . . . . . . . 143

4.3.2 TOAs and Residuals . . . . . . . . . . . . . . . . . . 151

4.4 Simulated Data . . . . . . . . . . . . . . . . . . . 153

4.4.1 Frequency-Independent Pulse Profile Simulations . . . . . . . 156

4.4.2 Frequency-Dependent Pulse Profile Simulations . . . . . . . . 162

4.5 Results . . . . . . . . . . . . . . . . . . . . 167

4.5.1 Discussion of Frequency-Independent Profile Simulations . . . 169

4.5.2 Discussion of Frequency-Dependent Profile Simulations . . . . 177

4.6 Implications for Precision Pulsar Timing . . . . . . . . . . . . . . . . 180

4.7 Conclusions . . . . . . . . . . . . . . . . . . . . . . 184 
5 Conclusion 186

5.1 Understanding the Properties of RRATs . . . . . . . . . . . . . . 186

5.2 Importance of Quantifying Noise in PTAs and Characterizing the ISM 188

5.3 Utilizing Simulations for Evaluating PTA Noise Budgets . . . . . . . 190

5.4 Looking Ahead . . . . . . . . . . . . . . . . . . . . 191

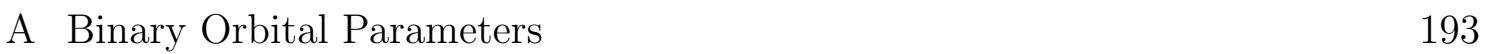




\section{List of Tables}

2.1 RRAT parameters . . . . . . . . . . . . . . 53

2.2 RRAT scattering parameters . . . . . . . . . . . . . . . 61

2.3 RRAT mean spectral indices . . . . . . . . . . . . . . . . 67

2.4 RRAT wait-time model statistics $\ldots \ldots \ldots \ldots \ldots$

2.5 RRAT flux density model statistics . . . . . . . . . . . . . 79

3.1 MSP Pulsar Parameters . . . . . . . . . . . . . . . . . . . . . 92

3.2 MSP Fit Scintillation Parameters . . . . . . . . . . . . . . . . . . . . . . . . . .

3.3 MSP Scintillation-derived Parameters . . . . . . . . . . . . . . . . 114

3.4 Estimated MSP pulse jitter . . . . . . . . . . . . . . . . . . . 122

3.5 Estimates of MSP timing error . . . . . . . . . . . . . . . 125

4.1 Simulated pulsar parameters . . . . . . . . . . . . . . . . 154

4.2 Simulated backend/receiver parameters . . . . . . . . . . . . 157

4.3 Simulated pulsar DM variation parameters . . . . . . . . . . 158

4.4 Description of simulated data sets . . . . . . . . . . . . . 159

4.5 PSR J1744-1134 no profile evolution simulation fitting results . . . . 170

4.6 PSR J1744-1134 profile evolution simulation fitting results . . . . . . 171

4.7 PSR B1855+09 no profile evolution simulation fitting results . . . . . 172

4.8 PSR B1855+09 profile evolution simulation fitting results . . . . . . . 173

4.9 PSR B1953+29 no profile evolution simulation fitting results . . . . . 174

4.10 PSR B1953+29 no profile evolution simulation fitting results . . . . 175 


\section{List of Figures}

$1.1 \quad P-\dot{P}$ Diagram $\ldots \ldots \ldots \ldots \ldots \ldots \ldots \ldots \ldots$

1.2 Pulsar Pulse Trains . . . . . . . . . . . . . . . . . . . . 7

1.3 Simple Dipole Pulsar Model . . . . . . . . . . . . . . . . . . . . 11

1.4 Pulse Profile Evolution with Radio Frequency . . . . . . . . . . . . 12

1.5 Pulse Jitter . . . . . . . . . . . . . . . . . . . . . . . . . . . . . . . 15

1.6 Timing Residuals Example . . . . . . . . . . . . . . . . . . 18

1.7 Dispersed Pulse . . . . . . . . . . . . . . . . . . . 21

1.8 Scintillation \& Scattering Geometry . . . . . . . . . . . . . . . . . 23

1.9 Pulse Scatter Broadening Example . . . . . . . . . . . . . . . . . 24

1.10 Gravitational Wave Spectrum . . . . . . . . . . . . . . . . . . 29

1.11 Hellings-Downs Curve . . . . . . . . . . . . . . . . . . 33

2.1 Single-pulse baseline variation subtraction comparison . . . . . . . . 48

2.2 Spectral index distribution for simulated RRAT data . . . . . . . . 54

2.3 Sample of single-pulses from PSR 1819-1458 . . . . . . . . . . 58

2.4 Sample of single-pulse spectral index fits from PSR J1819-1458 . . . 59

2.5 Single-pulse wait-time distribution for PSR J1819-1458 - Parkes . . . 60

2.6 RRAT single-pulse spectral index distributions . . . . . . . . . . . 64

2.7 Single-pulse wait-time distribution for PSR J1819-1458 - GBT . . . 71

2.8 Single-pulse wait-time distribution for PSR J1913+1330 . . . . . . . . 72

2.9 Single-pulse wait-time distribution for PSR J1317-5759 . . . . . . . . 73

2.10 RRAT single-pulse flux versus wait-time distributions . . . . . . . 76

2.11 RRAT single-pulse flux density distributions . . . . . . . . . . . . 77

3.1 Ustretched MSP dynamic spectra . . . . . . . . . . . . . . . 100

3.2 PSR J1832-0836 dynamic spectrum autocorrelation comparison . . . 103

3.3 MSP timing residual $\mathrm{rms}$ error versus integration time . . . . . . . 123

3.4 MSP DM variations on short timescales . . . . . . . . . . . . . . 128

3.5 MSP timing residual rms error for non-contiguous timing residuals . . 131

4.1 Simulated pulse profile comparison for PSR B1855+09. . . . . . . 146

4.2 DMX values for non-frequency dependent profile simulations . . . . . 161

4.3 Simulation FD differences for PSR J1744-1134 . . . . . . . . . . 162

4.4 Simulation FD differences for PSR B1855+09 . . . . . . . . . . . 163

4.5 Simulation FD differences for PSR B1953+29 . . . . . . . . . . . 164

4.6 DMX values for frequency dependent profile simulations . . . . . . 165

4.7 DMX versus injected scattering timescale for PSR J1744-1134 . . . . 179

4.8 DMX versus injected scattering timescale for PSR B1855+09 . . . . 180

4.9 DMX versus injected scattering timescale for PSR B1953+29 . . . . 181 


\section{Chapter 1}

\section{Introduction}

Neutron stars (NSs), the cores of dead stars with initial masses greater than $\sim 8 M_{\odot}$, are some of the most interesting objects in the Universe. NSs are extraordinarily dense, with masses of $\sim 1.2-2 M_{\odot}$ and radii of just $\sim 10 \mathrm{~km}$. A sub-population of NSs are detectable as pulsars, extremely precise, rapidly rotating NSs that emit radio waves like a lighthouse (Hewish et al., 1968). As the pulsar rotates, the radio waves are detectable as short bursts, or pulses, of emission as the beam moves across our line-of-sight (LOS) on Earth. There are many classes of pulsars, some emitting pulses regularly and some more sporadically (McLaughlin et al., 2006), spanning a range of spin periods, from $1.39 \mathrm{~ms}$ (Hessels et al., 2006) to 23.5 seconds (Tan et al., 2018).

The emission from pulsars spans the electromagnetic spectrum, but the radio emission specifically is influenced by different effects depending on the frequency. These effects range from varying the intensity of the emission (Lorimer et al., 1995) to the delays in the pulse arrival time at Earth due to dispersion or scattering from the ISM (Rickett, 1977). While these effects are quantifiable, they all introduce some measure of noise in precision pulsar timing. The characterization and mitigation of this noise and these effects become important for the most precise astrophysics that can be done with pulsars. 
One particularly interesting astrophysical experiment that pulsars can be used for is the detection of gravitational waves $(\mathrm{GWs})$, which are ripples in spacetime that are predicted as a consequence of Einstein's field equations within general relativity. The first direct detection of GWs was made by the Laser Interferometer Gravitational-Wave Observatory (LIGO) in 2015 from inspiraling stellar mass black holes at frequencies of tens to hundreds of hertz (Abbott et al., 2016). GWs can be emitted by many other sources as well. Pulsar timing arrays (PTAs), for example, are sensitive to nanohertz GWs emitted by orbiting supermassive black hole binaries (SMBHBs) at the centers of galaxy mergers. But the detection of GWs at these frequencies require extremely precise $\sim$ sub-microseconds timing precision for all pulsars in the PTA (Lam et al., 2019). For these experiments, the characterization and mitigation of noise is critical for detection (Lam et al., 2016a), similar to the characterization and mitigation in other GW detectors, such as LIGO.

In this chapter we will review the various classes of pulsars and their emission properties as well as the basics of pulsar timing. We will discuss the frequencydependent effects on pulsar emission as the pulses travel through the ISM, and how these effects add noise to PTAs in the context of nanohertz GW detection. Finally, we discuss how these effects may be characterized and how the covariances between them may be untangled through simulations of pulsar timing data. 


\subsection{Pulsars}

Since their discovery by then-graduate student Jocelyn Bell Burnell (Hewish et al., 1968), pulsars have been at the forefront of some of the most important astronomical and astrophysical discoveries. The first exoplanets ever detected were found orbiting a pulsar by Wolszczan \& Frail (1992). The first binary pulsar system was discovered by Hulse \& Taylor (1975), and this discovery garnered them a Nobel Prize in 1993 as it was found that the orbital separation of the system was shrinking due to energy loss from GWs exactly as predicted by general relativity (GR). This was the first indirect observation of GW emission. Pulsars in double and triple systems have since been used for some of the most stringent tests of GR (e.g. Kramer et al., 2006; Archibald et al., 2018).

As NSs are the second densest objects in the Universe, behind only black holes, studying their equation of state is also of great interest. Cromartie et al. (2020) recently measured the mass of PSR J0740+6620 at $2.14_{-0.09}^{+0.10} M_{\odot}$ at the $68.3 \%$ confidence interval, leading to the most constraining limits to date on the neutron star equation of state. Millisecond pulsars (MSPs) are currently being used to constrain the nanohertz GW background from supermassive black hole binaries (e.g. Arzoumanian et al., 2018a, 2020a,b). The field of pulsar astronomy is still rich and continues to make great astrophysical discoveries. 


\subsubsection{Classifications of Pulsars}

At the time of writing, there are 2,872 known pulsars according to the ATNF Pulsar Catalog${ }^{1}$ (Manchester et al., 2005). These pulsars fall into a few different categories based on a number of different characteristics. Most of the categories can be seen visually when plotting the spin period against the spin period derivative for the known population, often referred to a $P-\dot{P}$ diagram, as in Figure 1.1. The main category of pulsars we will refer to as "canonical" pulsars. These are pulsars with $P \gtrsim 30 \mathrm{~ms}$ and $\dot{P}$ around $10^{-15} \mathrm{~s} \mathrm{~s}^{-1}$ that emit a pulse every time they rotate and their beam of radio emission passes across our LOS. Using the $P$ and $\dot{P}$, one can also estimate a pulsar's magnetic field strength, $B$, and its characteristic age, $\tau_{C}$. Assuming a pulsar with a radius of $10 \mathrm{~km}$, a moment of intertia of $10^{45} \mathrm{~g} \mathrm{~cm}^{2}$, and a $90^{\circ}$ angle between the pulsar's spin and magnetic axes, the magnetic field can be roughly computed as

$$
B=3.2 \times 10^{19} \mathrm{G} \sqrt{P \dot{P}} .
$$

This leads to $B$ of around $10^{12} \mathrm{G}$, for canonical pulsars. Similarly, if one assumes that $P$ is much larger than it was when the pulsar was born and that the pulsar's spin-down is due completely to magnetic dipole radiation, then its age can be approximated as

$$
\tau_{C}=\frac{P}{2 \dot{P}}
$$

This estimate, or characteristic age, is usually a good estimate for canonical pulsars to within an order of magnitude. Most known pulsars, including the first pulsars

\footnotetext{
${ }^{1}$ https://www.atnf.csiro.au/research/pulsar/psrcat
} 
every discovered (Hewish et al., 1968), fall into this category of canonical pulsars. They are generally grouped into the upper right cluster of pulsars in the $P-\dot{P}$ diagram shown in Figure 1.1.

The second main category of pulsars are millisecond pulsars, or MSPs as previously mentioned. The first of these MSPs, PSR B1937 + 21, was discovered by Backer et al. (1982) and has a spin period of $1.558 \mathrm{~ms}$. The general definition for a MSP is a pulsar with $P \lesssim 30 \mathrm{~ms}, \dot{P}$ around $10^{-20} \mathrm{~s} \mathrm{~s}^{-1}$, and $B$ around $10^{8} \mathrm{G}$, with almost 400 known at the time of this writing (Manchester et al., 2005). The much shorter spin periods of MSPs come about due to a process commonly referred to as "recycling", where a pulsar accretes material and angular momentum from its binary companion and as a result has a decrease in its spin period. Since this process typically occurs when the pulsar's companion is in a red giant phase, we expect most MSPs to be in binary systems with white dwarf companions (e.g. Bhattacharya \& van den Heuvel, 1991; Freire \& Tauris, 2014). This process of "spinning up" the MSP typically leads to narrower pulse widths due to the smaller spin periods that it imparts. Additionally, the recycling processes significantly decreases the MSP's magnetic field, and hence spin-down rate, which makes the timing of MSPs more stable over long timespans than their canonical counterparts. The necessarily narrow pulse widths observed for MSPs subsequently allow for more precise measurements of when the pulses arrive at the telescope compared to canonical pulsars. This category of pulsars can be seen grouped together in the lower left corner of Figure 1.1.

We can further break down these two groups into a number of different groups, 


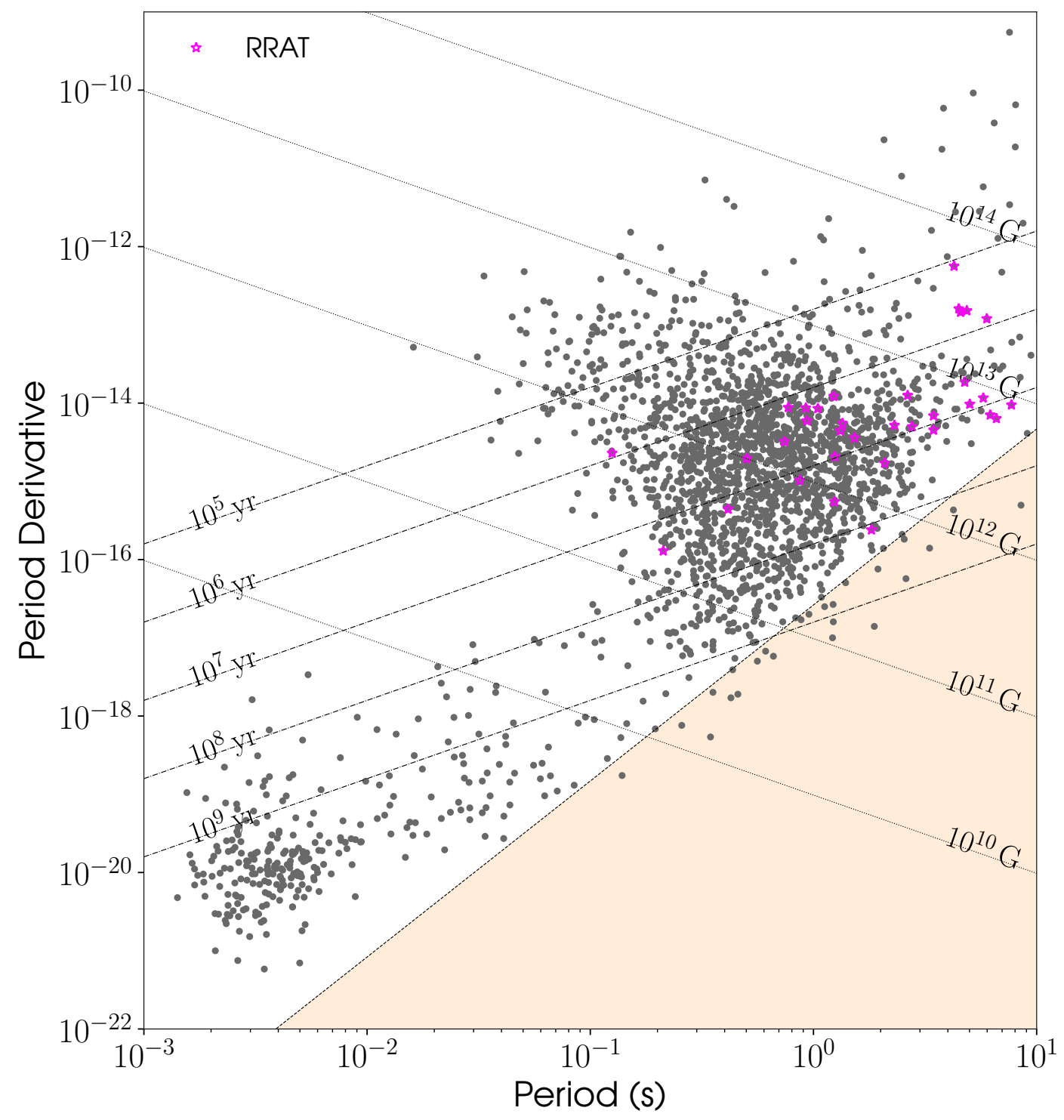

Figure 1.1 Diagram showing the pulsar population as a function of period and period derivative. In addition, lines of constant magnetic field as computed by Equation 1.1 and constant age as computed by Equation 1.2 are shown. The orange region in the lower right represents the pulsar "graveyard" as computed by Eq. 4 of Zhang et al. (2000). This region shows where the pulsars' $P$ and $\dot{P}$ are such that the potential drop between the open and closed magnetic field lines is too small for pair production to occur, so the neutron star is longer observable as a radio pulsar. MSPs can be seen in the cluster in the lower left corner, and RRATs are highlighted in purple. This figure was generated using data from the ATNF Pulsar catalog (Manchester et al., 2005) and the psrqpy software package (Pitkin, 2018). 

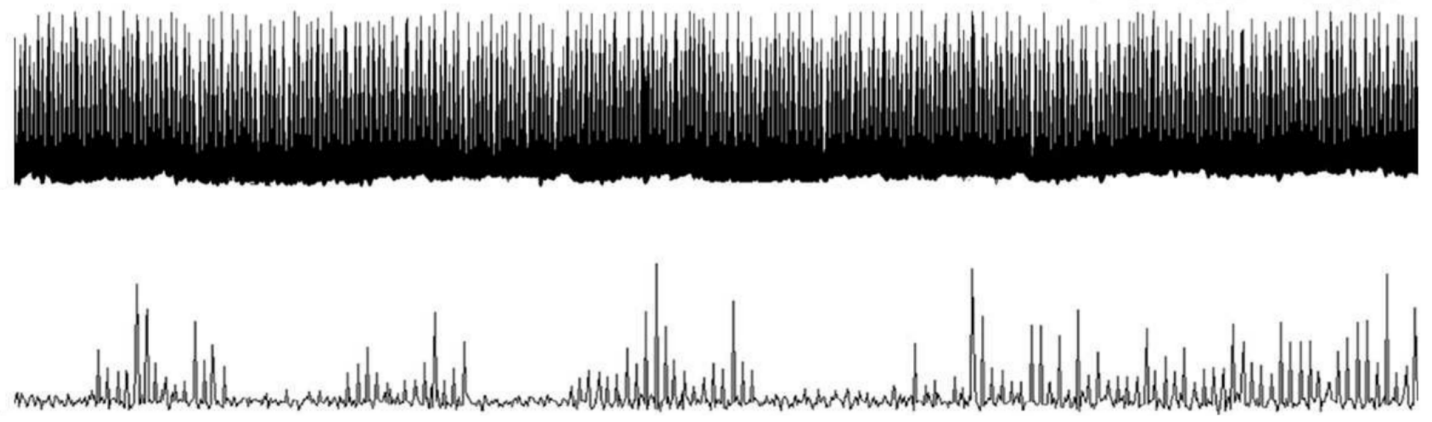

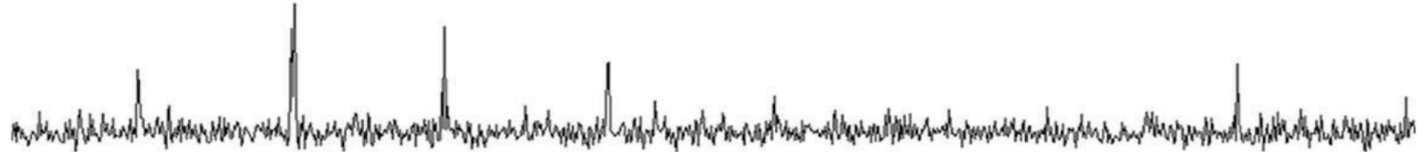

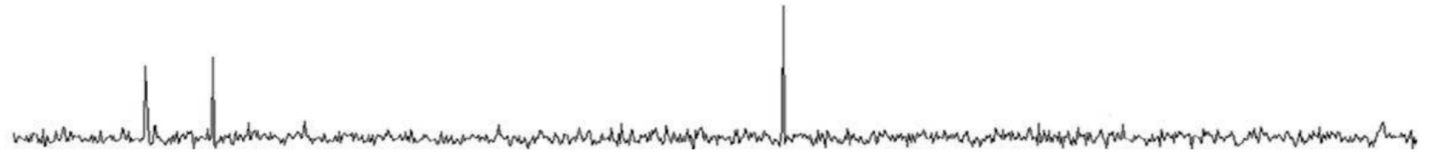

Figure 1.2 Top: Single pulses emitted by the Vela pulsar showing consistant pulsed emission. Upper Middle: Pulses from a nulling pulsar, PSR J1646-6831, showing consistent but periodic emission. Bottom Middle \& Bottom: Pulses from two RRATs, PSRs J1647-36 and J1226-32, showing periodic, but sporadic, emission. Figure adapted and reproduced with permission from Burke-Spolaor (2013), permission conveyed through Copyright Clearance Center, Inc. 
such as nulling pulsars, where the emission is suddenly undetectable for time spans ranging from seconds to hours, first seen by Backer (1970). An example of this on-and-off type of emission is shown in the second panel from the top in Figure 1.2. However, as nulling pulsars still have phases where every pulse they emit is detectable, they are still typically discovered through the standard pulsar periodicity Fourier domain searches. A more extreme phenomena are rotating radio transients, or RRATs, first discovered by McLaughlin et al. (2006). These are pulsars that emit sporadic, but periodic, pulses such that one can only discover them by searching for single pulses. The sporadic nature of the pulse emission from RRATs compared to nulling and standard pulsars is shown in Figure 1.2. This population of RRATs are also marked in Figure 1.1, and have spin-down properties similar to those of canonical pulsars.

\subsubsection{Pulsar Emission}

While pulsars have been found to emit at many different wavelengths, my work focuses on the emission and observation of pulsars purely in the radio regime. The answer to what the exact physical processes are behind pulsar radio emission is still unknown, though it is a field of active research. However, a basic emission model can be understood as a rotating magnetic dipole (Goldreich \& Julian, 1969).

Simply, a rotating magnetic field will induce an electric field, and as NSs have a very large magnetic field and rotate very quickly, a very strong electric field will be induced at the surface of the pulsar. This electric field then creates a force on 
charged particles, primarily electrons, stronger than the gravitational force of the NS. These charged particles are then pulled off of the surface of the NS, travel along its magnetic field lines, and create a plasma that surrounds it, also known as the magnetosphere of the NS. This plasma rotates with the NS up until the rotation speed of the plasma reaches the speed of light. At these rotation speeds, the dipole magnetic field lines that the particles are accelerated over may not be closed and are referred to as open field lines along the magnetic poles of the NS.

As these particles travel along the magnetic field lines, they emit curvature radiation, which produces high-energy photons that interact with low-energy photons through the magnetic field, producing electron-positron pairs. These pairs then create more high-energy photons through curvature radiation in a cascading effect effect that create groups of charged particles. These groups accelerate along the open magnetic field lines due to the large drop in electric potential between the open and closed lines caused by the extreme rotational speeds of the pulsar. The exact mechanism for achieving a coherent beam is still a field of active research. Methods for achieving this coherence in these simplistic models are discussed in detail in Ginzburg \& Zhelezniakov (1975), though new models of pulsar emission are proposed regularly (e.g. Philippov et al., 2019).

As the pulsar continues to rotate, these beams of coherent emission rotate along with it, creating the lighthouse emission effect seen as these beams cross the LOS of a radio telescope, classifying the NS as a pulsar. As the pulsar rotates, each time this beam of emission passes across our radio telescope it appears as a characteristic pulse of emission. We can measure the time of arrival (TOA) of this 
pulse extremely accurately at the telescope. The highly stable rotational period of a pulsar, and our ability to fit for other effects, allows us to then create a model of when they will arrive so that we can accurately predict the arrival time of future pulses at the telescope.

\subsubsection{Pulse Profile Evolution}

It is also known that the widths and shapes of pulsar pulse profiles vary depending on the radio frequency at which they are emitted (e.g. Cordes, 1978; Pennucci et al., 2014; Pennucci, 2019). This intrinsic pulse profile evolution with radio frequency comes from the height of the radio emission in the pulsar magnetosphere, with higher frequency radio emission originating from closer to the pulsar's surface. As the cone of emission narrows closer to the pulsar's surface, as depicted in Figure 1.3, the width of the pulse profile's components will also narrow. This profile evolution is generally less pronounced in MSPs than in canonical pulsars (Kramer et al., 1999; Chen \& Wang, 2014; Dai et al., 2015; Lam et al., 2016a), though across large frequency ranges it may still be large for MSPs, as shown in Figure 1.4.

The difference in emission heights also explains why some profiles appear to have different shapes depending on the observed radio frequency, as shown in Figure 1.4. Since different radio frequencies can be mapped to the height of the emission (Cordes, 1978), they show different parts of the pulsar's beam of emission. Each

individual component of the pulsar's profile is then different at an observed radio frequency, so the composite profile of the pulsar may change dramatically. This 


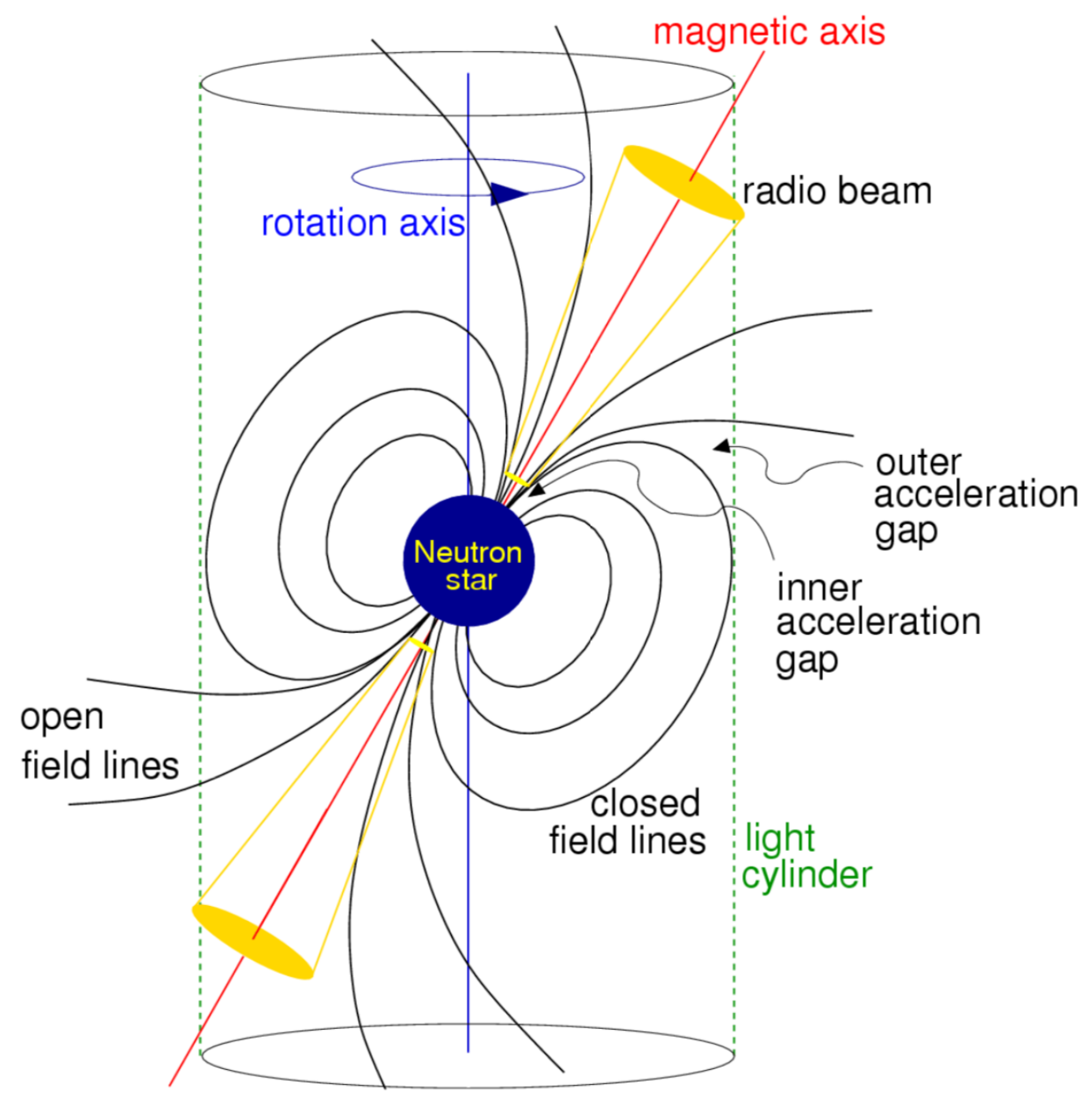

Figure 1.3 Simplified cartoon model of magnetic dipole pulsar emission. The central neutron star is shown with its rotation axis in blue offset from the magnetic axis in red. Both the open and closed magnetic field lines are shown, with the beams of coherent radio emission shown by the yellow cones. The light cylinder marks the boundary where plasma surrounding the pulsar co-rotates with the pulsar at the speed of light, and hence denotes the boundary of the magnetosphere. The inner and outer acceleration gaps show where the plasma density exceeds the maximum density allowed by the simple magnetic dipole model discussed in $\S 1.1 .2$. Here the plasma is depleted creating a so-called gap in the pulsar magnetosphere. This image comes from the Handbook of Pulsar Astronomy (ISBN: 9780521828239, Lorimer \& Kramer, 2004) and has been reproduced with permission of Cambridge University Press through PLSclear. 


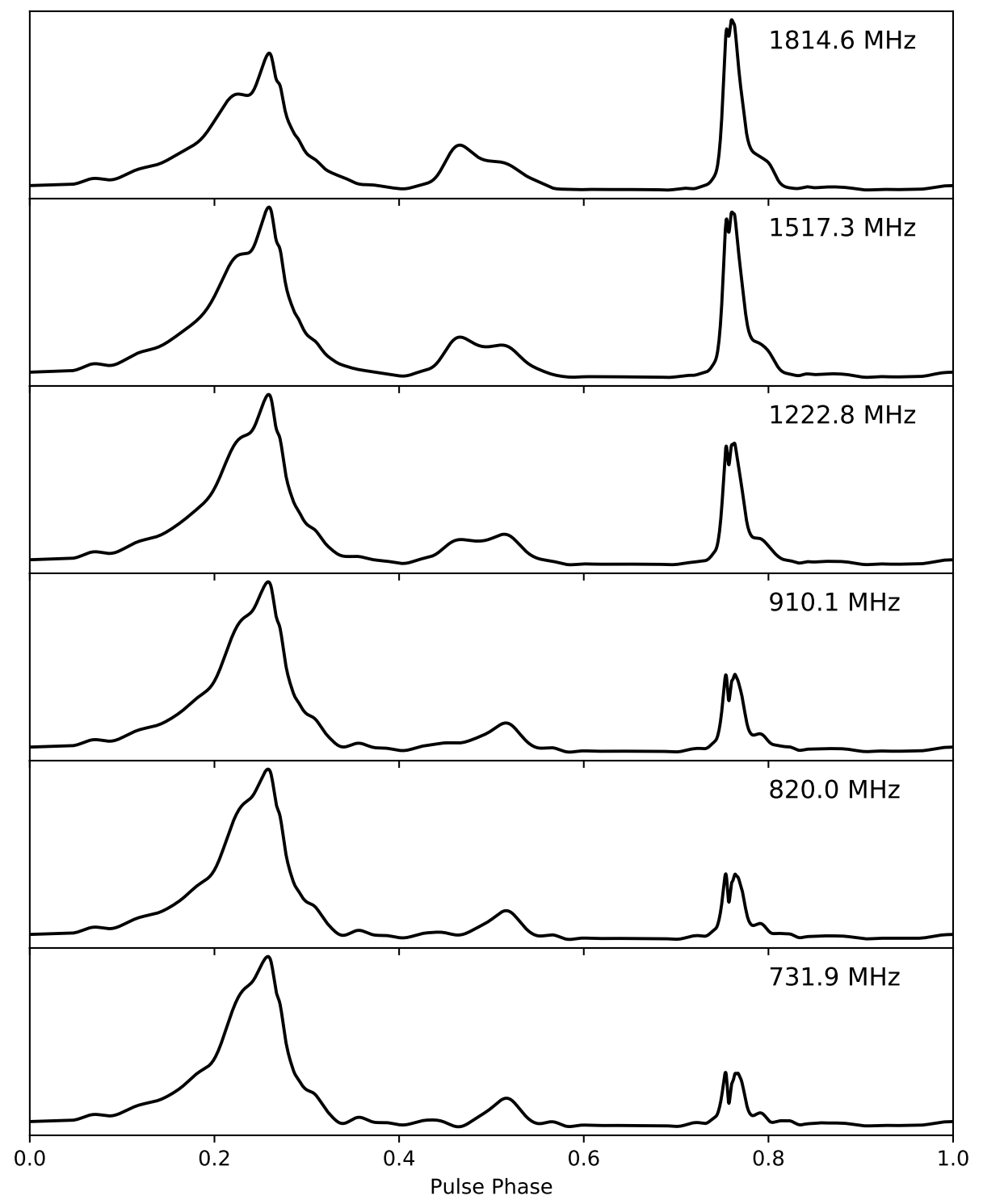

Figure 1.4 Example of pulse profile evolution with radio frequency seen in PSR J0931-1902. Across different radio frequencies, the components of the pulse profile clearly show shape changes. These profile models were created from wideband timing pulse profile templates released as part of the NANOGrav 12.5-yr data release (Alam et al., 2020a). 
model explains pulse profile features either appearing or disappearing at different observed radio frequencies (Rankin, 1983), or apparently shifting their location in the pulse profile (Gangadhara \& Gupta, 2001).

\subsubsection{Pulsar Spectral Index}

Pulsars are generally considered to be weak radio sources with fluxes that are often two or three orders of magnitude smaller than "bright" radio sources like active galactic nuclei (e.g. Jankowski et al., 2018). However they emit across a large span of radio frequencies, or what is called broadband emission. In fact, pulsars have been observed from frequencies as low as $10 \mathrm{MHz}$ (Stovall et al., 2015) to as high as $101 \mathrm{GHz}$ (Liu et al., 2019). However, a pulsar's flux density, $S$, here the integrated intensity of a pulse profile, is known to scale as a power law with observing frequency, $\nu$, at least to a first approximation, such that

$$
S_{\nu} \propto \nu^{\alpha}
$$

where $\alpha$ is known as the spectral index of the pulsar.

Large scale studies of pulsar spectral indices such as Jankowski et al. (2018) have found that on average pulsars have fairly steep spectral indices, following a roughly log-normal distribution with an average of $\alpha=-1.60 \pm 0.03$. These studies have also found that some pulsars do not follow a simple power law as in Eq. 1.3 and instead are best fit with broken power law or spectral turnover models, which could be due to thermal free-free absorption (Rajwade et al., 2016), synchrotron 
self-absorption, or absorption of the emitted radio photons by an associated pulsar wind nebula (Jankowski et al., 2018). The steep spectral index of most pulsars means that their fluxes will be much greater at lower radio frequencies and so one may expect most pulsar observations for all kinds of experiments to be done at these lower frequencies. However, a number of effects due to the ISM, discussed in $§ 1.2$, make the optimal observing frequencies for pulsars vary depending on the experiment being done.

\subsubsection{Pulsar Timing}

For many studies, the quantity of most interest for observations of pulsars is the time of arrival (TOA) of the radio pulses at the telescope. However, as discussed above, pulsars are generally very weak radio sources, and so the individual (or single) pulses are often too weak to be observed. In order to obtain a high signal-to-noise $(\mathrm{S} / \mathrm{N})$ ratio we often utilize the $P$ and $\dot{P}$ to coherently sum many single pulses together, a process which is often referred to as folding, to create the pulse profile. Folded pulse profiles are typically very stable, though it may require hundreds to thousands of summed pulses to produce a stable folded profile (Rathnasree \& Rankin, 1995). However, the shapes and phases of individual pulses may vary, an intrinsic effect known as pulse jitter, shown in Figure 1.5, which will also add unmodeled, time-variable structure to the profile (Shannon \& Cordes, 2010; Lam et al., 2019).

Nominally the TOA of a pulse is referenced to some fiducial point on the pulse 

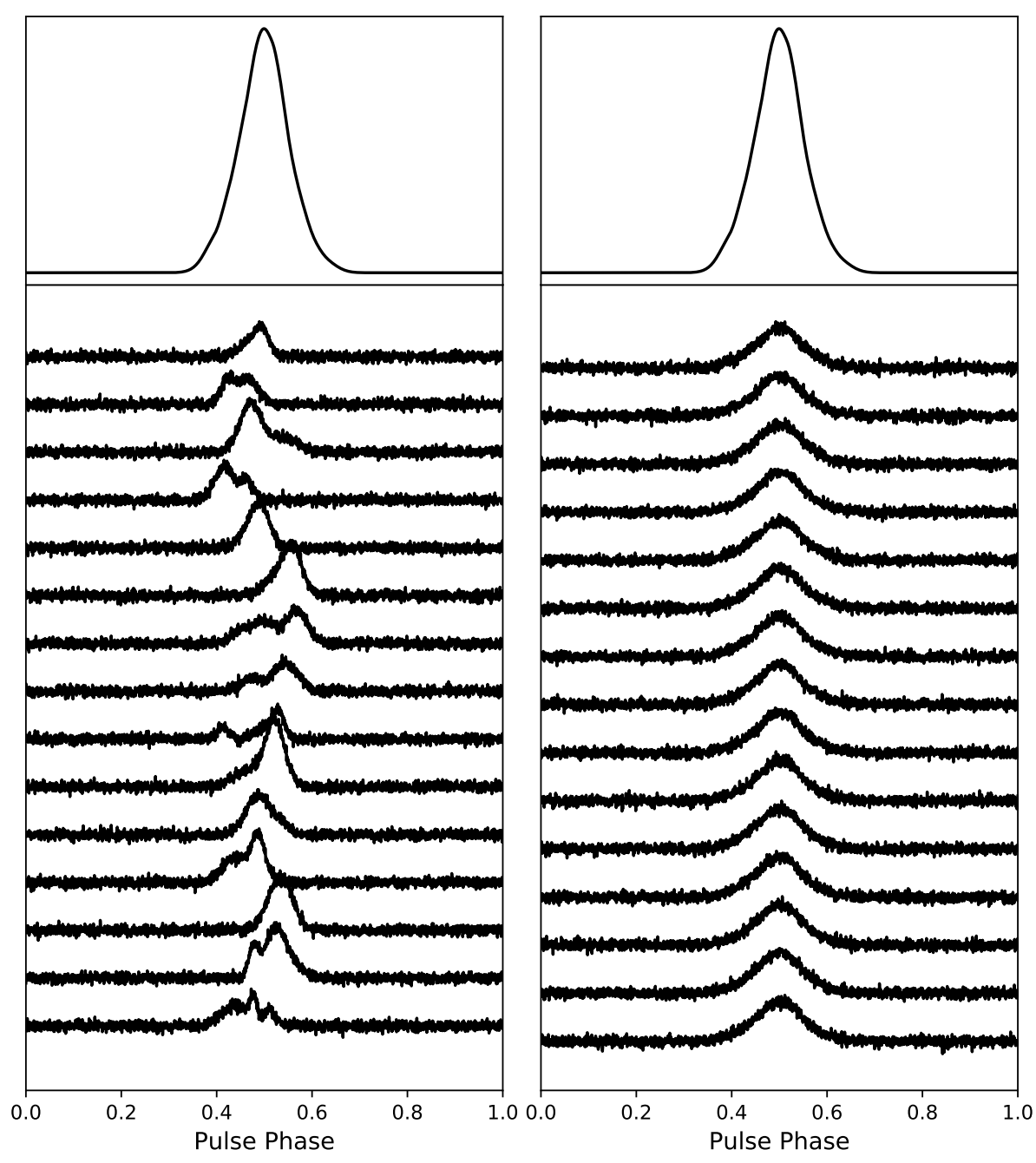

Figure 1.5 Simulated example of pulse jitter. The left plot shows pulse-to-pulse shape variations, or jitter, while the right plot shows constant pulse profile shapes over time. The sum of either sets of pulses produces the same composite pulse profile, show in the top panels. 
profile. The measurement of pulse TOAs is done by cross-correlating a high $\mathrm{S} / \mathrm{N}$ template of the pulse profile with the observed, usually folded, profile, typically using a $\chi^{2}$ minimization method in the frequency domain. This method assumes that the observed pulse profile, $P$, at a given time $t$ is a scaled and shifted version of the template profile, $T$, with some additional noise, $N$, such that

$$
P(t)=a+b \times T(t-\tau)+N(t) .
$$

Here $a$ is an arbitrary shift, $b$ is a scaling factor, and $\tau$ is the time shift between the fiducial point of the template and the profile referenced to the start time of the observation, yielding the TOA of the profile. While the profiles are discretely sampled in time at the telescope, if this cross-correlation is done in Fourier space the TOA precision will equal roughly the ratio of the width to the $\mathrm{S} / \mathrm{N}$ of the observed profile (Taylor, 1992).

The process of modeling and predicting the pulse TOAs and quantifying any variations between the model predictions and actual TOAs is called pulsar timing. The simplest version of a pulsar timing model predicting the pulse phase, $\phi$, at a given time $t$ is a Taylor expansion around the pulsar spin frequency, $f$, referenced to some fiducial phase $\phi_{0}$ at time $t_{0}$, which gives

$$
\phi(t)=\phi_{0}+f\left(t-t_{0}\right)+\frac{1}{2} \dot{f}\left(t-t_{0}\right)^{2}+\cdots
$$

Many additional delays and effects may need to be added to this timing model to 
accurately predict $\phi(t)$. These include, but are not limited to, the orbital motion of pulsars in binary systems, astrometric effects, and effects from the ISM, which must all be considered when creating a pulsar timing model. In addition, since our observing frame is non-inertial, pulse TOAs are referenced to the Solar System barycenter, or center of mass, before a timing model is produced.

An accurate timing model is created by minimizing the differences between the observed pulse TOAs and the timing model predictions. These differences are referred to as timing residuals. This process is usually done using some type of least-squares minimization to the timing model parameters with software such as TEMPO $^{2}$ (Nice et al., 2015) or PINT ${ }^{3}$ (Luo et al., 2020). The errors on the fit parameters in a timing model are then obtained from the resulting covariance matrix. Any unmodeled parameters, e.g. parameters that should be included but are not, will manifest as structures or patterns in the pulsar's timing residuals that indicate that these parameters must be added. Examples of some of these types of structures are shown in Figure 1.6. Not all of these structures or patterns are visible by-eye, however, so statistical methods such as a two-sided F-test (Snedecor \& Cochran, 1989) may be employed. In the context of pulsar timing, the F-test compares the significances of various additional parameters being added or removed in a pulsar timing model. The F-test is the current method used by the NANOGrav PTA to determine whether a pulsar's existing timing model needs to have parameters added or removed (Arzoumanian et al., 2016).

\footnotetext{
${ }^{2}$ http://tempo.sourceforge.net/

${ }^{3}$ https://github.com/nanograv/PINT
} 

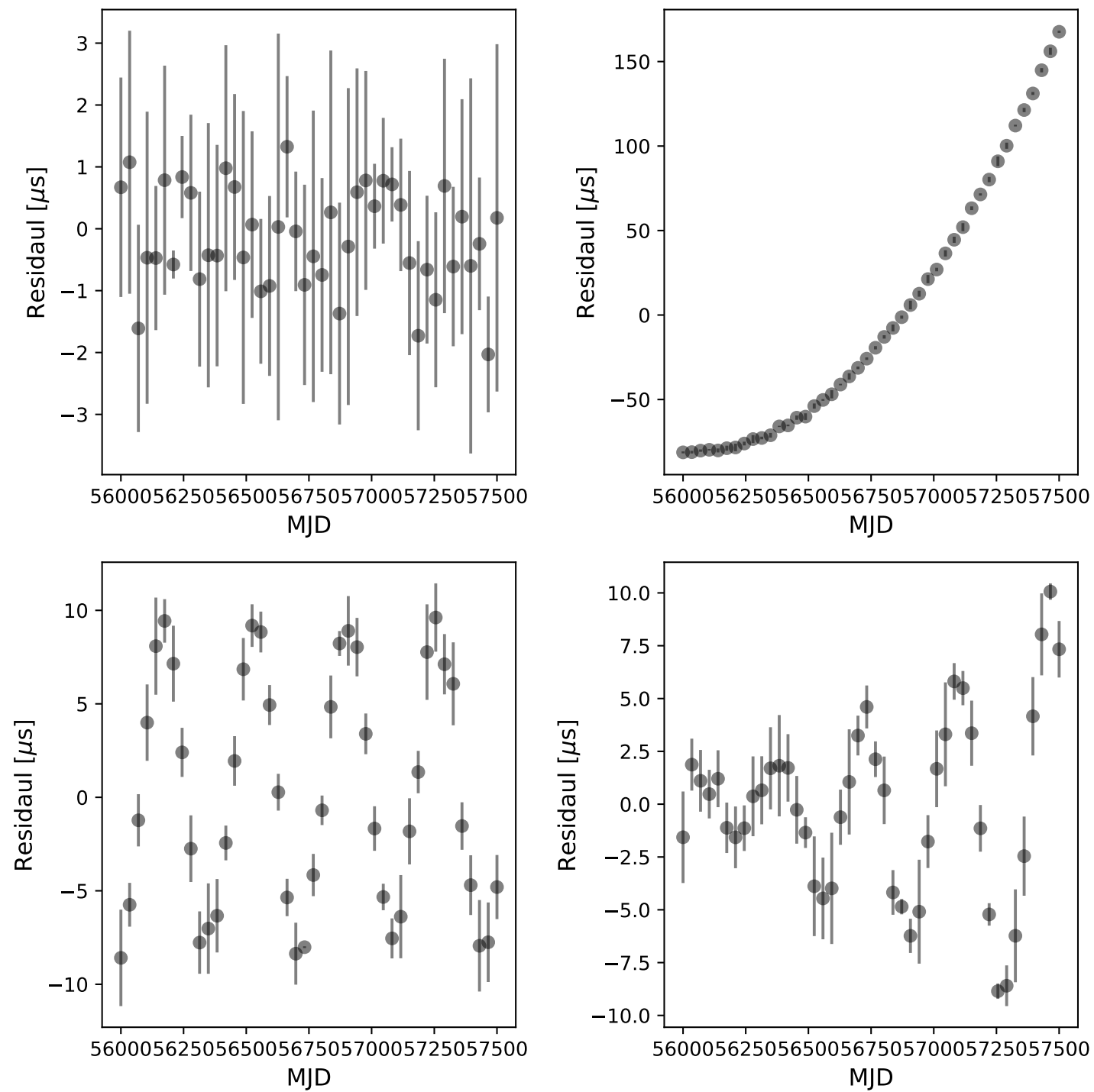

Figure 1.6 MSP timing residuals simulated with the PINT pulsar timing software with random white noise added (Luo et al., 2020). Upper Left: Timing residuals with the correct timing model. All residuals are scattered around zero, showing that the timing model is accurately measuring all expected effects and predicting the pulse TOAs accurately. Lower Left: Slightly incorrect pulsar position creates a yearly trend in the residuals. Upper Right: Slightly underestimated spindown rate results in an exponential trend in timing residuals. Lower Right: Slightly incorrect proper motion results in a steadily increasing yearly sinusoidal pattern in the timing residuals. 


\subsection{The Interstellar Medium}

As radio emission from pulsars travels through interstellar space it passes through the ISM, which is made up of (primarily hydrogen) gas and dust. In particular, as the radio waves pass through ionized plasma in the ISM they are delayed and scattered by some amount dependent on the frequency of the radio emission.

\subsubsection{Dispersion}

The free electrons in the ISM will cause the radio pulse to be dispersed, such that lower radio frequencies of the broadband pulsed emission arrive later than the higher frequencies, as shown in Figure 1.7. In addition to the radio frequency of the emission, the scale of this dispersive delay is dependent on the integrated column density of free electrons along the LOS to the pulsar, also known as the dispersion measure (DM),

$$
\mathrm{DM}=\int_{0}^{d} n_{e}(l) d l
$$

Here $d$ is the distance to the pulsar and $n_{e}$ is free electron density along the LOS, $l$. Since DM depends on the distance to the pulsar, it is possible obtain a rough estimate of a pulsar's distance based of off its DM using models of the electron density of the Milky Way such as those by Cordes \& Lazio (2002) and Yao et al. (2017). In the Milky Way, we find that $<n_{e}>\approx 0.03 \mathrm{~cm}^{-3}$ (e.g. Ables \& Manchester, 1976) leading to a general rule that a DM of $30 \mathrm{pc} \mathrm{cm}^{-3}$ means the pulsar is roughly 1 kpc away from Earth.

The dispersive delay that is observed is proportional to the radio frequency as 
$\nu^{-2}$, and the delay between two frequencies can be calculated as

$$
\Delta t_{\mathrm{DM}} \simeq 4.15 \times 10^{6} \mathrm{~ms} \times \mathrm{DM}\left(\frac{1}{\nu_{1}^{2}}-\frac{1}{\nu_{2}^{2}}\right)
$$

Here $\Delta t_{\mathrm{DM}}$ is the total time delay in ms between the two frequencies $\nu_{1}$ and $\nu_{2}$, in MHz. DM is defined as in Eq 1.6 and is in units of $\mathrm{pc} \mathrm{cm}^{-3}$. From Eq. 1.7 it is easy to see that the delay will be larger at lower observing frequencies, though $\Delta t_{\mathrm{DM}}$ will depend on the spacing between $\nu_{1}$ and $\nu_{2}$. For pulsar timing experiments, it is critical to correct for this delay between the high and low observing frequencies. This is highlighted in Figure 1.7 which shows that for PSR B1855+09, which has a DM of $13.30 \mathrm{pc} \mathrm{cm}^{-3}$, the delay across one observing band from 980 to $1780 \mathrm{MHz}$ is $40.05 \mathrm{~ms}$, which is much larger than its period of $5.362 \mathrm{~ms}$ (Manchester et al., 2005).

The DM will also vary slightly with time. This is due not only to the turbulence and movement of the ISM, but also to the pulsar moving across the sky, which may move its LOS close to the Sun, where the solar wind may change the observed DM (e.g. Madison et al., 2019). While these changes, even over timescales of years, are usually relatively small, $\lesssim 10^{-2} \mathrm{pc} \mathrm{cm}^{-3}$, for precision timing experiments it is critical to account for these epoch-to-epoch variations. Measurements of a pulsar's DM and its variations over time also allow us to characterize the ISM itself, as in Jones et al. (2017), and can be used to study variations in the ISM on small scales of just a few AU (Lam et al., 2018b). Methods for characterizing the ISM along the LOS to the pulsar, as well as physical properties of the pulsar that can be derived 


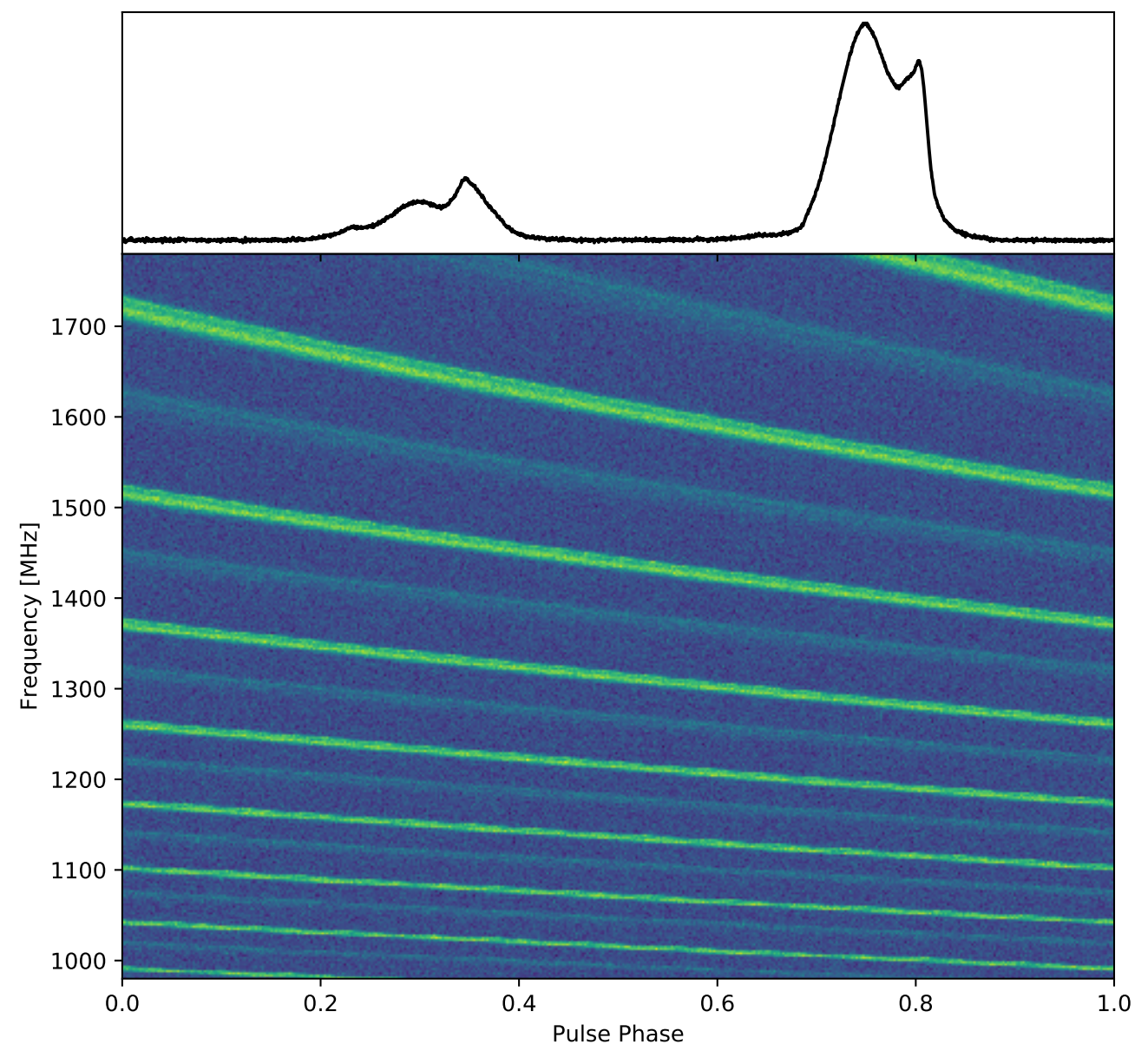

Figure 1.7 Example of a dispersed pulse using simulated data from PSR B1855+09. Top: The dispersion-corrected pulse profile of PSR B1855+09 at $1380 \mathrm{MHz}$ integrated in time and frequency. The profile template comes from the NANOGrav 11-year data release (Arzoumanian et al., 2016). Bottom: Dispersed pulse shown across $800 \mathrm{MHz}$ of bandwidth clearly showing the $\nu^{-2}$ dispersion relation. The dispersive delay is larger than the pulse period resulting in the pulse phase wrapping across this large bandwidth. 
from these characterizations, such as its transverse velocity, are discussed in greater detail in Chapter 3.

\subsubsection{Scattering and Scintillation}

In addition to dispersive delays, radio pulses from pulsars are also scattered due to inhomogeneities in the ISM. As shown in Figure 1.8, as the radio waves propagate they will be slightly scattered and some will subsequently arrive later than others resulting in a broadening, or "scatter-broadened" pulse profile. The scale of pulse scattering is also frequency dependent. While there are many models of pulse scattering, the simplest one approximates the ISM as a turbulent Kolmogorov medium (Kolmogorov, 1941) in a single thin screen halfway between the pulsar and the Earth (Scheuer, 1968). In this model, the pulse scattering delays scale as $\nu^{-4.4}$, and are thus larger at lower observing frequencies, though there are a wide range of measured frequency scaling indices ranging from $\sim-1$ to -6 (e.g. Keith et al., 2013; Levin et al., 2016; Turner et al., 2020).

An example of this scatter broadening at multiple frequencies is shown in Figure 1.9. One way the effects of scatter broadening are modeled is by an exponential function $e^{-t / \tau_{\mathrm{d}}}$, where $\tau_{\mathrm{d}}$ is the scattering timescale, with the intrinsic pulse profile, described by discrete time bins, $t$. Because $\tau_{\mathrm{d}}$ varies with time and depends on the radio frequency (e.g. Levin et al., 2016; Turner et al., 2020), this deconvolution is usually done for each observation (e.g. Kirsten et al., 2019; Dolch et al., 2020).

While the added time delays due to pulse scattering are usually quite small, 


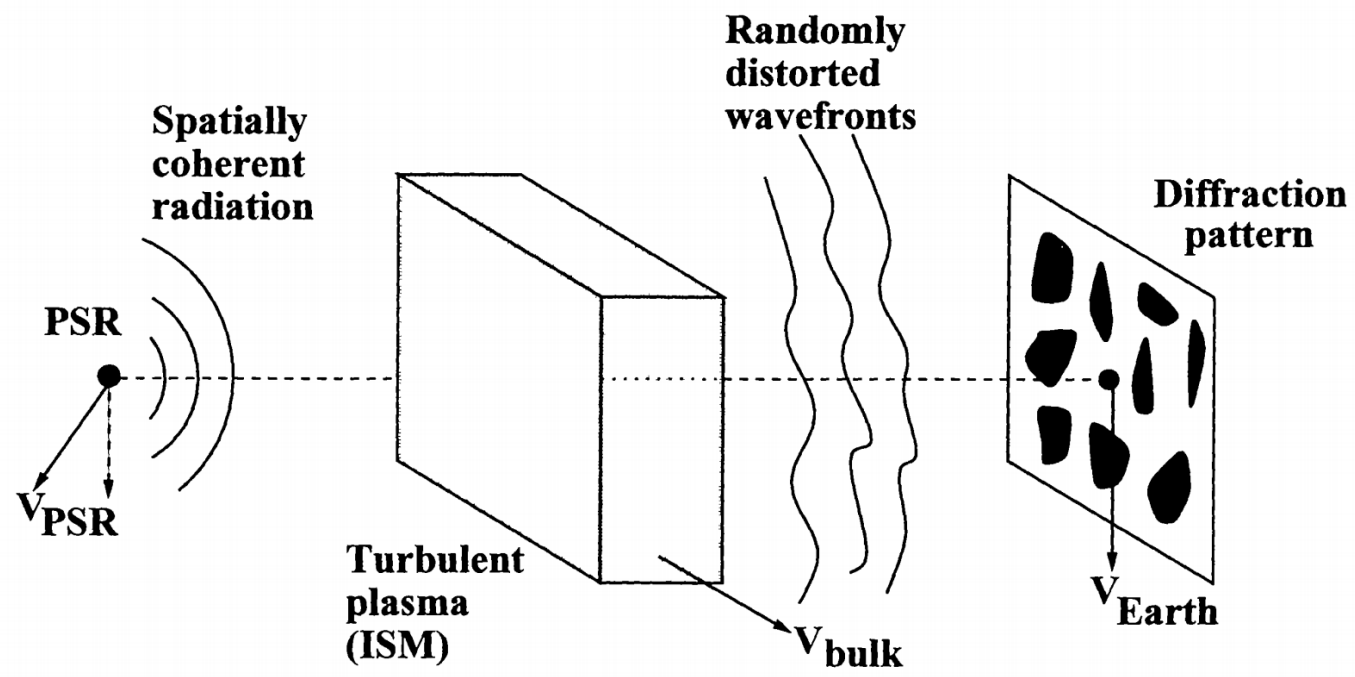

Figure 1.8 Path of propagation for pulsar radio emission showing pulse scattering by the turbulent ISM, resulting in a diffraction pattern, or scintillation screen, at the Earth. Figure reproduced with permission from Cordes (2002).

since they are frequency dependent this scattering is covarient with the DM. This can cause measurements of the DM to partially absorb the effects of pulse scattering, resulting in incorrect measurements of the DM and thus characterization of the ISM. This covariance is discussed and explored in greater detail in Chapter 4.

In addition to scatter broadening and delays, the inhomogeneities in the ISM also cause the scattered radio waves to interfere with each other both constructively and destructively. This creates an effect called scintillation, essentially an interference pattern on the observing plane where the pulse may be brighter at some radio frequencies and times than at others. An example of this interference is shown in Figure 1.8, and it creates a pattern of bright spots, or scintles. The scintles for each pulsar are described by a characteristic timescale, $\Delta t_{\mathrm{d}}$, and bandwidth, $\Delta \nu_{\mathrm{d}}$, depending on the LOS to the pulsar and its velocity. To measure $\Delta t_{\mathrm{d}}$ and $\Delta \nu_{\mathrm{d}}$, one may measure the intensity of the pulsar emission in the frequency-time plane, also 


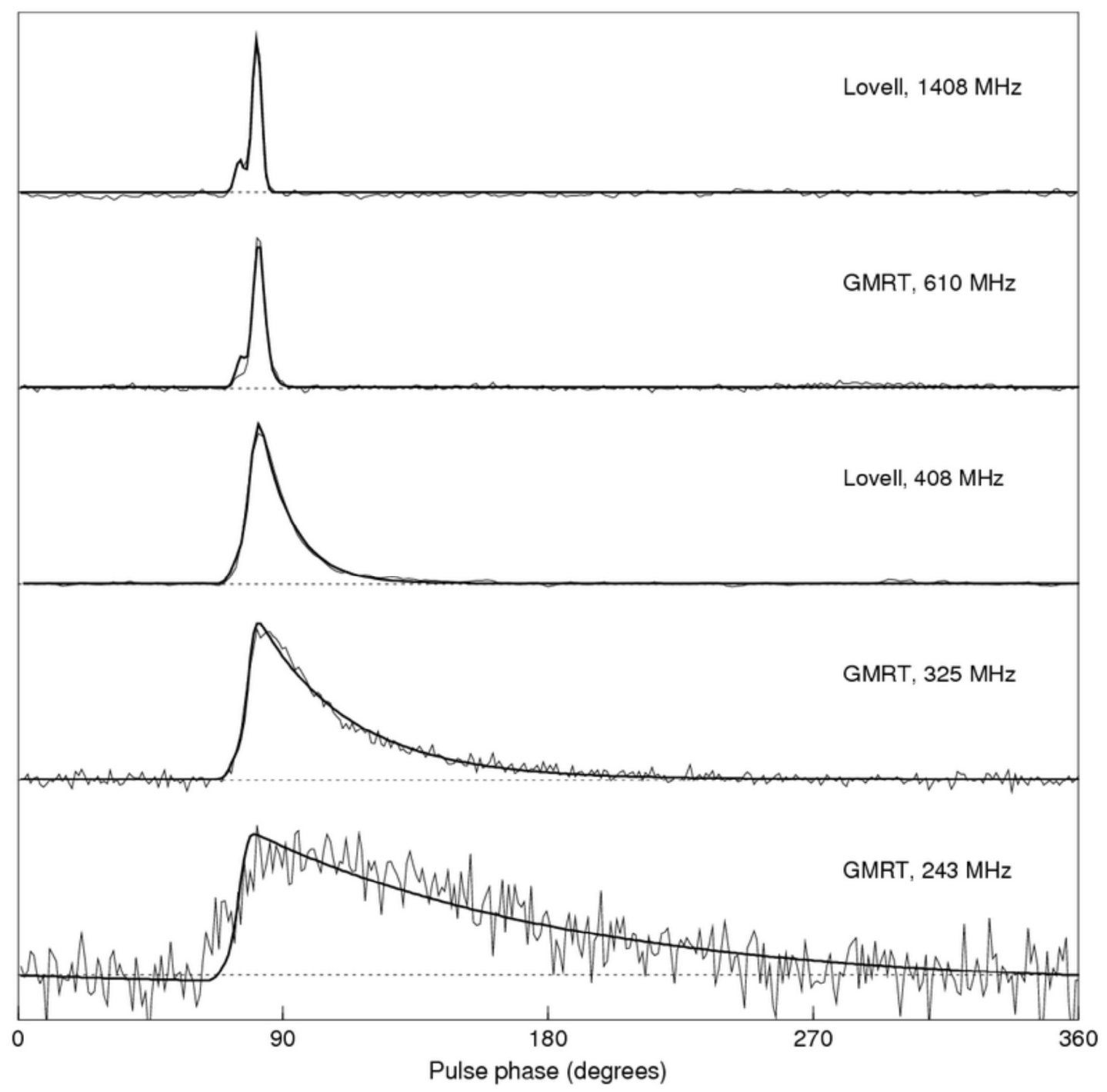

Figure 1.9 Example of pulse scatter broadening as a function of observing radio frequency for PSR B1831-03. As described in $\$ 1.2 .2$ it is clear that the effects of pulse scatter broadening are larger at lower frequencies. This image comes from the Handbook of Pulsar Astronomy (ISBN: 9780521828239, Lorimer \& Kramer, 2004). and has been reproduced with permission of Cambridge University Press through PLSclear. 
known as a dynamic spectrum. Depending on the total bandwidth and length of the observation, as well as the frequency and time resolution, one may or may not observe scintillation and be able to make these measurements. The methods used to analyze a dynamic spectrum are described in more detail in Chapter 3.

If one can measure $\Delta \nu_{\mathrm{d}}$ from the dynamic spectrum, than $\tau_{\mathrm{d}}$ can be obtained as

$$
\tau_{\mathrm{d}}=\frac{C_{1}}{2 \pi \Delta \nu_{\mathrm{d}}}
$$

where $C_{1}$ is a coefficient ranging from $0.6-1.5$ depending on the geometry and spectrum of the electron density of the ISM (Lambert \& Rickett, 1999). This method of measuring the scatter broadening timescale from the dynamic spectrum is typically used more often than the deconvolution method described above. Typical pulsar observation lengths are $<\Delta t_{\mathrm{d}}$, however, if $\Delta t_{\mathrm{d}}$ can also be measured, then one can obtain an estimate of the pulsar's transverse velocity, $V_{\text {ISS }}$. The relationship between $\Delta \nu_{\mathrm{d}}, \Delta t_{\mathrm{d}}$, and $V_{\text {ISS }}$ is described by Cordes \& Rickett (1998),

$$
V_{\mathrm{ISS}}=A_{\mathrm{ISS}} \frac{\sqrt{d \Delta \nu_{\mathrm{d}}}}{\nu \Delta t_{\mathrm{d}}}
$$

where $A_{\text {ISS }}$ is a constant that depends on $C_{1}$ and assumptions on whether the ISM is uniform or not, and $d$ is the distance between the Earth and the pulsar. As shown by Cordes \& Rickett (1998), assuming the ISM is well described by a uniform Kolmogorov medium such that $C_{1}=1.16$ (Lambert \& Rickett, 1999), one will find $A_{\text {ISS }}=2.53 \times 10^{4} \mathrm{~km} \mathrm{~s}^{-1}$. For some pulsars, measurements of the scintillation pa- 
rameters may be the only way to constrain their transverse velocities if their proper motions cannot be measured. If the scintillation parameters can be measured precisely enough though, then the transverse velocity of the pulsar may be constrained even more accurately than from precision pulsar timing (Reardon, 2018; Reardon et al., 2019).

\subsection{Gravitational Waves}

Gravitational waves (GWs) were first predicted to exist as a consequence of general relativity (GR; Einstein, 1915). Instead of a force between two objects, as in the Newtonian description of gravity, GR describes gravity as the influence an object's mass has on four-dimensional spacetime curvature. As objects that have mass move, they change the curvature of spacetime, with more massive objects causing the most dramatic changes. With this definition of gravity, GWs are like ripples in spacetime. GWs are caused by non-spherically symmetric changes in an object's, or system's, gravitational quadrupole moment, q, which describes the mass distribution of the object or system, and may be computed as (Jones, 2018)

$$
\mathbf{q}=\sum_{i j}\left(m_{i}+m_{j}\right) \mathbf{x}_{i} \mathbf{x}_{j}
$$

Here $m$ is the mass of an object in the system and $\mathbf{x}$ is the object's three dimensional position vector.

As an example, consider a binary system, such as a pair of black holes, $i$ and j. As they orbit, their positions, $\mathbf{x}_{i}$ and $\mathbf{x}_{j}$, will change, which may change $\mathbf{q}$ of the 
system, so this binary pair will emit GWs. As these GWs propagate outward, they carry energy away from the system and perturb spacetime as they propagate at the speed of light.

As the GW travels it squeezes and stretches space. This perturbation is often characterized by a strain, $h$, which describes a fractional change in the distance between two objects. For two objects with some mass a distance $L$ apart, we measure

$$
h=\frac{\delta L}{L}
$$

where $\delta L$ is the change in $L$ caused by the GW. The values of $\delta L$ from GWs are

$\ll L$, meaning that any experiment attempting to detect GWs must be able to measure both $L$ and $\delta L$ extremely precisely. They must also be able to quantify all other sources of noise in their detectors, as some of these noise processes may manifest in ways that could be falsely identified as signals from a GW.

\subsubsection{Gravitational Wave Sources}

There are many different sources of GWs, including different types of binary systems, such as merging stellar mass black hole systems or binary neutron stars in compact orbits. In fact, GWs from both of these types of sources have been observed by the Laser Interferometer Gravitational-Wave Observatory (LIGO). The first ever detection of GWs was the stellar mass black hole binary merger GW151226, which was observed between 35 and $450 \mathrm{~Hz}$ (Abbott et al., 2016). Similarly, GWs from a double neutron star merger, GW170817, was also detected by LIGO between 24 
and $500 \mathrm{~Hz}$ (Abbott et al., 2017a). This double neutron star merger also produced a kilonova which was observed across the electromagnetic spectrum (e.g. Abbott et al., 2017b). At the time of writing 50 total GW events have been detected by LIGO. Merging sources such as these make up the only directly detected gravitational wave events so far, and are shown on the right-hand of Figure 1.10 in purple.

However, other sources of GWs will emit at different frequency ranges, as shown in Figure 1.10. The limits to the GW sensitivity of a PTA at the upper bound in the GW frequency band are due to the cadence of pulsar observations, and at the lower bound they are limited by the total length of time over which the pulsar has been observed. With these limits in mind, we will focus on sources of GWs that emit at nanohertz frequencies.

The primary contributors to nanohertz GWs in the Universe are likely supermassive black hole binaries (SMBHBs) found at the centers of galaxies that have merged. Evidence for an SMBH in the Milky Way, often referred to as Sagittarius A*, or Sag A*, was found by Ghez et al. (1998) and it is now believed that all galaxies have a SMBH at their center (e.g. Kormendy \& Richstone, 1995; Kormendy \& Ho, 2013). When galaxies merge the SMBHs at their centers will follow the gravitational potential well of the newly merged galaxy, and fall towards its center, eventually forming a binary. After forming a binary, the SMBHB will evolve to smaller separations over time. The processes through which this evolution occurs are not fully understood, though it is likely a combination of dynamical friction and other effects (Burke-Spolaor et al., 2019). When the binary separation of the SMBHB reaches $\lesssim 0.1 \mathrm{pc}$, it will start to emit nanohertz GWs which may be detectable by PTAs. 


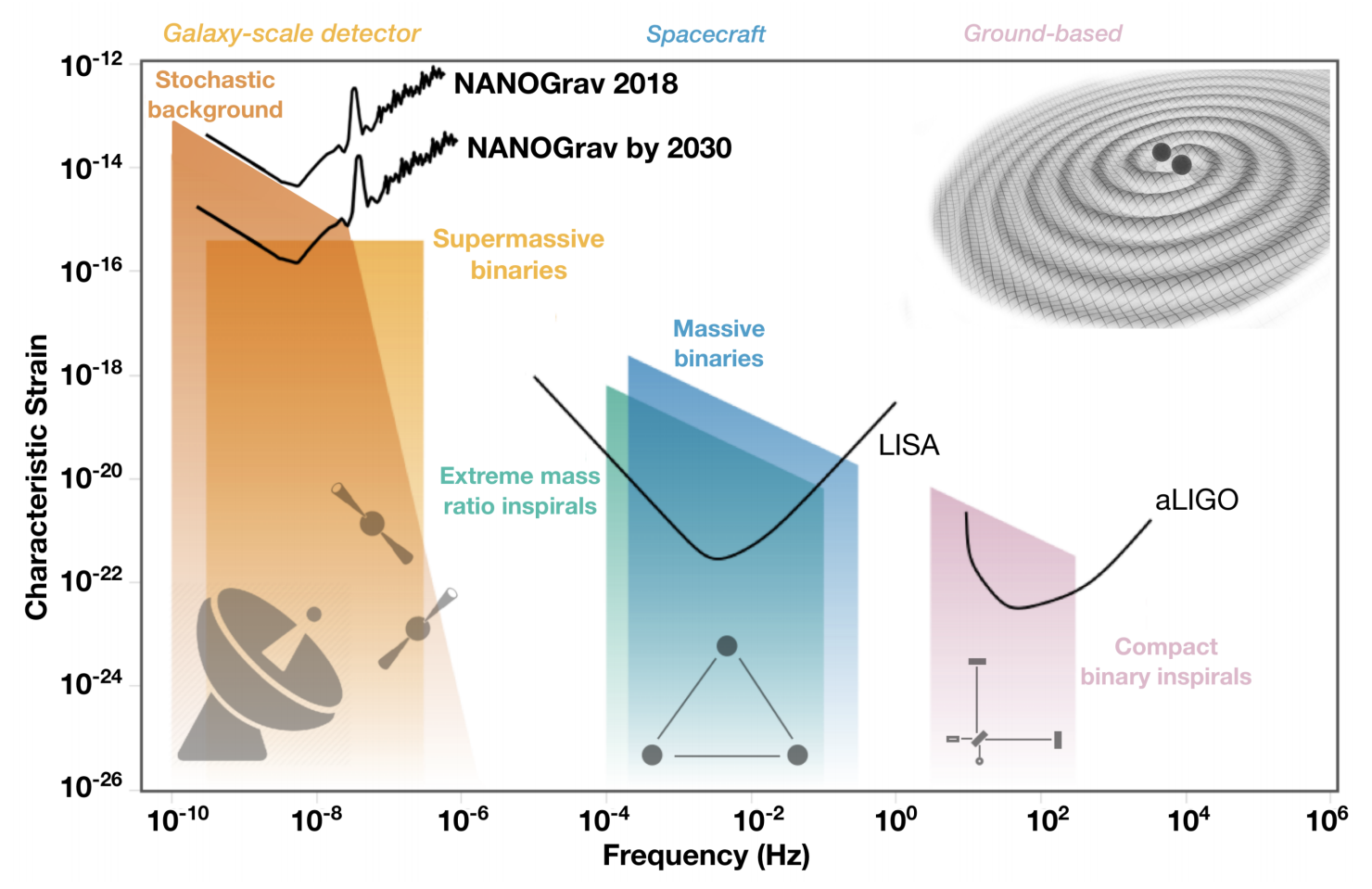

Figure 1.10 Example GW spectrum showing different sources of GWs across a large range of frequencies and strains. The solid black lines also show the estimated sensitivity curves for different GW detectors. Image Credit S. Taylor \& C. Mingarelli, adapted from gwplotter.org (Moore et al., 2015) and based on a figure in Mingarelli \& Mingarelli (2018). Illustration of merging black holes adapted from R. Hurt/Caltech-JPL/EPA. This figure is reproduced with permission from Ransom et al. (2019) under the Creative Commons Attribution 4.0 International License (CC-BY 4.0). 
It is also possible that cosmic strings, theorized fundamental filaments of energy formed in the early Universe that stretch to cosmological scales, may produce nanohertz GWs (e.g. Vachaspati \& Vilenkin, 1985; Leblond et al., 2009; Dror et al., 2020). This may occur when the strings form loops with each other which then break off and oscillate, producing the GWs. In addition to cosmic strings, it has been theorized that some types of dark matter may also emit nanohertz GWs (e.g. Aldabergenov et al., 2021; Kitajima et al., 2021).

The sum of all the GW signatures from these sources collectively make up what is called the stochastic GW background (SGWB), a nanohertz GW signal that is likely stronger than any individual source, and therefore the likely first detectable nanohertz GW signal. The detection of the SGWB would help inform on the number of SMBHBs in the Universe and how they evolve, which in turn would yield information about galaxy merger rates and SMBH formation.

\subsubsection{Pulsar Timing Arrays}

Due to the long periods of nanohertz GW sources, which may span years to decades, it is impossible to build a ground-based detector, as the arm lengths would need to span many lightyears. This is the basis for utilizing a PTA as a GW detector as pulsars span distances of up to a few kpc from Earth. As a GW propagates through space, it may expand or contract the proper distance between Earth and the pulsar causing the radio pulses to arrive slightly earlier or later than expected. These delays are very small, on the order of $\lesssim 10$ s of ns, but since pulsars, 
particularly MSPs, can be timed very precisely, these delays may be apparent in the pulsar timing residuals. However, pulsars have intrinsic noise like pulse jitter, and extrinsic effects, due to the ISM for example, which may cause delays in the TOAs of the pulses that could be confused for a GW signal in the individual pulsar. Because of this, one must use an array of pulsars spread across the sky and search for correlations in pulse delay times between pairs of MSPs.

The effects of a GW signal $s_{+, \times}$at the pulsar (the "pulsar term") will differ from its effects at the Earth (the "Earth term") due to the large distances between Earth the pulsars. The difference between the GW signal, $\Delta s_{+, x}$, at the pulsar and the GW signal at Earth may be written as (e.g. Arzoumanian et al., 2020a)

$$
\Delta s_{+, \times}(t)=s_{+, \times}\left(t_{p}\right)-s_{+, \times}(t)
$$

Where $t$ is the time at which the GW passes the solar system barycenter (SSB), and $t_{p}$ is the time at which it passes the pulsar. These times are related to each other geometrically as

$$
t_{p}=t-L(1+\hat{\Omega} \cdot \hat{p})
$$

Where $L$ is the distance to the pulsar, $\hat{\Omega}$ is a unit vector pointing between the pulsar and the SSB, and $\hat{p}$ is a unit vector pointing from Earth to the pulsar. If the pulsar term can be observed, it will characterize an earlier time in the GW signal's evolution than the signal we observe at the SSB. A full description of the GW signal model can be found in Arzoumanian et al. (2020a) and references therein. 
Regardless of whether the GW source is a single SMBHB or is due to the SGWB, the expected correlation between any given pair of MSPs is based on their angular separation on the sky. We expect the SGWB to be stronger than any individual source of nanohertz GWs, and as it is the sum of all sources, its signal will be largely independent of direction. The key to searching for an SGWB signal in a PTA is to look for Earth term correlations in the timing residuals of all pulsars in the array. We do not look for correlations in the pulsar term as the pulsars are all at different distances from the Earth, and from each other, and these distances are not constrained to within a GW wavelength, so we cannot detect correlations in the pulsar term (e.g. Arzoumanian et al., 2020a). This correlation for this background was worked out by Hellings \& Downs (1983) as a set of coefficients

$$
\alpha_{i j}=\frac{\left(1-\cos \gamma_{i j}\right)}{2} \ln \left(\frac{\left(1-\cos \gamma_{i j}\right)}{2}\right)-\frac{1}{6} \frac{\left(1-\cos \gamma_{i j}\right)}{2}+\frac{1}{3}
$$

Here $\gamma_{i j}$ is the angle between two pulsars $i$ and $j$ in the array. The quadrupolar nature of GW emission results in the residuals of MSPs in either the same or opposite directions on the sky being correlated, while those that are separated by roughly $90^{\circ}$ will be anti-correlated. This theoretical curve showing the correlations across the full range of angular separations, called the Hellings-Downs Curve, is shown in Figure 1.11. Detecting these Hellings-Downs correlations is the crucial mark of the detection of the SGWB. As a real PTA will have a finite number of MSPs, there will be a finite number of discrete angles defining pairs of pulsars in the array that will be used to fill out this curve. So to make a detection of the SGWB, it 


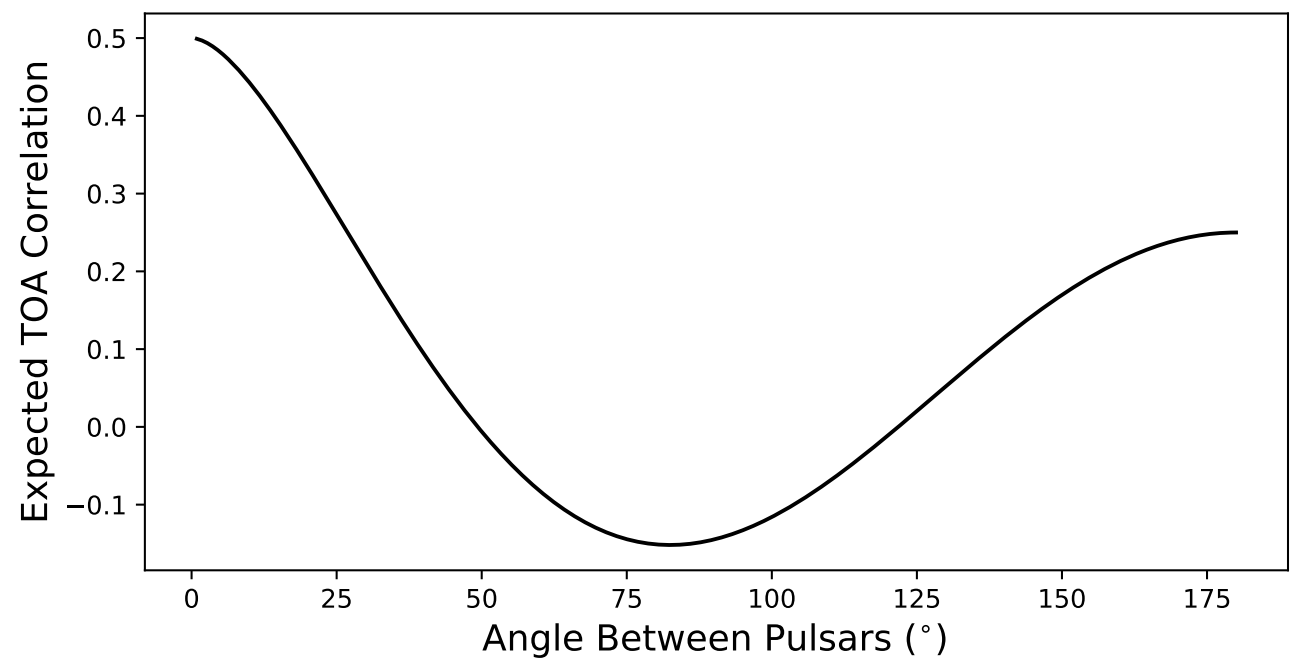

Figure 1.11 Hellings-Downs curve based on Eq. 1.14. This shows the expected correlations in pulsar times of arrival (TOAs) due to a stochastic GW signal. The expected correlation is dependent on the angle between each pair of pulsars on the sky.

is therefore necessary for a PTA to have many MSPs spread across the entire sky so as to have both a large number of pulsar pairs as well as a good distribution of angular separations between them.

At the time of writing there are four major PTA efforts, the North American Nanohertz Observatory for Gravitational Waves (NANOGrav; McLaughlin, 2013), the European Pulsar Timing Array (EPTA; Kramer \& Champion, 2013), the Parkes Pulsar Timing Array (PPTA; Hobbs, 2013), and India Pulsar Timing Array (Joshi et al., 2018). Collectively these four PTAs make up the International Pulsar Timing Array (IPTA; Manchester \& IPTA, 2013). Efforts are also underway in South Africa with MeerKAT and the Square Kilometer Array (SKA) (Bailes et al., 2016) and in China with the Five-hundred-meter Aperture Spherical Telescope (FAST) (Lee, 2016) to start PTA experiments. 
Until recently, efforts to detect the SGWB so far have only been able to place upper limits on its strain amplitude (e.g. Shannon et al., 2015; Arzoumanian et al., 2016; Babak et al., 2016; Arzoumanian et al., 2018b). However, recently the NANOGrav collaboration found strong evidence of a common-spectrum stochastic process, meaning that a common red noise feature was found among the MSPs. If this process is due to GWs, the median strain would be $1.92_{-0.55}^{+0.75} \times 10^{-15}$ at a fre-

quency of $1 \mathrm{yr}^{-1}$ (Arzoumanian et al., 2020b). However, a robust indication of the SGWB, the quadrupolar spatial correlations between the pulsars (e.g. the HellingsDowns correlations), was not detected. This result utilizes timing data for 45 MSPs with timing baselines ranging from 3 to 12.5 years. Recent work by Pol et al. (2020) have used this result to predict that a significant detection of the SGWB will be made by NANOGrav once the longest timing baselines have reached between 15-17 years, a milestone that will be reached sometime in the next 1-3 years.

\subsection{Observing Pulsars}

As described above, observations of pulsars can be used for a number of interesting experiments and studies. However, the nature of pulsar timing is complex, and for the most precise pulsar timing experiments, it is important to verify that data analysis and results pipelines are accurate. Many parameters within a pulsar timing model may be covariant with each other, such as DM and pulse profile evolution parameters, which are both dependent on $\nu$. Other effects that may cause delays in the TOAs, such as scattering, are not modeled by specific parameters in 
a pulsar timing model which then add noise or may be absorbed by other timing model parameters (e.g. Lam et al., 2019; Turner et al., 2020).

If unaccounted for, it is possible, though unlikely (Alam et al., 2020b), that some of these processes may absorb some of the GW signal, which could change the inferred spectrum or amplitude of the SGWB. Unlike other GW detectors such as LIGO, a GW signal is always present in pulsar data, even if we cannot characterize it, so there is no analogous "noise-only" detector model to compare to in a PTA. This means we must try to model noise in our data while a GW signal is present. Many methods are already currently in use to account for these processes, such as uncertainties in models of the Solar System ephemerides that are used to barycenter TOAs (Vallisneri et al., 2020). However, since we do not know the properties of an NS, the ISM, or a GW signal a priori, it is important to utilize simulated data to verify that we are not modeling out any of the GW signal that is present.

\subsubsection{Signal Propagation Path}

There are a number of steps in the path that pulsar signals propagate through on their way to Earth. As described in $\$ 1.1 .2$, pulses are emitted in the magnetosphere of the pulsar where, depending on the height the emission, and subsequently the radio frequency of the emission, the profile shape will vary. In modern pulsar timing, delays from the effects of profile evolution are quantified by so called Frequency-Dependent, or FD, parameters, which are polynomial coefficients in logfrequency space (Zhu et al., 2015; Arzoumanian et al., 2016). The time delays are 
calculated as

$$
\Delta t_{\mathrm{FD}}=\sum_{i=1}^{n} c_{i} \ln \left(\frac{\nu}{1 \mathrm{GHz}}\right)^{i}
$$

where $c_{i}$ are the polynomial coefficients in time units, or the FD parameters, such that $c_{1}=\mathrm{FD} 1$ and so on, $n$ is the number of coefficients, and $\nu$ is the center frequency of each frequency channel.

As the pulse then propagates through the ISM, it is subjected to dispersion, scattering, and scintillation, as described in $§ 1.2$. The pulse profile shape and how it changes with radio frequency, as well as the effects of the ISM, are radio-frequency dependent, and as such there may be a high degree of covariances between measurements of these effects. For studies involving both the ISM and precision pulsar timing, quantifying these covariances is important for characterizing the ISM as well as the noise in the pulsar timing data. However, this is difficult to do with real pulsar observations, since we do not know a priori what these parameters should be.

After a pulse arrives at Earth, it is detected by a radio telescope at some observing frequency based on the telescope receiver. Since pulsars are weak radio emitters, these receivers must be very sensitive, however there is still some amount of noise in the data from white noise fluctuations in the telescope reciever system and the background radiation from the sky (e.g. Haslam et al., 1981). Of particular interest is the noise variance of the receiver, $\sigma_{S}$, as this puts limit on how strong the pulse must be to be detectable. As discussed by numerous authors (see, e.g. 
Lorimer \& Kramer, 2004), the radiometer noise variance can be calculated as

$$
\sigma_{S}=\frac{T_{\text {sys }}+T_{\text {sky }}}{G \sqrt{n_{\mathrm{p}} t_{\mathrm{obs}} \Delta f}},
$$

where $T_{\text {sys }}$ is the system temperature, $T_{\text {sky }}$ is the sky temperature, $G$ is the telescope gain in units of $\mathrm{K} \mathrm{Jy}^{-1}, n_{\mathrm{p}}$ is the number of polarizations, $t_{\mathrm{obs}}$ is the telescope integration time, and $\Delta f$ is the bandwidth of the observation. After being observed at the telescope, data are digitized by a backend and saved in a standard astronomical data file format that can easily be processed by data analysis software.

\subsubsection{Signal Simulation}

The path that these pulses takes from the pulsar through to being recorded at a radio telescope is a long and complex one, with many effects that must be accounted for. Thus being able to simulate the complex signals is useful, as it allows for not only an exploration of covariances, in particular between radio frequency-dependent effects, but also for testing data processing and analysis pipelines. Further, if such signal simulation can be done in a modular and intuitive manner, it becomes not only a scientific simulation tool, but a tool that may be used by students for educational purposes, as simulated data may be much easier to work with than actual data. An extensive simulation software package would also allow for the simulation of any combination of different effects, which could be turned on and off at will by the user, to better explore how different effects impact each other and pulsar timing.

At the time of writing, one of the most widely used and easy to learn coding 
languages in astronomy is Python. Python is a free, object-oriented programming language with a large open-source community that gives access to many large and well-maintained scientific and mathematical tools, such as numpy ${ }^{4}$ (Van Der Walt et al., 2011) and scipy ${ }^{5}$ (Jones et al., 2001). Well-documented, object-oriented software is not only easy to read and understand, but also easy for other users and developers to add features to, resulting in living code that is constantly being improved by a wide user community.

While some software already exists to simulate pulsar signals, such as the fake program in the sigproc ${ }^{6}$ software package (Lorimer, 2011), many effects, such as scattering and profile evolution, are not included. The development of a comprehensive pulsar signal simulation software is therefore of great interest to the broader pulsar and radio astronomy community. To this end the Pulsar Signal Simulator ${ }^{7}$ (PSS) Python package has been developed to create a comprehensive, modular, and easy to use pulsar simulation for a wide community of interested scientists and students. Further discussion of the functionality of the PSS and example usage of it for scientific simulations is discussed further in Chapter 4.

\subsection{Thesis Outline}

The rest of this dissertation is organized as follows. In Chapter 2 we will go over the pulse emission processes of RRATs and the different methods of constraining

\footnotetext{
${ }^{4}$ https://numpy.org/

${ }^{5}$ https://www.scipy.org/

${ }^{6} \mathrm{http}: / /$ sigproc.sourceforge.net

${ }^{7}$ https://github.com/PsrSigSim/PsrSigSim
} 
them. We then preform an analysis of the single pulses from three RRATs. While we find that we cannot explain all of the properties we find, we do not find evidence that their pulsed emission is due to giant pulses or the release of energy that is stored in the pulsar magnetosphere.

We then go on to look at noise processes in the precision pulsar timing of MSPs in Chapter 3. We measure scintillation parameters from multi-hour continuous observations of seven MSPs included in the NANOGrav PTA and are able to not only constrain the different noise contributions to the pulsar timing residuals, but also characterize the ISM along the LOS to each pulsar. We then discuss the merits of simulated data various aspects of pulsar timing and provide a scientific case study in Chapter 4. Here we show not only that the PSS can simulate realistic data, but also its usefulness is exploring the covariances between different frequency-dependent effects. Finally in Chapter 5 we present the main conclusions from these studies and discuss future work that may be done in a variety of fields. 


\section{Chapter 2}

\section{Radio Properties of Rotating Radio Transients: Single-pulse Spectral and Wait-time Analyses}

\subsection{Introduction}

Pulsars are useful for many astrophysical studies and efforts to increase the number of known pulsars are ongoing. While most pulsars are discovered in the Fourier domain, single-pulse searches have led to many interesting discoveries. Singlepulse searches of archival Parkes Multibeam data have led to both the discovery of rotating radio transients (RRATs; McLaughlin et al., 2006) and fast radio bursts (FRBs; Lorimer et al., 2007). RRATs, a subclass of pulsars, are often not detected in the Fourier domain as their emission is quite sporadic, with only a few detectable pulses per hour ${ }^{1}$ (e.g. Keane et al., 2011), and therefore are preferentially discovered with single-pulse searches. However, since RRAT emission has underlying periodicity, one can time them using techniques similar to those used for normal pulsars (McLaughlin et al., 2009). FRBs are notable for being seen as bright, and highly dispersed pulses of radio emission and thus can only be discovered through single-

Published as B. J . Shapiro-Albert et al. 2018, ApJ, 866, 152.

Contributing authors: M. A. McLaughlin, E. F. Keane

${ }^{1}$ see http://astro.phys.wvu.edu/rratalog/ 
pulse searches. FRB 121102 is the sole FRB known to repeat, but has no obvious underlying periodicity (Spitler et al., 2016).

The reason RRATs have such sporadic emission is unknown, but some theories have been put forward to explain this phenomenon. These include fallback of material from a supernova debris disk (Li, 2006) or interference of an asteroid belt around a pulsar (Cordes \& Shannon, 2008). Alternatively, RRATs may be part of the standard canonical pulsar model but exhibit extreme nulling (Wang et al., 2007).

The sources of FRBs remain an open topic of discussion. FRBs are generally considered to be of extragalactic origin given their larger than expected dispersion measures (DMs; e.g. Bhandari et al., 2018). FRB 121102 has been linked to a host galaxy (Chatterjee et al., 2017) confirming its extragalactic origin. Many emission mechanisms have been proposed, including active galactic nucleus emission (e.g. Vieyro et al., 2017), giant flares from magnetars (Lyubarsky, 2014), ejection of relativistic high-energy shells from a compact object (Waxman, 2017), or the ejection of relativistic jets into surrounding plasma (Romero et al., 2016). Recently, Michilli et al. (2018) found that FRB 121102 has a large rotation measure, indicating the source must be located in an extreme magnetic environment. Additionally, most FRB's also exhibit purely broadband emission, with FRB 121102 also showing narrowband emission (Spitler et al., 2016), and FRB 170827 (Farah et al., 2018) showing an interesting narrowband frequency structure. Other FRBs, including FRB 121102, show broadband emission over just part of the band which could be an effect of scintillation (Spitler et al., 2018). 
Previous work by Keane (2016) and Rane \& Loeb (2016) has looked into potentially mis-categorizing FRBs as RRATs if they have only been discovered from a single-pulse. While no obvious evidence that any of these single-pulse RRATs should be categorized as an FRB has been found, at DMs near the maximum expected from the Milky Way along a particular line of sight, there is potential for this mis-categorization. Our work does not address this issue as we consider only RRATs that have been observed with many bright pulses within the Galaxy.

We can learn about the emission mechanisms of RRATs by searching for periodicities in pulse arrival times as in Palliyaguru et al. (2011) who found evidence for periodicities in some RRATs on timescales ranging from 1.4 hrs to $\sim 5$ yrs. We can also analyze the pulse-amplitude or pulse-energy distributions, as these are useful metrics for comparing different pulsar emission modes. While standard pulsar emission often results in log-normal distributions (Burke-Spolaor et al., 2012), so-called "giant pulses (GRPs)" exhibit power-law distributions and are thought to be due to a different emission mechanism (Cordes et al., 2004; Karuppusamy et al., 2010). Additionally, we can look for correlations between the wait-time with the pulse flux density (Cui et al., 2017) to examine the emission mechanism.

While most FRBs are single events, FRB 121102 shows large variations in the pulse structure with both frequency and time, exhibiting both broad- and narrowband emission, with spectral indices ranging from -10 to +14 at $1400 \mathrm{MHz}$ (Spitler et al., 2016). Similarly, single-pulse spectral index studies on Crab GRPs have found a large spread from $\sim-10$ to $\sim+10$ at $1400 \mathrm{MHz}$ for the main pulse (Karuppusamy et al., 2010). However, while the spread is large, the mean spectral index of the Crab 
pulsar's main pulse, determined from a Gaussian fit of the spectral index distribution, was found to be $-1.4 \pm 3.3$, where the uncertainty is the standard deviation of the Gaussian. Though not well constrained, this is consistent with previous results from both Bates et al. (2013), who used pulsar population simulations to find that the mean spectral index for pulsars is $-1.41 \pm 0.06$, and also from Jankowski et al. (2018) who found a mean pulsar spectral index of $-1.60 \pm 0.03$ for 348 pulsars that follow a simple power-law spectrum. In general, almost all pulsars have been found to have negative spectral indices and exhibit broad-band emission (e.g. Lorimer et al., 1995; Maron et al., 2000; Jankowski et al., 2018). This type of emission is consistent with studies of the Crab, but inconsistent with FRB 121102, which has been observed to have narrow-band emission, and is poorly described by a single spectral index power law (Law et al., 2017).

The standard method of computing the spectral indices of pulsars generally involves fitting a power law to the flux density of the folded profile at multiple different frequency bands across a wide bandwidth (e.g. Lorimer et al., 1995; Maron et al., 2000; Jankowski et al., 2018). However, folding the pulse profiles loses information, such as pulse-to-pulse profile variation. Similarly if the profile is summed over many frequency subbands then frequency structure can be lost. Similarly, the flux density of the folded profile is often only taken over a single epoch, and thus does not account for any variation on larger timescales.

It has been suggested that, since RRATs are usually analyzed by their singlepulses, they present the opportunity for comparison to FRB 121102. Most RRAT single-pulses have peak flux-density values of $\sim 0.1-1$ Jy (McLaughlin et al., 
2009), similar to the peak flux density of many FRBs, which allows us to determine the spectral properties of the single-pulses in a similar way. However, to obtain statistically significant results, we must analyze a large number of single-pulses. Since most RRATs have a low burst rate, many epochs of observation are necessary to observe a large enough sample of pulses. Analyzing data taken over many years has the added benefit of allowing us to account for both single-pulse spectral index and single-pulse energy variation on long timescales.

In the following we analyze 11 years of RRAT timing data, as well as the Parkes Multibeam Pulsar Survey (PMPS; Manchester et al., 2001) data, and conduct a single-pulse and wait-time analysis on three RRATs. We also include a single 7.5 hour observation of PSR J1819-1458 by the 100-m Green Bank Telescope (GBT) for additional single-pulse analysis. We describe the different observations in $§ 2.2$. Our single-pulse search and analysis methods are described in $\S 2.3$. We discuss the results of our analyses, presenting the RRAT single-pulse spectral results, wait-time distribution results, and the pulse energy distribution results in $\S 2.4$. Concluding remarks and discussion on future studies are given in $§ 2.5$.

\subsection{Data}

The RRAT data come from two main sets, the PMPS (Manchester et al., 2001), and follow-up observations for RRAT timing taken with Parkes between MJD 52863 (2003 August 12) and MJD 55857 (2011 October 23). An additional 7.5 hour observation of PSR J1819-1458 taken with the GBT is also analyzed. 
The PMPS observations were taken with the 13-beam receiver on the $64 \mathrm{~m}$ Parkes Radio Telescope between 1998 January and 2002 February. Each observation was 35 minutes in length. The bandwidth for the PMPS data was $288 \mathrm{MHz}$ split into 96, $3 \mathrm{MHz}$ frequency channels centered on $1374 \mathrm{MHz}$. The data were taken with a sampling rate of $250 \mu$ s with 1-bit precision (Manchester et al., 2001).

The majority of the rest of our data are the same as presented in McLaughlin et al. (2009), but with an additional two years of observations. All observations are between 0.5 and 2 hr long. These data were taken with central beam of the 13-beam receiver on the $64 \mathrm{~m}$ Parkes Radio Telescope. Most of the observations were taken with a central frequency of $1390 \mathrm{MHz}$, a bandwidth of $256 \mathrm{MHz}$ and 512 frequency channels, and were taken with a sampling rate of $100 \mu$ s with 1-bit precision. A minority of RRAT timing observations were taken with the $10-50 \mathrm{~cm}$ receiver, which has a bandwidth of $64 \mathrm{MHz}$ around $685 \mathrm{MHz}$ and a bandwidth of $768 \mathrm{MHz}$ around $3 \mathrm{GHz}$, and the $\mathrm{HOH}$ receiver, which has a bandwidth of $576 \mathrm{MHz}$ and a central frequency of $1500 \mathrm{MHz}$. For consistency in our data set we have ignored these data in our analysis.

In addition to the data from Parkes, we have also separately analyzed a $7.5 \mathrm{hr}$ observation of PSR J1819-1458 taken with the GBT on MJD 54557 (2008 April 1). This observation was taken with a bandwidth of $800 \mathrm{MHz}$ and a center frequency of $2 \mathrm{GHz}$. These data were taken at a sampling rate of $81.92 \mu$ s with 8-bit precision. However we have only the dedispersed, frequency-scrunched, time series for this observation and thus have no spectral information. 


\subsection{Methods}

We first describe the methods used to determine the single-pulse spectral indices. We then analyze simulated data using these same methods to determine if an injected spectral index could be recovered. We then determine which RRATs are viable candidates for our analysis.

Additionally, we analyze the distributions of pulse wait-times. If the emission on short timescales is purely random, these distributions will be exponential. However, deviation from an exponential suggests that there may be some periodicities to the emission on short timescales. We test this by fitting a variety of distributions in various combinations and performing multiple goodness-of-fit tests on these.

Finally we look for correlations in the flux density of the single pulses with wait time. An increase in flux density with wait-time could suggest that the emission is due to a build-up of energy in the pulsar magnetosphere.

\subsubsection{Single-pulse Spectral Index Analysis}

\subsubsection{Identification of Single pulses}

To identify single pulses from RRATs, we use the same method as described in McLaughlin et al. (2009). Using SEEK and other packages in SIGPROC ${ }^{2}$ (Lorimer, 2011) to search for pulses at a $5 \sigma$ threshold, the data are dedispersed with DM of both $0 \mathrm{pc} \mathrm{cm}^{-3}$ and the known DM of the RRAT (see Table 2.1). SEEK may underestimate the signal-to-noise $(\mathrm{S} / \mathrm{N})$ of a single-pulse (Keane \& Petroff, 2015).

\footnotetext{
${ }^{2}$ http://sigproc.sourceforge.net/
} 
However the $\mathrm{S} / \mathrm{N}$ returned by SEEK is used just for initial thresholding purposes, and the actual $\mathrm{S} / \mathrm{N}$ of the pulse is calculated during the fitting and calibration steps of our analysis. It is possible that we may not detect some weak pulses, but we estimate that even in a worst-case scenario where, assuming the pulses are randomly emitted, a pulse will fall into every underestimated phase of the SEEK boxcar, we will only miss only one out of every five pulses detected with an $\mathrm{S} / \mathrm{N}$ of $7 \sigma$ or less. This is at most $\sim 3 \%$ of the pulses found for a RRAT with a $\sim 1$ s period and a pulse width of $\sim 10 \mathrm{~ms}$. As the goal of this work requires only a large population of bright pulses, this should not significantly impact our results.

Pulse candidates at both DMs are then compared and any pulses detected with a higher $\mathrm{S} / \mathrm{N}$ at a $\mathrm{DM}$ of $0 \mathrm{pc} \mathrm{cm}^{-3}$ are discarded as radio frequency interference (RFI), as pulses from the RRAT will be brighter at the true DM. We set a minimum detection threshold of $5 \sigma$ for each pulse.

As an additional guarantee that we have filtered out all RFI, we only take pulses whose times of arrival (TOAs) based on the brightest pulse in an observation occur within $5 \%$ of the expected phase. For the purposes of our wait-time analysis, we then round the wait-time such that it is an integer number of pulse periods. After this filtering, each pulse is also visually inspected and any remaining RFI is manually discarded. 

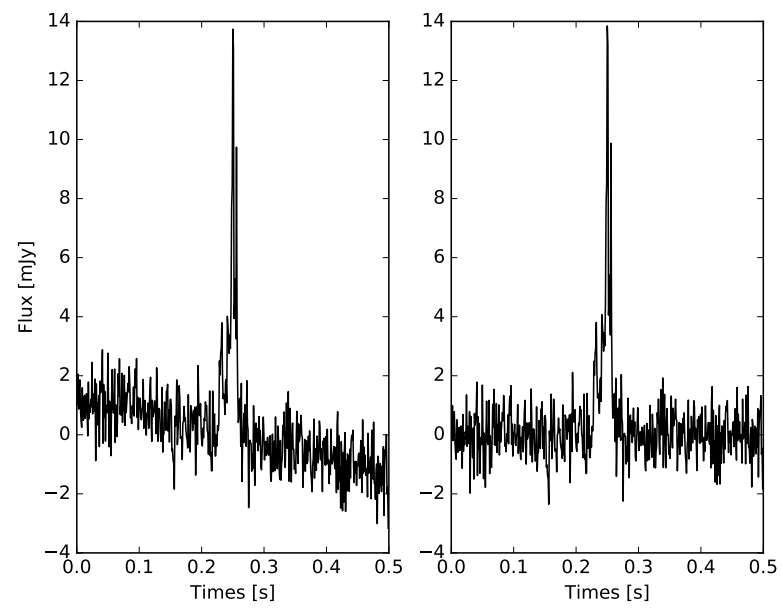

Figure 2.1 Single pulse from the GBT observation of PSR J1819-1458. Left: the pulse after calibration, but before the Gaussian Process Regression (GPR) has been implemented. We see that the baseline is obviously sloped. Right: the pulse postGPR. We see that the slope of the baseline has been eliminated without compromising the pulse profile.

\subsubsection{Fitting Single-pulse Templates}

After the pulses have been filtered using the methods described above, we take $0.5 \mathrm{~s}$ of dedispersed data split into 512 time bins around each pulse. This is done to reduce the amount of time necessary to process the data. From simulations (see $§ 2.3 .2)$ we find the optimal number of subbands for each set of observations. Each single-pulse from the timing data of McLaughlin et al. (2009) is split into 16 subbands. When dedispersing the PMPS observations (Manchester et al., 2001), each pulse is split into 12 subbands. Since we must be able to detect the pulse in each subband, we take only pulses detected above an $\mathrm{S} / \mathrm{N}$ threshold of $8 \sigma$. In order to determine the flux density of each pulse, we calibrate the data using the 
radiometer equation (Lorimer \& Kramer, 2004),

$$
\Delta S_{\mathrm{sys}}=\frac{\beta T_{\mathrm{sys}}}{G \sqrt{n_{\mathrm{p}} t_{\mathrm{obs}} \Delta f}}=C \sigma_{\mathrm{p}}
$$

where our system temperature, $T_{\text {sys }}$, is the receiver temperature, $T_{\text {rec }}$, plus the sky temperature, $T_{\text {sky }} \cdot T_{\text {sky }}$ is determined at the center frequency of each subband scaling from the $408 \mathrm{MHz}$ all sky survey of Haslam et al. (1981) assuming a spectral index of -2.6 (Lawson et al., 1987). For the Parkes Multibeam Receiver, $T_{\text {rec }}$ is taken to be 28 K (2017 October 14 version of the "Parkes Radio Telescope Users Guide"). For the GBT S-band receiver, $T_{\text {rec }}$ is taken to be $20 \mathrm{~K}$ (2017 February 14 version of "Observing With The GBT"). For both Parkes and the GBT, spillover and atmospheric contributions to $T_{\text {sys }}$ are negligible at the frequencies considered here. The $\beta$ factor accounts for loss due to 1-bit digitization and is $\sqrt{\pi / 2}$ for the Parkes observations, and $\sim 1$ for the GBT observation as it records with 8-bit precision. Our data are multiplied by the resulting conversion factor, $C$, in order to convert arbitrary units of flux density to mJy. Finally, $\sigma_{\mathrm{p}}$ is the standard deviation of the off-pulse region.

We then remove any variations in the baseline that occur due to RFI or instrumental effects. Most of our data were not badly affected by these variations, but, as shown in the left panel of Figure 2.1, removal of these trends was necessary in some cases. We removed these variations by Gaussian Process Regression (GPR; Rasmussen, 2006) which is implemented through the python package GPy ${ }^{3}$. We fit

\footnotetext{
${ }^{3}$ https://sheffieldml.github.io/GPy/
} 
the baseline only in the off-pulse region, determined as the area outside of twice the full width at half max (FWHM) of the pulse measured from its peak. This is visually checked to make sure that no part of the pulse in within this off-pulse region. Only variations more than $1 \sigma$ away from a zero mean with length scales of at least $\sim 0.05 \mathrm{~s}$ are considered. The fit over the baseline is then subtracted from the pulse to remove these variations. An example of a single-pulse after GPR is shown in the right panel of Figure 2.1.

Once the pulse has been calibrated and undergone GPR, we fit a template profile to the pulse using a least-squares minimization technique. Performing GPR on each pulse before the fitting is necessary because the pulse in each subband is usually noisy and has little flux density, so having a prior template that is immune to this noise allows for better subband fitting. We do not subtract this baseline from each subband, as we fit only the amplitudes of each component of our template in each subband. To do this fitting, we use the leastsq function in the python package scipy $^{4}$ (Jones et al., 2001).

One of the most well studied RRATs, PSR J1819-1458, has three components in its profile (Karastergiou et al., 2009), so we allow our profile template to fit up to three Gaussian components to each single-pulse. This also helps to account for variable small-scale structure in the pulse profile. Our total profile integrated over frequency is fit by

$$
P(t)=\sum_{i=1}^{3} A_{i} \exp \left(-\frac{\left(t-t_{p_{i}}\right)^{2}}{2 \sigma_{i}^{2}}\right)
$$

\footnotetext{
${ }^{4}$ https://www.scipy.org/
} 
where $t$ is the time of the pulse, $i$ the $i$ th pulse component, and $A$, the amplitude, $t_{p}$, the time of the pulse peak, and $\sigma$, the width, are the free parameters of the pulse profile. After the first component is fit, we subtract the fit from the data and a second component is fit from the residual. The three-component fit is made by subtracting the two-component fit from the data and then fitting a third component from the residual. The third component is fit even if no significant secondary component is found. If the pulse does not have a significant second or third component, the Gaussian component that is fit will have zero amplitude.

This fitting method estimates an error matrix for the template using the partial derivatives of the Gaussian components. Since some of our pulses are weak, in order to add a second or third Gaussian component to the template we require the reduced chi-squared value, $\chi_{r}^{2}$, to be at least $10 \%$ better than a fit with fewer components.

The standard deviation used for the $\chi_{r}^{2}$ of each template is taken as the root mean square of the off-pulse around that single-pulse. We define the on-pulse region as twice the FWHM of the single Gaussian fit determined by fitting a single Gaussian component to the pulse using the least-squares fitting method described above. The region outside of this is the off-pulse.

\subsubsection{Obtaining a Single-pulse Spectral Index}

To fit a spectral index to each single pulse, we fit the amplitude, or flux density, of the pulse in each subband. We assume that the number of components, the phase, and the width of each component do not change between the subbands and our 
composite fit. We also assume that we have averaged over many scintles in each subband (see §2.3.3) so our analysis is not limited by scintillation. We then perform the same calibration described above on each subband. Our least-squares fitting in the subband fits only for the amplitude of each component. The uncertainty in this fit is again taken from the error matrix and is based on the partial derivatives.

However, the partial derivatives of a Gaussian increase dramatically if components are either very narrow or very close to each other. This is due to the error inherent in our template fitting, and is not physical. We check for this by testing if the fitting error on the pulse amplitude is greater than $1000 \%$, as this is where this issue manifests most clearly. If the partial derivatives meet this criterion, we account for it by assuming a 50\% template fitting uncertainty. This down-weights the flux density in the subband so that it has smaller impact on our fit spectral index.

We then integrate the Gaussian template of the pulse over the half second of data we record to obtain an estimate of the flux density in each subband,

$$
S(A, \delta, \sigma)=\int_{0}^{0.5} P(t) \mathrm{d} t
$$

where $P(t)$ is defined as in Eq. 2.2. We note that, while integrating the template minimizes the amount of noise included in the flux density, it is likely to underestimate the true flux density of the pulse, as small pulse structures may not meet our $\chi_{r}^{2}$ constraint. 
Table 2.1 RRAT Parameters

\begin{tabular}{cccccccc}
\hline \hline Name & $\begin{array}{c}\text { Period } \\
(\mathrm{s})\end{array}$ & $\begin{array}{c}\text { DM } \\
\left(\mathrm{pc} \mathrm{cm}^{-3}\right)\end{array}$ & $\begin{array}{c}\text { R.A. (J2000) } \\
(\mathrm{hms})\end{array}$ & $\begin{array}{c}\text { Decl. (J2000) } \\
\left({ }^{\circ} \prime \prime\right)\end{array}$ & $\begin{array}{c}T_{\text {obs }} \\
(\mathrm{hr})\end{array}$ & $\begin{array}{c}N_{5 \sigma} \\
(\mathrm{pulses})\end{array}$ & $\begin{array}{c}\text { Pulse Rate } \\
\left(\mathrm{h}^{-1}\right)\end{array}$ \\
\hline PSR J1819-1458 & 4.263 & $196(3)$ & $18: 19: 33.0(5)$ & $-14: 58: 16(32)$ & 44.8 & 1170 (Parkes) & $\sim 26$ \\
& & & & & 7.5 & 937 (GBT) & $\sim 125$ \\
PSR J1913+1330 & 0.923 & $175.64(6)$ & $19: 13: 17.975(8)$ & $+13: 30: 32.8(1)$ & 28.9 & 228 & $\sim 8$ \\
PSR J1317-5759 & 2.642 & $145.3(3)$ & $13: 17: 46.29(3)$ & $-57: 59: 30.5(3)$ & 61.3 & 289 & $\sim 5$ \\
\hline
\end{tabular}

Note. - Parameters for RRATs analyzed in this work. All parameters are from McLaughlin et al. (2009) except those for PSR J1819-1458 which are from Lyne et al. (2009). $T_{\text {obs }}$ is the total time spent on each object and $N_{5 \sigma}$ is the total number of pulses found above a $5 \sigma$ detection threshold.

To calculate the uncertainty of the subband flux density, we take

$$
\sigma_{S}=\left[\sum_{i=1}^{3} 2 \pi\left(\sigma_{i}^{2} \sigma_{A_{i}}^{2}+A_{i}^{2} \sigma_{w_{i}}^{2}\right)\right]^{\frac{1}{2}}
$$

where $A, \sigma$, are defined as above, $\sigma_{A_{i}}$ is the uncertainty in the amplitude, and $\sigma_{w_{i}}$ the uncertainty in the width. We do this for each subband of the pulse and then use a weighted least-squares fit to determine the spectral index of the pulse.

We assume the flux density varies as a power law,

$$
S \propto \nu^{\alpha}
$$

where $S$ is the total integrated flux density of our pulse template in the subband, $\nu$ the center frequency of the subband, and $\alpha$ the spectral index of the pulse. If the amplitude of a Gaussian component of the pulse profile fit is returned as a negative value, it is set to zero. We remove from the spectral index fit any subband where the computed flux density is more than $2 \sigma$ below the mean flux density of the other subbands. 

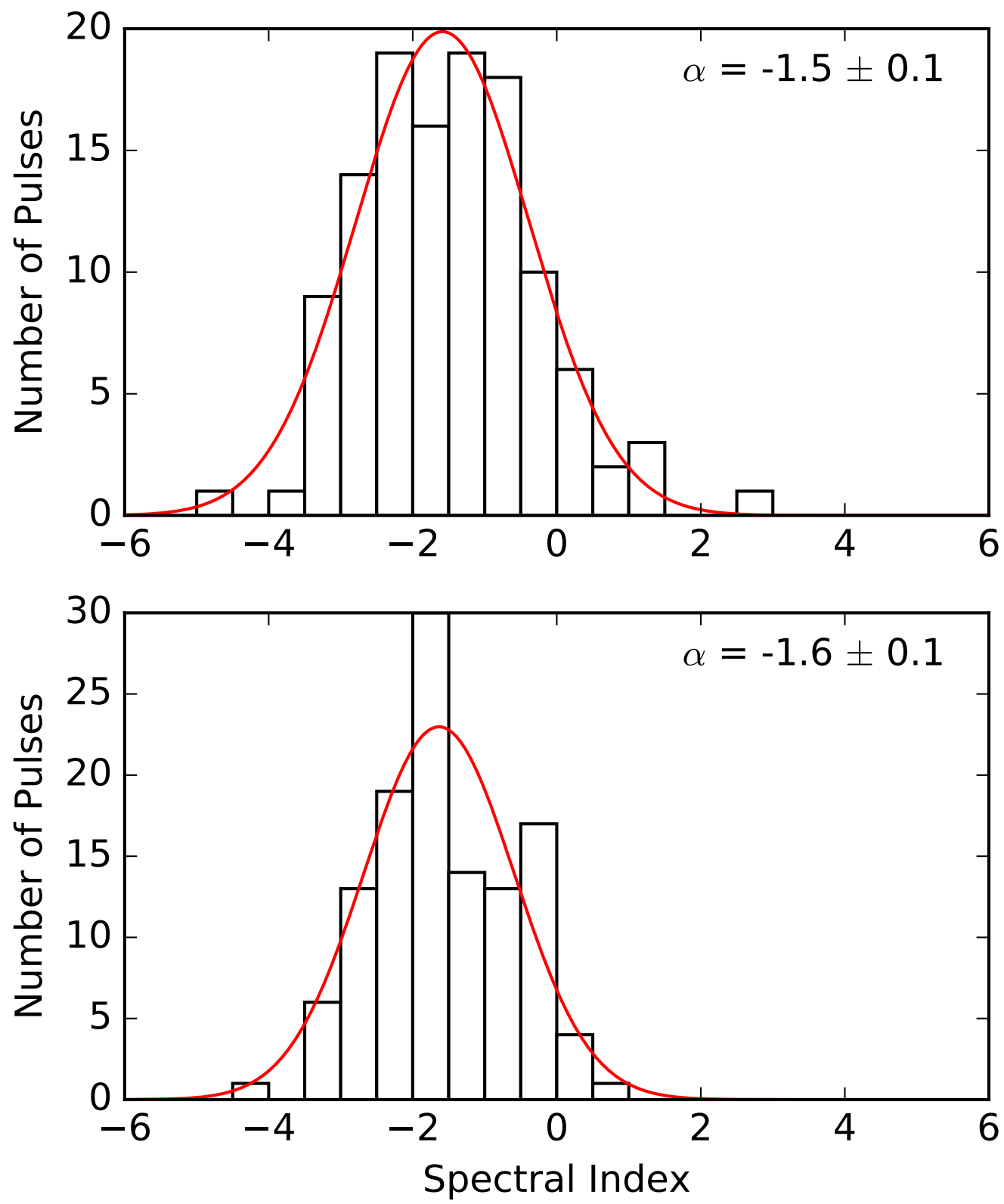

Figure 2.2 Top: distribution of the spectral indices for the simulated PMPS-style data with an input spectral index of -1.5 . The weighted mean spectral index with mean errors is reported in the upper right corner as $\alpha=-1.5 \pm 0.1$ and a Gaussian fit is shown over the histogram. The standard deviation of the Gaussian is 1.2. Bottom: distribution of the spectral indices for the simulated RRAT timing-style data with an input spectral index of -1.5 . The weighted mean spectral index with mean errors is $\alpha=-1.6 \pm 0.1$. The standard deviation of the Gaussian is 1.0. The timing data are more precise since they have more frequency subbands, which accounts for the slightly narrower Gaussian fit to the simulated data. 
While removing these points could bias our fit, our weighted fit will weight subbands with the largest error bars the least. Therefore the removal of these subbands will have a minimal effect on the fit spectral index.

The uncertainty in the fit spectral index of the each single-pulse is given from the covariance matrix produced by the least-squares fit as described above. The mean spectral index is determined by computing the weighted mean of the spectral indices of all of the single-pulses.

\subsubsection{Analysis of Simulated Data}

In order to verify that the methods described in $§ 2.3 .1$ return the correct spectral indices, we simulated data from both the PMPS as well as the RRAT timing observations using the fake function available in SIGPROC-v4.3 (Lorimer, 2011). The simulated PMPS observation has the same parameters as described in $\S 2.2$ and was given a length of $2 \mathrm{~min}$, a period of $1 \mathrm{~s}$, pulse width of $0.01 \mathrm{~s}$, and an injected single-pulse peak $\mathrm{S} / \mathrm{N}$ of 2 . These parameters allowed each pulse to be detected by SEEK with a maximum $\mathrm{S} / \mathrm{N}$ above our threshold of $8 \sigma$. As noted earlier, SEEK can also underestimate the $\mathrm{S} / \mathrm{N}$ of a pulse based on the pulse phase of the boxcar searching algorithm (Keane \& Petroff, 2015). This variation, in addition to the variation due to smoothing the data, returned all 120 pulses with an $\mathrm{S} / \mathrm{N}$ between 9 and $12 \sigma$. We then multiplied each frequency channel by an appropriate scaling factor to inject the simulated data with a spectral index of -1.5 .

After simulation and detection of the single-pulses, we then used the method 
described in $§ 2.3 .1$ to fit a spectral index to each pulse and calculate the mean spectral index. When calibrating the simulated data, we used Eq. 2.1 but took $T_{\text {sys }}=T_{\text {rec }}$ because fake does not simulate the sky temperature and since $T_{\text {sky }} \propto \nu^{-2.6}$ (Lawson et al., 1987), adding this induces a separate spectral index not initially present in our simulated data.

In order to determine if the number of subbands used is significant, the method was repeated with the data split into 6, 12, 24, 48, and 96 subbands. Depending on the steepness of the spectral index of a single-pulse, across any given subband, the spectral index may not be flat. However, for a spectral index of -1.5 , the largest subband tested was $48 \mathrm{MHz}$ wide, which exhibits a change in the spectral index of 0.03 (e.g. from -1.5 to -1.53 ) across one subband. This is much smaller than the uncertainty on any given spectral index as well as on the weighted mean spectral index for the entire distribution. We therefore assume that the spectral index across any given subband is flat.

For each case, the weighted mean spectral index was calculated and a Gaussian was fit with a least-squares method to the distribution of spectral indices. In all cases, the mean spectral index of the pulses was recovered at -1.5 within $1 \sigma$. The reduced chi-squared value for each Gaussian fit was also determined. Finally, we used our analysis software (see $§ 2.3 .1$ ) to determine the actual $\mathrm{S} / \mathrm{N}$ distribution and analytically determined what the distribution of spectral indices should be based on this. Based on the criteria described above, we determined that using 12 subbands accurately recovered our injected spectral index of -1.5 and using more subbands did not improve our recovered values. The distribution of spectral indices for this 
set of simulate data using 12 subbands is shown in the top panel of Figure 2.2.

To simulate the later RRAT timing observations, we use the same observation parameters as in $\S 2.2$ and the same pulsar parameters as with the simulated PMPS data. For this set, 120 single-pulses were found above our $8 \sigma$ threshold with most having a maximum $\mathrm{S} / \mathrm{N}$ value reported by SEEK between 12 and $16 \sigma$. The difference in detection significance between this set of simulated data and the simulated PMPS data is due to the method by which the boxcar used by SEEK smooths the data. To check if the number of subbands used is significant, our method was tested on this simulated data set using 4, 8, 16, 32, 64, and 128 subbands. We used the same criteria described for the simulated PMPS data to determine which number of subbands best recovered our initial values. We found that 16 subbands most closely recovered the injected spectral index, as shown in the bottom panel of Figure 2.2.

The difference in the optimal number of subbands is due to the fact that the even divisors of the total number of subbands (96 for the PMPS data and 512 for the RRAT timing observations) are different. For the rest of our spectral index analysis, we have split the PMPS pulses into 12 subbands, and the pulses from all other observations into 16 subbands.

\subsubsection{RRATs Analyzed in This Work}

After initial testing using the methods described in $§ 2.3 .1$, our work focuses on PSRs J1819-1458, J1913+1330, and J1317-5759 as no other RRATs in our data set had enough detectable bright pulses for a complete analysis (McLaughlin et al., 


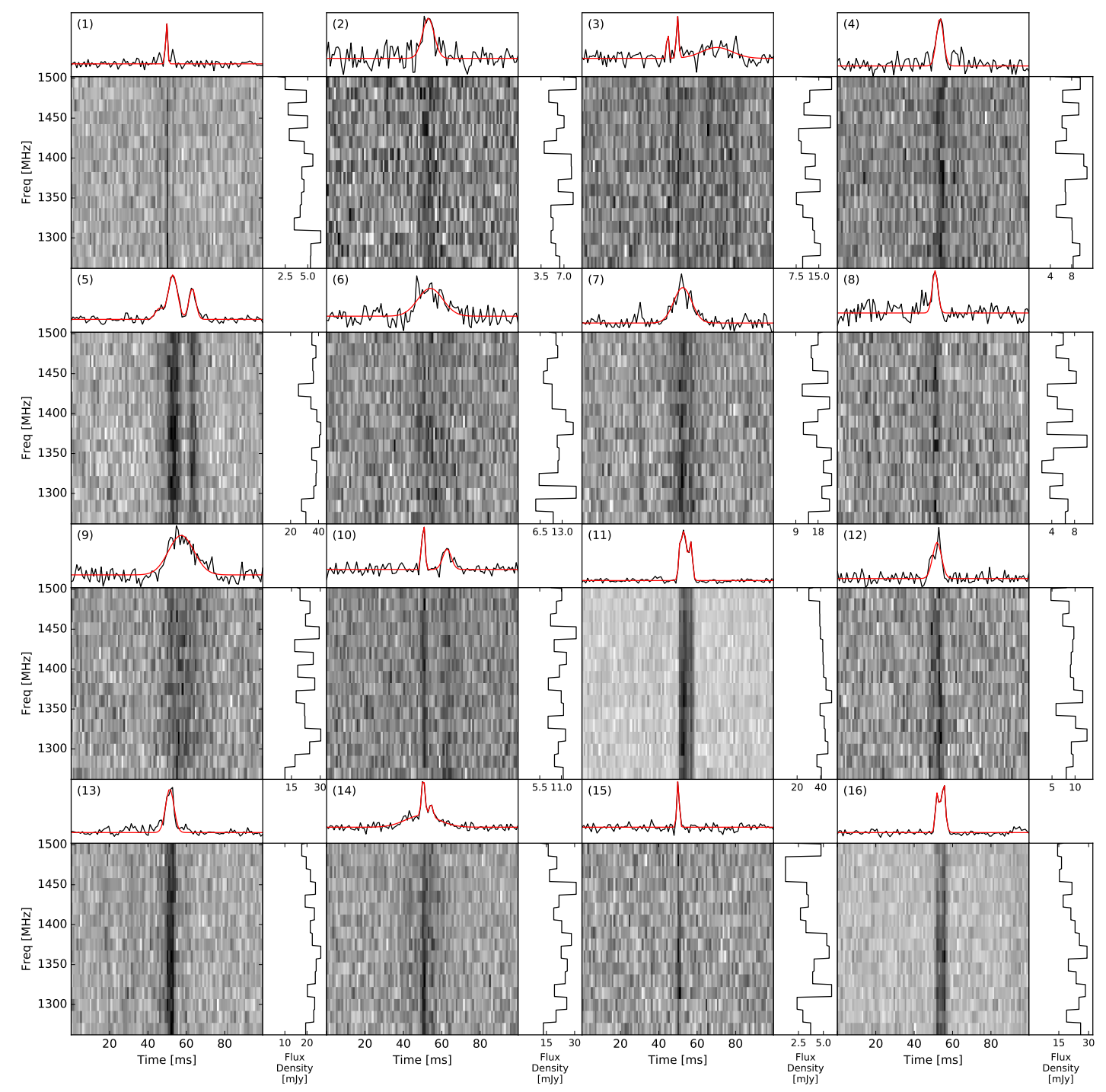

Figure 2.3 Sample of single pulses from PSR J1819-1458 from a single 30 minute Parkes timing observation. The top panel of each plot shows the frequency-summed composite pulse in black, with the Gaussian template fit in red. The dedispersed frequency-time plot for the pulse is shown below the composite pulse panel. To the right is a measure of the mean flux density in mJy of the pulse in each subband. Time and frequency axes are shared by all pulses in the same column and row respectively. This sample illustrates the wide variety of single-pulses we observe. In Figure 2.4 we show the spectral fits for these pulses. 

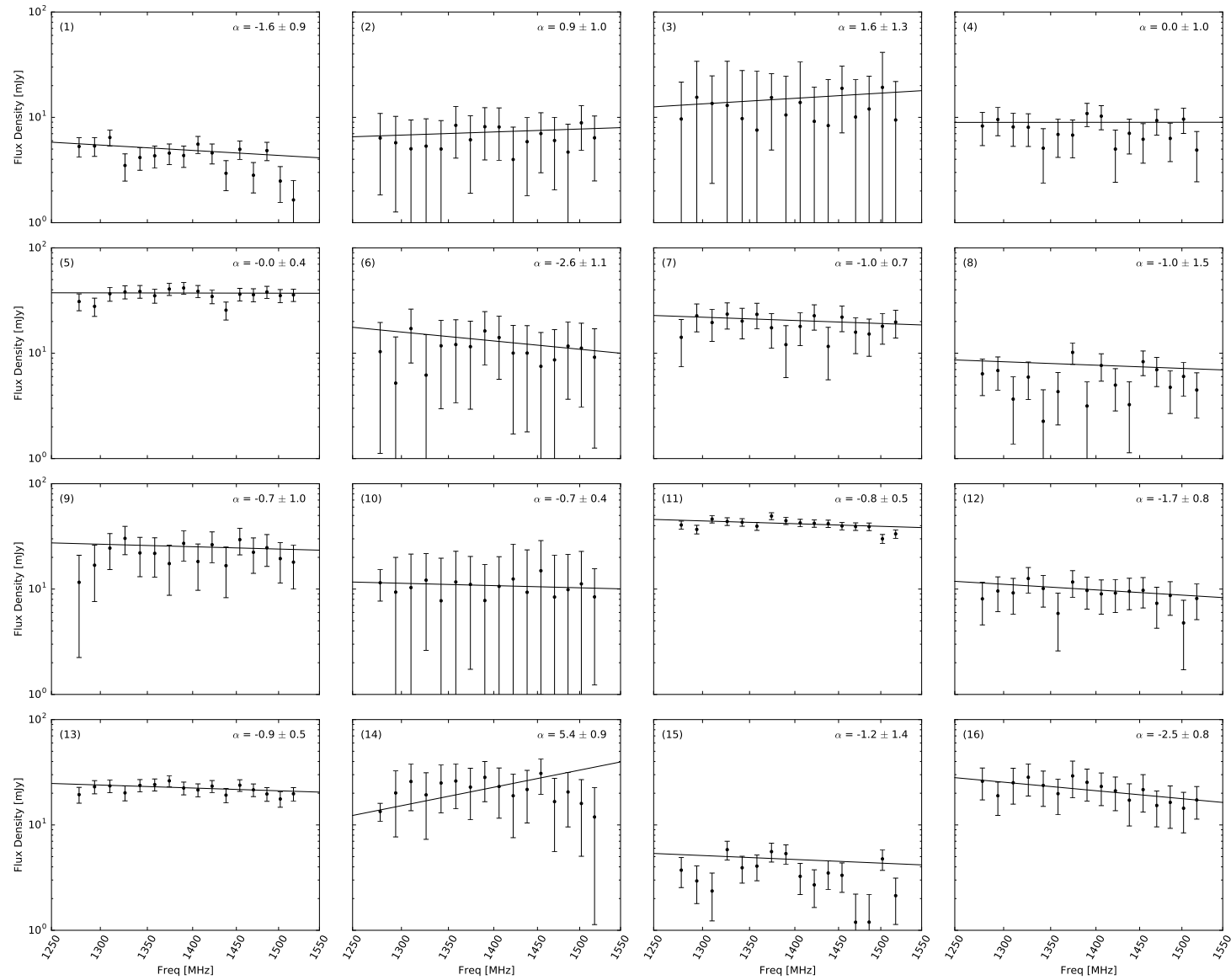

Figure 2.4 Sample of single-pulse spectral index fits from PSR J1819-1458. Each single-pulse spectral index fit can be referenced to the corresponding single-pulse in Figure 2.3. The frequency and flux density scales are the same for each pulse spectral index fit. The scaled mean flux density from Equation 2.5 is plotted against the frequency in $\log$ space. 

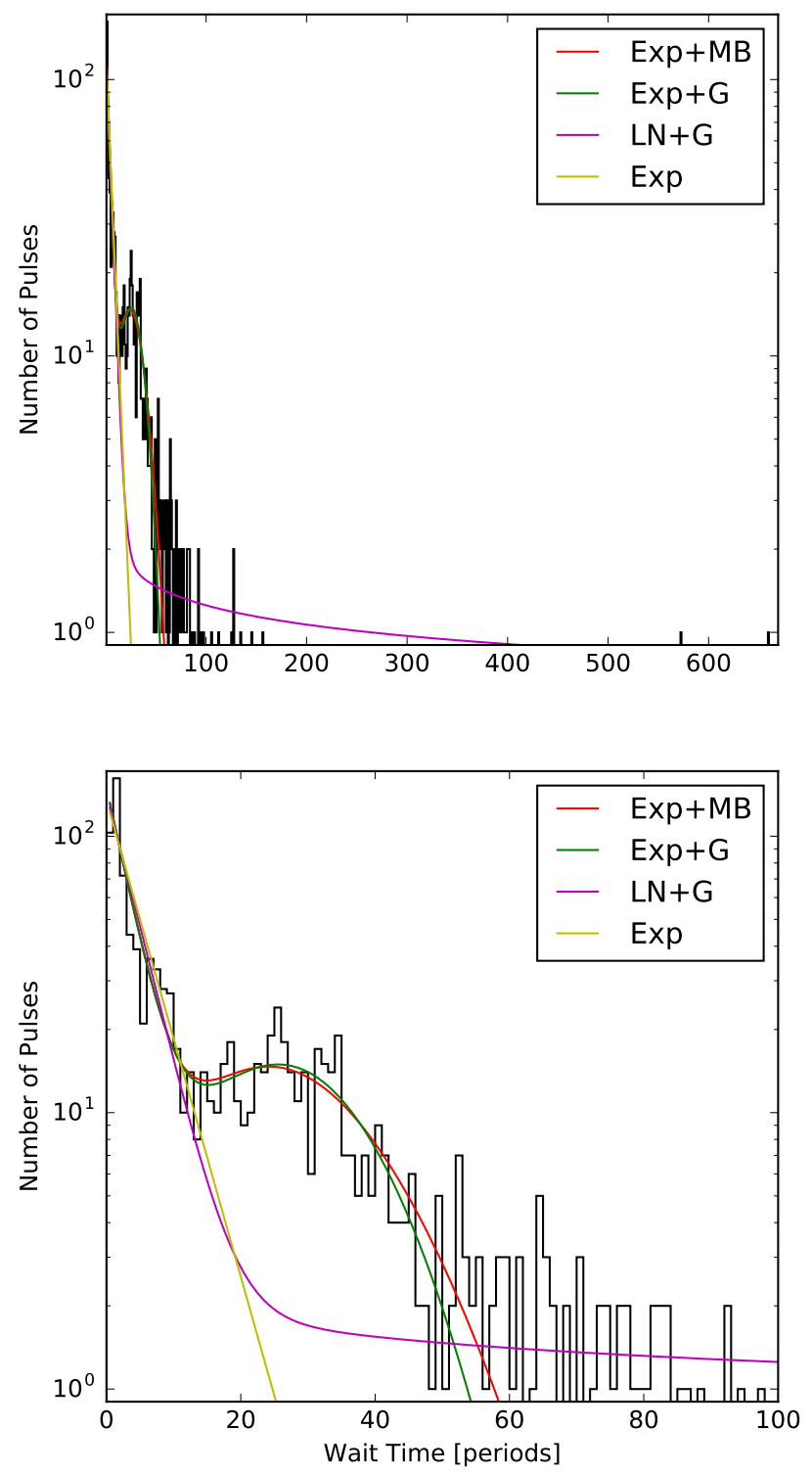

Figure 2.5 Upper panel: distributions of single-pulse wait-times for the Parkes observations of PSR J1819-1458 at L-band. We see that most pulses come within a couple of periods of each other. We also see that there is an extended tail of long wait-times and a small secondary distribution around 25 periods. We fit the full distribution with an exponential plus a Gaussian (Exp+G; green), an exponential plus a Maxwell-Boltzmann distribution (Exp+MB; red), a log-normal plus a Gaussian (LN+G; magenta), and a pure exponential (Exp; yellow). Lower panel: same as the upper panel but zoomed in on the first 100 periods. 
Table 2.2 RRAT Scattering Parameters

\begin{tabular}{cccccccc}
\hline \hline \multirow{3}{*}{ Name } & \multicolumn{3}{c}{ NE2001 } & \multicolumn{3}{c}{ YMW16 } \\
& $\begin{array}{c}\Delta \nu_{\mathrm{d}} \\
(\mathrm{kHz})\end{array}$ & $\begin{array}{c}\Delta t_{\mathrm{d}} \\
(\mathrm{s})\end{array}$ & $\begin{array}{c}\tau_{\mathrm{d}} \\
(\mu \mathrm{s})\end{array}$ & $\begin{array}{c}\Delta \tau_{\mathrm{d}} \\
(\mu \mathrm{s})\end{array}$ & $\begin{array}{c}\Delta \nu_{\mathrm{d}} \\
(\mathrm{kHz})\end{array}$ & $\begin{array}{c}\Delta t_{\mathrm{d}} \\
(\mathrm{s})\end{array}$ & $\begin{array}{c}\tau_{\mathrm{d}} \\
(\mu \mathrm{s})\end{array}$ \\
\hline PSR J1819-1458 & 1.3 & 13 & 80 & 70 & 0.5 & 7 & 30 \\
PSR J1913+1330 & 4.8 & 31 & 20 & 10 & 1.6 & 14 & 20 \\
PSR J1317-5759 & 30 & 52 & 4.3 & 4 & 0.7 & 12 & 1 \\
\hline
\end{tabular}

Note. - Scintillation and scattering parameters for the three RRATs from both NE2001 (Cordes \& Lazio, 2002) and YMW16 (Yao et al., 2017) DM models. We note that $\Delta \nu_{\mathrm{d}}$, $\Delta t_{\mathrm{d}}$, and $\tau_{\mathrm{d}}$ are taken at the center of our band. However, $\Delta \tau_{\mathrm{d}}$ for NE2001 denotes the difference in pulse width due to scattering between the top of the band and the bottom, or the change in $\tau_{\mathrm{d}}$ across our band. The YMW16 $\tau_{\mathrm{d}}$ denotes the scattering at just the center of our band. We do not calculate $\Delta \tau_{\mathrm{d}}$ for the YMW16 model since their values of $\tau_{\mathrm{d}}$ come purely from a scaling law in Bhat et al. (2004) based on DM.

2009). Useful parameters for these RRATs can be seen in Table 2.1. We note that sky position and spectral index are covariant when the uncertainties on the former are large; however the uncertainty on the positions of these three sources is such that this covariance is broken (see Table 2.1).

For these three RRATs, the predicted diffractive scintillation timescales, $\Delta t_{\mathrm{d}}$, and bandwidths, $\Delta \nu_{\mathrm{d}}$, as well as the scattering timescale $\tau_{\mathrm{d}}$, have been calculated using the NE2001 model described in Cordes \& Lazio (2002), assuming a source velocity of $100 \mathrm{~km} \mathrm{~s}^{-1}$ at a center frequency of $1390 \mathrm{MHz}$. Additionally, for the NE2001 model we have calculated $\Delta \tau_{\mathrm{d}}$, the difference in $\tau_{\mathrm{d}}$ between the top and the bottom of the band. This allows us to see how much the pulse width changes between the top and bottom of the band due to scattering and check that our assumption that the pulse is the same width in each subband holds. These values are reported in Table 2.2 .

While the YMW16 electron density model (Yao et al., 2017) does not explicitly 
estimate scintillation parameters, one can estimate the scintillation bandwidth from

$$
2 \pi \Delta \nu_{\mathrm{d}} \tau_{\mathrm{d}}=C_{1}
$$

where $\tau_{\mathrm{d}}$ is the scattering timescale returned from the YMW16 model at the center frequency of the band estimated using the DM scaling equations from Bhat et al. (2004). We take $C_{1}=1$ for a thin scattering screen. We then estimate the scintillation timescale using

$$
\Delta t_{\mathrm{d}}=A_{\mathrm{ISS}} \frac{\sqrt{D \Delta \nu_{\mathrm{d}}}}{V_{\mathrm{ISS}} \nu}
$$

from Cordes \& Rickett (1998), where $D$ is the distance in kpc, $\Delta \nu_{\mathrm{d}}$ is in $\mathrm{MHz}, \nu$ is the observing frequency in $\mathrm{GHz}, V_{\text {ISS }}$ the velocity of the pulsar assumed to be $100 \mathrm{~km} \mathrm{~s}^{-1}$, and $A_{\mathrm{ISS}}=2.53 \times 10^{4} \mathrm{~km} \mathrm{~s}^{-1}$ for a uniform medium. The estimated values of $\Delta t_{\mathrm{d}}$ and $\Delta \nu_{\mathrm{d}}$ are reported in Table 2.2. Additionally we report the values of $\tau_{\mathrm{d}}$ but we do not report the change in scattering width, $\Delta \tau_{\mathrm{d}}$, across the band from the YMW16 model as $\tau_{\mathrm{d}}$ comes purely from the Bhat et al. (2004) scaling laws and is not modeled separately as in NE2001.

While $\Delta t_{\mathrm{d}}$ are on the same order as the period of these RRATs, $\Delta \nu_{\mathrm{d}}$ from both NE2001 and YMW16 are all < 1 MHz, so for subbands of $16 \mathrm{MHz}$ or greater, we are likely to average over many scintles and can therefore neglect the effects of scintillation in our analysis. Additionally, the difference in $\tau_{\mathrm{d}}$ between the top and bottom of our band, $\Delta \tau_{\mathrm{d}}$, is smaller than our time resolution, and confirms 
our assumption that the width of the pulse does not change substantially between subbands.

Specific to PSR J1819-1458 is its three-component profile. The components of this profile are known to vary by $\pm \sim 45 \mathrm{~ms}$ of the expected pulse phase (Lyne et al., 2009). Since we reference our TOAs to the brightest pulse in the observation and we do not know which part of the profile our detection is from, we use a TOA window of $6 \%$ of the expected pulse phase (instead of $5 \%$ ) in order to account for all pulses in the observation.

A sample of the single-pulses analyzed and their template fits can be see in Figure 2.3. While the sample shown is small compared to the total number of singlepulses detected, the wide variety we detect is apparent. While it can be seen that our template fitting may not fit components that appear to be real (e.g. pulses 1 or 7 ), this is due to the $>10 \%$ improvement in $\chi_{r}^{2}$ that we require to add a component, and prevents us from fitting noise into our pulse template as may occur in noisier pulses such as pulses 2 or 4 . The spectral index fits for these single-pulses are shown in Figure 2.4.

\subsubsection{Wait-time Analysis}

We use the same single-pulses found using the methods in $\S 2.3 .1$ in our waittime analysis; however, since subband sensitivity is not required, all pulses found at the $5 \sigma$ threshold are used. The wait-time for each pulse is determined by calculating the time between confirmed astrophysical pulses within an observation. The stan- 

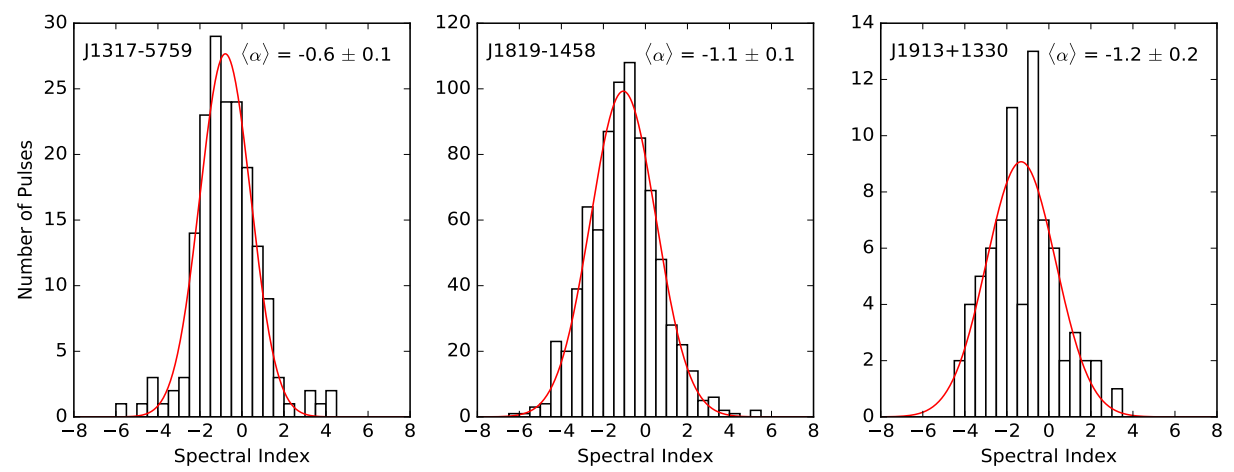

Figure 2.6 Distribution of single-pulse spectral indices for the RRATs analyzed in this work. The RRAT corresponding to each distribution is shown in the upper left corner, and the mean spectral index of each RRAT is reported in the upper right corner. The error reported on the spectral index is the error on the mean value.

dard Parkes observation length is $\sim 422$ pulse periods for J1819-1458, 1950 pulse periods for $\mathrm{J} 1913+1330$, and $\sim 681$ pulse periods for $\mathrm{J} 1317-5759$, with a few observations up to four times as long for each RRAT. Since we do not know how many pulses may have occurred between observations, no wait-time is reported between observations, so the longest possible wait-time is $\sim 2 \mathrm{hr}$.

In order to analyze the wait-time-flux-density relation for each RRAT, we bin the pulse wait-times by period and can then calculate the weighted mean flux density in each bin. We do not include any pulses where the error on the peak flux-density amplitude was larger than the calculated peak flux-density amplitude.

If the RRAT emission was described by a uniform distribution in time, we would expect the distribution of our pulse wait-times to be exponential. As a motivating example, the distribution of wait-time for the Parkes observations of PSR J1819-1458 is shown in Figure 2.5. We see that most pulses seem to be in groups, with the majority of pulses coming within a few periods of each other. 
There are also distinct tails to these distributions that suggest the distribution is not exponential.

We fit these distributions using the same least-squares fitting algorithm as in $§ 2.3 .1$ using four models. We first fit a pure exponential function and then fit more complex models to the wait-time distributions, including an exponential plus a Gaussian, an exponential plus a Maxwell-Boltzmann, and a log-normal plus a Gaussian. All models can be seen in Figure 2.5.

\subsubsection{Pulse Energy Analysis}

After calibrating each single-pulse found in $§ 2.3 .1$ using Eq. 2.1, we use the total flux density of each pulse, or the pulse energy, found using the same methods and filtering as in our wait-time analysis, to analyze the pulse energy distribution. The pulse energy is not dependent on bin size and is thus a more robust way of characterizing the emission mechanism than peak flux. A similar analysis on pulse amplitude distributions by Cui et al. (2017) found that most RRATs have a lognormal pulse amplitude distribution with little evidence of power-law tails, which describe GRP amplitude distributions (Mickaliger et al., 2012).

We use a similar method to Cui et al. (2017) to fit our pulse energy distributions for our three RRATs using three different models. We use a pure power-law model, a pure log-normal distribution, and finally a combined power-law and lognormal distribution. When fitting these models to our power-law distributions, we fit only from the pulse energy at which $N\left(S_{p}\right)$ is largest up to higher energies, as we 
cannot fit the energy distributions of weak pulses well with a log-normal, power-law, or combined model.

\subsection{Results and Discussion}

Our single-pulse analysis of the RRATs PSRs J1819-1458, J1913+1330, and J1317-5759 finds that the spectral indices of the single-pulses for all three RRATs follow a normal distribution. This is in line with work by both Kramer et al. (2003), who analyzed the single-pulse spectral indices of PSRs B0329+54 and B1133+16, and Karuppusamy et al. (2010), who analyzed Crab GRP spectra. Both studies found the single-pulse spectral index distributions well represented by a normal distribution.

Our analysis has also shown that the pulses do not seem to be emitted randomly, and we discuss the implications of this for RRAT emission mechanisms. Additionally we find that the time between pulses is not correlated with pulse flux density. Finally, we find that the distributions of the pulse energy for PSRs J1317-5759 and J1913+1330 are log-normal, while that of PSR J1819-1458 is log-normal with possible evidence of an additional power-law component.

\subsubsection{Spectral Indices}

In this work we have analyzed the spectral indices of single-pulses of RRATs for the first time. We find that, while there is a wide spread of spectral indices, they are well described by a Gaussian distribution. The peaks of the Gaussian 
Table 2.3 RRAT Mean Spectral Indices

\begin{tabular}{ccc}
\hline \hline Name & $\begin{array}{c}N_{8 \sigma} \\
\text { (pulses) }\end{array}$ & $\langle\alpha\rangle$ \\
\hline PSR J1819 - 1458 & 797 & $-1.1 \pm 0.1$ \\
PSR J1913 + 1330 & 75 & $-1.2 \pm 0.2$ \\
PSR J1317 - 5759 & 171 & $-0.6 \pm 0.1$ \\
\hline
\end{tabular}

Note. - Results of our spectral index analysis for the single-pulses of our RRATs. $N_{8 \sigma}$ is the number of single-pulses used in determining the mean spectral index, $\langle\alpha\rangle$, and thus were detected at a threshold of $8 \sigma$. We report the mean spectral index with $1 \sigma$ errors.

distributions agree within $1 \sigma$ with both the weighted and unweighted mean spectral indices for each respective RRAT. The distributions for all the three RRATs can be seen in Figure 2.6. We also report the mean spectral index and the number of single-pulses found for each RRAT in Table 2.3.

We note that there is a large amount of pulse-to-pulse variability in the measured single-pulse spectral indices shown in Figure 2.6, with a difference of $\sim 10$ between the highest and lowest values we find. There are very few other singlepulse spectral index studies in the literature for a comparison of our results. One single-pulse study of two pulsars, PSRs B0329+54 and B1133+16, by Kramer et al. (2003) found that the difference between the minimum and maximum spectral indices was $\sim 3$. However they also found that the individual components of the single-pulses spanned a slight larger range of spectral indices of $\sim 4$.

Additionally, a study by Karuppusamy et al. (2010) looked at the GRP spectral indices (both the main pulse and the interpulse) of the Crab pulsar. They fount that the distribution for both is also Gaussian and spans a range of spectral indices of about -10 to +10 . This is much larger than the range of spectral indices we find 
for PSRs J1819-1458, J1317-5759, and J1913+1330, but similar to that found for FRB 121102, which ranges from -10 to +14 . The range of our RRAT spectral indices falls in between those found by Kramer et al. (2003) and Karuppusamy et al. (2010), but with such a dearth of single-pulse spectral index studies, we cannot say whether the range we find is unusual.

Many studies have looked at the distributions of pulsar spectral indices (e.g. Lorimer et al., 1995; Maron et al., 2000; Bates et al., 2013; Jankowski et al., 2018). The mean value of pulsar spectral indices has varied slightly with each analysis. Lorimer et al. (1995) reported a mean spectral index of -1.6 in a study of 280 pulsars; Maron et al. (2000) reported a mean of $-1.8 \pm 0.2$ in a study of 281 pulsars, where the uncertainty is the error on the mean from individual spectral index measurements. Bates et al. (2013) reported a mean of -1.4 using Monte Carlo simulations, and Jankowski et al. (2018) reporting a weighted mean of $-1.60 \pm 0.03$ in a study of 441 pulsars where the uncertainty is the error on the weighted mean from individual spectral index measurements.

While individually each RRAT falls within the standard spread of mean pulsar spectral indices, which can range from steeper than -3 to flatter than -1 (e.g., see Lorimer et al., 1995; Jankowski et al., 2018), our mean RRAT spectral index is $-1.1 \pm 0.2$ and so is $2 \sigma$ or more away from most previous values. While the methods of our spectral index calculations are different than those traditionally used (e.g. Lorimer et al., 1995; Jankowski et al., 2018), all three RRATs exhibit flatter spectra at $1400 \mathrm{MHz}$ than might be expected from these previous pulsar spectral index studies, but are similar to magnetar spectral indices (e.g. Camilo et al., 2008; 
Pennucci et al., 2015).

We also note that in no single pulse do we see the same narrow-band frequency structure that is seen in FRB 121102 (Spitler et al., 2016; Gajjar et al., 2018; Michilli et al., 2018). As the majority of pulses analyzed for all three RRATs tend toward having negative spectral indices distributed normally around the mean, standard pulsar emission mechanisms seem unlikely to be the source of FRB 121102. Neither the frequency structure of the pulses nor the spectral index values of the pulses exhibit the characteristics of FRB 121102.

Another interesting comparison is with giant radio pulses (GRPs) from the Crab pulsar. Many multi-frequency studies of the Crab pulsar have found that the spectral index is relatively steep and varies widely. As mentioned before, Karuppusamy et al. (2010) find the single-pulse spectral index spread of the main component of the Crab pulsar's GRPs to range from about -10 to +10 . Other studies such as Popov \& Stappers (2007) found that the spectral index of the main component of the Crab pulsar's GRPs ranges from $\alpha=-1.7$ to -3.2 depending on the width of the pulse. In a wide-band study of Crab GRPs, Mikami et al. (2016) found that the spectral index of GRPs ranges from -1 to -4 , which is in line with the spread of our spectral index distribution, but generally steeper than the mean spectral indices of our RRATs. Similarly, Meyers et al. (2017) found that at low frequencies, between 120 and $165 \mathrm{MHz}$, the Crab GRPs show a spectral flattening with $\alpha=-0.7 \pm 1.4$, but between 732 and $3100 \mathrm{MHz} \alpha=-2.6 \pm 0.5$. This is steeper than the mean spectral index values for our three RRATs at $1400 \mathrm{MHz}$ and suggests that the RRAT pulses, although bright and sporadic, have emission mechanisms similar to normal 
pulsars.

\subsubsection{Wait Times}

\subsubsection{Wait-time Distribution Analysis}

We first analyzed the wait-time distributions, seen in Figures 2.5, 2.7, 2.8, and 2.9. If the emission was purely random, then the distribution would follow a pure exponential. We have attempted to model this dual population as a pure exponential, an exponential plus a Gaussian, an exponential plus a Maxwell-Boltzmann distribution, and as a log-normal plus a Gaussian. These processes are described in $\S 2.3 .4$. In the bottom panels, we have fit only the portion of the distribution shown.

We find that for all three RRATs, most pulses are emitted within a few periods of each other, as expected for a random distribution. For PSR J1317-5759 and the Parkes observations of PSR J1819-1458, there is a secondary component around wait-times of $\sim 25$ periods $(\sim 106$ s for PSR J1819-1458 and $\sim 66$ s for PSR J1317-5759). The increase in number of pulses around 25 periods, and the extended tails of longer wait-times for PSR J1317-5759 and the Parkes observations of PSR J1819-1458, are clearly not exponential. While these extended tails are reminiscent of a Weibull distribution, similar to a Poisson distribution but with an extra shape parameter (Oppermann et al., 2018), this cannot describe both the peak of pulses within a few periods and the secondary component observed in the wait-time distributions, and we do not fit for it.

For PSR J1913+1330, the wait-time distribution does not have the same in- 

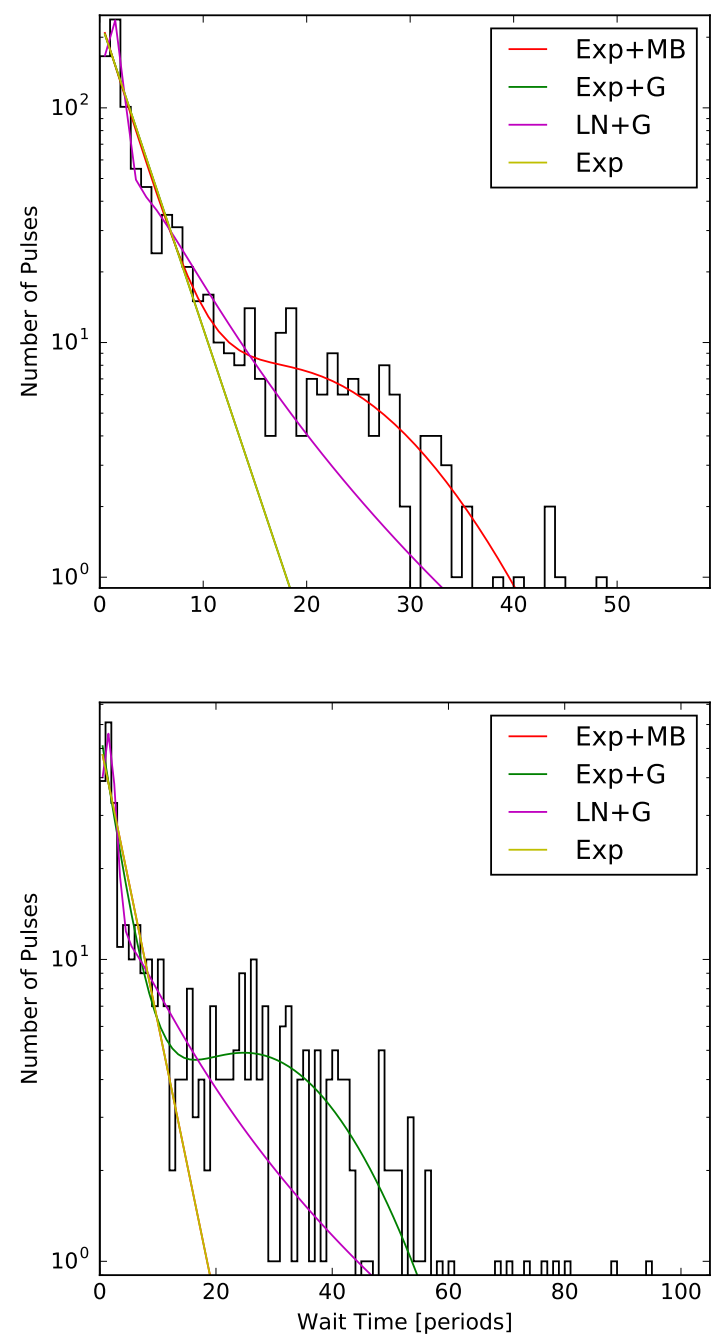

Figure 2.7 Upper panel: distributions of single-pulse wait times for the GBT observations of PSR J1819-1458 at S-band. We see that most pulses arrive within a few periods of each other. We also see that there is an extended tail of longer wait-times, but it does not go as far as that in Figure 2.5. The bump around 25 periods is less pronounced here than as seen in Figure 2.5. We fit the full distribution with an exponential plus a Gaussian (Exp $+\mathrm{G}$; green), an exponential plus a Maxwell-Boltzmann distribution (Exp+MB; red), a log-normal plus a Gaussian (LN+G; magenta), and a pure exponential (Exp; yellow). Lower panel: same as the upper panel but we have used only pulses with an S/N of $>20 \sigma$, approximately the equivalent detection threshold of Parkes at the L-band. We see that we clearly recover the bump around 25 periods and can detect longer wait-times. 

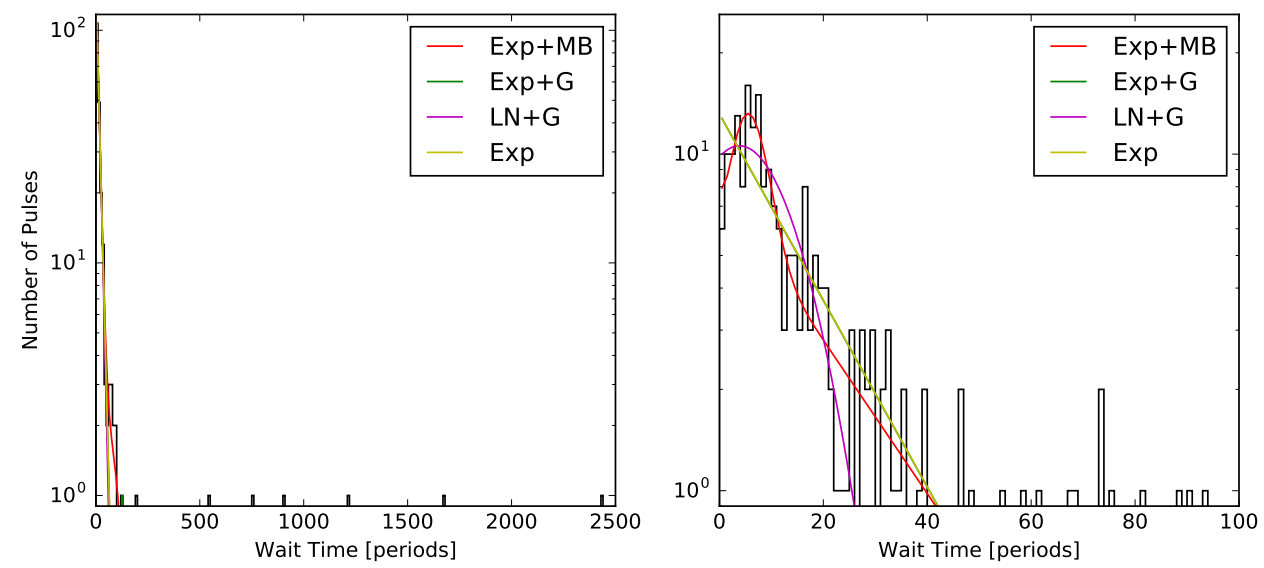

Figure 2.8 Left panel: distributions of single-pulse wait times for PSR J1913+1330 as observed with the Parkes Radio Telescope at the L-band. We have binned these pulses by 10 times their period and see that almost all pulses have shorter wait-times but there is an extended tail of long wait-times. We fit the full distribution with an exponential plus a Gaussian (Exp+G; green), an exponential plus a MaxwellBoltzmann distribution (Exp+MB; red), a log-normal plus a Gaussian ( $\mathrm{NN}+\mathrm{G}$; magenta), and a pure exponential (Exp; yellow). Right panel: same as the left panel but we have zoomed in on the first 100 periods and binned by the period. We see that most pulses appear within a few periods of each other.

crease in the number of pulses around wait-times of $\sim 25$ periods. However we note that PSR J1913+1330 has both the fewest number of detected pulses and the longest wait-times, extending out to almost the full length of the observation in some cases. Furthermore, we note that the GBT observations of PSR J1819-1458 do not show an extended tail of longer wait-times, and the increase in pulse number around 25 periods is much less pronounced.

A partial explanation of this secondary component can be found by considering the sensitivity of the GBT at the S-band to Parkes at the L-band. The GBT at $2 \mathrm{GHz}$ should be around four times more sensitive than Parkes at $1400 \mathrm{MHz}$. We can make a direct comparison between the two distributions if we only include single-pulses from the GBT observation that are bright enough have been detected 

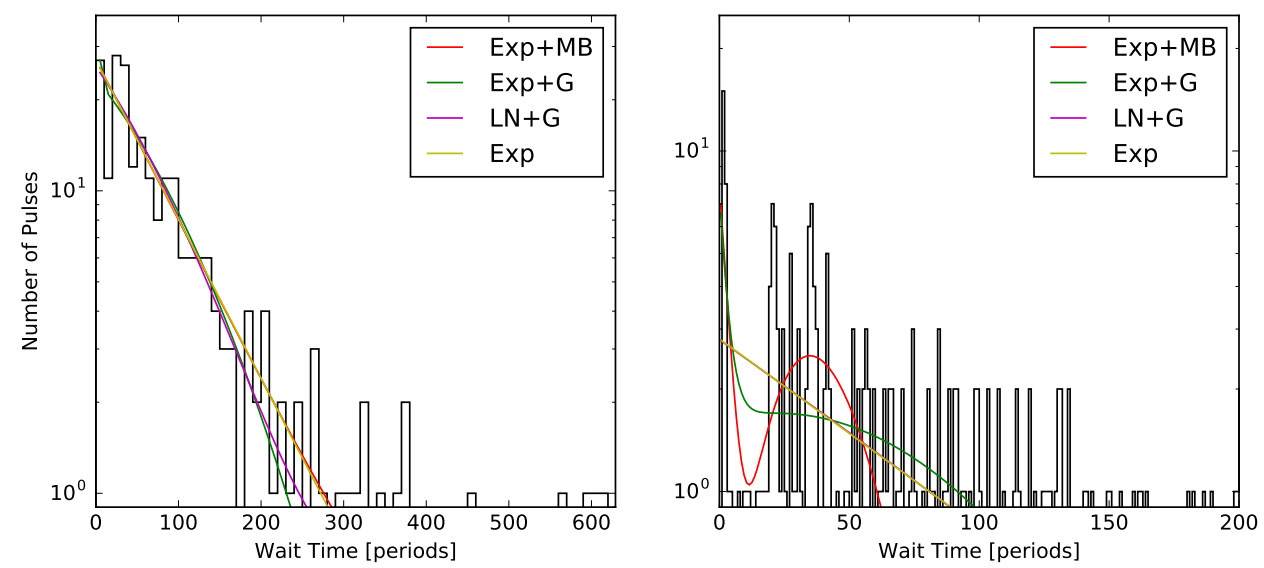

Figure 2.9 Left panel: distributions of single-pulse wait times for PSR J1317-5759 as observed by the Parkes Radio Telescope at the L-band. We have binned these pulses by 10 times their period and see that almost all pulses have shorter wait-times but there is a clear non-exponential tail of longer wait-times. We fit the full distribution with an exponential plus a Gaussian (Exp+G; green), an exponential plus a Maxwell-Boltzmann distribution (Exp+MB; red), a log-normal plus a Gaussian $(\mathrm{LN}+\mathrm{G}$; magenta), and a pure exponential (Exp; yellow). Right panel: same as the left panel but we have zoomed in on the first 200 periods and binned by the period. We see that most pulses appear within a few periods of each other but there is a second peak around 25 periods. 
Table 2.4 Wait-time Model Statistics

\begin{tabular}{ccccccc}
\hline \hline RRAT & Model & DoF & $\chi^{2}$ & $\chi_{r}^{2}$ & F-Stat & F-Sig \\
\hline PSR J1819-1458 (Parkes) & Exponential & 93 & 466 & 5.01 & - & - \\
& Exponential and Maxwell-Boltzmann & 91 & 181 & 1.99 & 70.8 & $1.1 \times 10^{-16}$ \\
& Exponential and Gaussian & 90 & 182 & 2.03 & 46.1 & $1.1 \times 10^{-16}$ \\
& Log Normal & 92 & 1070 & 11.63 & - & - \\
& Log Normal and Gaussian & 89 & 334 & 3.76 & 64.4 & $1.1 \times 10^{-16}$ \\
\hline PSR J1819-1458 (GBT) & Exponential & 62 & 181 & 2.91 & - & - \\
& Exponential and Maxwell-Boltzmann & 60 & 180 & 3.01 & - & - \\
& Exponential and Gaussian & 59 & 120 & 2.03 & 9.80 & $2.4 \times 10^{-5}$ \\
& Log Normal & 61 & 133 & 2.18 & - & - \\
& Log Normal and Gaussian & 58 & 80 & 1.38 & 12.58 & $1.9 \times 10^{-6}$ \\
\hline PSR J1317-5759 & Exponential & 128 & 112 & 0.87 & - & - \\
& Exponential and Maxwell-Boltzmann & 126 & 144 & 1.14 & - & - \\
& Exponential and Gaussian & 125 & 106 & 0.85 & 2.06 & 0.19 \\
& Log Normal & 127 & 121 & 0.95 & - & - \\
& Log Normal and Gaussian & 124 & 111 & 0.90 & 3.61 & $1.5 \times 10^{-2}$ \\
\hline PSR J1913+1330 & Exponential & 56 & 56 & 1.01 & - & - \\
& Exponential and Maxwell-Boltzmann & 54 & 39 & 0.72 & 12.17 & $4.3 \times 10^{-5}$ \\
& Exponential and Gaussian & 53 & 55 & 1.04 & 0.31 & 0.82
\end{tabular}

Note. - Model-fitting statistics for the wait-time distributions. DoF is degrees of freedom of the model, $\chi^{2}$ is the chi-squared value, and $\chi_{r}^{2}$ is the reduced chi-squared value. The F-statistic and significance correspond to the comparison of a more complex model with the less complex corresponding model. If there is no F-statistic reported, either the model is one of the base models, or the chi-squared value of the more complex model is larger than the base model. 
by Parkes at $1400 \mathrm{MHz}$. This filtered distribution is shown in the bottom panel of Figure 2.7 and shows the extended tail of wait-times, out to 100 periods, and recovers the slight bulge in number of pulses around 25 periods. Despite the lower number, this modulation of bright pulses around 25 periods is evidence that RRATs may exhibit short-timescale emission trends, contrary to Palliyaguru et al. (2011). Since we count wait-times between the pulses in a single observation, we do not see the long-timescale trends observed by Palliyaguru et al. (2011) as the the shortest trend they see in our three RRATs is for PSR J1317-5759 at $1.9 \mathrm{hr}$, longer than almost all of our individual observations.

Even accounting for this bias, for the full PSR J1819-1458 wait-time distribution, we see two populations of emission, one accounting for the pulses in each burst, described by the steeper part of the distribution with wait-times of a few periods, and another that accounts for the distribution of the bursts, described by a secondary distribution component "bump" around 25 periods. These distribution can also be seen in the wait-time distributions of PSR J1317-5759, shown in Figure 2.9 .

In addition to the $\chi_{r}^{2}$, we have also compared distributions using an F-test where appropriate. The F-statistic for model regression tells us if adding more parameters to our model makes a statistically significant contribution to the fit. The F-test assumes that the more complicated model has a smaller $\chi^{2}$ value, and is thus computed by

$$
F=\frac{\left(\chi_{1}^{2}-\chi_{2}^{2}\right) /\left(p_{2}-p_{1}\right)}{\left(\chi_{2}^{2}\right) /\left(n-p_{2}-1\right)}
$$



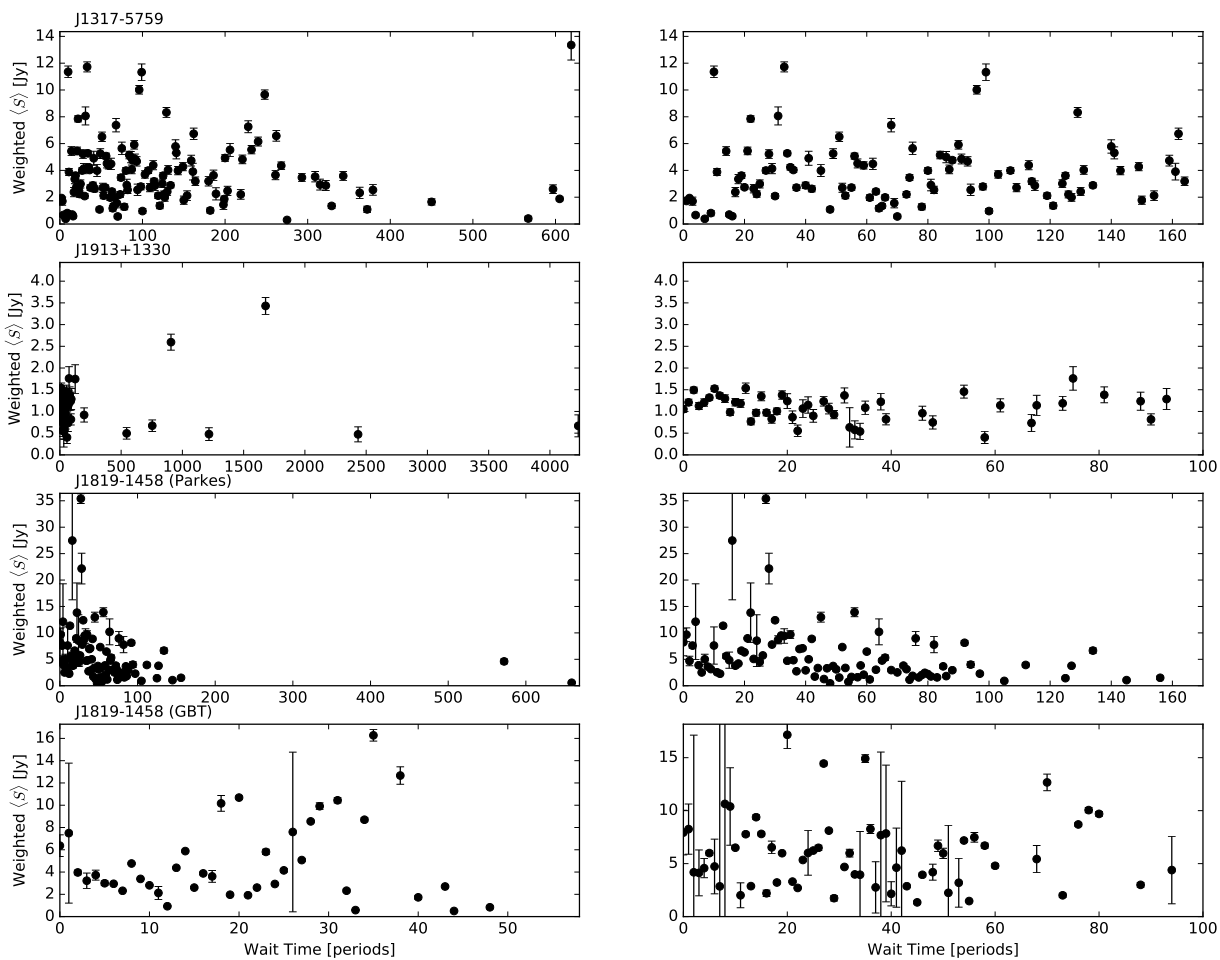

Figure 2.10 Weighted mean flux density of the single-pulses from each RRAT vs. wait time between pulses. The top three right panels are insets of the top three left panels. The right-hand bottom panel is not a inset but has the pulses that are below the approximate detection threshold of Parkes at the L-band filtered out. The RRAT corresponding to each set of panels is denoted in the upper left of the left-hand panels. We see that for all RRATs, the wait time does not correlate with flux density.

Here $\chi_{1 / 2}^{2}$ and $p_{1 / 2}$ are the chi-squared and number of free parameters that describe each model, where model 2 is the more complex model, and $n$ is the number of data points used to fit the models. If the $\chi^{2}$ of model 2 is greater than that of model 1 , we do not compute the F-statistic as this means the less complex model better fits the data.

We can determine the significance of the model from the Cumulative Density Function obtained using the scipy function fdtr. These values, along with the 

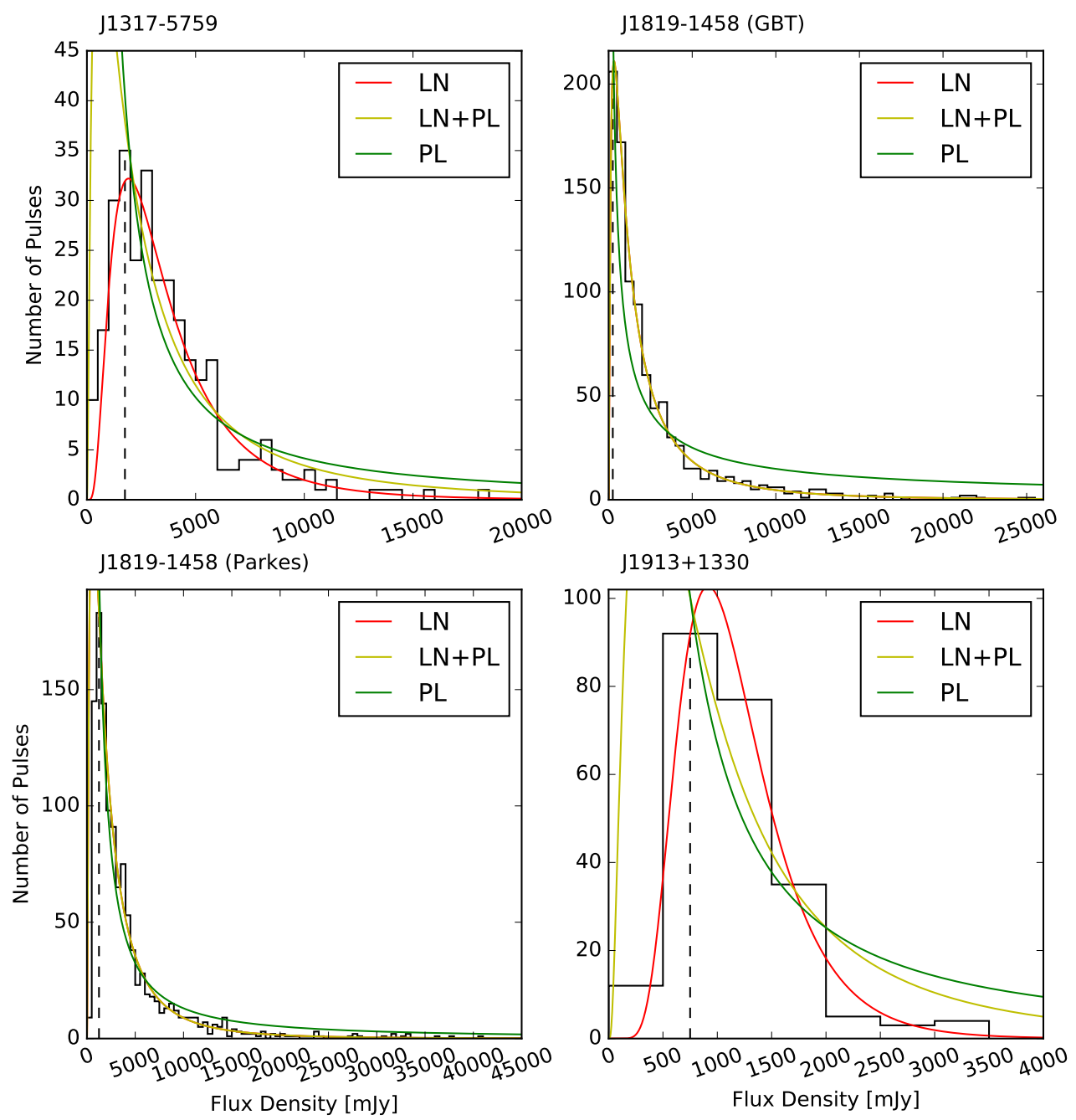

Figure 2.11 Distribution of single-pulse total flux density for each RRAT in our analysis. The RRAT corresponding to each distribution is denoted in above each plot at the upper left. Each distribution is fit with a log-normal (LN; red), power law (PL; green) or combination (LN+PL; yellow). We fit the distribution only to the dashed black line to reduce the influence of the non-detection of weak pulses. 
degrees of freedom $(\mathrm{DoF}), \chi^{2}$, and $\chi_{r}^{2}$ of each model, are reported in Table 2.4. We report these values only for the "zoomed-in" section of each wait-time distribution. Our models are unable to fit the extended wait-time tails of the full distributions well, showing that there could be some other, more complex emission process not well fit by our simple models.

From the statistics reported in Table 2.4, it is clear that none of our models has produced a good fit for PSR J1819-1458 or PSR J1317-5759. The Parkes observations of PSR J1819-1458 show that adding extra parameters to any of our models makes a significant improvement to our fits, but does not tell us if one is better than another. The GBT observations of PSR J1819-1458 show that adding a Gaussian to either a power law or log-normal is statistically significant, but again we cannot tell which model is preferred. The F-statistics for PSR J1317-5759 favor the two simplest models, and we cannot determine if an exponential better fits the wait-time distribution than a log-normal. This likely means that a more complex model is needed to fit these wait-time distributions. For PSR J1913+1330, we find that both a power law plus a Gaussian and a pure power law fit the distribution well, but adding the Gaussian is not statistically significant.

While the wait-time distribution of FRB 121102 is well described by a Weibull distribution (Oppermann et al., 2018), it is not as apt for the RRAT wait-time distributions. The latter shows the secondary peaks which are not seen in FRB 121102 and cannot be accounted for by the Weibull distribution although it can account for the extended tails of the long wait-times seen for the RRATs.

For PSR J1913+1330, an exponential distribution seems to best describe 
Table 2.5 Energy Model Statistics

\begin{tabular}{ccccccc}
\hline \hline RRAT & Model & DoF & $\chi^{2}$ & $\chi_{r}^{2}$ & F-Stat & F-Sig \\
\hline PSR J1819-1458 (Parkes) & Log normal & 51 & 43.3 & 0.85 & \multirow{2}{*}{$7.66 \times 10^{-3}$} & \multirow{2}{*}{0.99} \\
& Log normal and power law & 49 & 43.2 & 0.88 & 94.2 & $1.11 \times 10^{-16}$ \\
& Power law & 52 & 298 & 5.74 & & \\
\hline PSR J1819-1458 (GBT) & Log normal & 34 & 22.4 & 0.66 & $1.69 \times 10^{-5}$ & 0.99 \\
& Log normal and power law & 32 & 22.5 & 0.70 & 380 & $1.11 \times 10^{-16}$ \\
& Power law & 35 & 849 & 24.3 & & \\
\hline PSR J1317-5759 & Log normal & 22 & 20.5 & 0.93 & - & - \\
& Log normal and power law & 20 & 33.96 & 1.70 & 4.07 & $2.06 \times 10^{-2}$ \\
& Power law & 23 & 55.8 & 2.43 & & - \\
PSR J1913+1330 & Log normal & 3 & 83.9 & 2.80 & - & - \\
& Log normal and power law & 1 & 87.9 & 87.9 & - & \\
& Power law & 4 & 141 & 35.2 & & \\
& &
\end{tabular}

Note. - Model-fitting statistics for the RRAT energy distributions. DoF is degrees of freedom of the model, $\chi^{2}$ is the chi-squared value, and $\chi_{r}^{2}$ is the reduced chi-squared value. The top F-statistic is a comparison of the power law and the combined power law and log-normal fit. The bottom value is for the log-normal and the combined power law and log-normal fit. F-significance is the significance of adding the fit parameters for a more complex fit.

the wait-time distribution, which supports a random emission model. As we do not see a secondary component in the wait-time distribution of PSR J1913+1330, this seems reasonable. However it is likely that, as with PSR J1819-1458 and PSR J1317-5759, we are still missing some pulses. Weak pulse analyses have been performed for RRATs (e.g. Cui et al., 2017; Jiang et al., 2017) and recent work by Bhattacharyya et al. (2018) has found that PSR J1913+1330 exhibits a weak mode emission followed by long periods where there is no detectable emission. A lack of bright pulses could explain why our pulse wait-time distribution appears to support purely random emission, and does not show the secondary component seen in PSR J1819-1458 and PSR J1317-5759. 


\subsubsection{Flux-density-Wait-time Correlation}

In addition we analyzed the total integrated flux density of each pulse as a function of wait-time for each RRAT, including both PSR J1819-1458 observations, which can be seen in Figure 2.10. For each RRAT we have binned the pulses by wait-time in units of the RATT period and computed the weighted mean of the total integrated pulse flux density per wait-time. We find that for the three RRATs analyzed there is no correlation between the wait-time and the flux density of the pulse. We therefore conclude that the emission is not a consequence of a process through which energy is "stored up," such as magnetic recombination in the pulsar magnetosphere (e.g. Lyutikov, 2002; Rutledge, 2006).

\subsubsection{Energy Distributions}

Finally we present the pulse energy distributions for PSR J1819-1458, PSR J1317-5759, and PSR J1913+1330, shown in Figure 2.11. The different observations of PSR J1819-1458 are denoted above their respective panels. We have included every pulse above a $5 \sigma$ detection limit, filtered as with our wait-time analysis above. We note that, while we examine the pulse energy distributions, we have also compared these results to the pulse amplitude distributions and find they are similar.

Cui et al. (2017) have shown that the RRAT pulse amplitude distribution can be described by either a log-normal, as with pulsars, or a log-normal plus a powerlaw component, as in the power-law distribution of GRPs (e.g. Mickaliger et al., 2012). However PSR J1819-1458, PSR J1913+1330, and PSR J1317-5759 were 
not included in that analysis. Previously, McLaughlin et al. (2006) found the pulse amplitude distributions for PSR J1819-1458 and PSR J1317-5759 to be flatter, with a power-law index of $\sim 1$, than those of GRPs that show a power-law index of $\sim 2-3$. This difference in power-law index shows that the RRAT emission mechanism is likely separate from the emission mechanism of GRPs.

All three RRATs analyzed appear to show a population of pulses in the Parkes observations that have larger than standard flux densities and could be characteristic of a GRP power-law distribution (e.g. Mickaliger et al., 2012). To determine whether these are true power-law tails, we have fit a log-normal, power law, and a combined log-normal and power law, to each distribution, shown in Figure 2.11. We also compute the F-statistic for these distributions where appropriate as described in §2.4.2. We report these values as well as the DoF, $\chi^{2}$, and $\chi_{r}^{2}$ values for these models for each RRAT in Table 2.5.

For both observations of PSR J1819-1458, we find that a log-normal best describes the pulse energy distribution, and that adding a power-law component does not improve the fit significantly. For both PSRs J1317-5759 and J1913+1330 the pulse energy distribution is best described by a log-normal distribution.

Recent work by Mickaliger et al. (2018) found that the pulse energy distribution for RRATs showed both low- and high-energy peaks, indicative of the RRAT bursting, that could be fit with two log-normal distributions. We do not see evidence for this in our pulse energy analysis; however our analysis spans many epochs whereas Mickaliger et al. (2018) analyzed only data taken by the PMPS survey, so it is possible that we have bridged this gap with a larger distribution of pulses. 
While it is possible that the emission mechanism for RRATs is similar to that of GRPs, we lack the detections to fit the expected power-law tail. However as the spectral index distribution discussed in $§ 2.4 .1$ also does not follow the expected values for GRPs, we conclude that RRAT emission is not consistent with GRP emission.

We see from the significance of the F-test in Table 2.5 that the pulse energy distributions for PSR J1819-1458 in both the Parkes and GBT data are best described by a log-normal distribution. As expected with a mean spectral index of $-1.1 \pm 0.2$, where the uncertainty is from the mean of individual RRAT weighted means, the flux densities for the GBT pulses are weaker than those of the Parkes $1400 \mathrm{MHz}$ distribution. However the sensitivity of the GBT at $2 \mathrm{GHz}$ is greater than that of Parkes at $1400 \mathrm{MHz}$, which explains the larger number of pulses detected, and is evidence that the pulses we detect with Parkes are part of a broader distribution of pulses.

\subsection{Conclusions}

In this work we completed a single-pulse analysis of three RRATs, PSRs J1819-1458, J1317-5759, and J1913+1330, based on over 11 years of timing observations from the Parkes Radio Telescope, as well as archival data from the PMPS and an additional 7.5 hr observation of PSR J1819-1458 from the GBT. We have developed a method for determining the spectral indices of bright single-pulses and have shown them to be normally distributed around a mean spectral index that is comparable 
to the spectral indices of most pulsars. While there are few other single-pulse spectral index distributions, we find that our distribution is wider than those found by Kramer et al. (2003), and narrower than those found by Karuppusamy et al. (2010), although all are normally distributed.

We have shown that the pulsed emission is not uniformly distributed on small timescales, and even exhibits clustering around $\sim 25$ pulse periods in PSRs J1819-1458 and J1317-5759. Additionally, for PSR J1913+1330 we note that the extended wait-time tail is real; however we are unable to explain it. This is likely due to the intrinsic nature of the RRAT, similar to the processes in nulling pulsars (e.g. Wang et al., 2007). External mechanisms, such as an asteroid belt around the RRAT (Cordes \& Shannon, 2008), cannot be responsible for modulation on such short timescales, but can have an effect on longer timescales as discussed in Palliyaguru et al. (2011). For these three RRATs we have additionally shown that the time between the pulses is not correlated with the flux density of the pulse, thus the emission is not due to "storing up" energy.

Additionally we have analyzed the pulse energy distribution of our three RRATs. We found that PSRs J1317-5759 and J1913+1330 agree well with previous pulse energy and amplitude distribution studies done on other RRATs from Cui et al. (2017), showing a log-normal pulsar-like distribution. For PSR J1819-1458, we see that adding a power law to the log-normal distribution is not statistically significant. We have also found that these three RRATs do not exhibit a power-law tail in the pulse energy distributions, indicative of GRPs.

Neither the single-pulse narrow-band emission seen in FRB 121102, nor the 
narrowband frequency structure seen in FRB 170827 (Farah et al., 2018) are seen in the RRAT pulses, which suggests that the emission is different from RRAT emission. Instead our RRAT pulses seem to be broadband, more like non-repeating $\mathrm{FRBs}^{5}$. Although the spread of RRAT single-pulse spectral indices seems to be similar to the spread of spectral indices from FRB 121102, without more pulsar or RRAT single-pulse spectral index analyses, it is difficult to determine if this spread hints at a common emission process or not.

Unfortunately, the number of RRATs used in this analysis is small due to the fact that many bright pulses are necessary for a robust statistical analysis. Further observations of these RRATs at other frequencies would allow us to see if they exhibit spectra that change with frequency as has been found with the Crab pulsar. Observations with more sensitive telescopes would allow us to detect enough single-pulses to perform a single-pulse spectral analysis for other RRATs. Further observations of other bright RRATs would also allow us to perform our analysis on a larger sample to further explore their spectral distribution.

\footnotetext{
${ }^{5}$ see http://frbcat.org/ for a full list (Petroff et al., 2016).
} 


\section{Chapter 3}

\section{Analysis of Multi-hour Continuous Observations of Seven Millisecond Pulsars}

\subsection{Introduction}

Analyses of pulse times of arrival (TOAs) from pulsars, or pulsar timing, can be used to study many astrophysical phenomena. Not only can the objects themselves be studied, constraining their masses and equations of state (e.g., Antoniadis et al., 2013; Stovall et al., 2018; Cromartie et al., 2020), they can also be used as laboratories to probe extreme limits of general relativity (e.g., Kramer et al., 2006; Archibald et al., 2018; Zhu et al., 2019). In addition, groups like the North American Nanohertz Observatory for Gravitational Waves (NANOGrav; McLaughlin, 2013), the European Pulsar timing array (EPTA; Kramer \& Champion, 2013), and the Parkes Pulsar Timing Array (PPTA; Hobbs, 2013) use pulsar timing arrays (PTAs) made up of millisecond pulsars (MSPs) to search for gravitational waves from supermassive black hole binaries (e.g. Shannon et al., 2013; Zhu et al., 2014; Lentati et al., 2015; Shannon et al., 2015; Arzoumanian et al., 2016; Babak et al., 2016; Verbiest et al., 2016; Arzoumanian et al., 2018a; Aggarwal et al., 2019). In particular cases, 
continuous long observations such as those done by Dolch et al. (2014) can be used to produce single source gravitational wave limits (Dolch et al., 2016). Pulsar timing can also be used to study the properties of the interstellar medium (ISM) and how they change on timescales from hours to years (e.g., Coles et al., 2015; Levin et al., 2016; Lam et al., 2016b; Jones et al., 2017).

For astrophysics that requires extremely precise pulsar timing, such as the detection of gravitational waves, every source of noise must be well modeled (e.g. Lam et al., 2018a). As pulses propagate through the ISM, they are subjected to dispersion, scattering, and interstellar scintillation (Rickett, 1977) which can each be a source of noise (Shannon \& Cordes, 2012; Lam et al., 2016a, 2019) in the data. Interstellar scintillation is not correlated between observing epochs and thus will present as white (uncorrelated in time) noise. Changes in the pulse shape with frequency (Kramer et al., 1998; Pennucci et al., 2014) along with interstellar scintillation are another source of white noise. Variations in pulse phases and amplitudes, or pulse jitter, (Shannon \& Cordes, 2010, 2012; Lam et al., 2019) also appear as white noise. Dispersion and scattering are sources of red (correlated in time) noise, and stochastic variations in the pulse spin rate also manifest as red noise (Cordes, 1986; Cordes \& Rickett, 1998; Lam et al., 2016a).

The first source of TOA error we consider is from additive noise that causes template-fitting errors. Time-averaged pulse templates are cross-correlated with the observed pulses to determine the TOA. For an observed pulse averaged over some number of pulses, $N_{p}$, the precision of the TOA, or template-fitting error, goes as $1 / \sqrt{N_{p}}$ (Taylor, 1992; Dolch et al., 2014). However, if the pulse shape varies or has 
a low signal-to-noise ratio $(\mathrm{S} / \mathrm{N})$, then there may be additional errors above what is expected due to additive noise alone (Arzoumanian et al., 2015).

Pulse jitter is a second source of TOA error and occurs due to motions of coherent emission regions in pulsar magnetospheres (Cordes \& Shannon, 2010). While average pulse profiles are highly stable in time (e.g. Brook et al., 2018), for single pulses the phase and amplitude can be highly variable. As single pulses from MSPs are generally very weak, the pulse jitter is often difficult to measure directly from single pulses.

Diffractive interstellar scintillation (DISS), in combination with the fact that the pulse shape changes with observing frequency, cause a third source of TOA error (Liu et al., 2014; Pennucci et al., 2014). Diffractive scintillation due to the ISM causes constructive interference between the pulse ray paths which leads to increases in pulse intensity (and thus S/N) over particular frequencies, or "scintles" (Cordes, 1986; Cordes \& Rickett, 1998). These variations in the pulse S/N with the pulse shape changes in frequency may change the shape of the frequency-averaged pulse profile which would cause template-fitting errors.

DISS can also cause the pulses to be broadened, inducing additional timevariable delays (Hemberger \& Stinebring, 2008). However, for a turbulent (Kolmogorov) medium the average frequency scale of the interference, or scintillation bandwidth $\left(\Delta \nu_{\mathrm{d}}\right)$, and the average duration, or scintillation timescale $\left(\Delta t_{\mathrm{d}}\right)$, can be measured. Given a set of assumptions about the ISM, such as a homogeneous turbulent medium and spatial scale of the diffraction pattern larger than the observing baseline, the scintillation bandwidth can inform the magnitude of scattering and 
constrain the impact on pulsar timing (Cordes, 1986; Levin et al., 2016; Lam et al., 2016b; Lentati et al., 2017). However, as there will be a finite number of scintles on the frequency-time plane, our ability to accurately measure $\Delta \nu_{\mathrm{d}}$ and $\Delta t_{\mathrm{d}}$ is limited by the number of observed scintles (Cordes et al., 1990). This will also cause the scatter broadening function to be stochastic in what is known as the "finite scintle effect."

Finally, time delays due to dispersion are $\propto \mathrm{DM} \times \nu^{-2}$, where the dispersion measure (DM) is the integrated column density of free electrons along the line of sight and $\nu$ is the frequency of the radio emission. Since the ISM is a turbulent medium the DM can change with time and, if unmodeled, it can be a source of chromatic red noise (Keith et al., 2013; Jones et al., 2017).

Large scale studies of pulse jitter, scintillation parameters, and subsequent timing errors have been done by Lam et al. (2016a) and Levin et al. (2016) respectively on the NANOGrav 9-yr data set (Arzoumanian et al., 2015) and an updated analysis of the pulse jitter has also been completed for the NANOGrav 12.5-yr data set (Lam et al., 2019). However, the previous scintillation parameter measurements were limited by the typical $\sim 25$ minute observation lengths. While it is possible to characterize $\Delta \nu_{\mathrm{d}}$ on this timescale, $\Delta t_{\mathrm{d}}$ is almost always $\gtrsim 30$ minutes for these pulsars at the radio frequencies observed. This limits how accurately the DISS effects on pulsar timing can be estimated.

Additionally, Jones et al. (2017) measured the DM variations and evolution for 37 MSPs in the NANOGrav 9-yr data set to mitigate the chromatic red noise and found that the DM varies on timescales of days to years. However, due to the cadence 
of their observations, they were unable to probe variations on timescales shorter than $\sim 14$ days. Studies of DM variations on shorter timescales (e.g. Hankins et al., 2016) can additionally inform us about the ISM along a particular line of sight and its effects on precision pulsar timing. However, either higher cadence observations or longer observations are required to look for DM variations on these timescales.

Here we present our analyses of eight continuous multi-hour observations of seven MSPs, all part of the NANOGrav PTA. These observations allow us to study the scintillation parameters, pulse jitter, and DM variations on $\sim$ hour-long timescales (along each particular line of sight), similar to analysis done by Dolch et al. (2014) on a 24 hour multi-band continuous observation of PSR J1713+0747.

The standard NANOGrav timing procedure is to observe each MSP for typically $\sim 25$ minutes every few weeks (Arzoumanian et al., 2015, 2018c). Conversely, telescopes like the Canadian Hydrogen Intensity Mapping Experiment (CHIME), will make daily observations of multiple MSPs more common (Ng, 2018). However, each pulsar may only be visible to CHIME for $\sim 5$ minutes daily. The length of our observations present an opportunity to test the timing precision of a contiguous observation versus the same amount of time but split into several short observations (non-contiguous), where the pulse $\mathrm{S} / \mathrm{N}$ will be lower and the template-fitting error may increase beyond the expected $\propto 1 / \sqrt{N_{p}}$. This is useful in considering how adding CHIME pulsar timing data to NANOGrav will affect the timing precision.

We describe the observations and the basic data reduction pipeline in $§ 3.2$. Our methods for analyzing the pulse jitter, scintillation parameters, DM variations on short timescales, and timing precision of non-contiguous observations are described 
in $§ 3.3$. We present and discuss the results of our scintillation and ISM analysis in $\S 3.4$. The pulse jitter analyses are presented and discussed in $\S 3.5$. The results of our DM variations analysis are discussed in $§ 3.6$. Finally, we present the results of testing our timing precision with non-contiguous TOAs in time in $\S 3.7$. We offer concluding remarks in $§ 3.8$.

\subsection{Observations}

\subsubsection{Observational Data}

We observed seven NANOGrav MSPs (PSRs J0023+0923, J0340+4130, J0613-0200, J0645+5158, J1614-2230, J1832-0836, and J1909-3744) for between $~ 1.5$ and 〜6 hours each between MJDs 56724 (2014 March 8) and 56842 (2014 July 4). All observations were taken with the 100-m Green Bank Telescope (GBT) at the Green Bank Observatory. Each observation had a center frequency of $1500 \mathrm{MHz}$ and a bandwidth of $800 \mathrm{MHz}$ with $1.5625 \mathrm{MHz}$ frequency resolution. The raw profiles were folded in $\sim 15 \mathrm{~s}$ integrations in real time and coherently dedispersed at the DM listed in Table 3.1 by the GUPPI backend (DuPlain et al., 2008). Each pulse profile was divided into 2048 phase bins and recorded with two polarizations.

Over the course of the observations, instrumental difficulties caused parts of our frequency band to be lost in two of our observations, the first observation of PSR J0645+5158, and the observation of PSR J1909-3744. The PSR J0645+5158 observation was restarted and recalibrated, resulting in a $\sim 1$ hour gap between the two segments; the length of the sections are 1.5 and 2.5 hours, and were analyzed 
separately. Our observation of PSR J1909-3744 experienced similar data acquisition difficulties throughout the observation, resulting in the number of frequency channels being recorded dropping from 512 to 448, to 256, and finally to 192 . We did not analyze the 448 channel section of the observation in this work due to errors in the header of the data file. The other three sections of the observation were analyzed separately. The length of each observation can be found in Table 3.1.

\subsubsection{Data Reduction}

The flux and polarization calibration procedures as well as initial radio frequency interference (RFI) mitigation techniques closely follow those of Arzoumanian et al. (2018c). Our data reduction and analysis makes use of both the PsRCHIVE ${ }^{1}$ software package (Hotan et al., 2004; van Straten et al., 2012) and the PYTHON software package PYPULSE ${ }^{2}$ Lam (2017).

The polarization calibration observation was performed by injecting a broadband noise signal into both polarizations at the telescope before beginning the observation, and recording it with the GUPPI backend. We then calibrated both the phase angle between the polarizations and the differential gain with the noise signal with PSRCHIVE. Flux calibration was obtained from NANOGrav observations of the radio source $\mathrm{B} 1442+101$ taken at the closest date relative to the date of each of our MSP observations, taken with the same receiver. Full intensity profiles were obtained by summing the two polarizations of each profile together.

\footnotetext{
${ }^{1}$ http://psrchive.sourceforge.net/index.shtml

${ }^{2}$ https://github.com/mtlam/PyPulse
} 


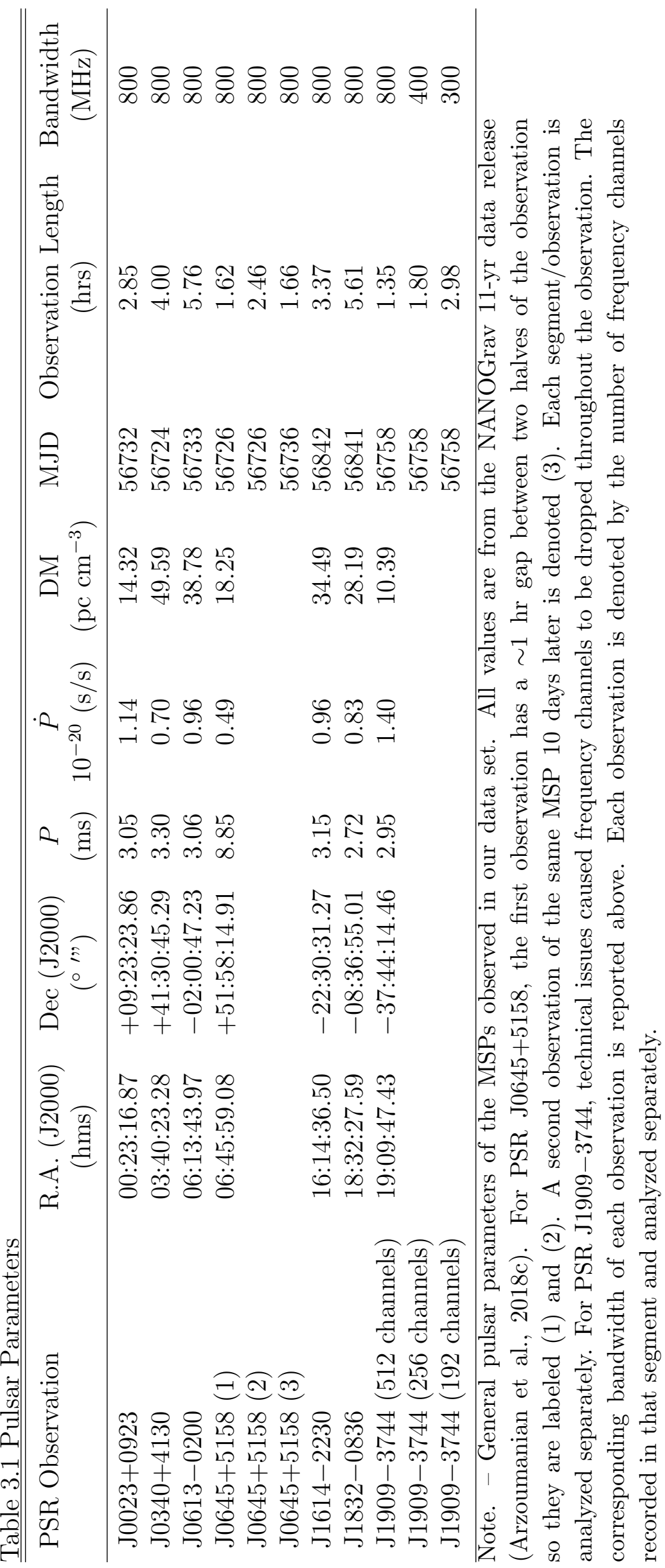


While a polarization calibration scan was done at the beginning of each of our observations and polarization cross-products were recorded, we only use total intensity measurements, obtained by summing the calibrated signals from pairs of orthogonal polarizations. This lack of calibration for feed coupling could produce time-variant profiles and TOAs at different parallactic angles (Liu et al., 2011). However, this is the same procedure as used for the NANOGrav 11-year dataset (Arzoumanian et al., 2018c) and there is no evidence of flux or profile variations due to incorrect polarization calibrations in those observations (Brook et al., 2018). We therefore expect excess noise to be minimal.

RFI mitigation was performed using the PSRCHIVE software. We first removed frequency channels known to be contaminated by RFI as denoted in the NANOGrav data reduction pipeline (Demorest et al., 2013). We then removed frequency channels and integrations where the off-pulse variance within a 20-channel/integration wide window was more than four times the median channel variance. Much of our data was heavily contaminated by RFI, so this off-pulse variance mitigation method was then rerun with a threshold of three times the median channel variance. Data were then checked manually to verify that RFI mitigation was successful, and any remaining RFI was manually removed.

For each particular analysis of the data, each observation was integrated in time and/or frequency using either the PSRCHIVE or PYPULSE packages to build up the $\mathrm{S} / \mathrm{N}$ and/or minimize computation time. The various subsections in $§ 3.3$ detail the subsequent data processing for each analysis. 


\subsection{Methods}

Here we lay out the methods for all analyses performed on our long observations. We first describe how we determined the total rms of our timing residuals and estimated the individual noise contributions. We discuss multiple ways to detect pulse jitter as in Shannon \& Cordes (2012); Shannon et al. (2014); Lam et al. (2016a, 2019). We then describe how the scintillation parameters, $\Delta \nu_{\mathrm{d}}$ and $\Delta t_{\mathrm{d}}$, were determined.

We also lay out methods for calculating pulsar secondary spectra to study the ISM along the line of sight as in Stinebring et al. (2000, 2019). We then detail our methods for constraining short timescale DM variations. Finally we describe how these long observations were used to assess the accuracy of non-contiguous pulsar TOAs when compared to TOAs generated from a contiguous time series.

\subsubsection{White Noise in Pulsar Timing Residuals}

White noise in pulsar timing residuals on short timescales is composed of three components: template-fitting errors, $\sigma_{\mathrm{S} / \mathrm{N}}$, which are dependent on the pulse $\mathrm{S} / \mathrm{N}$, DISS variations, $\sigma_{\text {DISS }}$, and errors due to intrinsic pulse jitter, $\sigma_{\mathrm{J}}$. We do not address errors due to calibration or residual RFI. The total white noise error contribution to our residuals, or their rms, $\sigma_{\mathcal{R}}$, can be characterized by

$$
\sigma_{\mathcal{R}}^{2}=\sigma_{\mathrm{S} / \mathrm{N}}^{2}+\sigma_{\mathrm{J}}^{2}+\sigma_{\mathrm{DISS}}^{2}
$$


For most MSPs $\sigma_{\mathrm{S} / \mathrm{N}}>\sigma_{\mathrm{J}} \gg \sigma_{\text {DISS }}$. However, in the high $\mathrm{S} / \mathrm{N}$ regime, we may observe $\sigma_{\mathrm{J}} \gtrsim \sigma_{\mathrm{S} / \mathrm{N}}$.

We used the pulsar timing packages PSRCHIVE and PYPULSE package to generate residuals and calculate $\sigma_{\mathcal{R}}$. For the duration of this work we followed the methods of Lam et al. (2016a) to generate "short-term" timing residuals, $\mathcal{R}(\nu, t)$, for each observation. We used the NANOGrav 11-yr timing parameters to fold our data. We assumed that these timing models were sufficiently accurate for our data sets so no model parameters were fit for.

However, since each epoch was analyzed separately, we determined only the pulse phase within an observation, or "initial timing residuals", $\delta t(\nu, t)$, and did not use the NANOGrav 11-yr timing parameters to determine the timing residuals. This method assumes that after using this timing model to fold the data we will be left with a polynomial expansion of pulse phase and spin period representative of the Earth-pulsar line of sight at the given epoch. These short-term residuals were calculated using the Fourier-domain estimation algorithm of Taylor (1992). Following Lam et al. (2016a), we then calculated the timing residuals, $\mathcal{R}(\nu, t)$, by fitting a polynomial over $\delta t(\nu, t)$ that included a constant offset for TOAs from each frequency channel and parabolic term common to all TOAs in time:

$$
\begin{gathered}
\delta t(\nu, t)=K(\nu)+a t+b t^{2}+n(\nu, t), \text { and } \\
\mathcal{R}(\nu, t) \equiv \hat{n}(\nu, t)=\delta t(\nu, t)-\left[\hat{K}(\nu)+\hat{a} t+\hat{b} t^{2}\right] .
\end{gathered}
$$


Here $a$ and $b$ are frequency-independent coefficients, $n(\nu, t)$ is additive white noise in frequency and time, including all components in Eq. 3.1, and $K(\nu)$ is a constant offset in frequency that accounts for frequency dependent variations such as profile evolution and scattering. The polynomial fit provides a simple way to remove deviations from the "true" timing model by use of the initial timing residuals. All values with carats are estimated quantities. Therefore Eqs. 3.2 and 3.3 denote $\mathcal{R}(\nu, t)$ as the estimated additive noise where frequency dependence between sub-bands has been subtracted off.

Determining $\sigma_{\mathcal{R}}$ as a function of integration time allowed us to both extrapolate the expected $\sigma_{\mathcal{R}}$ for a single pulse and check that $\sigma_{\mathcal{R}} \propto 1 / \sqrt{N}$, where now $N$ is the total integration time and is proportional to $N_{p}$. In particular we have used integration times of 15 and 30 seconds as well as 1, 2, 4, 8, 16, and 32 minutes all with $12.5 \mathrm{MHz}$ width per channel as used in the NANOGrav timing analysis (Arzoumanian et al., 2018c) resulting in 64 residuals per integration. We note again that the quadratic in Eq. 3.3 is not fit per channel and therefore there still remains a significant amount of white noise over the measurements from each of the 64 channels even when the mean of those is subtracted by estimating $\hat{K}$. Additionally, for longer integration times, the last integration was dropped if the length was not comparable to our desired time integration length. For our shortest observations with 32 minute integration times, we will have at minimum 128 residuals, so any variance that is absorbed using Eqs. 3.2 and 3.3 will be very small (Lam et al., 2016a).

Assuming that all pulses emitted by the pulsar are statically independent we were additionally able to test how well $\sigma_{\mathcal{R}}$ values follow the $1 / \sqrt{N}$ relationship by 
fitting not only for the value of $\sigma_{\mathcal{R}}$ at a integration time of a single pulse, but also for the slope as $1 / N^{\alpha}$. Any deviations from a slope of 0.5 would show that the pulses are not statistically independent (Helfand et al., 1975; Rathnasree \& Rankin, 1995).

Standard methods of pulsar timing assume the observed pulse is a scaled and shifted version of the pulse profile with added noise. For obtaining pulse TOAs using matched filtering, this assumption yields the minimum TOA error. Again following the formalism of Lam et al. (2016a), for a pulse with some effective width, $W_{\text {eff }}$, and $N_{\phi}$ phase bins, we write the template-fitting error (Cordes \& Shannon, 2010)

$$
\sigma_{\mathrm{S} / \mathrm{N}}=\frac{W_{\mathrm{eff}}}{S \sqrt{N_{\phi}}}
$$

where $S$ is the $\mathrm{S} / \mathrm{N}$ of the pulse taken as the peak to off-pulse rms ratio. Since the pulse $\mathrm{S} / \mathrm{N}$ is easily measured in this way, $\sigma_{\mathrm{S} / \mathrm{N}}$ is easily calculated.

Similarly, $W_{\text {eff }}$ of the pulse is dependent on both the pulse period, $P$, and the pulse template shape $U(\phi)$ as (Downs \& Reichley, 1983)

$$
W_{\text {eff }}=\frac{P}{N_{\phi}^{1 / 2}\left[\sum_{i=1}^{N_{\phi}-1}\left[U\left(\phi_{i}\right)-U\left(\phi_{i-1}\right)\right]^{2}\right]^{1 / 2}} .
$$

We note that $W_{\text {eff }}$ was calculated separately for each pulsar and can be determined separately for each frequency or backend. As all of our observations were taken at a central frequency of $1500 \mathrm{MHz}$ with the GUPPI backend, we used those parameters to determine $W_{\text {eff }}$ for all of the MSPs in our data set. However, both pulse jitter and scintillation can dynamically change the pulse profile which requires additional 
errors to be considered (Cordes \& Downs, 1985).

\subsubsection{Scintillation Parameters and $\sigma_{\text {DISS }}$}

As the ISM is dynamic, the frequency-dependent diffraction of the pulses due to the ISM will change as a function of time. This diffraction varies the path length of the pulses with time causing the pulse broadening function to change the pulse shape which is the source of $\sigma_{\text {DISS }}$ (Cordes et al., 1990; Lam et al., 2016a). While it is difficult to determine exactly how much the ISM is broadening the pulse due to covariances with frequency-dependent intrinsic pulse shape variations, the resulting scintillation pattern, or dynamic spectrum, has a characteristic timescale, $\Delta t_{\mathrm{d}}$, and frequency scale, $\Delta \nu_{\mathrm{d}}$. The scattering timescale, $\tau_{\mathrm{d}}$, is related to $\Delta \nu_{\mathrm{d}}$ by

$$
\tau_{\mathrm{d}}=\frac{C_{1}}{2 \pi \Delta \nu_{\mathrm{d}}}
$$

where $C_{1}$ is a coefficient ranging from $0.6-1.5$ depending on the geometry and spectrum of the electron density of the ISM (Lambert \& Rickett, 1999). Here we set $C_{1}=1$ as done in Levin et al. (2016). If $\Delta \nu_{\mathrm{d}}$ can be measured, the TOA error due to pulse scattering can be estimated directly as

$$
\sigma_{\mathrm{DISS}} \approx \frac{\tau_{\mathrm{d}}}{\sqrt{n_{\mathrm{ISS}}}}
$$

where $n_{\text {ISS }}$ is the number of scintles observed (Cordes \& Shannon, 2010). This determines our ability to accurately measure $\Delta \nu_{\mathrm{d}}, \Delta t_{\mathrm{d}}$, and thus $\sigma_{\text {DISS }}$. 
For Eq. 3.7 to be true, $n_{\mathrm{ISS}}$ must be large. If $\Delta t_{\mathrm{d}}$ and $\Delta \nu_{\mathrm{d}}$, can be measured, $n_{\mathrm{ISS}}$ for a single observation of length $T$ and total bandwidth $B$ can be estimated from

$$
n_{\mathrm{ISS}} \approx\left(1+\eta_{t} \frac{T}{\Delta t_{\mathrm{d}}}\right)\left(1+\eta_{\nu} \frac{B}{\Delta \nu_{\mathrm{d}}}\right)
$$

Here $\eta_{t}$ and $\eta_{\nu}$ are filling factors in the range 0.1-0.3 (Cordes \& Shannon, 2010), which were both set to 0.2 as in Levin et al. (2016).

For many NANOGrav observations, $\Delta t_{\mathrm{d}}>T$ and is not measurable, so $(1+$ $\left.\eta_{t}\left(T / \Delta t_{\mathrm{d}}\right)\right) \approx 1$. However, if $\Delta t_{\mathrm{d}}$ is more accurately measured, $n_{\mathrm{ISS}}$ and thus $\sigma_{\text {DISS }}$, can be more accurately estimated. After determining $\sigma_{\text {DISS }}$ Eq. 3.1 can then be solved for $\sigma_{\mathrm{J}}$.

\subsubsection{Measuring Scintillation Parameters}

As our observations ranged from $\sim 1.5-6$ hours in length and were taken, in most cases, over an $800 \mathrm{MHz}$ bandwidth, we expected $n_{\text {ISS }}$ would be large based on previous measurements from Levin et al. (2016) and estimates from the NE2001 electron density model (Cordes \& Lazio, 2002). We therefore attempted to measure $\Delta t_{\mathrm{d}}$ and $\Delta \nu_{\mathrm{d}}$ independently for each MSP. We did this using two methods.

In order to fit the scintillation parameters, after our initial data reduction we created a 2-D dynamic spectrum for each MSP using the PYPULSE PYTHON package. The dynamic spectrum shows how the intensity of the pulsar emission varies as a function of both time, $t$, and frequency, $\nu$. In PYPULSE this is performed by first subtracting baseline variations, and then taking a template profile, here the 

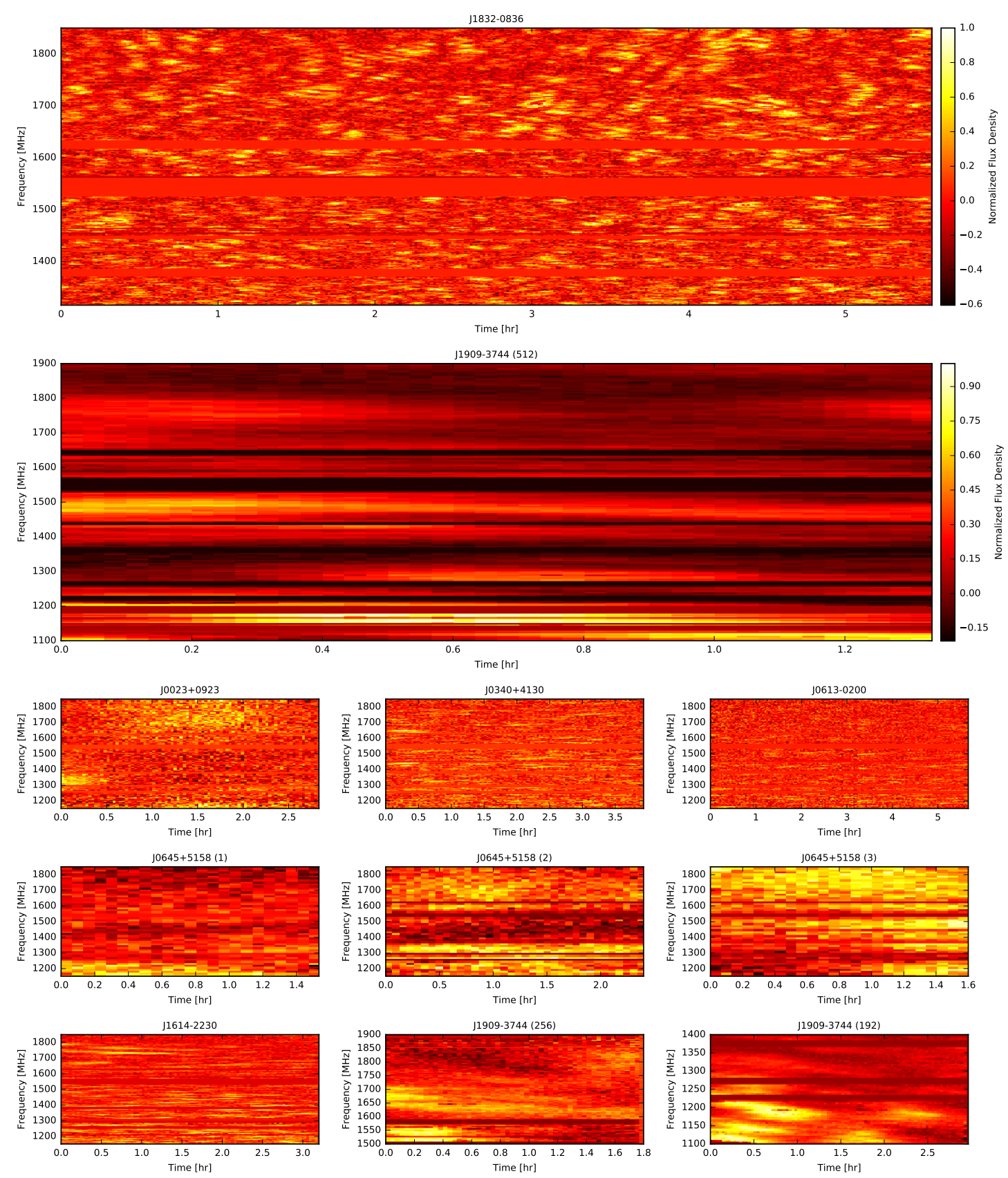

Figure 3.1 Unstretched dynamic spectrum for all MSPs analyzed in this work. Bright (yellow) patches show scintles. RFI zapped channels had their values replaced with the mean value of the dynamic spectrum. All values are reported in terms of normalized flux density. The two large dynamic spectra (PSRs J1832-0836 and J1909-3744) are shown to contrast narrow, short scintles with wide, long scintles. 
NANOGrav 11-yr templates, and using the template-matching procedure of Taylor (1992) to calculate the peak amplitude, or intensity, of the profile in each timefrequency bin in mJy. These intensity values are used as the dynamic spectrum values. Any frequency channels that were zapped due to RFI were replaced with the mean power value of the full dynamic spectrum.

While this peak-amplitude method differs from Levin et al. (2016), which subtracts the off-pulse flux from the on-pulse flux and divides by the mean off-pulse value, we still obtain robust dynamic spectra. Our data were polarization and flux calibrated and the baseline is subtracted so we can be confident that no variations in the baseline are included in our peak-amplitude dynamic spectra values. The pulse profiles of all MSPs in this work are sharply peaked, so the peak-amplitude returned by the template-matching procedure will be a robust proxy for the total on-pulse flux. Thus any variations in the peak-amplitude values should therefore be due to scintillation.

For some pulsars it is obvious that $\Delta t_{\mathrm{d}}$ and/or $\Delta \nu_{\mathrm{d}}$ are much larger than the initial integration time of 15 seconds or frequency channel size of $1.5625 \mathrm{MHz}$. In these cases, a coarser resolution in time and/or frequency was used to build up pulse $\mathrm{S} / \mathrm{N}$, and we integrated in time or frequency such that there were at least $\sim 5$ integrations/frequency channels spanning each scintle. The resolution of the dynamic spectrum for each MSP can be seen in Figure 3.1.

To measure the characteristic scales for both $\Delta t_{\mathrm{d}}$ and $\Delta \nu_{\mathrm{d}}$ over our band, we had to take into account the frequency dependence of both parameters. The frequency dependencies of these parameters differ however, with $\Delta t_{\mathrm{d}} \propto \nu^{1.2}$ (Rickett, 
$1977)$ and $\Delta \nu_{\mathrm{d}} \propto \nu^{4.4}$ (Cordes, 1986). With this relation, $\Delta t_{\mathrm{d}}$ will change by less than a factor of two over the bandwidth. In addition, $\Delta t_{\mathrm{d}}$ is often of order the length of the observation, whereas $\Delta \nu_{\mathrm{d}}$ is usually much smaller than our bandwidth, so we expect fewer scintles in time than in frequency.

However, the dependence of $\Delta \nu_{\mathrm{d}}$ on frequency is much steeper. To account for this, we adopted the same "stretching" method used by Levin et al. (2016) and stretched the dynamic spectrum to a reference frequency of $1500 \mathrm{MHz}$, assuming the $\nu^{4.4}$ frequency dependence.

We then computed the 2-D autocorrelation function (ACF) of each dynamic spectrum. An example can be seen in the left panel of Figure 3.2. The first method used to estimate the scintillation parameters is similar to that of Levin et al. (2016). We summed the 2-D ACF over a subsection of the time axis for $\Delta \nu_{\mathrm{d}}$, or over a subsection of the frequency axis for $\Delta t_{\mathrm{d}}$, such that the central power region (in either time or frequency) was summed over without adding in noise. A Gaussian, centered at zero-lag (in either time or frequency), was then fit to each resulting 1-D $\mathrm{ACF}$, as shown in the right panels of Figure 3.2 for example.

Often there is a noise spike centered at zero-lag in time and/or frequency which can bias the Gaussian fit. In order to minimize the effect of this spike, the value of the 1-D ACF at zero-lag was replaced with the average of the two points to either side of it. The value of $\Delta \nu_{\mathrm{d}}$ was taken to be the half-width at half max of the resulting Gaussian fit over the $1-\mathrm{D}$ ACF vs time lag, and $\Delta t_{\mathrm{d}}$ was the half-width at $e^{-1}$ of the Gaussian fit over the 1-D ACF vs frequency lag (Cordes, 1986).

The second method we used utilizes PYPULSE to fit a 2-D Gaussian to the 2-D 

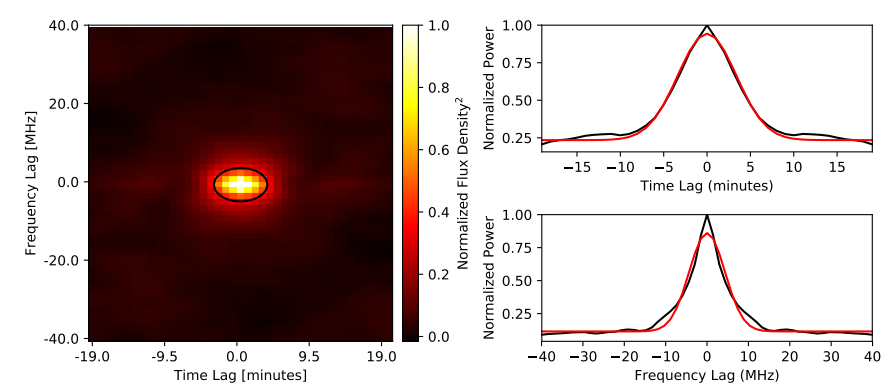

Figure 3.2 Left: 2-D Autocorrelation function (ACF) of the dynamic spectrum for PSR J1832-0836 after stretching. The black contour shows the 2-D Gaussian fit of the 2-D ACF. Upper Right: 2-D ACF summed along the frequency axis with the central noise spike removed. The red line is the Gaussian used to obtain the scintillation timescale. Lower Right: Same as above but summed along the time axis with the Gaussian used to describe the scintillation bandwidth.

ACF. The central noise spike described above was again replaced with the average of these two points. We used just the central subsection of the 2-D ACF such that the full central power region was included with as little noise as possible. An example of the 2-D fit is shown by the black contour in the left panel of Figure 3.2.

Since all of our observations are over an hour in length, if we were able to resolve $\Delta t_{\mathrm{d}}$, the 2 -D fitting allowed us to better constrain and inform the fit values for $\Delta \nu_{\mathrm{d}}$ and $\Delta t_{\mathrm{d}}$. Additionally, the long observation times allowed us to fit for the drift rate in the scintles to constrain refractive variations through the ISM as (Hewish, 1980; Cordes et al., 1986)

$$
\frac{d \nu}{d t}=\frac{\nu V_{\perp}}{2 d_{s} \theta_{r}}
$$

where $V_{\perp}$ is the pulsar's velocity perpendicular to the line of sight, $d_{s}$ is the distance between the Earth and a thin screen of material (in terms of the total distance $D$ and the pulsar-screen distance $D_{s}, d_{s}=D-D_{s}$ ), and $\theta_{r}$ is the component of the 
refractive angle along the direction of the pulsar's motion. This drift rate is fit for by a rotation of the $2-\mathrm{D}$ Gaussian fitted to the $2-\mathrm{D} \mathrm{ACF}$, in the frequency and time lags plane.

\subsubsection{Secondary Spectra}

The dynamic spectrum can also be used to study deviations from the typical thin screen model of scattering (Scheuer, 1968). The 2-D Fourier transform of the dynamic spectrum, or secondary spectrum, may show scitillation "arcs" which can be used to study the structure of a scattering screen between us and the pulsar (Stinebring et al., 2001; Cordes et al., 2006). The shape, thickness, and number of arcs can be used to infer the location of the screen between us and pulsar, the thickness of the screen on $\sim \mathrm{AU}$ scales, and the number of screens causing the scattering (Stinebring et al., 2001, 2019).

Only two MSPs have already been seen to exhibit scintillation arcs, PSRs J0437-4715 (Bhat et al., 2016; Reardon, 2018) and B1937+21 (Walker et al., 2013), so these observations present an opportunity to search for scintillation arcs in these MSPs for the first time. Secondary spectra are generated with PYPULSE by first taking a 2-D fourier transform of the dynamic spectrum, and then taking the square of the absolute magnitude of the transformed spectra (Stinebring et al., 2001; Reardon et al., 2019). As the power of the secondary spectra is log-normally distributed, we took the log of the secondary spectra to better search for scintillation arcs. 


\subsubsection{Maximum Likelihood Estimates of Pulse Jitter}

Instead of directly solving Eq. 3.1 using values obtained from Eqs. 3.4 and 3.7 to determine the pulse jitter, we can also follow the methods of Lam et al. (2016a). To estimate $\sigma_{\mathrm{J}}$ from the timing residuals, we can rewrite Eq. 3.1 as a function of the pulse S/N (Lam et al., 2016a),

$$
\sigma_{\mathcal{R}}^{2}(S)=\sigma_{\mathrm{S} / \mathrm{N}}^{2}(S)+\sigma_{\mathrm{DISS}}^{2}+\sigma_{\mathrm{J}}^{2}=\left(\frac{W_{\mathrm{eff}}}{S \sqrt{N_{\phi}}}\right)^{2}+\sigma_{C}^{2},
$$

where $\sigma_{C}^{2}=\sigma_{\text {DISS }}^{2}+\sigma_{\text {J }}^{2}$ is the rms that is "constant" in $S$ and $\sigma_{\mathcal{R}}^{2}(S)$ is calculated from the rms of $\mathcal{R}(\nu, t)$. If we can measure $\sigma_{C}^{2}$, we can estimate $\sigma_{\mathrm{J}}$ using $\sigma_{\text {DISS }}$ obtained from $\Delta t_{\mathrm{d}}$ and $\Delta \nu_{\mathrm{d}}$.

To estimate $\sigma_{C}^{2}$ we performed the maximum likelihood analysis over the residuals detailed in Lam et al. (2016a). This was done by assuming that, for a given $\mathrm{S} / \mathrm{N}$, our residuals will follow a Gaussian distribution described by

$$
f_{\mathcal{R} \mid S}\left(\mathcal{R} \mid S, \sigma_{C}\right)=\frac{1}{\sqrt{2 \pi \sigma_{\mathcal{R}}^{2}}} e^{-\mathcal{R}^{2} /\left(2 \sigma_{\mathcal{R}}^{2}\right)} .
$$

From this we could determine the PDF of $\sigma_{C}^{2}$ from our residuals and predetermination of $\sigma_{\mathrm{S} / \mathrm{N}}$. This PDF was evaluated with PYPULSE to determine the value of $\sigma_{C}^{2}$ as described in Lam et al. (2016a). An F-test with a significance value of $0.05(2 \sigma)$ was used to determine whether or not the determined value of $\sigma_{C}^{2}$ was significant. The $95 \%$ upper limit on $\sigma_{C}^{2}$ is reported if it was not.

While Lam et al. (2016a) used a S/N cutoff of $\sim 3$ to filter out low-significance 
noise from the data, we did not include a low $\mathrm{S} / \mathrm{N}$ cutoff as many of our MSPs have few, if any, TOAs above this threshold. To mitigate noise in our data due to our lack of a low $\mathrm{S} / \mathrm{N}$ cutoff, outlier residuals were removed via sigma stripping with a $3 \sigma$ threshold where $\sigma$ is determined from the overall distribution of $\sigma_{\mathcal{R}}$.

Since $\sigma$ is determined from the data itself, the choice of threshold used for the sigma stripping will impact the resulting noise measurements. The lower and more

spread out the TOA S/Ns are, the more the choice of threshold will impact the results, as significantly more TOAs will be excised at lower thresholds. Therefore careful testing of different thresholds should be taken. For most of our pulsars we found no statistically significant difference when using thresholds between $2.5 \sigma$ and $4 \sigma$, but smaller thresholds removed over half of the TOAs, which would artificially bias our results to smaller values of pulse jitter, since we can directly measure $\sigma_{\text {DISS }}$.

\subsubsection{Pulse Jitter Sub-Band Correlations}

Correlations between the frequency sub-banded residuals can also be indicative of pulse jitter (Shannon \& Cordes, 2012; Shannon et al., 2014; Lam et al., 2016a). If the size of the sub-bands is $\gtrsim \Delta \nu_{\mathrm{d}}$, then the timing residuals will not be correlated across our band due to DISS. However, if the TOAs have a low $\mathrm{S} / \mathrm{N}$, then $\sigma_{\mathrm{S} / \mathrm{N}} \gg$ $\sigma_{\mathrm{J}}$, and any correlations will be undetectable, despite $\sigma_{\mathrm{S} / \mathrm{N}}$ being uncorrelated across the band.

While the flux of the MSPs in our long observations are not unusual when compared to other observations of the same MSPs at the same frequencies (Lam 
et al., 2019), the steep spectral index that most pulsars exhibit (Jankowski et al., 2018) means that the $\mathrm{S} / \mathrm{N}$ of these TOAs is much lower than similar observations at lower frequencies. To try to increase the $\mathrm{S} / \mathrm{N}$ of the TOAs, we used sub-band widths of $400 \mathrm{MHz}$ and integration times of 16 minutes. Since we obtained measurements of $\Delta \nu_{\mathrm{d}}$ for this epoch, we can be confident that these sub-band sizes are large enough to mitigate DISS correlations. While this yielded a two sub-band correlation with a small number of TOAs, it also increased the $\mathrm{S} / \mathrm{N}$ of the TOAs used.

\subsubsection{Measuring DM Variations on Hour Timescales}

Delays due to dispersion by the ISM follow

$$
\Delta t \simeq 4.15 \times 10^{6} \mathrm{~ms} \times \mathrm{DM}\left(\frac{1}{1500^{2}}-\frac{1}{\nu^{2}}\right)
$$

where $\Delta t$ is in ms, DM is in $\mathrm{pc} \mathrm{cm}^{-3}, \nu$ is the frequency in $\mathrm{MHz}$, and the delay here is referenced to a frequency of $1500 \mathrm{MHz}$. Previously Jones et al. (2017) studied DM variations in NANOGrav MSPs, including those in our sample, on timescales of days to years. They found that four MSPs, including PSR J1614-2230, had DMs that varied on timescales less than 14 days. However, they were unable to probe variations on shorter timescales due to the cadence of their observations. However, individual NANOGrav observations could be used to study DM variations on timescales as short as $\sim 1-30$ minute over $800 \mathrm{MHz}$ bandwidth for the GBT or $600 \mathrm{MHz}$ bandwidth for Arecibo.

The nature of our long observations allows us to look for DM variations on 
hour-long timescales along multiple lines of sight. Since NANOGrav observations are typically $\sim 25$ minutes, we can obtain multiple DM measurements based on segments of equivalent length over the course of long observation, although over just a single frequency band. This allows us to look for DM variations in these MSPs on shorter timescales than have been studied before, but longer timescales than can be studied using the individual NANOGrav observations.

To do this, we split our observations into 32 minute integrations spanning 64 sub-bands (12.5 MHz per band), the same values used by NANOGrav (Arzoumanian et al., 2018c), and fit for the DM in 32 minute sections with the TEMPO ${ }^{3}$ pulsar timing package. The results are then visually inspected for apparent variations or evolution in time.

We can also estimate what the expected DM variations from the ISM for each MSP on the timescale of hours will be using the same method as in Cordes et al. (2016). In the strong scattering regime, the size of the scattering cone of the pulsar is much larger than the Fresnel scale,

$$
r_{F}=\sqrt{\frac{\lambda D}{2 \pi}} .
$$

Here $\lambda$ is the observing wavelength, here $1.5 \mathrm{GHz}$, and $D$ is the distance to the pulsar, given in Table 3.3, from the NANOGrav 11-yr parallax measurements. As the radio wave propagates through the ISM, the phase of the wave, $\phi$, is perturbed,

\footnotetext{
${ }^{3}$ HTTP://TEMPO.SOURCEFORGE.NET
} 
causing a change in DM as

$$
\delta \mathrm{DM}=\frac{d \phi / d \nu}{\lambda \mathrm{r}_{\mathrm{e}}}
$$

where $\mathrm{r}_{e}$ is the classical electron radius. with an observing frequency of $1.5 \mathrm{GHz}$, we can convert $\lambda \mathrm{r}_{e}$ in units of $\mathrm{pc} \mathrm{cm}{ }^{-3}$ to find $\delta \mathrm{DM}=5.75 \times 10^{-8} \mathrm{pc} \mathrm{cm}^{-3}$ per radian of phase perturbation.

We can then use transverse velocity of the pulsar and the length of the observation to determine how many $r_{F}$ lengths it travels, and thus how many multiples of $\delta \mathrm{DM}$ we would expect the DM to vary by over the course of the observation. Here the transverse velocity is derived either from the proper motions measured in the NANOGrav 11-yr timing parameters or from the measured scintillation parameters as described in Cordes \& Rickett (1998),

$$
V_{\mathrm{ISS}}=A_{\mathrm{ISS}} \frac{\sqrt{D \Delta \nu_{\mathrm{d}}}}{\nu \Delta t_{\mathrm{d}}},
$$

where $A_{\text {ISS }}=2.53 \times 10^{4} \mathrm{~km} \mathrm{~s}^{-1}$. Both transverse velocities are reported in Table 3.3. As stated in Cordes et al. (2016), for most pulsars, the expected timescale for DM variations due purely to the ISM is $\sim$ weeks, suggesting that we would not expected to see any DM variations over the course of our long observations. We report the expected $\delta \mathrm{DM}$ from the ISM over each of our individual MSP long observations in $\S 3.6$. 


\subsubsection{Timing Precision with Non-Contiguous TOAs}

Our observations also offer an opportunity to test the precision of pulsar timing residuals when using a set of TOAs contiguous in time versus non-contiguous TOAs representing the equivalent length of time. This comparison is particularly useful for considering the impact of CHIME on precision pulsar timing, as it will be able to time many pulsars daily, but only for $\sim 5$ minutes (Ng, 2018). Eq. 3.4 breaks down at very low S/N (Arzoumanian et al., 2015), which may be the case for some pulsars in this mode of observing. For PTAs such as NANOGrav, increasing the cadence of pulsar observations will increase sensitivity to continuous wave sources and tracking rapid DM variations, but will still require high timing precision (Lam, 2018).

To test this precision, we folded each of our long observations into four minute integrations with 64 frequency channels $(12.5 \mathrm{MHz}$ per channel). We modeled these daily observations by requiring at least 30 minutes between TOAs. This has the advantage of mitigating correlated $\sigma_{\text {DISS }}$ between the TOAs. We then compared the rms of these non-contiguous TOAs to the rms of an equivalent length of contiguous TOAs. The total contiguous length changes based on the overall length of the observation. As an example, for a 2.5 hour observation, if we require 30 minutes between each TOA, we will have five, non-contiguous, four minute integrated TOAs with 64 frequency channels, which will be compared to a contiguous observation of 20 minutes split into four-minute integrated TOAs and 64 frequency channels.

To compare equivalent length observations, we found the rms of the residuals 
of the first contiguous set of TOAs. We then shifted the start of the contiguous observation in time by one TOA (here four minutes), and again calculated the rms of the residuals, and so on until we reached the end of each full long observation.

For each set of noncontiguous TOAs we bootstrap sampled and calculated the rms of that bootstrapped set of residuals 10000 times. This bootstrapping allowed us to account for variations in TOA accuracy due to scintillation. We then shifted in time by one TOA (four minutes) and take each TOA separated by 30 minutes, bootstrap sampled and calculated the rms of the new set of residuals 10000 times, and so on until we exhausted all sets of TOAs that could be separated by 30 minutes.

\subsection{Scintillation Parameters and Secondary Spectra}

Here we present and discuss the results of both our scintillation parameter analysis and our secondary spectra analysis. We were able to measure both scintillation parameters for most MSPs with both the 1-D and 2-D fitting methods, however, for PSRs J0645+5158 and J1909-3744 we report only lower limits on $\Delta t_{\mathrm{d}}$. For all MSPs the 2-D method obtains more robust fits since there are many more points to fit despite the larger number of free parameters. The values we obtained for $\Delta \nu_{\mathrm{d}}$ compare well with the literature and predictions from NE2001, as do values of $\Delta t_{\mathrm{d}}$ when using transverse velocity measurements from pulsar timing. Additionally we find evidence that for both PSRs J0023-0923 and J1614-2230 the ISM differs from a purely uniform medium along the line of sight, and that for PSR J1614-2230 a single scattering screen is insufficient to describe the ISM along the pulsars line of 
sight. We were unable to find any scintillation arcs in the secondary spectra by-eye.

\subsubsection{Scintillation Parameters Results and Discussion}

Using both methods described in §3.3.2.1 we have determined scintillation timescales and bandwidths for each of the MSPs observed. The different parameters for each method for each MSP can be found in Table 3.2.

In general the 1-D parameter fitting agrees with the 2-D parameter fitting within $3 \sigma$. However, when summing over one axis, excess noise may be added to the 1-D ACF, making it difficult to fit a single Gaussian, whereas in the 2-D fit, there are many more samples being fit over, minimizing the excess noise. The 2-D Gaussian fit scintillation parameters also match previously obtained results in the literature within $3 \sigma$ in all cases. We can also analyze the scintle drift rates only by using the 2-D fits. Due to these factors, we have used the 2-D fit scintillation parameters for the remainder of this work.

For all of the binary MSPs analyzed in this work, the observation lengths are generally much shorter than the binary orbital periods, with the exception of PSR J0023+0923. We did not see any orbital-phase dependent changes in the scintillation properties as seen in some binary pulsars (Rickett et al., 2014; Reardon et al., 2019). 


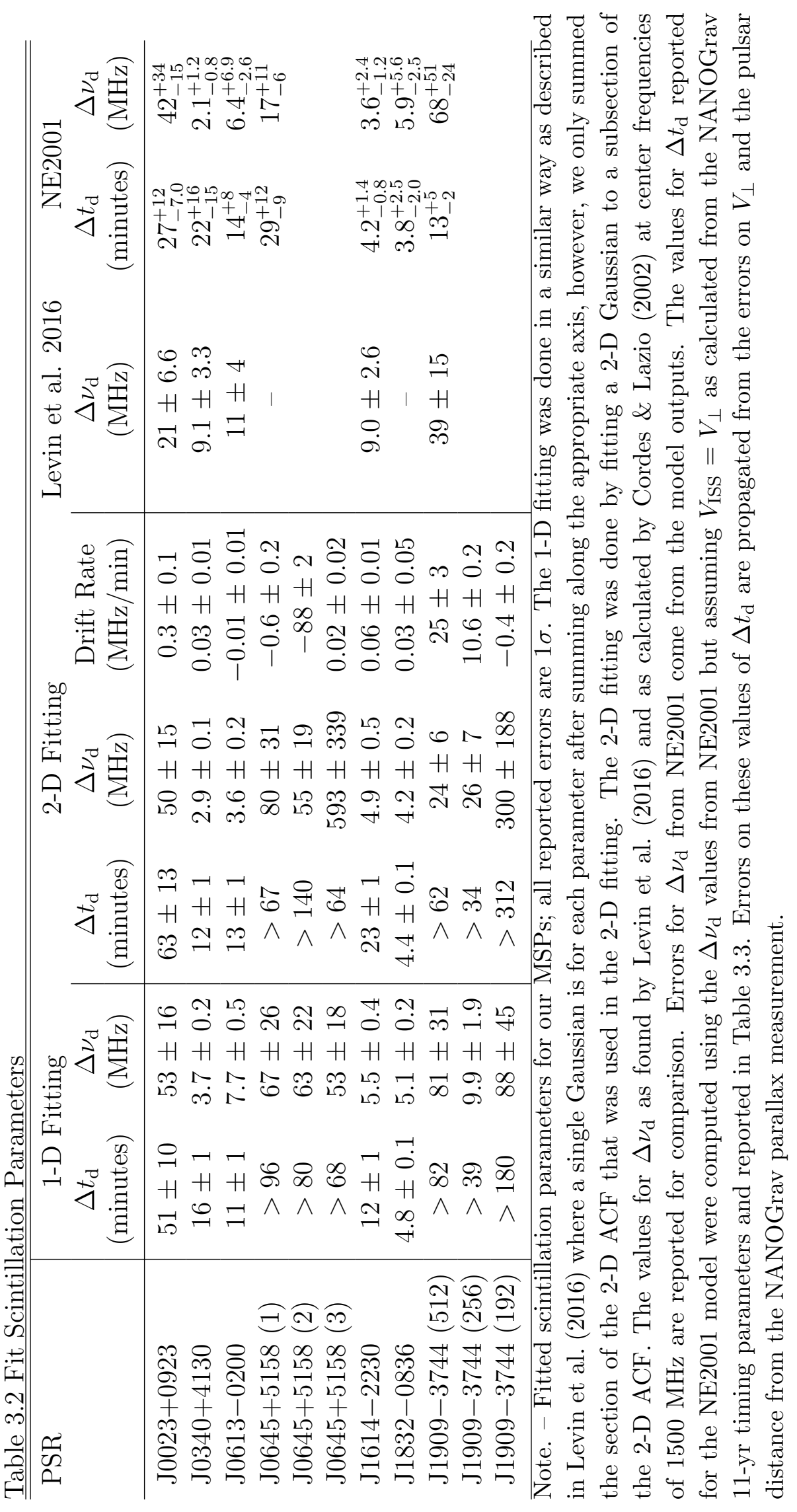


Table 3.3 Scintillation-derived Parameters

\begin{tabular}{|c|c|c|c|c|c|c|}
\hline PSR & $\begin{array}{c}D \\
(\mathrm{kpc})\end{array}$ & $\begin{array}{c}V_{\text {ISS }} \\
\left(\mathrm{km} \mathrm{s}^{-1}\right)\end{array}$ & $\begin{array}{c}V_{\perp} \\
\left(\mathrm{km} \mathrm{s}^{-1}\right)\end{array}$ & $\begin{array}{c}\tau_{\mathrm{d}} \\
(\mathrm{ns})\end{array}$ & $n_{\mathrm{ISS}}$ & $\begin{array}{c}\sigma_{\text {DISS }} \\
(\mathrm{ns})\end{array}$ \\
\hline J0023+0923 & $1.08 \pm 0.18$ & $33 \pm 6$ & $71 \pm 12$ & $3.2 \pm 1.0$ & $6 \pm 1$ & $1.3 \pm 0.4$ \\
\hline $\mathrm{J} 0340+4130$ & $1.4 \pm 0.9$ & $46 \pm 14$ & $22 \pm 14$ & $54.8 \pm 2.8$ & $240 \pm 10$ & $3.6 \pm 0.2$ \\
\hline J0613-0200 & $1.08 \pm 0.23$ & $44 \pm 5$ & $54 \pm 11$ & $43.6 \pm 2.3$ & $250 \pm 10$ & $2.8 \pm 0.2$ \\
\hline J0645+5158 (1) & $1.22 \pm 0.28$ & $41 \pm 9$ & $44 \pm 10$ & $2.0 \pm 0.8$ & $4 \pm 1$ & $1.1 \pm 0.4$ \\
\hline J0645+5158 (2) & & $16 \pm 4$ & & $2.9 \pm 1.0$ & $4 \pm 1$ & $1.4 \pm 0.5$ \\
\hline J0645+5158 (3) & & $120 \pm 40$ & & $0.3 \pm 0.2$ & $2 \pm 1$ & $0.2 \pm 0.1$ \\
\hline J1614-2230 & $0.67 \pm 0.04$ & $22 \pm 1$ & $103 \pm 7$ & $32.7 \pm 3.2$ & $79 \pm 8$ & $3.7 \pm 0.4$ \\
\hline J1832-0836 & $2.8 \pm 1.2$ & $220 \pm 50$ & $306 \pm 130$ & $37.9 \pm 1.4$ & $43 \pm 20$ & $1.8 \pm 0.1$ \\
\hline J1909-3744 (512) & $1.09 \pm 0.04$ & $24 \pm 3$ & $191 \pm 6$ & $6.5 \pm 1.5$ & $10 \pm 2$ & $2.1 \pm 0.5$ \\
\hline J1909-3744 (256) & & $44 \pm 6$ & & $6.1 \pm 1.7$ & $7 \pm 2$ & $2.4 \pm 0.7$ \\
\hline \multirow[t]{2}{*}{ J1909-3744 (192) } & & $16 \pm 5$ & & $0.5 \pm 0.3$ & $1 \pm 1$ & $0.5 \pm 0.3$ \\
\hline & $\begin{array}{l}\Omega_{\mathrm{u}, \Delta \nu_{\mathrm{d}}} \\
\left(\mathrm{mas}^{2}\right)\end{array}$ & $\begin{array}{l}\Omega_{\mathrm{u}, \Delta t_{\mathrm{d}}} \\
\left(\mathrm{mas}^{2}\right)\end{array}$ & $d_{s} / D$ & $\begin{array}{c}\Omega_{\mathrm{scr}} \\
\left(\mathrm{mas}^{2}\right)\end{array}$ & $\begin{array}{c}\theta_{r} \\
(\mathrm{mas})\end{array}$ & $\begin{array}{c}t_{\text {geo,min }} \\
(\mu \mathrm{s})\end{array}$ \\
\hline J0023+0923 & $0.017 \pm 0.006$ & $0.004 \pm 0.002$ & $0.30 \pm 0.13$ & $0.014 \pm 0.007$ & $0.22 \pm 0.15$ & $0.027 \pm 0.024$ \\
\hline $\mathrm{J} 0340+4130$ & $0.23 \pm 0.14$ & $0.9 \pm 1.1$ & $0.89 \pm 0.13$ & $0.4 \pm 0.4$ & $0.18 \pm 0.19$ & $0.45 \pm 0.33$ \\
\hline J0613-0200 & $0.24 \pm 0.05$ & $0.16 \pm 0.07$ & $0.57 \pm 0.12$ & $0.16 \pm 0.03$ & $-2.6 \pm 2.9$ & $12 \pm 24$ \\
\hline J1614-2230 & $0.28 \pm 0.03$ & $0.013 \pm 0.002$ & $0.083 \pm 0.015$ & $0.61 \pm 0.16$ & $9.4 \pm 2.9$ & $6.4 \pm 2.8$ \\
\hline J1832-0836 & $0.08 \pm 0.03$ & $0.04 \pm 0.03$ & $0.51 \pm 0.24$ & $0.05 \pm 0.02$ & $2.1 \pm 4.2$ & $20 \pm 60$ \\
\hline
\end{tabular}

Note. - Values derived from the scintillation parameters resulting from the 2-D fits in Table 3.2. We have calculated $V_{\text {ISS }}$, and compare that to $V_{\perp}$ from the NANOGrav 11-yr timing parameters. The distances to the pulsar $D$ from the NANOGrav parallax are also provided. Other parameters, $\tau_{\mathrm{d}}, n_{\mathrm{ISS}}$, and $\sigma_{\text {DISS }}$ are directly related to determining the white-noise contributions from the ISS. In the bottom section, we describe derived parameters from assuming the geometry of the medium along the line of sight to each pulsar is a uniform medium (scattering "strength" $\Omega_{\mathrm{u}}$ derived from $\Delta \nu_{\mathrm{d}}$ and $\Delta t_{\mathrm{d}}$, respectively), or a screen at a fractional distance from the Earth $d_{s} / D$ with strength $\Omega_{\text {scr }}$. The scintle drift rates from the 2-D fits provide the refraction angle along the pulsar's direction of motion $\theta_{r}$ and the corresponding geometric time delay $t_{\text {geo,min }}$ given the screen distance. 


\subsubsection{Scintillation Bandwidths}

When compared to Levin et al. (2016), the values obtained for $\Delta \nu_{\mathrm{d}}$ in this work match within $2 \sigma$. The discrepancies in these values are likely due to the fact that while the values of $\Delta \nu_{\mathrm{d}}$ obtained in Levin et al. (2016) were averaged over many epochs, the values reported in this work relate to a single epoch. As the ISM is a dynamic environment it is expected that the measured values of $\Delta \nu_{\mathrm{d}}$ will change in time, so some differences are expected. Additionally, our measurements of $\Delta \nu_{\mathrm{d}}$ better match those from Levin et al. (2016) for MSPs with fewer scintles. This may be because with a longer observation more scintles will be observed than in a shorter observation, but for MSPs with large $\Delta t_{\mathrm{d}}$ and $\Delta \nu_{\mathrm{d}}$, the numbers may be comparable. However, as we have a larger $n_{\mathrm{ISS}}$ in almost all cases we expect our parameters to be more accurate than those found in Levin et al. (2016).

For PSR J1909-3744, the value of $\Delta \nu_{\mathrm{d}}$ from the observation with only 192 frequency channels (300 MHz bandwidth) deviates greatly from the other values, spanning the full bandwidth of the observation. This discrepancy is likely due to both the smaller bandwidth and the lack of scintles observed in frequency for this section of the observation.

We report values of $\Delta \nu_{\mathrm{d}}$ for PSRs J0645+5158 and J1832-0836 for the first time in this work. For the two sections of the first observation of PSR J0645+5158, (1) and (2), the values of $\Delta \nu_{\mathrm{d}}$ agree with each other within $1 \sigma$. The third observation, (3), taken 10 days later, has a $\Delta \nu_{\mathrm{d}}$ almost spanning the full bandwidth, and different from the other two measurements by a factor of $\sim 12$. However, from 
the dynamic spectrum shown in Figure 3.1, we can see that for this observation we appear to only resolve a single scintle spanning the full bandwidth and length of the observation. The differences in $\Delta \nu_{\mathrm{d}}$ are therefore not surprising, and the actual value is likely closer to that of the first two segments. For J1832-0836, $\Delta \nu_{\mathrm{d}}$ is quite small, only about three times our frequency resolution.

When compared to the predicted values of $\Delta \nu_{\mathrm{d}}$ from NE2001, all of the values obtained from our long observations are within $2 \sigma$. While there is some variation, this shows the accuracy of the NE2001 model in predicting $\Delta \nu_{\mathrm{d}}$ along different lines of sight.

\subsubsection{Scintillation Timescales}

We also report values or lower limits of $\Delta t_{\mathrm{d}}$ for all seven MSPs for the first time. We note that our observation of PSR J0023+0923 contains only a small number of scintles (see Table 3.3). Additionally, due to the observing issues and length of the observations for both PSRs J0645+5158 and J1909-3744, there were no fully time resolved scintles, and the values reported here are at best lower limits on $\Delta t_{\mathrm{d}}$. For these two MSPs the $\Delta t_{\mathrm{d}}$ lower limit reported from 1-D fitting is the length of the observation.

For the NE2001 $\Delta t_{\mathrm{d}}$ estimates, we have calculated $\Delta t_{\mathrm{d}}$ using the same method as Cordes \& Lazio (2002), but have have used the proper motion velocity $V_{\perp}$ as determined from the NANOGrav 11-yr timing parameters as a proxy for $V_{\text {ISS }}$. These values are reported in Table 3.2 and match the derived values of $\Delta t_{\mathrm{d}}$ within $2 \sigma$ for 
all MSPs except PSRs J1614-2230 and J1909-3744. While it has been shown that $V_{\text {ISS }}$ and $V_{\perp}$ closely follow each other, differences in the two velocities can occur if the scattering does not occur uniformly along the line of sight (Lyne \& Smith, 1982; Cordes, 1986). Further analysis of these differences is beyond the scope of this work and left for future analyses.

\subsubsection{Uniform Media vs Thin Screens}

Following Appendix C of Cordes \& Rickett (1998), we can use the scintillation bandwidth and timescale to constrain the properties of the medium along the line of sight. We tested two geometries: a uniform medium and a thin scattering screen. For the latter, we can determine the distance and "strength" of the screen uniquely. The two parameters are related to these via

$$
\begin{aligned}
\tau_{\mathrm{d}} & =\frac{\eta_{0} \Delta s}{2 c} d_{s}\left(1-\frac{d_{s}}{D}\right), \\
\Delta t_{\mathrm{d}} & =\frac{\lambda}{\pi V_{\perp}}\left(\frac{1}{2 \eta_{0} \Delta s}\right)^{1 / 2} \frac{D}{d_{s}},
\end{aligned}
$$

where $\lambda=c / \nu$ is the electromagnetic wavelength and again $d_{s}$ is the Earth-screen distance. Recall that we can relate $\Delta \nu_{\mathrm{d}}$ to $\tau_{\mathrm{d}}$ via Eq. 3.6. We define the strength of the screen $\Omega_{\text {scr }} \equiv \eta_{0} \Delta s$ which is the product of the mean-square scattering angle per unit length along the line of sight times the thickness of the screen. The electron-density wavenumber spectrum has an amplitude proportional to $\eta_{0}$ (Cordes \& Rickett, 1998), and therefore when multiplied by the screen thickness $\Delta s$ gives the integrated scattering strength. The units of $\eta_{0}$ are often written in $\mathrm{mas}^{2} \mathrm{kpc}^{-1}$ 
and $\Omega$ has units of ass $^{2}$.

The scintillation parameters in the uniform medium case take a simpler form,

$$
\begin{aligned}
\tau_{\mathrm{d}} & =\frac{\eta_{0} D^{2}}{2 c} \\
\Delta t_{\mathrm{d}} & =\frac{\lambda}{\pi V_{\perp}}\left(\frac{3}{2 \eta_{0} D}\right)^{1 / 2} .
\end{aligned}
$$

For comparison with the thin-screen case, we can define $\Omega_{\mathrm{u}} \equiv \eta_{0} D$ to give the comparable scattering strength over the entire line of sight. If the $\Omega_{\mathrm{u}}$ obtained from both scintillation parameter measurements is consistent, then scattering is consistent with coming from throughout the line of sight.

Using the five MSPs in which we constrained both $\Delta t_{\mathrm{d}}$ and $\Delta \nu_{\mathrm{d}}$, we calculated the separate $\Omega$ values obtained for a uniform medium as well as the $d_{s} / D$ and $\Omega_{\text {scr }}$ values for the thin-screen geometry. These solutions are provided in Table 3.3. We see that for PSRs J0023+0923 and J1614-2230, the $\Omega$ values derived from the scintillation bandwidths and timescales are not consistent with each other, suggesting the line of sight differs from a purely uniform medium. These two MSPs have the tightest constraints on the $\Omega$ values, so it is possible that the lines of sight for all five of these MSPs differ slightly from a uniform medium, but we are unable to constrain them well enough to verify this.

\subsubsection{Scintle Drift Rates}

As discussed, we obtained scintle drift rates for the five MSPs in which we

performed the 2-D ACF fitting. We note that for PSR J1614-2230, we had to 
constrain the time-frequency space for the 2-D ACF fit. As shown in Figure 3.1, it appears that the drift rates are negative, or that the scintles are moving from higher to lower frequencies in time, in the first half of the observation at higher frequencies (with timescale $\gtrsim 1$ hour). This resulted in a smaller scale peak in the ACF with a positive drift rate, where the scintles appear to move from lower to higher frequencies with time, on top of a much larger feature with a negative drift rate. Upon closer inspection, we found that what appears to be two scintles in the top left of the dynamic spectrum (the beginning of the observation at higher frequencies) are actually several bright scintles with similar drift rates to the other scintles throughout the observation. This shows that a large number of scintles are required to measure scintillation parameters without a systematic bias; if we had a smaller bandwidth and/or a shorter observation time, we would have measured the scintillation parameters incorrectly. Since we believe that the shorter timescale is more representative of the characteristic scintillation timescale $\left(\Delta t_{\mathrm{d}} \sim 23 \mathrm{~min}\right)$, we constrained the 2-D Gaussian to fit only over the central portion of the ACF. For the other pulsars, we visually inspected the fits to ensure we were unbiased in our measurements and did not note another instance of this apparent drifting.

From the drift rates in the scintles, we can derive the refraction angle $\theta_{R}$ along the pulsar's direction of motion using Eq. 3.9. Since the refracted emission takes longer to travel to the pulsar, it is associated with a geometric time delay equal to (Cordes \& Shannon, 2010; Lam et al., 2016b)

$$
t_{\mathrm{geo}, \min }=\frac{1}{2 c}\left(\frac{D d_{s}}{D-d_{s}}\right) \theta_{r}^{2} .
$$


This is the minimum delay since we do not know the refraction angle in the direction perpendicular to the pulsar motion. Both $\theta_{r}$ and $t_{\text {geo,min }}$ are given in Table 3.3. Measurement of these geometric delays is critical; since the refraction delay is $\propto \nu^{-4}$, removal of the dispersive $\nu^{-2}$ delay will bias the "infinite-frequency" arrival times used in precision timing experiments (Lam et al., 2016b, 2018b). We found $t_{\text {geo,min }}$ was roughly consistent with zero for all pulsars after propagation of all uncertainties except for PSR J1614-2230. Assuming the delay is purely refractive and $\propto \nu^{-4}$, the value of $t_{\text {geo,min }}=6.4 \pm 2.8 \mu$ s implies a $\sim 70 \mu$ s delay at the $820 \mathrm{MHz}$ band also used by NANOGrav, and therefore a DM perturbation of amplitude $-0.015 \mathrm{pc} \mathrm{cm}^{-3}$ (Lam et al., 2018a), which is unseen in NANOGrav data (Arzoumanian et al., 2018c). We therefore believe that $t_{\text {geo,min }}$ is biased and neither a uniform medium nor single scattering screen are adequate to describe this line of sight; a more in-depth analysis on this pulsar's varying scintillation parameters using NANOGrav data will be performed in future work (M. T. Lam et al., in prep.).

\subsubsection{Estimating $\sigma_{\text {DISS }}$}

From the fit values of $\Delta t_{\mathrm{d}}$ and $\Delta \nu_{\mathrm{d}}$ we calculated values of $\tau_{\mathrm{d}}, \sigma_{\text {DISS }}$, and $n_{\mathrm{ISS}}$ from Eqs. 3.6, 3.7, and 3.8 respectively, which are reported in Table 3.3. For all MSPs where we are able to resolve $\Delta t_{\mathrm{d}}$, we report the most accurate values for $n_{\mathrm{ISS}}$ and $\sigma_{\text {DISS }}$ for this epoch. For MSPs where $\Delta t_{\mathrm{d}}$ is a lower limit, we took $\left(1+\eta_{t}\left(T / \Delta t_{\mathrm{d}}\right)\right) \approx 1$, as is typically done (Levin et al., 2016). We found that for all MSPs, $\sigma_{\text {DISS }}$ is on the order of nanoseconds, and smaller than or equal the values 
of $\sigma_{\text {DISS }}$ found by Lam et al. (2016a) and are thus a very small contribution to the white noise present in the timing residuals. The large number of scintles present in our long observations along with our new scintillation parameters likely account for the smaller $\sigma_{\text {DISS }}$ value.

\subsubsection{Secondary Spectra Results and Discussion}

No arcs were visually apparent in any of our observations, and therefore we did not further analyze the secondary spectra. While some pulsars, such as PSR J1614-2230, appear to be slightly brighter on one side of the zero conjugate frequency, there is no clear evidence of scintillation arcs. This could be due to a lack of frequency resolution in the dynamic spectra. However, with no obvious detection

of any arcs, further analysis of the secondary spectra is beyond the scope of this work.

\subsection{Pulse Jitter Results and Discussion}

Here we will present and discuss the results of our measurements of pulse jitter. We have attempted to measure the pulse jitter contribution to the white noise in our timing residuals using a direct method by fitting values of $\sigma_{\mathcal{R}}$ as a function of integration time as well as using a maximum likelihood analysis. While values of $\sigma_{\mathrm{J}}$ can be determined with both methods, in general, our TOAs do not have a high enough $\mathrm{S} / \mathrm{N}$ to separate out the $\sigma_{\mathrm{J}}$ contribution to the white noise. As a final test, we discuss the results of our sub-band correlation analysis, which is similarly 
Table $3.4 \sigma_{\mathcal{R}}$ and Integration Time Fitting

\begin{tabular}{l|c|cc|cc}
\hline \hline PSR & $\begin{array}{c}\text { Fixed } \sigma_{\mathrm{J}} \\
(\mathrm{ms})\end{array}$ & Fit $\alpha$ & $\begin{array}{c}\text { Fit } \sigma_{\mathrm{J}} \\
(\mathrm{ms})\end{array}$ & F statistic & F Significance \\
\hline $\mathrm{J} 0023+0923$ & $10.1 \pm 0.2$ & $-0.25 \pm 0.05$ & $13.4 \pm 0.2$ & 37.3 & 0.0009 \\
$\mathrm{~J} 0340+4130$ & $4.1 \pm 0.1$ & $-0.57 \pm 0.01$ & $4.6 \pm 0.1$ & 11.0 & 0.0162 \\
$\mathrm{~J} 0613-0200$ & $4.5 \pm 0.1$ & $-0.55 \pm 0.01$ & $4.8 \pm 0.1$ & 5.8 & 0.0526 \\
$\mathrm{~J} 0645+5158(1)$ & $1.7 \pm 0.1$ & $-0.62 \pm 0.01$ & $2.2 \pm 0.1$ & 23.1 & 0.0030 \\
$\mathrm{~J} 0645+5158(2)$ & $1.5 \pm 0.1$ & $-0.60 \pm 0.01$ & $1.8 \pm 0.1$ & 14.5 & 0.0089 \\
$\mathrm{~J} 0645+5158(3)$ & $1.6 \pm 0.1$ & $-0.55 \pm 0.02$ & $1.7 \pm 0.1$ & 13.3 & 0.0108 \\
$\mathrm{~J} 1614-2230$ & $1.0 \pm 0.1$ & $-0.53 \pm 0.01$ & $1.0 \pm 0.1$ & 7.7 & 0.0321 \\
$\mathrm{~J} 1832-0836$ & $0.6 \pm 0.2$ & $-0.48 \pm 0.01$ & $0.6 \pm 0.2$ & 35.1 & 0.0010 \\
$\mathrm{~J} 1909-3744(512)$ & $0.1 \pm 0.2$ & $-0.53 \pm 0.01$ & $0.1 \pm 0.2$ & 12.7 & 0.0119 \\
$\mathrm{~J} 1909-3744(256)$ & $0.4 \pm 0.2$ & $-0.55 \pm 0.01$ & $0.5 \pm 0.2$ & 4.6 & 0.0763 \\
$\mathrm{~J} 1909-3744(192)$ & $0.2 \pm 0.1$ & $-0.52 \pm 0.01$ & $0.2 \pm 0.1$ & 11.9 & 0.0136 \\
\hline
\end{tabular}

Note. - Estimated single pulse jitter values based on fitting for $\sigma_{\mathcal{R}}$ as a function of the integration time. Fixed values assume that $\sigma_{\mathcal{R}} \propto N^{-1 / 2}$, the number of pulses in the integration. Fit values are fitting for this dependency as $\sigma_{\mathcal{R}} \propto N^{\alpha}$. The F statistic and significance compare how significant fitting for the $\alpha$ value is to the fit. We use a significance of $0.0027(3 \sigma)$ to determine if fitting for $\alpha$ is significant. We find that it is significant for only two MSPs, PSRs J0023+0923 and J1832-1836.

hindered, despite a large sub-band width and integration time.

\subsubsection{Pulse Jitter Meausured from $\sigma_{\mathcal{R}}$}

Our first attempt to measure the pulse jitter is from direct calculation using Eq. 3.1 with $\sigma_{\mathcal{R}}$ calculated as a function of integration time and using measured values of both $\sigma_{\mathrm{S} / \mathrm{N}}$ and $\sigma_{\mathrm{DISS}}$. Figure 3.3 shows the two different fits of $\sigma_{\mathcal{R}}$, with the $1 / N^{\alpha}$ represented by the solid red line, and the $1 / \sqrt{N}$ by the dashed red line. We performed an F-test to calculate the significance of fitting a varying slope compared to a constant $\sqrt{N}$ to these $\sigma_{\mathcal{R}}$ as a function of integration time with a significance threshold of $0.0027(3 \sigma)$; the F-statistic and its significance are reported in Table 3.4 . 

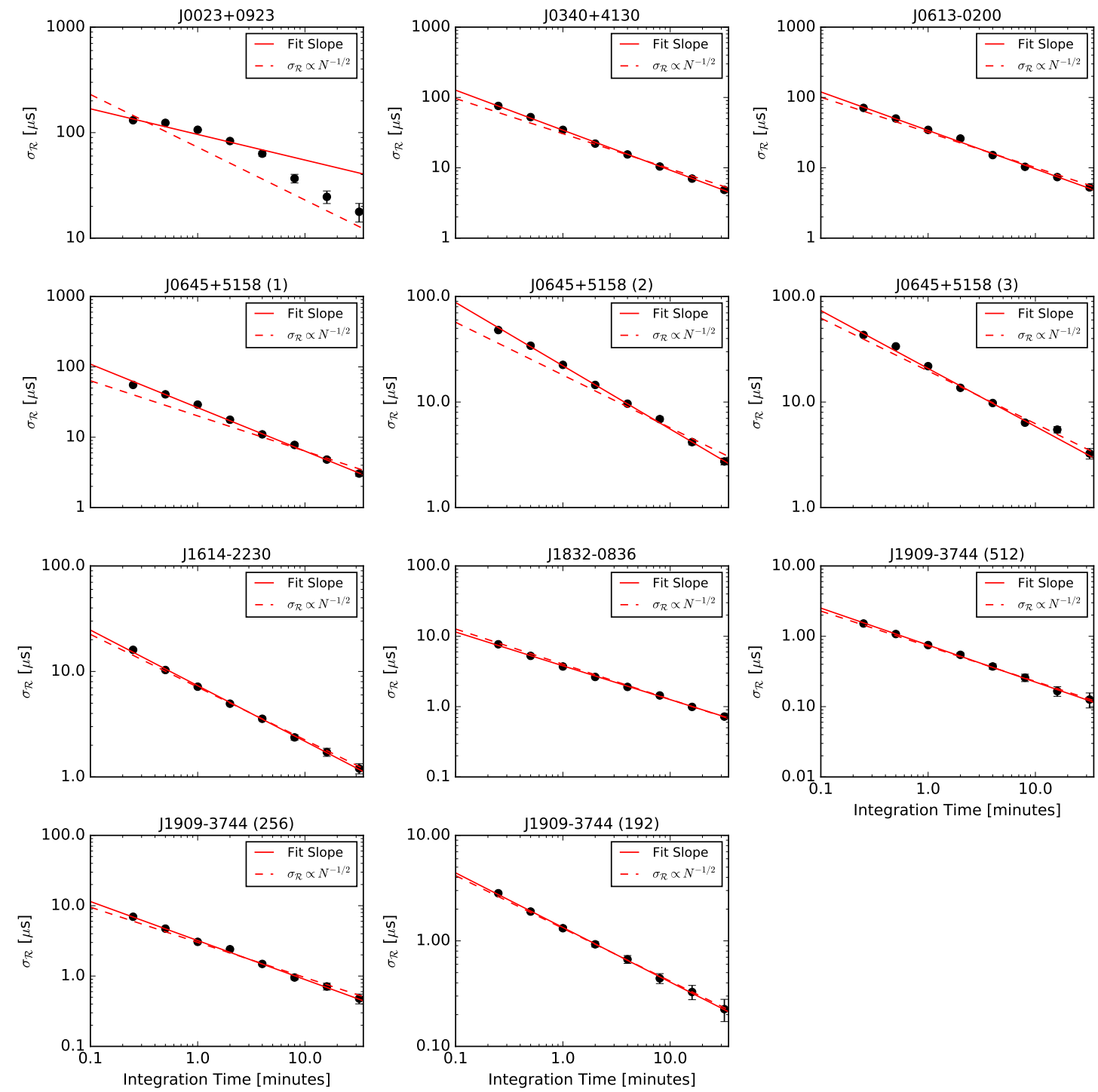

Figure $3.3 \sigma_{\mathcal{R}}$ as a function of integration time calculated from the residuals generated with PYPULSE for all MSPs analyzed in this work in log space. All points are potted with error bars, but not all are visible. The dashed red line shows $\sigma_{\mathcal{R}}$ $\propto N^{-1 / 2}$, where $N$ is the number of pulses in each folded integration and fit for just $\sigma_{\mathcal{R}}$ for single pulses. This is expected if we assume that all pulses from the pulsar are statistically independent. The solid line fits for both $\sigma_{\mathcal{R}}$ for single pulses and a dependence on $N^{\alpha}$, where $\alpha$ is the slope of the line in $\log$ space. The fit values for both of these lines are reported in Table 3.4. 
We find that fitting a slope instead of assuming a fixed $\sqrt{N}$ is significant for only two MSPs, PSRs J0023+0923 and J1832-0836. However, for PSR J1832-0836, $\alpha=-0.48 \pm 0.01$, which is within $2 \sigma$ of the expected value of -0.5 . For PSR J0023+0923, the shallower slope that is fit for shorter integration times is indicative that the individual pulses may not be statistically independent (Helfand et al., 1975; Rathnasree \& Rankin, 1995). Despite this apparently significant fit, we found that $\sigma_{\mathcal{R}}$ for a single pulse is consistent within $1 \sigma$ whether we fit for the slope or not. We will therefore discuss just the values obtained when assuming that $\sigma_{\mathcal{R}} \propto 1 / \sqrt{N}$ for the remainder of this work.

We report the values of $\sigma_{\mathcal{R}}, \sigma_{\mathrm{S} / \mathrm{N}}$, and $\sigma_{\mathrm{J}}$ for both two minute integrations with 64 frequency channels $(12.5 \mathrm{MHz}$ per channel) as well as the values extrapolated back for single pulses. For $\sigma_{\mathrm{S} / \mathrm{N}}$ we took the value of $S$ in Eq. 3.4 to be the median value of $S$, calculated as the peak to off-pulse rms ratio, for all the TOAs used in each set of integrations. For some MSPs this results in $\sigma_{\mathrm{S} / \mathrm{N}}>\sigma_{\mathcal{R}}$. If no value is reported then we were unable to determine a value of $\sigma_{\mathrm{J}}$ with this method. However, this result in general shows that we cannot assume any TOAs from a given observation will be in the high $\mathrm{S} / \mathrm{N}$ regime, meaning $\sigma_{\mathrm{S} / \mathrm{N}} \gg \sigma_{\mathrm{J}}$.

\subsubsection{Maximum Likelihood Jitter Results}

Our second attempt at estimating the pulse jitter from the maximum likelihood analysis also found results similar to those presented above. While a statistically significant value for the pulse jitter was determined using this analysis, $\sigma_{\mathrm{S} / \mathrm{N}}$ was 
Table $3.5 \sigma_{\mathrm{J}}$ Estimates from Fitting

\begin{tabular}{|c|c|c|c|c|c|c|c|c|c|}
\hline \multirow[t]{2}{*}{ PSR } & \multirow{2}{*}{$\begin{array}{l}W_{\text {eff }} \\
(\mu \mathrm{s})\end{array}$} & \multicolumn{2}{|c|}{ Max Likelihood } & \multicolumn{3}{|c|}{2 Minute Integrations } & \multicolumn{3}{|c|}{ Single Pulse } \\
\hline & & $\begin{array}{c}\sigma_{C} \\
(\mu \mathrm{s})\end{array}$ & $\begin{array}{c}\sigma_{\mathrm{J}} \\
(\mu \mathrm{s})\end{array}$ & $\begin{array}{c}\sigma_{\mathcal{R}} \\
(\mu \mathrm{s})\end{array}$ & $\begin{array}{c}\sigma_{\mathrm{S} / \mathrm{N}} \\
(\mu \mathrm{s})\end{array}$ & $\begin{array}{c}\sigma_{\mathrm{J}} \\
(\mu \mathrm{s})\end{array}$ & $\begin{array}{c}\sigma_{\mathcal{R}} \\
(\mathrm{ms})\end{array}$ & $\begin{array}{c}\sigma_{\mathrm{S} / \mathrm{N}} \\
(\mathrm{ms})\end{array}$ & $\begin{array}{c}\sigma_{\mathrm{J}} \\
(\mathrm{ms})\end{array}$ \\
\hline $\mathrm{J} 0023+0923$ & 430 & $9.6_{-3.2}^{+3.0}$ & 9.6 & $83 \pm 2$ & $43 \pm 46$ & 71 & $10.1 \pm 0.2$ & 8.6 & 5.4 \\
\hline $\mathrm{J} 0340+4130$ & 517 & $4.8_{-0.9}^{+0.9}$ & 4.8 & $22.1 \pm 0.4$ & $23 \pm 13$ & - & $4.1 \pm 0.1$ & 4.4 & - \\
\hline J0613-0200 & 332 & $5.4_{-0.8}^{+0.8}$ & 5.4 & $26.1 \pm 0.5$ & $24 \pm 18$ & 11 & $4.5 \pm 0.1$ & 4.7 & - \\
\hline J0645+5158 (1) & 633 & $7.4_{-0.8}^{+0.8}$ & 7.4 & $17.7 \pm 0.4$ & $16 \pm 8$ & 8 & $1.7 \pm 0.1$ & 1.8 & - \\
\hline J0645+5158 (2) & 633 & $5.8_{-0.6}^{+0.6}$ & 5.8 & $14.5 \pm 0.3$ & $13 \pm 7$ & 6 & $1.5 \pm 0.1$ & 1.5 & - \\
\hline J0645+5158 (3) & 633 & $4.5_{-0.5}^{+0.5}$ & 4.5 & $13.6 \pm 0.3$ & $12 \pm 5$ & 6.6 & $1.6 \pm 0.1$ & 1.4 & 0.8 \\
\hline J1614-2230 & 403 & $4.9_{-0.2}^{+0.2}$ & 4.9 & $5.0 \pm 0.2$ & $6 \pm 5$ & - & $1.0 \pm 0.1$ & 1.2 & - \\
\hline J1832-0836 & 188 & $0.7_{-0.1}^{+0.1}$ & 0.7 & $2.6 \pm 0.1$ & $3 \pm 2$ & - & $0.6 \pm 0.2$ & 0.7 & 0.2 \\
\hline J1909-3744 (512) & 266 & $0.1_{-0.1}^{+0.1}$ & 0.1 & $0.6 \pm 0.1$ & $0.9 \pm 0.8$ & - & $0.1 \pm 0.2$ & 0.2 & 0.2 \\
\hline J1909-3744 (256) & 266 & $1.6_{-0.2}^{+0.2}$ & 1.6 & $2.4 \pm 0.1$ & $2.7 \pm 1.7$ & - & $0.4 \pm 0.2$ & 0.5 & 0.2 \\
\hline J1909-3744 (192) & 266 & $0.1_{-0.1}^{+0.1}$ & 0.1 & $0.9 \pm 0.1$ & $1.5 \pm 1.4$ & - & $0.2 \pm 0.1$ & 0.3 & 0.2 \\
\hline
\end{tabular}

Note. - Estimates of the pulse jitter from the fit of the rms of the timing residuals as shown in Figure 3.3 and compared with the results from the maximum likelihood analysis. The values of $W_{\text {eff }}$ and $\sigma_{\text {DISS }}$ are the same as reported in Table 3.3. The values used for $\sigma_{\mathrm{S} / \mathrm{N}}$ and $\sigma_{\mathcal{R}}$ are reported for both the two minute integration residuals and the values extrapolated for single pulses. If no value is reported for $\sigma_{\mathrm{J}}$, this method is unable to estimate $\sigma_{\mathrm{J}}$. Maximum likelihood values are taken for $50 \mathrm{MHz}$ wide frequency channels and two minute integrations.

found to be larger than $\sigma_{\mathrm{J}}$ in all cases reinforcing that our high $\mathrm{S} / \mathrm{N}$ regime assumption does not hold for these observations. Despite the limiting $\mathrm{S} / \mathrm{N}$ of our TOAs, the results of the maximum likelihood pulse jitter analysis are reported in Table 3.5.

The maximum likelihood analysis found that in all cases the values of $\sigma_{C}^{2}$ are significant and are not $95 \%$ upper limits. Using our measurements of $\sigma_{\text {DISS }}$ we could separate out the $\sigma_{\mathrm{J}}$ from $\sigma_{C}^{2}$. However, since $\sigma_{C}^{2} \gg \sigma_{\text {DISS }}$ as estimated from the dynamic spectra in all cases, the majority of the contribution to $\sigma_{C}^{2}$ appears to be from the pulse jitter. We note that all values of $\sigma_{\mathrm{J}}$ found here are much larger than those found by Lam et al. (2016a) and Lam et al. (2019) and are not representative of the best constraints that may be placed on the pulse jitter of these seven MSPs. Even so, using a maximum likelihood analysis better constrains $\sigma_{\mathrm{J}}$ than directly 
solving Eq. 3.1.

While our values of pulse jitter from the maximum likelihood analysis are larger than expected, Lam et al. (2016a) used the full NANOGrav 9-yr data set in their analysis, and Lam et al. (2019) used the full NANOGrav 12.5-yr data set, which allows for many more TOAs than our single long observations. The longer 9-yr/12.5yr data set also means there is a greater chance of observing the pulsar during a particularly bright DISS time, or during a period of strong refractive scintillation (RISS), which can increase the observed flux density of the pulsar by factors of $\sim 2$ (Stinebring et al., 2000).

The typical timescale of RISS is typically days to weeks, increasing with pulsar distance (Sieber, 1982; Rickett et al., 1984; Hancock et al., 2019). With just one epoch of observation, even spanning many hours, we are unlikely to have observed during a period of strong RISS for any pulsar when compared to many observations spanning multiple epochs. This, in addition to the low likelihood of observing during a period of bright DISS, are likely the primary explanations for the lower $\mathrm{S} / \mathrm{N}$ of our observations and our ability to put limits on the pulse jitter.

\subsubsection{Sub-band Correlation Results}

We expect that at high $\mathrm{S} / \mathrm{N}$ we will be able to see pulse jitter correlated across frequency channels as in Shannon \& Cordes (2012); Shannon et al. (2014). However, even with 16 min integrations and two frequency channels of $400 \mathrm{MHz}$ each, the correlations between the TOAs in the two sub-bands are minimal. The 
correlation coefficients are small which is indicative of not being able to detect the pulse jitter in our observations.

This lack of TOA correlations between sub-bands is not surprising given the low pulse $\mathrm{S} / \mathrm{Ns}$ discussed in $§ 3.5 .2$. Since we must be in the high $\mathrm{S} / \mathrm{N}$ limit to see indications of pulse jitter in the sub-band correlations, we do not meet our initial assumptions necessary for this analysis.

\subsection{DM Variations on Short Timescales}

We found that for almost all MSPs analyzed, the DM is consistent within $1 \sigma$ throughout the observation, and all points are consistent within $2 \sigma$, as expected from Jones et al. (2017). DM variations referenced to the nominal DM value from the NANOGra 11-yr timing parameters and reported in Table 3.1 are shown in each panel in Figure 3.4. For all MSPs our fit DM value differs from the value in the NANOGrav 11-yr data set at the same or closest epoch on the order of $\sim 10^{-3} \mathrm{pc} \mathrm{cm}^{-3}$ or less.

Here we have taken our $1 \sigma$ uncertainties directly from the TEMPo fitting. We note that the $\mathrm{S} / \mathrm{N}$ of the pulses will vary across the frequency band and with time due to scintillation. However, for PSRs J0340+4130, J0613-0200, J1614-2230, and J1832-0836, both $\Delta t_{\mathrm{d}}$ and $\Delta \nu_{\mathrm{d}}$ are smaller than the integration time of 32 minutes and frequency channel width of $12.5 \mathrm{MHz}$, so we expect the $\mathrm{S} / \mathrm{N}$ to be roughly the same for each TOA. For the other MSPs, we expect that any variations in S/N across the band or with time should be accounted for with larger or smaller uncertainties 

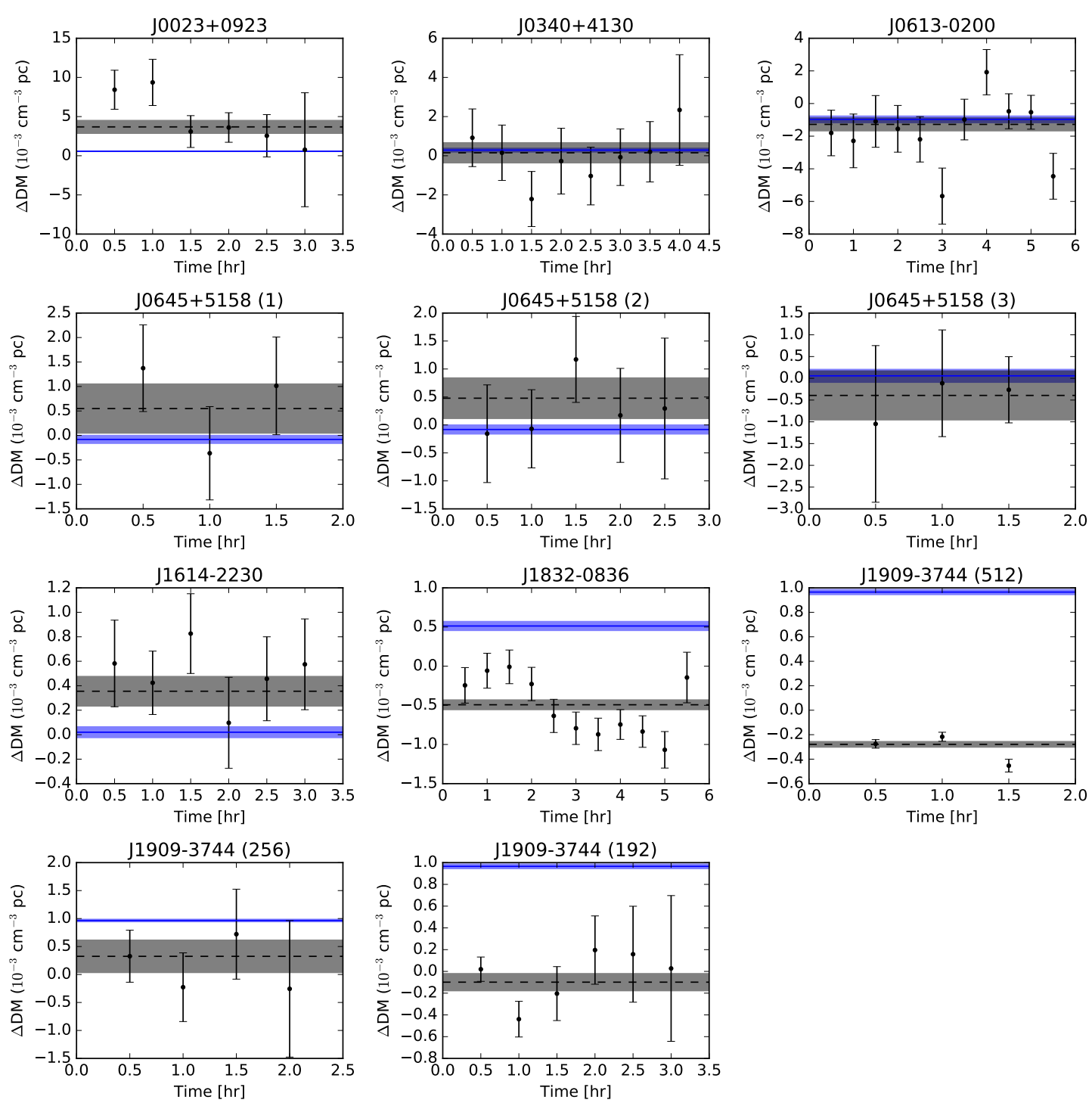

Figure 3.4 DM variations referenced to the nominal DM value from the NANOGrav 11-yr timing parameters and reported in Table 3.1 over the course of each long observation analyzed in this work. The blue line is the DM value from the NANOGrav 11-yr data set from the same or closest epoch to our observations and the blue filled region shows the $1 \sigma$ errors. The black dashed line is the DM value fit from TEMPO over the full long observation where the black filled region shows the $1 \sigma$ errors also from TEMPO. The black points are the fit DM value of using 32 minute integrations of the long observation with $1 \sigma$ errorbars from TEMPO. Three panels are shown for PSR J1909-3744 because the observation was split into three sections of different bandwidths due to data acquisition instrumental difficulties when recording the data. Differences between the fit values in this work and those from NANOGrav are likely due to the larger frequency band used to fit the DM in the NANOGrav data. In most cases there is little variation over the course of the observation and all fit $\Delta \mathrm{DM}$ values are consistent within $2 \sigma$. 
on the fit DM.

Using the estimation technique described in $§ 3.3 .6$, we have also determined what the expected variation in DM should be for each MSP. To do this we have used only the longest observation length for each MSP if they have multiple observations, and have used $V_{\text {ISS }}$ for all MSPs where we were able to measure $\Delta t_{\mathrm{d}}$, and $V_{\perp}$ otherwise. With distances between 0.67 and $2.4 \mathrm{kpc}$, observation lengths of 2.46 to 5.76 hours, and transverse velocities of 22 to $220 \mathrm{~km} \mathrm{~s}^{-1}$, we find that the DM variations expected from the ISM for the MSPs analyzed here range between $2-15 \times 10^{-8} \mathrm{pc} \mathrm{cm}^{-3}$ assuming a frequency of $1500 \mathrm{MHz}$. This is significantly smaller than any variations we can measure, and reinforces our expectation that the DM will not vary on hour-long timescales.

The difference between our fit DM and the NANOGrav 11-yr DM values is likely due to the fact that while we are fitting for a single DM value over the full bandwidth at one epoch, the NANOGrav DM values are fit using six day bins which often include additional observations taken at other frequency bands (Arzoumanian et al., 2015). Our smaller frequency range likely biases our DM fit which would account for the difference between the two DM values. This shows the importance of fitting the DM over as large a frequency range as possible.

While most fit DM value are within $1 \sigma$ of the expected DM value from our full observation DM fit, some fall $2 \sigma$ away. One possible explanation for the larger DM differences on these short timescales could be changes in the ionosphere. However, Lam et al. (2016b) has shown these variations to be on scales much smaller than our fit DM differences. It is also possible that variations in the pulse profile over 
the course of our observation could cause these variations. However, Brook et al. (2018) have shown that for the MSPs presented in this work the profile variations are very small and therefore unlikely sources of these variations.

Using Eq. 3.12 we can find what the peak pulse profile shift in time would need to be to account for the DM difference in the 32 minutes integrations and the full observation DM fit. For the largest DM difference we find, $0.0056 \mathrm{pc} \mathrm{cm}^{-3}$ for PSR J0023+0923, we find a shift of $10 \mu$ s from the expected TOA would be required to explain the difference in DM. For this MSP, our observation covers about $85 \%$ of its binary orbit. However, using the NANOGrav 11-yr data set we find no correlation between the DM variations and orbital phase for PSR J0023+0923, so we do not believe that the variations we find here are due to the orbital phase of the MSP.

For most other $2 \sigma$ DM differences we find, peak pulse profile shifts on the order of $1 \mu \mathrm{s}$ are required, which is of the order of the timing precision of most NANOGrav MSPs (Arzoumanian et al., 2018c) and therefore variations at this level are expected. Intrinsic variations in the pulse profile with frequency or variations due to scattering may also account for these shifts.

As no MSPs in this work show DM variations larger than $2 \sigma$ from the expected DM value over each observation, which can be accounted for as discussed above, we conclude that the DM of these MSPs does not vary on hour-long timescales. 

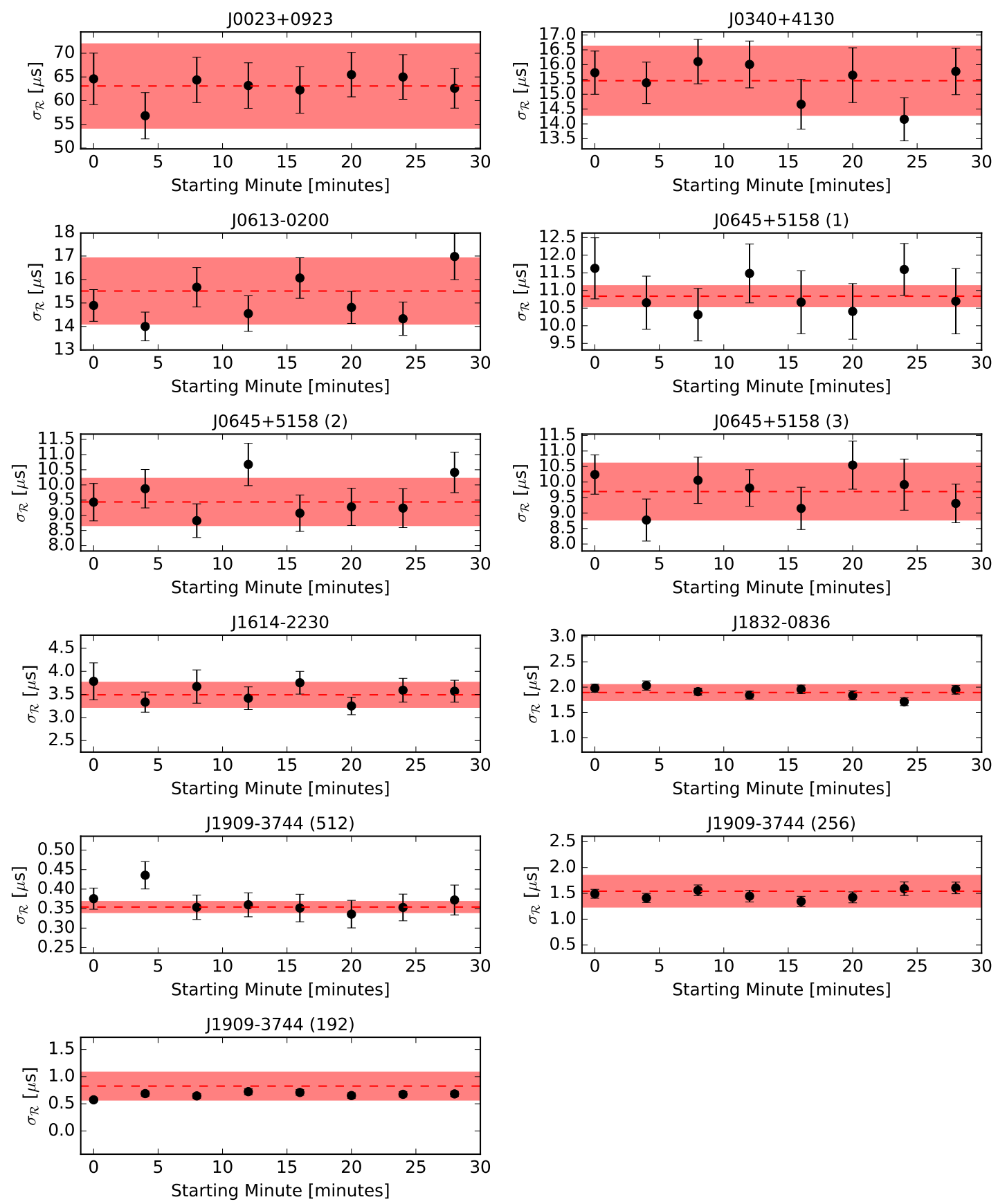

Figure 3.5 Comparison of $\sigma_{\mathcal{R}}$ for non-contiguous sets of pulsar timing residuals to contiguous set of timing residuals of equivalent length for each observation of each MSP. The red dashed line is the mean rms of the timing residuals for all equivalent contiguous sets of timing residuals and the red shaded region shows the one sigma standard deviation of the same. Each black point is the rms of a set of four minute integrated TOAs over 64 frequency channels separated by at least 30 minutes. The errorbars come from the standard deviation of $\sigma_{\mathcal{R}}$ calculated from $10^{5}$ bootstrapped samples of the 30 minute separated TOAs. In each case there appears to be little difference between taking residuals from a contiguous observation and from noncontiguous observations. 


\subsection{Timing Precision of Non-contiguous Timing Residuals Results and Discussion}

For all MSPs in our sample we found that there was little difference in the rms of the timing residuals, $\sigma_{\mathcal{R}}$, when using contiguous TOAs when compared to non-contiguous TOAs as expected. Errors on the rms of each set of residuals comes from the standard deviation of the distribution of rms residuals. All sets of noncontiguous timing residuals match within $1 \sigma$ of the expected $\sigma_{\mathcal{R}}$ for our choice of 64 frequency channels and four minutes integrations, with the exception of one set of timing residuals from the full bandwidth observation of PSR J1909-3744, as shown in Figure 3.5. The rms of the residuals here is larger than is seen in the NANOGrav data sets due to the smaller integration time used here (Arzoumanian et al., 2015, 2018c).

It is possible that this particular set of non-contiguous residuals suffers from blower than average $\mathrm{S} / \mathrm{N}$ compared to other sets of residuals, or from small amounts of RFI contamination, leading to larger errors on the timing residuals for this set, although there is nothing obvious for this set that shows this. As this point is still within $2 \sigma$ of the expected $\sigma_{\mathcal{R}}$, and all other points are with $1 \sigma$, this point shows only that it is possible to get unlucky scintillation or RFI during observations.

This result is promising as it suggests that time-continuity of TOAs has a small, if any, effect on precision pulsar timing. While the gaps between CHIME TOAs will be much larger than those in this study, it suggests that they may not significantly affect the achievable timing precision. Additionally, if we had found 
DM variations on hour-long timescales it could pose problems for CHIME to track daily DM changes. There are of course many other considerations such as pulse profile evolution and observing frequency that will have to be considered along with these effects.

Our results do show that if sections of the observation need to be dropped due to RFI contamination, the timing residual precision will not be affected beyond the expected $\sigma_{\mathcal{R}} \propto 1 / \sqrt{N}$. Additionally, if observations where a pulsar were only observed for short periods of time to try to observe only when it is scintillating brightly, the timing residual precision would not be affected due to the on-off nature of this observation, and would be improved due to higher $\mathrm{S} / \mathrm{N}$ of brightly scintillated pulses.

\subsection{Conclusions}

We have examined various noise parameters commonly seen in pulsar timing residuals, determined scintillation parameters, some for the first time, looked for DM variations on hour-long timescales, and analyzed the impact of non-contiguous timing residuals using a unique set of multi-hour continuous observations of seven different MSPs. The major conclusion from our analyses are summarized below.

- We present new measurements of $\Delta \nu_{\mathrm{d}}$ for all MSPs in our sample, some for the first time, as well as measurements and lower limits of $\Delta t_{\mathrm{d}}$ for all MSPs for the first time. We find that 2-D Gaussian fitting gives more robust scintillation parameters than 1-D Gaussian fitting. We also find that the scintle drift rates 
for PSRs J0023+0923 and J1614-2230 suggests that the line of sight is not well modeled by a uniform medium, and for J1614-2230 a single scattering screen is not sufficient to describe the line of sight. Additionally we are able to report values of $\sigma_{\text {DISS }}$ in a regime where we are not dominated by the finite scintle effect for some of the MSPs in our sample.

- We estimate $\sigma_{\mathrm{J}}$ of all MSPs using two different methods and find that the maximum likelihood method yields a more constraining result. Additionally we are limited by the low $\mathrm{S} / \mathrm{N}$ of our observations, showing the importance of refractive scintillation on estimating pulse jitter at higher frequencies.

- We find the DM measured for each MSP in our sample does not vary within our sensitivity limits on timescales of hours, as expected.

- There is little difference in $\sigma_{\mathcal{R}}$ of timing residuals that are non-contiguous in time when compared with equivalent timing residuals that are contiguous. Our result is promising for instruments like CHIME restricted to short but frequent observations and also show that should a section of an observation be removed, the timing precision of the residuals will not be significantly affected.

- Given the results, particularly of the pulse jitter and DM variations on these long observations, we find that the TOA variations in these long data sets are consistent with the assumed breakdown into template-fitting error, jitter error, and DISS error, despite the limiting $\mathrm{S} / \mathrm{N}$ of the data set.

The nature of a continuous long observation of a single pulsar, particular an 
MSP, allows us to study a wide variety of noise parameters commonly seen in pulsar timing as well properties of the ISM that are often difficult to otherwise probe. Additionally, continuous long observations are the only way to measure the scintillation timescale of many pulsars, an important characteristic for determining white noise caused by scintillation in pulsar timing residuals, and necessary to quantify for precision pulsar timing. In order to better constrain the noise parameters of MSPs as well as probe the ISM, it will be necessary to perform continuous long observations of many MSPs at multiple wavelengths. 


\section{Chapter 4}

\section{A Study in Frequency Dependent Effects on Precision Pulsar Timing Parameters with the Pulsar Signal Simulator}

\subsection{Introduction}

Precision timing of millisecond pulsars (MSPs) has allowed us to study some of the most extreme astrophysical phenomena, from the equations of state of neutron stars (e.g., Antoniadis et al., 2013; Stovall et al., 2018; Cromartie et al., 2020) to some of the most rigorous tests of general relativity (e.g., Kramer et al., 2006; Archibald et al., 2018; Zhu et al., 2019). MSPs have also been used to study the properties and dynamics of the interstellar medium (ISM; e.g., Levin et al., 2016; Jones et al., 2017; Lam et al., 2019; Shapiro-Albert et al., 2020). Pulsar timing arrays (PTAs) made up of MSPs are used by the North American Nanohertz Observatory for Gravitational Waves (NANOGrav; McLaughlin, 2013), the European Pulsar Timing Array (EPTA; Kramer \& Champion, 2013), and the Parkes Pulsar Timing Array (PPTA; Hobbs, 2013) to search for gravitational waves (GWs) from supermassive black hole binary systems (e.g., Shannon et al., 2013; Zhu et al., 2014; Lentati et al.,

2015; Shannon et al., 2015; Arzoumanian et al., 2016; Babak et al., 2016; Verbiest

Published as B. J . Shapiro-Albert et al. 2021, ApJ, 909, 2.

Contributing authors: J. S. Hazboun, M. A. McLaughlin, M. T. Lam 
et al., 2016; Arzoumanian et al., 2018a; Aggarwal et al., 2019; Arzoumanian et al., 2020a,b).

For experiments focused on GW detection and characterization, the characterization of noise in the detector is critical (Cordes \& Shannon, 2010; Cordes, 2013; Lam, 2018; Hazboun et al., 2019). There are many sources which may contribute to the uncertainty of a pulse time of arrival (TOA), making these detections challenging (e.g., Lam, 2018). In particular, various frequency-dependent effects due to both the ISM and the emission at the MSP may increase the uncertainty of a pulse TOA. These include variations in the dispersion measure (DM), i.e., the integrated column density of free electrons along the line of sight. Time delays due to dispersion are $\propto \mathrm{DM} \times \nu^{-2}$, where $\nu$ is the frequency of the radio emission; these variations may result in excess noise if they are not modeled appropriately (e.g., Jones et al., 2017; Lam et al., 2018b). Similarly, pulse scatter broadening due to inhomogeneities in the ISM will also cause time-variable delays. Scattering delays are expected to be $\propto \nu^{-4}$ (e.g., Shannon \& Cordes, 2012; Lam et al., 2019) and will also result in excess noise if not modeled or mitigated. Finally, evolution of the pulse shape with frequency may also increase the uncertainty of the pulse TOAs if it is not well modeled (e.g., Kramer et al., 1998; Pennucci et al., 2014).

In pulsar timing the time variations in pulsar DM are often modeled by fitting for a $\Delta \mathrm{DM}$, as an epoch-dependent offset from a fiducial DM value. The model for DM variations used in NANOGrav data sets is a piecewise-constant set of offsets, referred to as 'DMX', with a value for each observing epoch (e.g., Arzoumanian et al., 2016; Jones et al., 2017; Arzoumanian et al., 2018c). However, accounting for effects 
such as scattering and profile evolution are more difficult. To account for profile evolution, Frequency-Dependent, or "FD", parameters, polynomial coefficients in log-frequency space, along with a JUMP parameter which accounts for additional unmodeled profile evolution and other effects between low and high frequency data, are typically added to the pulsar timing model (Zhu et al., 2015; Arzoumanian et al., 2016).

The number of FD parameters fit varies for each MSP (Arzoumanian et al., 2016) but all terms are expected to be covariant with any other frequency-dependent timing parameters, including DMX and the JUMP parameter. While it is generally assumed that the largest component of the frequency-dependent time delay accounted for by FD parameters is due to intrinsic pulse profile evolution with frequency (Zhu et al., 2015), FD parameters will also account for the average scattering broadening over the course of a data set.

Here we present an analysis of the covariance between the DMX and FD parameters, as well as of the contributions of non- $\nu^{-2}$ effects to both the FD and DMX parameters using simulated data generated with the PUlsar Signal Simulator ${ }^{1}$ (PSRSIGSIM) PYTHON package (Hazboun et al., 2020). The PSRSIGSIM allows us to directly simulate variations in DM, frequency-dependent pulse profile evolution, and pulse scatter broadening to directly quantify how each of these contributions affects the recovered timing model parameters. Using simulated data allow us to constrain the impacts of any simulated effects on timing model parameters, precision pulsar timing, and the covariances between the frequency-dependent effects.

\footnotetext{
${ }^{1}$ https://github.com/PsrSigSim/PsrSigSim
} 
We briefly describe the PSRSIGSIM package in $§ 4.2$. In $\S 4.3$ we describe our data analysis pipeline. Our various simulated data sets are described in $\S 4.4$ and the results and analysis of the simulated data are presented in $\S 4.5$. The implications of our results on precision pulsar timing are presented in $\S 4.6$. Finally, we present concluding remarks and future work in $\S 4.7$.

\subsection{PsrSigSim Description}

The PSRSIGSIM is a PYTHON-based package designed to simulate a realistic pulsar signal including emission at the pulsar, transmission through the ISM, observation by a radio telescope, and output of a data file (Hazboun et al., 2020). Simulations are run on an observation by observation basis and can be run multiple times to create multiple epochs of data. The PSRSIGSIM has a variety of uses for educational purposes (Gersbach \& Hazboun, 2019), but here we focus on its use as a scientific simulation tool in this chapter.

The package includes modules for various signal classes which define attributes of the signal and observation such as the center frequency, bandwidth, number of frequency channels, and, for the FILTERBANKSIGNAL class which is used in this work, the number of subintegrations and their length. All signAL classes also have an option for the number of polarizations, however the PSRSIGSIM current only supports total intensity signals, assumed to be the sum of two polarizations. The PSRSIGSIM also enables single-pulse simulations using the FILTERBANKSIGNAL class, though not used for this work. 
The PULSAR class is used to define the properties intrinsic to the pulsar, such as the period $(P)$, the mean flux $\left(S_{\text {mean }}\right)$ and its reference frequency, and the spectral index $(\alpha)$. In order to define a pulse profile, the PULSAR class makes use of either a PROFILE class, for a single profile to be used at all frequency channels, or a PORTRAIT class, for a 2-D, frequency-dependent pulse-profile array. The profiles can be defined in these classes either through the amplitude, position, and width of any number of Gaussians, by defining a function that describes the profile shape as a function of phase, or by supplying a data array representative of the pulse shape. To define the pulse profile, the PULSAR class takes one of these PROFILE or PORTRAIT classes.

The ISM class is used for modeling the effects of the ISM on the pulsar signal and also account for intrinsic profile evolution. It includes attributes such as DM, FD parameters, and scattering timescale. The ISM class enables various signal processing techniques, e.g., the shift theorem, to add radio-frequency dependent delays. The PSRSIGSIM adds these delays to the pulses at specific points of the simulation dependent on astrophysical and efficiency considerations. Use of Fourier-based techniques allows the PSRSIGSIM to account for time delays that have time shifts which are fractional in phase bins. In the case of scatter broadening, the input scattering timescale is scaled as a function of frequency based on both a user input reference frequency and scaling law exponent. The PSRSIGSIM then shifts the profiles directly in time by the resulting delay, or convolves an exponential scattering tail with the input profiles chosen by a user-set flag within the function.

The TELESCOPE class encodes the properties of the desired telescope necessary to compute the radiometer noise and other observing-site specific effects. A user is 
able to supply telescope specifications, like the effective area and system temperature. Telescope systems can also be defined with specific BACKEND and RECEIVER classes. The RECEIVER class is currently primarily responsible for defining a bandpass response and calculating the radiometer noise. The BACKEND class is currently primarily used to inform on the maximum sampling rate of the telescope backend. As more features are added to the PSRSIGSIM, such as baseband signal simulation, more features may be added to the BACKEND class as well, such as simulating a polyphase filterbank. The PSRSIGSIM comes equipped with pre-defined Arecibo and Green Bank Telescope systems, but additional systems may be added to these, or a new telescope can easily be defined by the user.

The native output of the PSRSIGSIM is a simulated pulsar signal in the form of a NUMPY array (Van Der Walt et al., 2011). However, for this work output in the PSRFITS standard was needed in order for software downstream in the analysis pipeline, such as PSRCHIVE, to accept and process the files (Hotan et al., 2004; van Straten et al., 2012). To do this, we utilize the PUlSAR DATA TOOLBOX ${ }^{2}$ (PDAT) PYTHON package (Hazboun, 2020). While PDAT is not a part of the the PSRSIGSIM, we include an IO class in the PSRSIGSIM which contains a number of convenience functions. These use existing PSRFITS files as templates to make new files. Currently, the size of the data array within the template PSRFITs file is changed to match the size of the simulated data array, and subsequent metadata, such as the chosen value of DM, is also edited.

The PSRSIGSIM is designed to simulate one observing epoch of data at a time;

\footnotetext{
${ }^{2}$ https://github.com/Hazboun6/PulsarDataToolbox
} 
by iterating over sets of input parameters it is possible to produce phase-coherent data sets containing multiple observing epochs. This phase connection is performed by utilizing the PINT ${ }^{3}$ pulsar timing software (Luo et al., 2020) and an input pulsar ephemeris to replace the polynomial coefficient (POLYCO) values, which predict the pulsar's phase and period using polynomial expansion over a defined time period. We also note that no binary parameters or delays are currently included in any delay classes or in the creation of the POLYCOs. If a user desires to create a new PSRFITS file from scratch to contain the simulated data, this can be done with a number of currently existing software packages outside of the PSRSIGSIM such as PDAT (Hazboun, 2020), ASTROPY.IO.FITS ${ }^{4}$ (Astropy Collaboration et al., 2013; Price-Whelan et al., 2018), or FITSIO ${ }^{5}$.

More detailed descriptions as well as examples can be found on the READTHEDOCS $^{6}$ page of the PSRSIGSIM and in Hazboun et al. (2020).

\subsection{Methods}

Here we will describe the general methods used for making and analyzing our simulated data. The details of each set of simulated data appear in $\S 4.4$, while here we cover general processes used to produce the data and simulate each of the effects used. We discuss first the methods used for simulating the data with the PSRSIGSIM ${ }^{7}$ and then the methods used to obtain TOAs and fit the different timing parameters.

\footnotetext{
${ }^{3}$ https://github.com/nanograv/PINT

${ }^{4}$ https://docs.astropy.org/en/stable/io/fits/

${ }^{5}$ https://github.com/esheldon/fitsio

${ }^{6}$ https://psrsigsim.readthedocs.io/en/latest/readme.html

${ }^{7}$ PSRSIGSIM version 1.0 .0 is used throughout this work.
} 


\subsubsection{Generating Simulated Data}

All of our data are simulated using the PSRSIGSIM PYTHON package described in $§ 4.2$. For this work, we look at three different MSPs that are part of the NANOGrav pulsar timing array experiment (Arzoumanian et al., 2018c), PSRs J17441134, B1855+09, and B1953+29. These MSPs span a range of DMs, potentially allowing us to look at the covariances between DMX and FD parameters as a function of mean DM and/or number of FD parameters. Additionally they all have notable profile evolution (Alam et al., 2020c), rather long timing baselines (Arzoumanian et al., 2018c), and significant DM variations (Jones et al., 2017). For each simulation, we have a set of defined pulsar and observation parameters listed in Tables 4.1 and 4.2. These include the pulsar's name, period, DM, mean flux, spectral index, the desired bandwidth of the observation, number of frequency channels, center observing frequency, the observation length, and the telescope name. To simulate DM variations, we determine the individual DMX injected at each epoch using the trends from Jones et al. (2017), shown in Table 4.3. We use the DM reported in Table 4.1 as a reference DM where the injected DMX is zero. This reference DM is taken to be the value at the center epoch of the simulations, and when sinusoidal trends are added it is the value at phase zero. No additional noise is added to the predictions by these trends. If any other parameters are desired, such as FD parameters or scattering timescale $\left(\tau_{\mathrm{d}}\right)$, these may also be defined and used in the simulation.

We define a pulse shape to be input into the PSRSIGSIM for each observation 
depending on the simulated backend and receiver combination to mimic the standard timing procedure described in Demorest et al. (2013) and Arzoumanian et al. (2016). For this work, each set of pulse profiles is defined as a 2-D array in frequency and pulse phase, where we use 2048 phase bins. While the real NANOGrav observations record a different number of frequency channels depending on the receiver-backend combination, either 64,128 , or 512 , all are eventually folded down to 64 frequency channels (Arzoumanian et al., 2018c). As we can simulate any number of initial frequency channels, we simulated all of our initial observations with 64 frequency channels to avoid needless post-processing. For similar reasons, we also simulate all of our data with just a single subintegration of length equal to the total observing length. If no profile evolution with frequency is desired, we use the NANOGrav 11yr profile template defined at the appropriate center frequency for the pulse profile. This is input in the PSRSIGSIM as a 1-D array DATAPROFILE object, which is then tiled within the PSRSIGSIM so that the profile is the same in every frequency channel. An example is shown in the top panel of Figure 4.1.

When a 2-D array of frequency-dependent profiles is desired as the input into the PSRSIGSIM, we create them by starting with a post-processed, high signal-tonoise (S/N) NANOGrav observation with 64 frequency channel-depended profiles. This is done to be sure that all RFI has been removed and the data have been properly calibrated, though each observation was inspected by-eye to confirm this. We then smoothed this data using the PSRSMOOTH function of the PSRCHIVE data processing package (Hotan et al., 2004; van Straten et al., 2012). These smoothed profiles are then formatted into a 2-D PYTHON data array in frequency and pulse 
phase, as described above, using the PYPULSE ${ }^{8}$ PYTHON package (Lam, 2017). Only one set of model profiles was used for each receiver-backend combination. For example, if we simulate multiple observations at $1400 \mathrm{MHz}$, the noise-free profiles used at every simulated observing epoch will be the same, though the pulse shape may change with the observing frequency. However, the white noise that is added to the simulations will vary from epoch to epoch.

Since we have chosen to model our frequency-dependent profiles using real pulsar data for this work, we must also account for the effects of a pulsar's spectral index (e.g., Jankowski et al., 2018) and scintillation due to the ISM. Both of these effects are present in all pulsar observations, and if uncorrected, will change the pulse flux as a function of observing frequency in a non-user defined way. To remove these intrinsic effects, the pulse profiles were normalized such that all profiles have a peak flux of one in arbitrary flux units. However, to make our simulated data as realistic as possible a user-defined spectral index, reported in Table 4.1, is added back into the simulated data when the pulses are created. To do this, each normalized profile is multiplied by a frequency dependent constant such that

$$
S_{\text {mean }}(\nu)=S_{\text {mean }}\left(\nu_{\text {ref }}\right)\left(\frac{\nu}{\nu_{\text {ref }}}\right)^{\alpha} .
$$

Here $S_{\text {mean }}\left(\nu_{\text {ref }}\right)$ is the user-input mean flux referenced to some frequency, $\nu_{\text {ref }}, \nu$ is the center frequency of each frequency channel for each profile, $\alpha$ is the user input spectral index, and $S_{\text {mean }}(\nu)$ is the new mean flux of the spectral index adjusted

\footnotetext{
${ }^{8}$ https://github.com/mtlam/PyPulse
} 

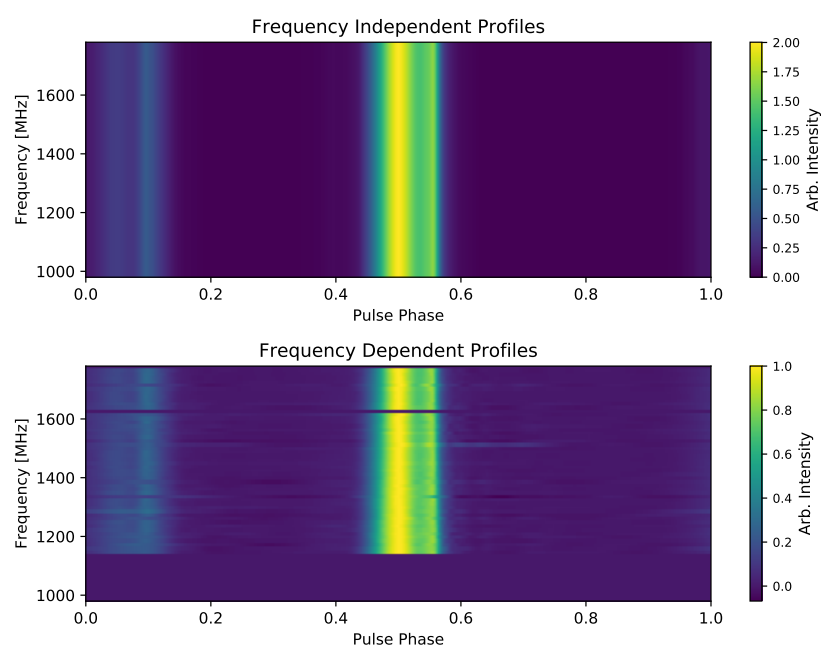

Figure 4.1 Injected pulse profiles for PSR B1855+09 used for this work. No additional scattering has been injected, and all peaks are normalized to an arbitrary intensity of one. Top: The same pulse profile, here the NANOGrav 11-yr profile for PSR B1955+09, is used for every frequency channel if no frequency-dependent pulse profile evolution is desired. Bottom: Frequency-dependent pulse profiles obtained from modeling a single real observation of PSR B1855+09. No apparent scintillation or spectral index effects remain after following the process detailed in $\S 4.3 .1$, and the frequency-dependent variations are clearly shown. Some channels have been removed due to RFI contamination.

profile at a frequency $\nu$.

Since our frequency-depended profiles were created from real, post-processed observations, profiles at some frequency channels had been removed due to contamination by radio frequency interference (RFI). Since we cannot realistically model profiles in the frequency channels that have been removed, we instead replace them with a profile of zeros. When creating TOAs from these profiles, all channels that were replaced with zeros in this way were removed as well, and are not included in any pulsar timing model fitting (described in $\S 4.3 .2$. This 2-D array of frequencydependent profiles is then input into the PSRSIGSIM as a DATAPORTRAIT object, and an example is shown in the bottom panel of Figure 4.1. We note these were all 
choices made for this work, and that the PSRSIGSIM is capable of using any set of 1- or 2-D user-generated pulse profiles.

While there is some inherent scatter broadening already contained within the real data used for our model frequency-dependent profiles, we do not know a priori how much the profiles have been scatter broadened, and hence can not separate this effect from intrinsic profile evolution. In some of our simulations (described in $\S 4.4$ ), however, we simulate pulse scatter broadening using the ISM class by defining a single input $\tau_{\mathrm{d}}$, referenced to an initial input frequency, for each simulated epoch. Within the PSRSIGSIM, $\tau_{\mathrm{d}}$ is scaled for each frequency channel as

$$
\tau_{\mathrm{d}_{\mathrm{i}}}=\tau_{\mathrm{d}}\left(\frac{\nu_{i}}{\nu_{\mathrm{ref}}}\right)^{\beta}
$$

Here $\nu_{\text {ref }}$ is the reference frequency of the input $\tau_{\mathrm{d}}, \nu_{i}$ is the center frequency of the $i$ th frequency channel, and $\beta$ is the scaling law exponent. The exponential scattering tail for each frequency channel is then calculated as $\exp \left(-t / \tau_{\mathrm{d}_{\mathrm{i}}}\right)$, where $t$ is the fractional time of each profile bin. The resulting frequency-dependent exponential scattering tails are then convolved with the pulse profiles. For our simulations, we assume a Kolmogorov medium, so $\beta=-4.4$, though $\beta$ can also be set by the user within the PSRSIGSIM. While it has been found that measurements of $\beta$ deviate from a Kolmogorov medium (e.g. Levin et al., 2016; Turner et al., 2020), we have chosen to use a constant value to minimize the number of variables that affect the covariance between DMX, FD, and $\tau_{\mathrm{d}}$. While studying how varying $\beta$ may affect these covariances is certainly of interest, this added complexity is beyond the scope 
of this work.

For this work, we have chosen to run simulations with both a single value of $\tau_{\mathrm{d}}$ across all epochs and with time-varying $\tau_{\mathrm{d}}$. In the case of a time-varying $\tau_{\mathrm{d}}$, we have chosen input values of $\tau_{\mathrm{d}}$ by randomly sampling a Gaussian distribution with mean and $1 \sigma$ variation reported in Table 4.1 and then taking the absolute value of the sampled $\tau_{\mathrm{d}}$. However, for PSR B1953+29, no RMS variation for $\tau_{\mathrm{d}}$ was reported by Levin et al. (2016), so we use a $1 \sigma$ variation of $20 \%$ the mean value. A different value of $\tau_{\mathrm{d}}$ is then input for every simulated epoch of observations. We note that because the PSRSIGSIM simulates just a single epoch at a time, a user may choose input values for $\tau_{\mathrm{d}}$ using any method.

After the pulses are simulated, they are dispersed with the ISM class. This is done by calculating the time delay due to dispersion,

$$
\Delta t_{\mathrm{DM}}=2.41 \times 10^{-4} \mathrm{~s}\left(\frac{\mathrm{DM}}{\mathrm{pc} \mathrm{cm}{ }^{-3}}\right)\left(\frac{\nu}{\mathrm{MHz}}\right)^{-2},
$$

in each frequency channel with respect to infinite frequency. Here $\nu$ is the center frequency of each frequency channel. The pulses are then shifted in Fourier space (Bracewell, 1999) to account for time shifts that are fractional sizes of the discrete time bins. For this work, the DM used is the sum of the base value reported in Table 4.1 and the individual DMX determined at each epoch as described above. However, we note that in general, the user may input any desired DM into the PSRSIGSIM.

Non-dispersive frequency-dependent time delays are also simulated. In particular, we directly shift the pulses in time to simulate the "FD" model for frequency- 
dependent pulse profiles. To do this, we calculate $\Delta t_{\mathrm{FD}}$ as (Zhu et al., 2015; Arzoumanian et al., 2016)

$$
\Delta t_{\mathrm{FD}}=\sum_{i=1}^{n} c_{i} \ln \left(\frac{\nu}{1 \mathrm{GHz}}\right)^{i}
$$

Here $c_{i}$ are the polynomial coefficients in time units, more often referred to as the FD parameters, such that $c_{1}=$ FD1 and so on, $n$ is the number of coefficients, and $\nu$ is the center frequency of each frequency channel. The pulses are then shifted in Fourier space as with $\Delta t_{\mathrm{DM}}$. Within the PSRSIGSIM the FD parameters are input in units of seconds. We report the number and value of each FD parameter used for each simulated pulsar in this work in Table 4.1, though in general the user may input any number of FD parameters with any value into the PSRSIGSIM.

Once these delays are added, we then define the TELESCOPE used in this work as either the 305-m William E. Gordon Telescope of the Arecibo Observatory or the 100-m Green Bank Telescope of the Green Bank Observatory. We do this using the default ARECIBO or GBT definition in the PSRSIGSIM, though a user may define any telescope system they wish for their own simulations. Radiometer noise is then added to the simulated data based on the desired receiver-backend configuration. The noise is sampled from a chi-squared distribution with a number of degrees of freedom equal to the number of single pulses in each subintegration. This is then multiplied by the noise variance $\left(\sigma_{S}\right)$, calculated as defined in Lorimer \& Kramer (2004),

$$
\sigma_{S}=\frac{\mathrm{T}_{\text {sys }}+\mathrm{T}_{\text {sky }}}{G \sqrt{n_{\mathrm{p}} d t \mathrm{BW}_{\mathrm{chan}}}},
$$

where $\mathrm{T}_{\text {sys }}$ is the system temperature, $\mathrm{T}_{\text {sky }}$ is the sky temperature, $G$ is the tele- 
scope gain, $n_{\mathrm{p}}$ is the number of polarizations, $d t$ is the length of each phase bin or $1 /$ (sample rate), and $\mathrm{BW}_{\text {chan }}$ is the bandwidth of a frequency channel. Currently the simulator does not model the sky temperature, so we take $T_{\text {sky }}=0$ for all simulations. Since only total intensity signals are supported at this time, we assume that the total intensity is the sum of two intensities and so $n_{\mathrm{p}}=2$ for all simulations.

Since the user input profiles are normalized within the PSRSIGSIM, as they may be input with arbitrary units, this is then scaled by the maximum flux, $S_{\max }$, calculated from the mean flux,

$$
S_{\max }=\frac{S_{\text {mean }} n_{\text {bins }}}{\sum_{i=1}^{n_{\text {bins }}} p_{i}} .
$$

Here $n_{\text {bins }}$ are the number of phase bins per profile (2048 in all of the simulations in this work), and $p_{i}$ is the intensity of the model profile at the $i$ th phase bin. If using frequency-dependent pulse profiles, the profile with the maximum integrated flux (in arbitrary units) is used. This radiometer noise is then further scaled by a normalization coefficient, $U_{\text {scale, }}$ since, as mentioned above, the model profiles are normalized within the PULSAR class. This constant is calculated as defined in Lam (2018)

$$
U_{\text {scale }}=\frac{1.0}{\left(\sum_{i=1}^{n_{\text {bins }}} p_{i}\right) / n_{\text {bins }}},
$$

where again the profile used is from the frequency channel that results in the maximum integrated flux.

The final simulated data are contained within a NUMPY array (Van Der Walt 
et al., 2011). However, for this work, since we require the use of the PSRCHIVE software, we have used the convenience functions provided in the IO class and described in $§ 4.2$ to save the full simulated data array as a PSRFITS file (Hotan et al., 2004) as described in $\S 4.2$.

\subsubsection{TOAs and Residuals}

Once the data have been simulated in PSRFITS file format, they are analyzed with both PSRCHIVE and $\mathrm{PINT}^{9}$. The data are simulated such that all observations match the post-processed NANOGrav standard timing methods (Demorest, 2018), with a single subintegration and 64 frequency channels. For simulated Arecibo data, this results in frequency channels with widths of 1.5625 and $12.5 \mathrm{MHz}$ at 430 and $1400 \mathrm{MHz}$, respectively. For simulated GBT data, this results in frequency channels with widths of 3.125 and $12.5 \mathrm{MHz}$ at 820 and $1400 \mathrm{MHz}$, respectively.

TOAs are obtained from the simulated data with the PAT function in PSRCHIVE. We use the corresponding NANOGrav 11-yr pulse profile templates for the template matching process. This method employs a constant template profile at the appropriate frequency bands regardless of whether frequency-dependent profiles were used in the simulations to better match the standard template-fitting methods used by NANOGrav (Taylor, 1992; Demorest, 2018).

Normally, certain frequency channels are ignored in the NANOGrav timing pipeline as they are highly contaminated by RFI (Arzoumanian et al., 2016; Demorest, 2018). While we generate no RFI in our simulated data, we mimic this loss in

\footnotetext{
${ }^{9}$ PINT version 0.7.0 is used throughout this work.
} 
sensitivity by removing all TOAs from these ranges in all simulation analyses. This includes channels where no frequency-dependent profile model has been generated, as described above and shown in Figure 4.1. For simulated Arecibo data, the removed ranges are 380-423, 442-480, 980-1150, and 1618-1630 MHz. For simulated GBT data, the removed ranges are 794.6-798.6, 814.1-820.7, 1100-1150, 1250-1262, 1288-1300, 1370-1385, 1442-1447, 1525-1558, 1575-1577, 1615-1630 MHz.

We then calculate the timing residuals using the PINT pulsar timing package (Luo et al., 2020). Each pulsar timing model is extremely simple and includes only the position, period, DM, DMX, the number of FD parameters equal to that listed in Arzoumanian et al. (2018c), and one JUMP parameter to account for unmodeled profile evolution and other effects between the low and high frequency simulated data. Of these we fit only combinations of DMX, FD, and JUMP parameters, holding all other values fixed. Since we have not included any motions of the Earth, we assume that all TOAs that we have obtained are already barycentered. We do not include any effects such as parallax, proper motion, or binary motion and therefore do not fit for these in our timing model.

When fitting the different DMX values for each simulation, we follow Arzoumanian et al. (2016) and Arzoumanian et al. (2018c) and bin our simulated TOAs in groups of 15 days for simulated epochs before MJD 56000, and 6 days after MJD 56000. The adjustment in binning comes from the less frequent observations that occurred early on in the NANOGrav timing program (Arzoumanian et al., 2018c). We then fit our timing model parameters using the generalized least squares fitter in PINT and compare the fit values of DMX and the FD parameters, 
which we will denote as $\widehat{\mathrm{DMX}}$ and $\widehat{\mathrm{FD}}$, to the injected values.

\subsection{Simulated Data}

The parameters that were used to make the simulated data for PSRs J17441134, B1855+09, and B1953+29 are shown in Table 4.1. We note that while real pulsars have many additional timing parameters (e.g., spin down, proper motions, etc.), we do not simulate these effects in any of our data sets presented here. Our simulated data therefore represent barycentered observations that have had all nonfrequency-dependent delays removed ${ }^{10}$.

The simulated data sets for each pulsar are split into two sets of simulations. The first set consists of simulations where the pulse profile is frequency-independent for a given observing band. The recovered parameters generally match the injected parameters in this set and are used primarily for comparison. The second set uses realistic, frequency-dependent pulse profiles as described in $§ 4.3 .1$. In total, we simulated nine different data sets with different injections for each pulsar. Five of them used frequency-independent pulse profiles for comparison purposes, and the other four were used to analyze the covariances between the frequency-dependent parameters. The basic injections and values used for each simulation can be found in Table 4.4 .

All simulations span the same length as the observations of each pulsar using the NANOGrav observing epochs from Arzoumanian et al. (2018c). While it is

\footnotetext{
${ }^{10}$ The inclusion of additional timing parameters and the covariances between them is generally of interest and is a topic for future work.
} 
Table 4.1 Simulated Pulsar Parameters

\begin{tabular}{cccc}
\hline \hline Parameter & J1744-1134 & B1855+09 & B1953+29 \\
\hline Period $(\mathrm{ms})$ & 4.075 & 5.362 & 6.133 \\
DM $\left(\mathrm{pc} \mathrm{cm} \mathrm{cm}^{-3}\right)$ & 3.09 & 13.30 & 104.5 \\
FD1 $(\mu \mathrm{s})$ & $-383.4 \pm 88.5$ & $128.7 \pm 24$ & $139.5 \pm 7.8$ \\
FD2 $(\mu \mathrm{s})$ & $395.6 \pm 89.5$ & $-147.5 \pm 29$ & $-61.0 \pm 7.8$ \\
FD3 $(\mu \mathrm{s})$ & $-241.1 \pm 60.2$ & $81.6 \pm 18$ & - \\
FD4 $(\mu \mathrm{s})$ & $98.9 \pm 23.3$ & - & - \\
$S_{430}(\mathrm{mJy})$ & - & 14.56 & 10.77 \\
$S_{820}(\mathrm{mJy})$ & 2.93 & - & - \\
$S_{1400}(\mathrm{mJy})$ & 0.98 & 2.13 & 0.69 \\
$\alpha$ & -1.77 & -1.45 & -2.16 \\
$\tau_{\mathrm{d}}(\mathrm{ns})$ & $3.3 \pm 1.6$ & $8.1 \pm 4.4$ & $55.3( \pm 11.1)$ \\
\hline
\end{tabular}

Note. - Parameters describing the three MSPs that were used in these simulations. Period, DM, and FD1-4 values are from the NANOGrav 11-yr data set (Arzoumanian et al., 2018c). Flux values $(S)$ and spectral index $(\alpha)$ values are from Alam et al. (2020b). Scattering timescales $\tau_{\mathrm{d}}$ are all referenced to $1500 \mathrm{MHz}$ and for PSRs J1744-1134 and B1855+09 come from Turner et al. in prep, and for PSR B1953+29 from Levin et al. (2016). All uncertainties are $1 \sigma$, with the uncertainties on scattering delay defined as the RMS variation over the data set. No variation on $\tau_{\mathrm{d}}$ for PSR B1953+29 was reported in Levin et al. (2016) so we define it to be $20 \%$ the measured value. 
not necessary to simulate a full data set like this to explore the covariances between these parameters, we do this in part to demonstrate that the PSRSIGSIM can simulate long sets of unevenly samples observing epochs while maintaining a precise phase connection. In addition, it demonstrates the efficiency of the PSRSIGSIM, as the total time for each of the nine simulations to run for the two longer data sets, those of PSRs J1744-1134 and B1855+09, was 5 minutes on an Intel(R) Xeon(R) CPU E5-2630 0 @ 2.30GHz with 24 processors and 64 GB of RAM. In addition to using realistic observing epochs as a benchmarking test for the PSRSIGSIM, having many DMX bins better quantifies the variations that may be seen in DMX due to profile evolution or scatter broadening. Finally, as the FD parameters are fit globally over the entire data set, their fit values are sensitive to the length and number of observations in the simulated data set.

For simulated observations using the Arecibo telescope, we simulate only data from the PUPPI backend (Ford et al., 2010) and for observations simulated using the GBT, we simulate only data using the GUPPI backend (DuPlain et al., 2008). While much of the early observations of these MSPs were done with the GASP or ASP backend (Demorest, 2007), there are additional systematics introduced into the pulsar timing when switching between backends that is beyond the scope of this work. The observing frequencies of each pulsar, either $430 \mathrm{MHz}$ or $1400 \mathrm{MHz}$ at Arecibo or $820 \mathrm{MHz}$ or $1400 \mathrm{MHz}$ at the GBT, are the same as in Arzoumanian et al. (2018c). The parameters for each receiver-backend combination are reported in Table 4.2. The observation lengths, the time of each simulated observing epoch, come from the length of the actual observation that was used to generate the frequency- 
dependent pulse profiles. These lengths represent a typical observing length for each pulsar as observed by NANOGrav, though they are kept constant for each simulated observing epoch.

\subsubsection{Frequency-Independent Pulse Profile Simulations}

The first simulation in this set was the "No Variation" simulation. Every simulated epoch used a constant value of DM (e.g., all DMX values were zero), and included no additional time delays (e.g., from FD parameters or scatter broadening). The purpose of this was to test the simplest case simulation and provide a baseline to compare to other simulations with addition injected effects. For each observation we determine $\triangle \mathrm{DM}$, the recovered DMX minus the injected $\mathrm{DMX}, \Delta \mathrm{DM}=\widehat{\mathrm{DMX}}-$ DMX. This is shown in red at every simulated observing epoch for all three simulated pulsars in Figure 4.2. We note that the $\widehat{\mathrm{DMX}}$ values have been mean subtracted as $\left(\widehat{\mathrm{DMX}}_{i}-\langle\widehat{\mathrm{DMX}}\rangle\right)$ for each DMX epoch $i$, and the error bars shown represent the errors on the mean subtracted value. This is done because it allows us to separate the uncertainty of each DMX measurement from that of the mean DM, since there is a large covariance between these parameters (Arzoumanian et al., 2016, 2018c).

We similarly determine $\Delta \mathrm{FD}$ parameters, the recovered FD parameter minus injected $\mathrm{FD}$ parameter, $\Delta \mathrm{FD}_{\mathrm{i}}=\widehat{\mathrm{FD}_{\mathrm{i}}}-\mathrm{FD}_{i}$, for each individual FD parameter i. For this simulation, these shown in red in the two upper panels of Figures 4.3, 4.4, and 4.5 for each pulsar respectively. All of the recovered $\Delta \mathrm{DM}$ and $\Delta \mathrm{FD}$ parameters for these simulations are shown in the same panels of the same Figures, though with 


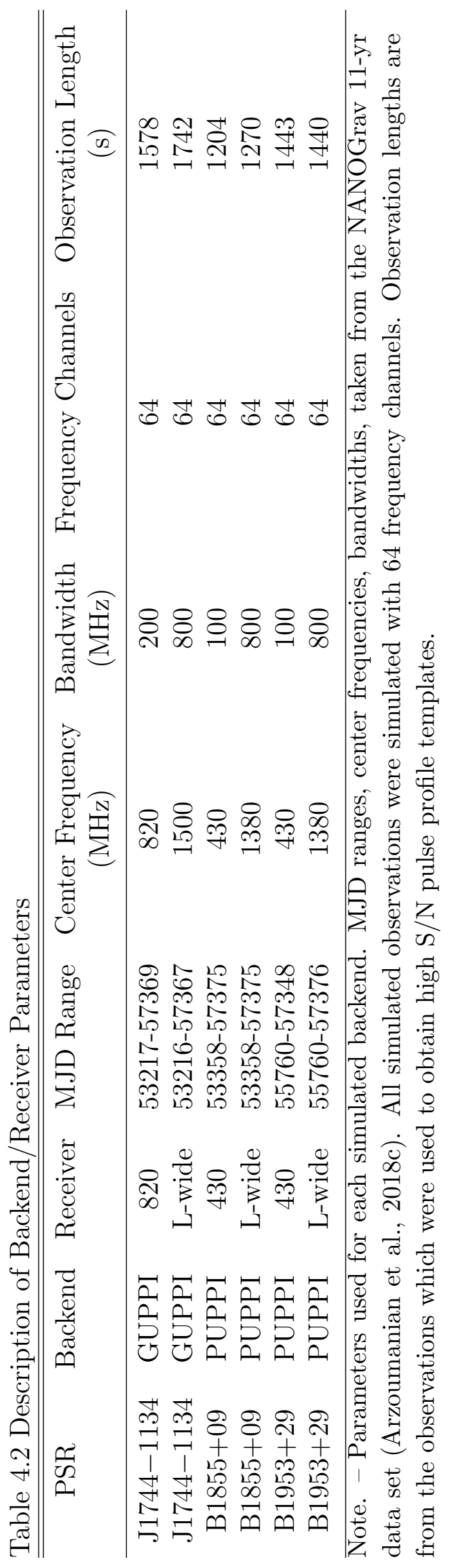


Table 4.3 DM Variation Parameters

\begin{tabular}{cccc}
\hline \hline PSR & $\begin{array}{c}\text { DM Slope } \\
\left(10^{-3} \mathrm{pc} \mathrm{cm}^{-3} \mathrm{yr}^{-1}\right)\end{array}$ & $\begin{array}{c}\text { DM Amplitude } \\
\left(10^{-4} \mathrm{pc} \mathrm{cm}^{-3}\right)\end{array}$ & $\begin{array}{c}\text { DM Period } \\
(\text { days })\end{array}$ \\
\hline J1744-1134 & -0.069 & 0.4 & 383 \\
B1855+09 & 0.382 & 0.5 & 364 \\
B1953+29 & -1.3 & 3.0 & 356 \\
\hline
\end{tabular}

Note. - Slope, amplitude, and period of the DM variations used for each pulsar that was simulated as derived by Jones et al. (2017).

different colors. All recovered values, $\widehat{\mathrm{DMX}}$ and $\widehat{\mathrm{FD}}$, shown in these figures were determined by fitting for all parameters: DMX, all FD parameters, and a single JUMP parameter.

The second simulation is the "DM Variations" simulation. Here the total injected DM is the initial value given in Table 4.1 plus a small variation added based on the parameters given in Table 4.3. The variations for all simulated pulsars had both a linear and sinusoidal trend with slope, amplitude, and period as determined by Jones et al. (2017). The resulting $\Delta \mathrm{DM}$ and $\Delta \mathrm{FD}$ parameters, similar to that shown for the "No Variations" simulation, are shown in purple.

The third simulation in this set is the "FD Injection." While the physical process that FD parameters describe is mainly attributed to pulse profile evolution in frequency (Zhu et al., 2015), they define a time delay directly given by Eq. 4.4. To provide a baseline for recovering the injected FD parameters, we directly shift the simulated pulses in time based on the FD parameters listed in Table 4.1. We do this instead of varying the profiles directly because we do not know a priori what the shifts due to profile evolution are, we can only fit them empirically. The resulting 


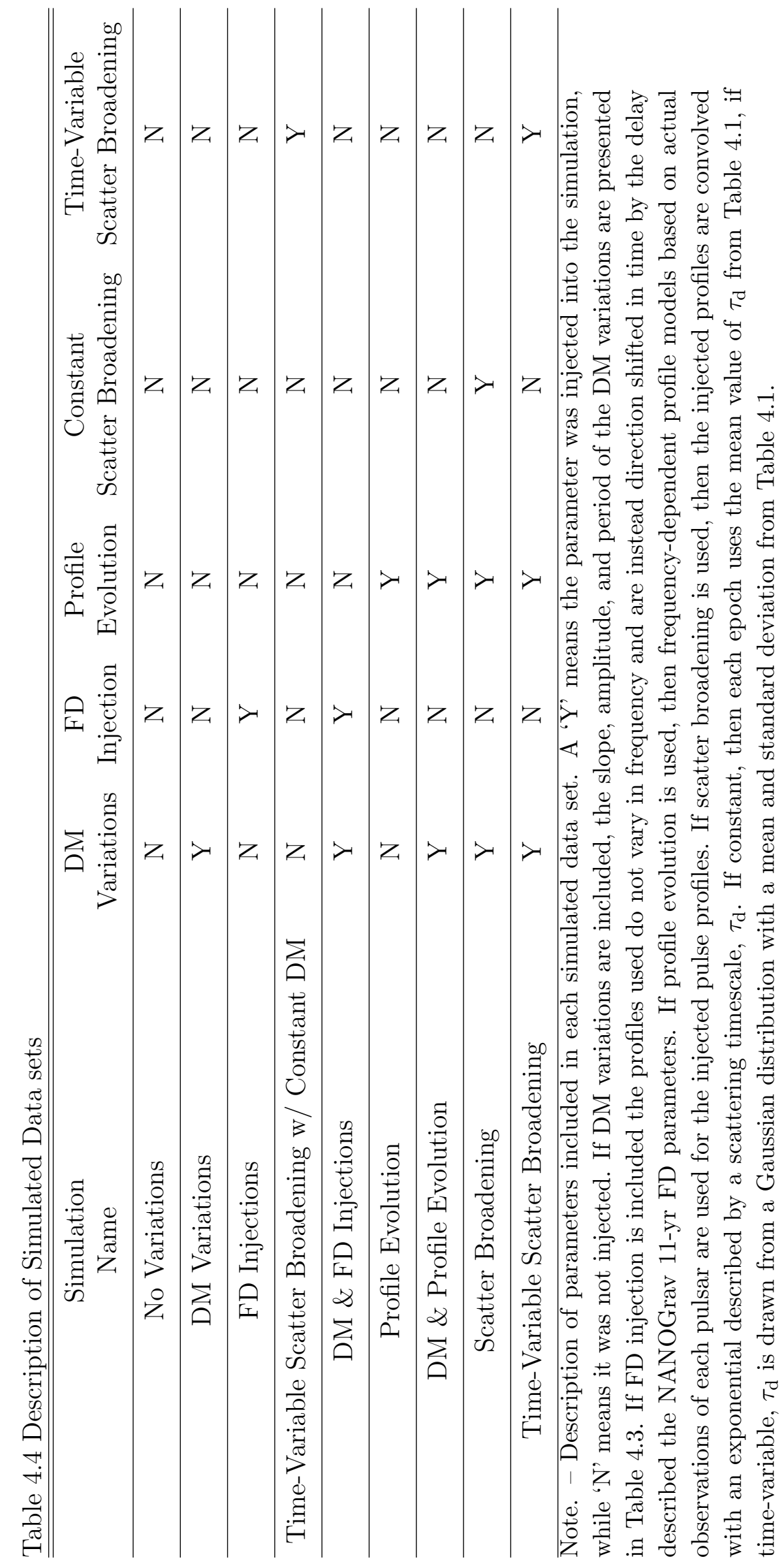


$\triangle \mathrm{DM}$ and $\triangle \mathrm{FD}$ parameters for this simulation are shown in orange.

The fourth simulation here is "Time-Variable Scattering with Constant DM." Here we again use a constant value of DM, but also inject time-variable values of $\tau_{\mathrm{d}}$ on a per-epoch basis, selected as described in $\S 4.3 .1$. This gives us baseline to compare how $\widehat{\mathrm{DMX}}$ is affected by this time-variable scattering in more complex simulations. The resulting $\triangle \mathrm{DM}$ and $\Delta \mathrm{FD}$ parameters for this simulation are shown in light green.

Our final initial simulation, "DM \& FD Injections" is a combination of the second and third initial simulations. This was done to provide a baseline for the accuracy of $\widehat{\mathrm{DMX}}$ and $\widehat{\mathrm{FD}}$ since they are both dependent on the emission frequency. The resulting $\triangle \mathrm{DM}$ and $\triangle \mathrm{FD}$ parameters for this simulation are shown in light blue.

While all of the recovered values shown in Figures 4.2, 4.3, 4.4, and 4.5 come from fitting for all parameters, DMX, all FD parameters, and a JUMP, we also fit each of these simulations using just a single JUMP, just DMX and a single JUMP, and just all applicable FD parameters and a single JUMP (for a total of four different fits for each simulation). We report the RMS of the timing residuals $\left(\mathcal{R}_{\text {rms }}\right)$, reduced chi-squared $\left(\chi_{\mathrm{r}}^{2}\right.$ of the fit timing model, the RMS of the $\Delta \mathrm{DM}$ values $(\widehat{\mathrm{DMX}}-\mathrm{DMX})$, $\Delta \mathrm{DM}_{\mathrm{rms}}$, the RMS of the $\Delta \mathrm{FD}$ parameters $\left(\widehat{\mathrm{FD}_{\mathrm{i}}}-\mathrm{FD}_{\mathrm{i}}\right), \Delta \mathrm{FD}_{\mathrm{rms}}$, and the fit value of the JUMP, for each of these fits per simulation per pulsar in Tables 4.5, 4.6, 4.7, $4.8,4.9$, and 4.10 respectively. 


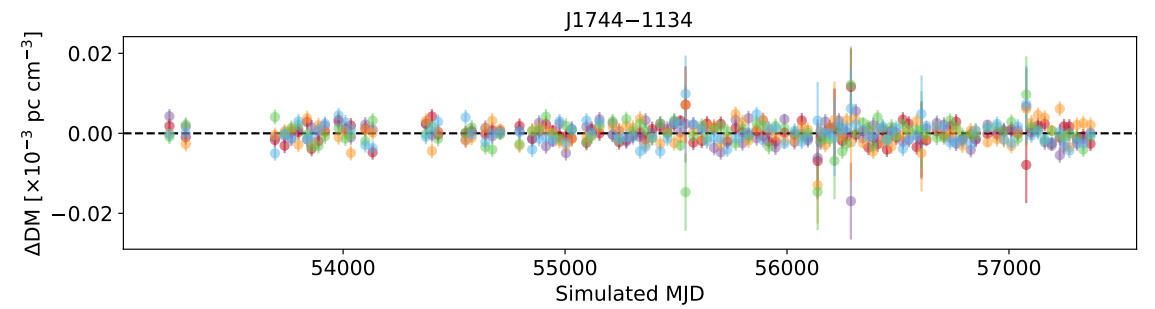

No Variation

$\phi$ DM Variations

FD Injection

Time-Variable

Scattering w/

Constant DM

DM \& FD Variations

B1855+09

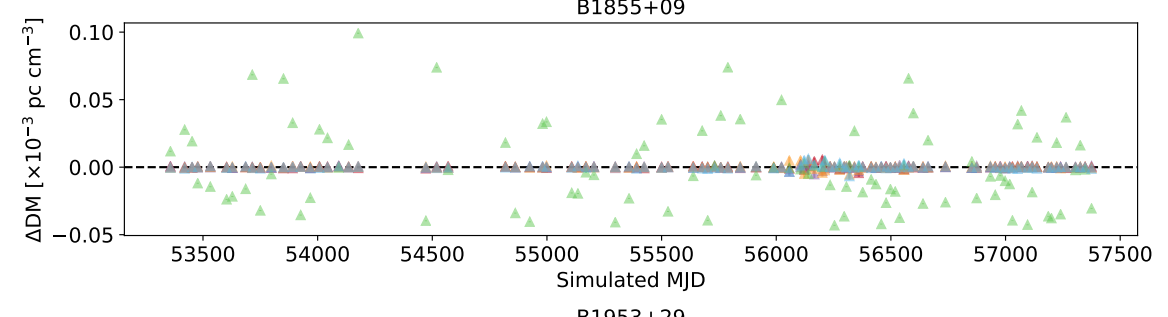

No Variation

DM Variations

FD Injection

Time-Variable

Scattering w/

Constant DM

DM \& FD Variations

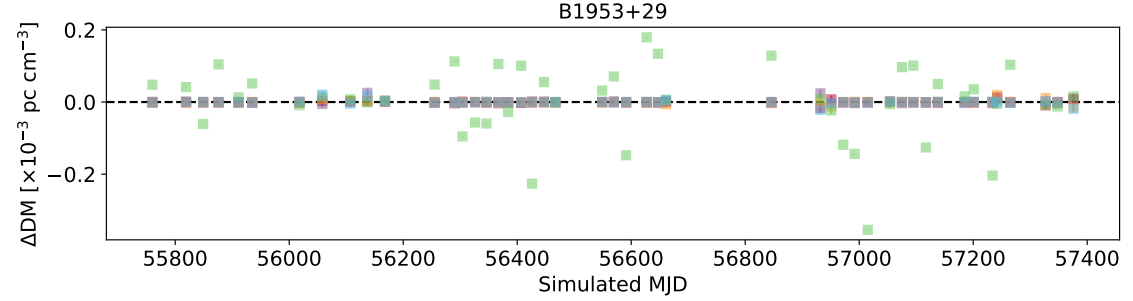

中 No Variation

申 DM Variations

中 FD Injection

Time-Variable

Scattering w/

Constant DM

1. DM \& FD Variations

Figure 4.2 Resulting $\Delta \mathrm{DM}$ values for all three simulated pulsars for simulations where no frequency-dependent pulse profiles were used (described in §4.4.1) when fitting for DMX, all FD parameters, and a JUMP. Different symbols are used for each pulsar. The black dashed lines represent the zero lines. All points for each pulsar and each simulation are scattered around this zero line, showing that they are being appropriately recovered and fit for. 

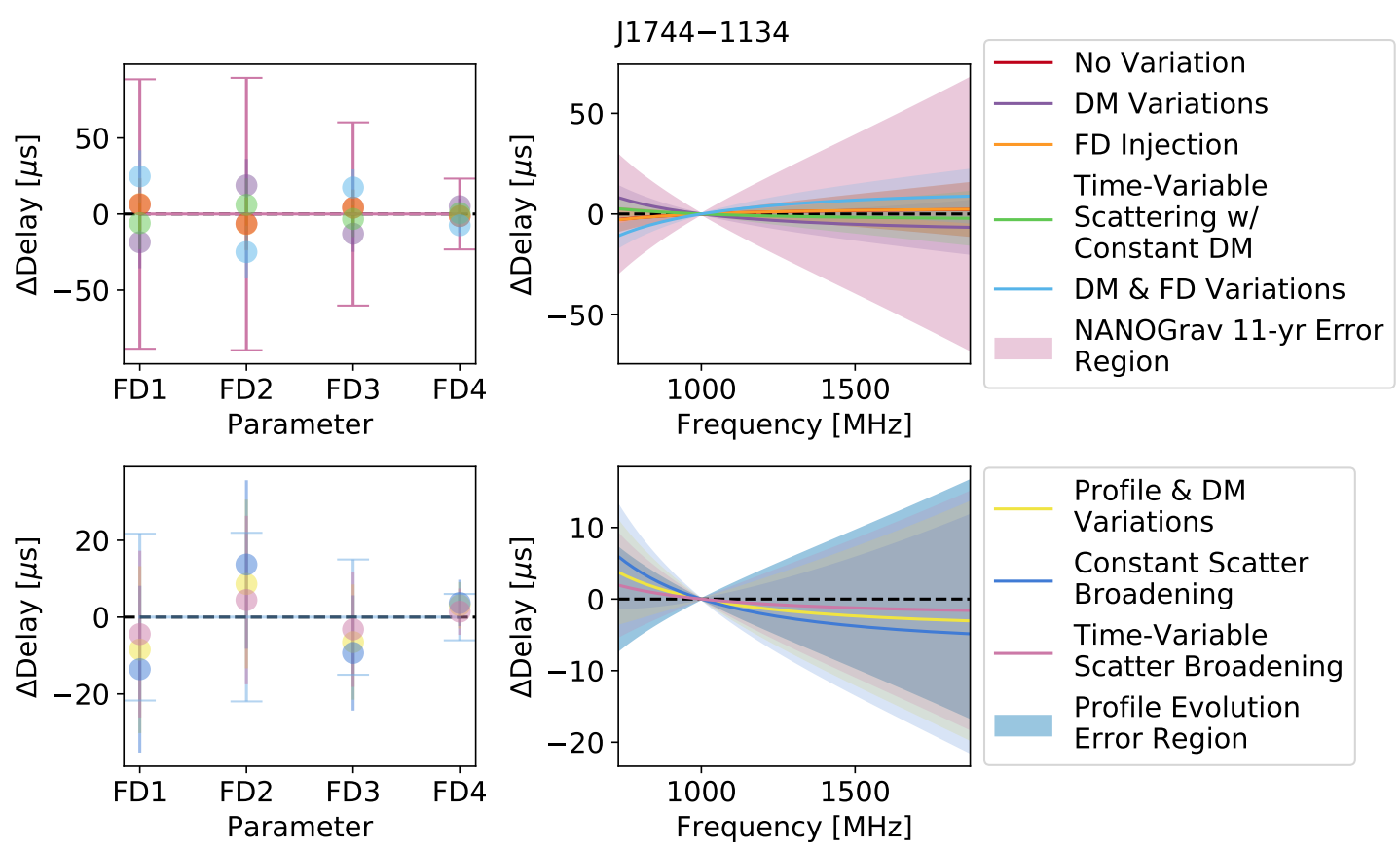

Figure 4.3 Upper Left: Resulting $\Delta \mathrm{FD}$ parameters for five different simulated data sets of eleven years where no frequency-dependent pulse profiles were used for PSR J1744-1134 (described in §4.4.1). All recovered values come from fitting for DMX, all FD parameters, and a JUMP. The recovered parameters match well with the injected parameters. Upper Right: Same as upper left but showing the delay curve across frequency space defined by the $\Delta \mathrm{FD}$ parameters shown in the upper left plot. Shaded regions represent the $1 \sigma$ recovered errors. Lower Left: Same as upper left, but for four different simulations where frequency-dependent pulse profiles were used (described in §4.4.2). Lower Right: Same as upper right, but for the simulations listed in the lower left.

\subsubsection{Frequency-Dependent Pulse Profile Simulations}

Next we use a different set of frequency-dependent profiles, one for each different receiver-backend combination for each pulsar, is used as described in §4.3. As noted in $§ 4.3 .1$, we use only one set of frequency-dependent profiles for each receiver-backend combination. Since there are no variations in the profile evolution in time, e.g., due to scintillation (Cordes, 1986), we do not expect to recover exactly the same FD parameters as reported in Table 4.1. We do, however, expect similar 

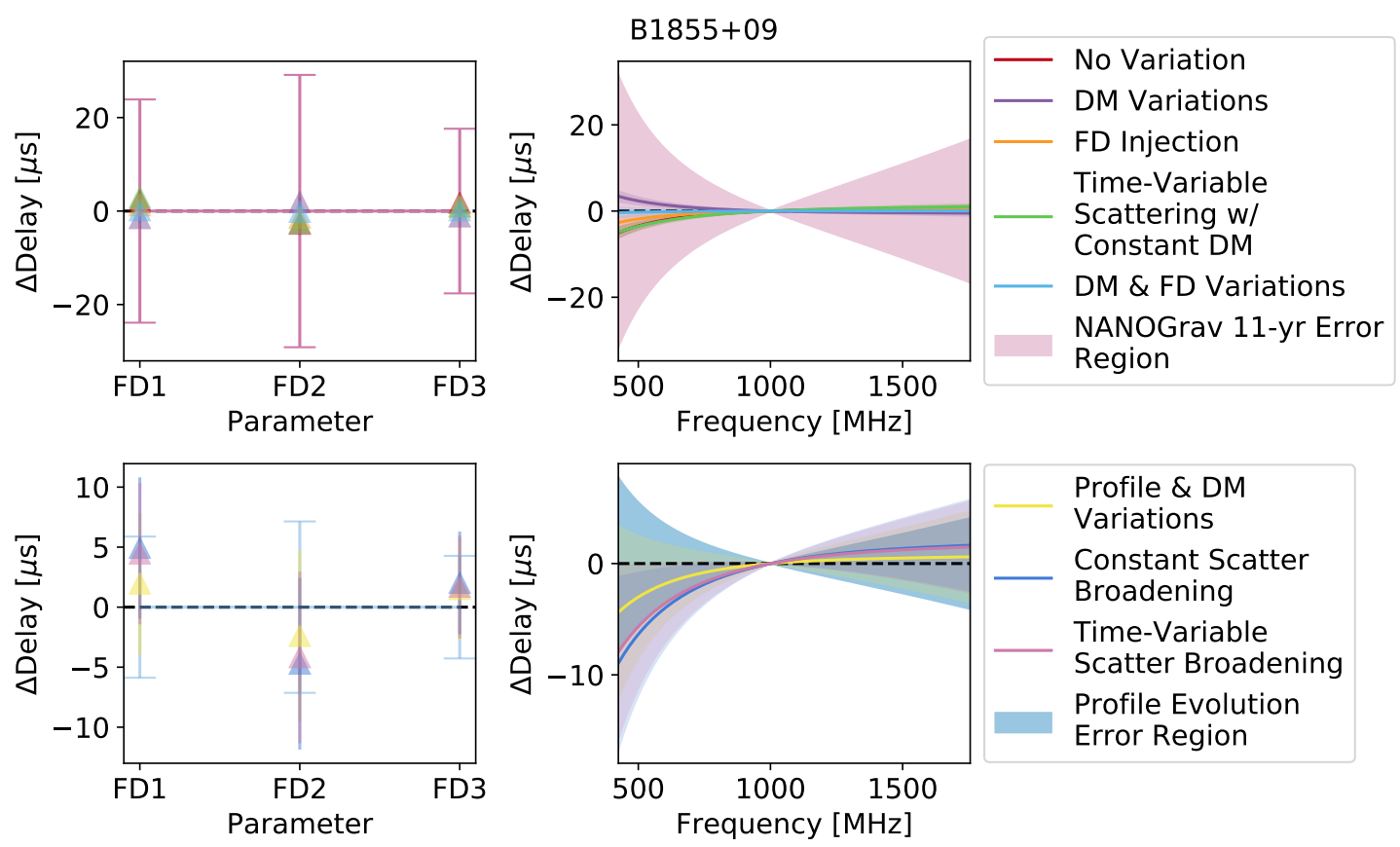

Figure 4.4 Upper Left: Resulting $\Delta \mathrm{FD}$ parameters for five different simulated data sets of eleven years where no frequency-dependent pulse profiles were used for PSR B1855+09 (described in §4.4.1). All recovered values come from fitting for DMX, all FD parameters, and a JUMP. The recovered parameters match well with the injected parameters. Upper Right: Same as upper left but showing the delay curve across frequency space defined by the $\Delta$ FD parameters shown in the upper left plot. Shaded regions represent the $1 \sigma$ recovered errors. Lower Left: Same as upper left, but for four different simulations where frequency-dependent pulse profiles were used (described in §4.4.2). Lower Right: Same as upper right, but for the simulations listed in the lower left.

FD parameters, with the same signs and orders of magnitude.

To determine what the contribution of the frequency-dependent profiles is to the FD parameters, our first simulation in this set, labeled "Profile Evolution", uses a constant DM such that all injected DMX values are zero, and includes only the frequency-dependent profile. This allows us to determine what the expected contribution of the chosen set of frequency-dependent profiles is, and help to quantify any deviations in $\widehat{\mathrm{FD}}$ as more frequency-dependent effects are added. The resulting $\Delta \mathrm{DM}$, similar to that shown for the previous set of simulations, are shown in blue 

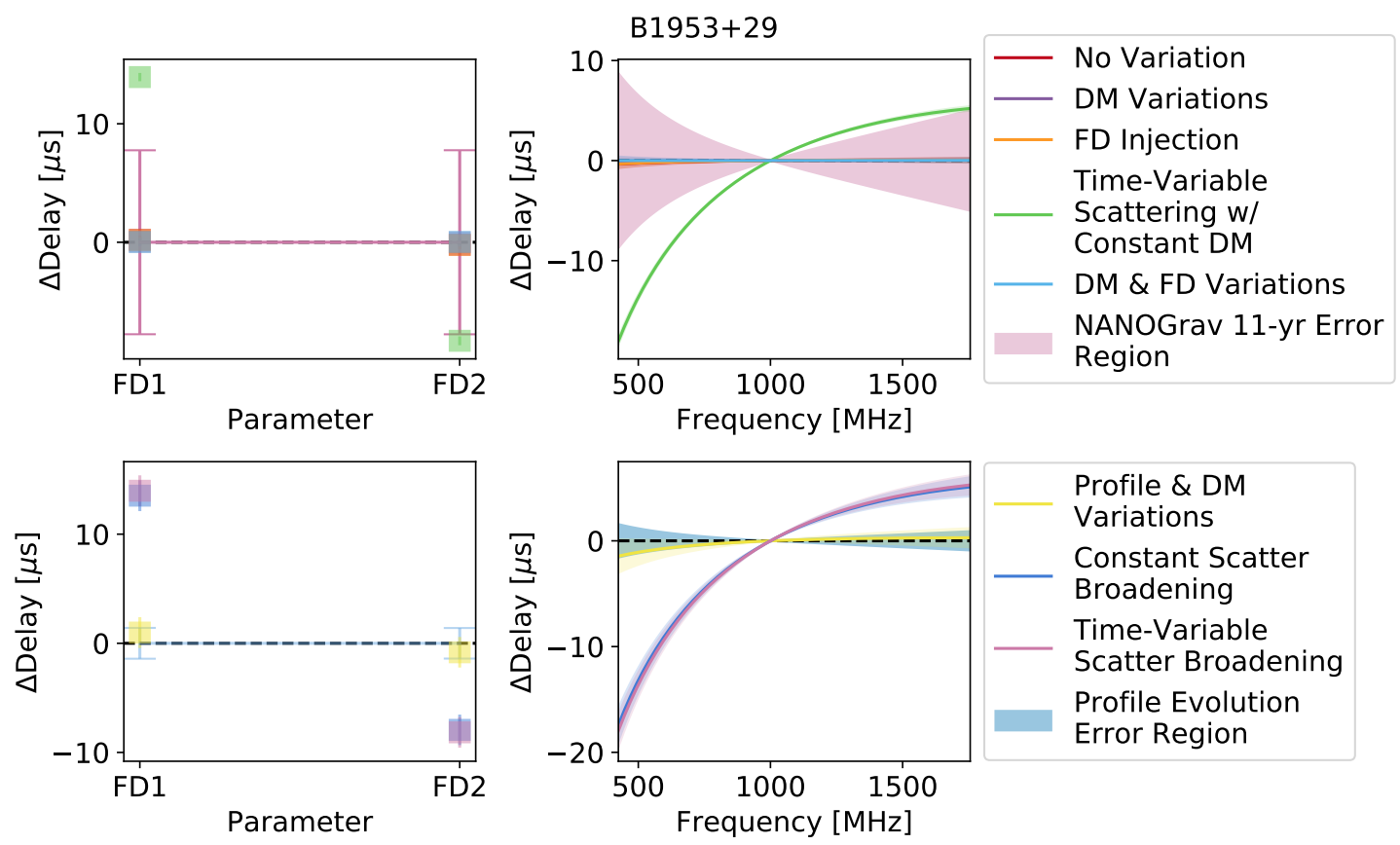

Figure 4.5 Upper Left: Resulting $\Delta \mathrm{FD}$ parameters for five different simulated data sets of eleven years where no frequency-dependent pulse profiles were used for PSR B1953+29 (described in §4.4.1). All recovered values come from fitting for DMX, all FD parameters, and a JUMP. The recovered parameters match well with the injected parameters. Upper Right: Same as upper left but showing the delay curve across frequency space defined by the $\Delta$ FD parameters shown in the upper left plot. Shaded regions represent the $1 \sigma$ recovered errors. Lower Left: Same as upper left, but for four different simulations where frequency-dependent pulse profiles were used (described in §4.4.2). Lower Right: Same as upper right, but for the simulations listed in the lower left.

in Figure 4.6 and the resulting $\Delta \mathrm{FD}$ parameters for this simulation, also in blue, are shown in the two lower panels of Figures 4.3, 4.4, and 4.5 for each pulsar respectively. All values of $\Delta \mathrm{DM}$ and $\Delta \mathrm{FD}$ parameters for these simulations are shown in the same panels of the same Figures, though with different colors. The resulting $\Delta \mathrm{FD}$ parameters for this simulation have values of zero with an associated error bar, since we do not know their value a priori. For this simulation $\widehat{\mathrm{FD}}$ are used as the baseline for all other simulations in this set.

The second simulation, "DM \& Profile Evolution", uses both the frequency- 


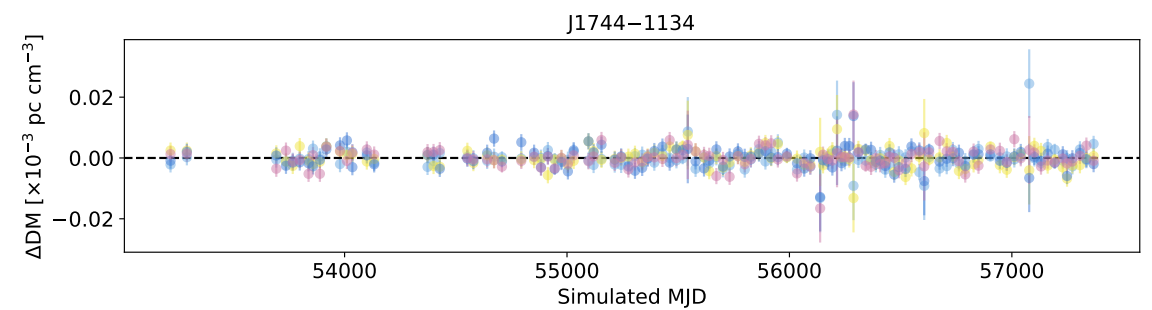

$\phi \quad$ Profile Evolution Profile \& DM Variations

Constant Scatter

Broadening

Time-Variable

Scatter Broadening

B1855+09

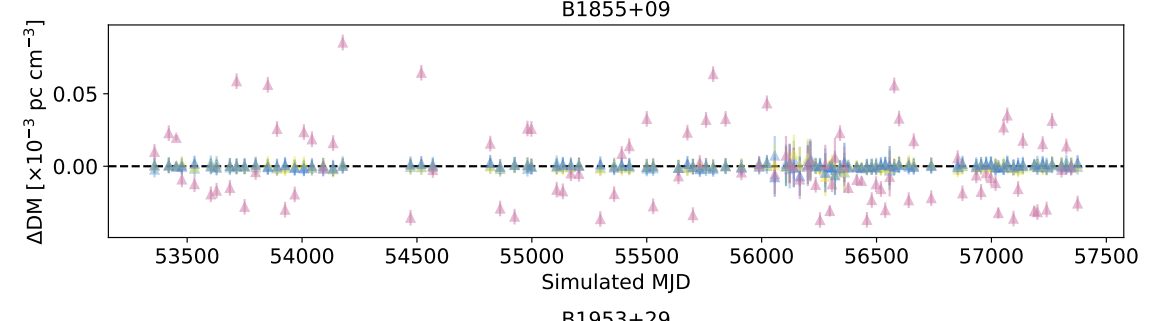

4 Profile Evolution Profile \& DM Variations

Constant Scatter Broadening Time-Variable

Scatter Broadening

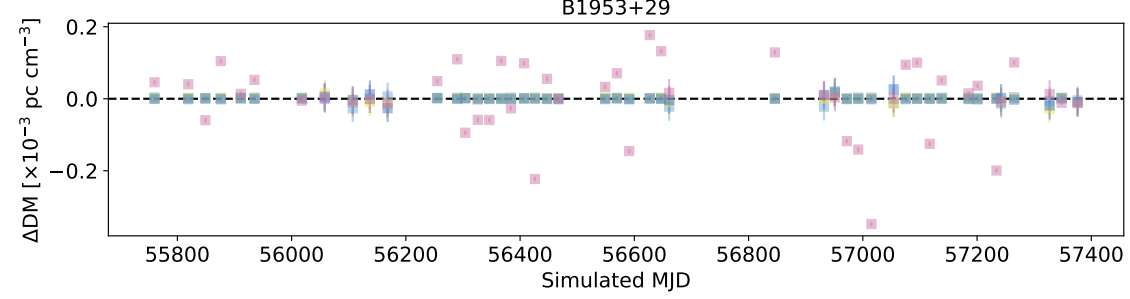

Profile Evolution Profile \& DM Variations Constant Scatter Broadening Time-Variable Scatter Broadening

Figure 4.6 Resulting $\Delta \mathrm{DM}$ values for all three simulated pulsars for simulations where frequency-dependent profile evolution was modeled (described in §4.4.2) when fitting for DMX, all FD parameters, and a JUMP. Different symbols are used for each pulsar. The black dashed lines represent the zero lines. All points for each pulsar and each simulation are scattered around this zero line, showing that they are being appropriately recovered and fit for. 
dependent profiles as well as the DM variations that were used in the initial simulations described in $\S 4.4 .1$. This simulation allows us to explore the covariances between DMX and profile evolution, and compare them to the covariances when FD parameters are directly injected via time shift. The resulting $\Delta \mathrm{DM}$ and $\Delta \mathrm{FD}$ parameters for this simulation are shown in yellow.

The third simulation, "Scatter Broadening," is the same as "DM \& Profile Evolution" but here the frequency-dependent profiles have been convolved with an exponential defined by a single mean scattering time scale, given in Table 4.1. As pulse scatter broadening is also a frequency-dependent effect, we expect it to have some small effect on $\widehat{\mathrm{DMX}}$ and $\widehat{\mathrm{FD}}$ (Rickett, 1977; Levin et al., 2016). However, since for this simulation only a constant value of $\tau_{\mathrm{d}}$ is injected, we expect the FD parameters to account for most, if not all of this variation (Zhu et al., 2015; Arzoumanian et al., 2016). The resulting $\Delta \mathrm{DM}$ and $\Delta \mathrm{FD}$ parameters for this simulation are shown in dark blue.

The final simulation of this set, "Time-Variable Scatter Broadening", is the same as "Scatter Broadening" but here we have randomly sampled values of $\tau_{\mathrm{d}}$ to be injected at each epoch as described in $§ 4.3 .1$. This simulation represents the most realistic of our simulations. Since here $\tau_{\mathrm{d}}$ changes, we expect $\widehat{\mathrm{DMX}}$ to be effected more substantially as the FD parameters are fit over the entire data set, not epoch to epoch. The resulting $\Delta \mathrm{DM}$ and $\Delta \mathrm{FD}$ parameters for this simulation are shown in magenta.

As with the previous set of simulations, all of the values shown in Figures 4.3, 4.4, 4.5, and 4.6 come from fitting for all parameters: DMX, all FD parameters, and 
a JUMP. We report $\mathcal{R}_{\text {rms }}, \chi_{\mathrm{r}}^{2}, \Delta \mathrm{DM}_{\text {rms }}, \Delta \mathrm{FD}_{\text {rms }}$, and the fit value of the JUMP for this set of fit model parameters, as well as the additional model fitting done with a single JUMP, just DMX and a single JUMP, and just all FD parameters and a single JUMP, in Tables 4.5, 4.6, 4.7, 4.8, 4.9, and 4.10. We note that for this set of simulations, when computing $\Delta \mathrm{FD}_{\mathrm{rms}}$, two different sets of FD parameters were used, one set that was fit for using DMX, FD, and a JUMP, and one where just FD and a JUMP was used, both sets come from the "Profile Evolution" simulation. This is because the FD parameters are covariant with DMX and the values change slightly depending on what parameters are fit for.

\subsection{Results}

Here we describe the results of the simulations described in $\S 4.4$ for each pulsar. Figures $4.2,4.3,4.4,4.5$, and 4.6 show the results of simulation analyses when we fit for all parameters (DMX, all FD parameters, and a JUMP). Figures 4.2 and 4.6 shows the $\triangle \mathrm{DM}$ (the difference between the injected and recovered DM, $\widehat{\mathrm{DMX}}-\mathrm{DMX}$ ) for each simulated epoch for all MSPs. Each set of simulations is split into two sets, Figure 4.2 shows the simulations described in $§ 4.4 .1$ Figure 4.6 shows the $\triangle \mathrm{DM}$ for the simulations described in $\S 4.4 .2$. In these Figures, we again note that the $\Delta \mathrm{DM}$ values have been mean subtracted as described in $\S 4.4 .1$, so we expect all points to be scattered around a mean of zero. This allows for better visualization of the spread in $\Delta \mathrm{DM}$ between different simulations, where a tighter spread indicates more precise recovery of the injected values. We also note that there 
are a few $\Delta \mathrm{DM}$ values that have particularly large error bars. This is an artifact of the $\widehat{\mathrm{DMX}}$ bin sizes. Points with these larger uncertainties only have higher frequency $1400 \mathrm{MHz}$ simulated observations within the fifteen or six day window leading to a less accurate $\widehat{\mathrm{DMX}}$.

Figures $4.3,4.4$, and 4.5 show the $\triangle \mathrm{FD}$ parameters (the difference between the injected and recovered $\mathrm{FD}$ parameters, $\widehat{\mathrm{FD}_{\mathrm{i}}}-\mathrm{FD}_{\mathrm{i}}$ ) for for all simulated MSPs, with the top and bottom sets of panels broken up by simulation. The left hand plots in these Figures show $\triangle \mathrm{FD}$ for each individual FD parameter in each MSP. The right hand plots show the total time delay described by the $\Delta \mathrm{FD}$ parameters calculated using Eq. 4.4, as a function of radio frequency. For all simulations without scatter broadening, when we fit for all parameters, the resulting $\Delta \mathrm{FD}$ parameters are distributed around zero, and within $1 \sigma$ of the injected values as shown in Figures $4.2,4.3,4.4,4.5$, and 4.6 .

We also compare how fitting for different combinations of DMX, FD parameters, and a JUMP affect both the timing residuals, quantified by $\mathcal{R}_{\text {rms }}$, and the timing model, quantified by $\chi_{\mathrm{r}}^{2}$, as reported in Tables 4.5, 4.6, 4.7, 4.8, 4.9, and 4.10. These tables also list the values of $\Delta \mathrm{DM}_{\mathrm{rms}}$ and $\Delta \mathrm{FD}_{\mathrm{rms}}$, which are used to determine how precisely $\widehat{\mathrm{DMX}}$ and $\widehat{\mathrm{FD}}$ are recovered. A large value means that the parameters are recovered less precisely, while smaller values indicate a more precise recovery.

As expected, we see that fitting for additional parameters, e.g., adding FD parameters even when none have been injected into the simulation, does not negatively impact the $\mathcal{R}_{\mathrm{rms}}$ for any simulated data sets. The $\chi_{\mathrm{r}}^{2}$ for each fit also appear 
to be generally unaffected by the addition of more model parameters, however this is due to both the slightly decreased $\chi^{2}$ value of these fits, and the reporting of $\chi_{\mathrm{r}}^{2}$ to only two decimal places. Further, when adding additional parameters to the simulations, e.g., DM variations or FD parameters, the recovered $\mathcal{R}_{\text {rms }}$ when all injected parameters are fit for agree as expected, confirming our methods.

\subsubsection{Discussion of Frequency-Independent Profile Simulations}

As FD parameters primarily model variations in the pulse profile with observing frequency (Zhu et al., 2015; Arzoumanian et al., 2016), we expect that $\widehat{\mathrm{FD}}$ should all be consistent with zero, and $\Delta \mathrm{FD}_{\text {rms }}$ should be very small for these simulations . The exception would be if the profiles are directly shifted in time, or altered in some way (e.g., scatter broadening) as a function of frequency, as denoted in Table 4.4. The injected spectral index does not alter the shape or the profiles, and should not cause additional variations in the FD parameters. This is indeed what we find, as shown by the upper right panels of Figures 4.3, 4.4, 4.5.

In the "No Variations" simulations, we find that regardless of what combination of parameters are fit for, we recover almost the same $\mathcal{R}_{\text {rms }}$. This shows that adding additional parameters does not negatively impact the precision of our pulsar timing and confirms that they are not absorbing any additional non-frequencydependent (or white) noise in the simulated data.

For the "Time-Variable Scatter Broadening w/ Constant DM", we find that

for all simulated pulsars, the $\mathcal{R}_{\mathrm{rms}}$ and $\chi_{\mathrm{r}}^{2}$ are slightly larger than for the "No 
Table 4.5 J1744-1134 No Profile Evolution Fitting Results

\begin{tabular}{|c|c|c|c|c|c|}
\hline \multicolumn{6}{|c|}{ Simulation: No Variation } \\
\hline Fit Parameters & $\begin{array}{l}\mathcal{R}_{\mathrm{rms}} \\
(\mu \mathrm{s})\end{array}$ & $\chi_{\mathrm{r}}^{2}$ & $\begin{array}{c}\Delta \mathrm{DM}_{\mathrm{rms}} \\
\left(10^{-6} \mathrm{pc} \mathrm{cm}^{-3}\right)\end{array}$ & $\begin{array}{c}\Delta \mathrm{FD}_{\mathrm{rms}} \\
(\mu \mathrm{s})\end{array}$ & $\begin{array}{c}\text { JUMP } \\
(\mu s)\end{array}$ \\
\hline Jump & 0.14 & 1.01 & - & - & $6 \pm 0$ \\
\hline DMX \& Jump & 0.14 & 1.01 & 2.4 & - & $6 \pm 0$ \\
\hline FD \& Jump & 0.14 & 1.01 & - & 0.2 & $6 \pm 0$ \\
\hline DMX \& FD \& Jump & 0.14 & 1.01 & 2.4 & 5.0 & $6 \pm 0$ \\
\hline \multicolumn{6}{|c|}{ Simulation: DM Variations } \\
\hline Jump & 0.33 & 10.87 & - & - & $7 \pm 0$ \\
\hline DMX \& Jump & 0.14 & 1.03 & 2.8 & - & $6 \pm 0$ \\
\hline FD \& Jump & 0.21 & 4.37 & - & 2.4 & $6 \pm 0$ \\
\hline DMX \& FD \& Jump & 0.14 & 1.03 & 2.8 & 14.9 & $6 \pm 0$ \\
\hline \multicolumn{6}{|c|}{ Simulation: FD Injection } \\
\hline Jump & 33.01 & 114349.42 & - & - & $197 \pm 0$ \\
\hline DMX \& Jump & 0.56 & 11.80 & 19.1 & - & $5 \pm 0$ \\
\hline FD \& Jump & 0.14 & 1.01 & - & 0.4 & $6 \pm 0$ \\
\hline DMX \& FD \& Jump & 0.14 & 1.01 & 2.7 & 5.1 & $6 \pm 0$ \\
\hline \multicolumn{6}{|c|}{ Simulation: Time Variable Scattering w/ Constant DM } \\
\hline Jump & 0.14 & 1.02 & - & - & $6 \pm 0$ \\
\hline DMX \& Jump & 0.14 & 1.02 & 34.1 & - & $6 \pm 0$ \\
\hline FD \& Jump & 0.14 & 1.02 & - & 0.7 & $6 \pm 0$ \\
\hline DMX \& FD \& Jump & 0.14 & 1.02 & 34.1 & 4.6 & $6 \pm 0$ \\
\hline \multicolumn{6}{|c|}{ Simulation: DM \& FD Variations } \\
\hline Jump & 33.27 & 116254.67 & - & - & $198 \pm 0$ \\
\hline DMX \& Jump & 0.56 & 11.88 & 17.9 & - & $5 \pm 0$ \\
\hline FD \& Jump & 0.21 & 4.34 & - & 2.3 & $6 \pm 0$ \\
\hline DMX \& FD \& Jump & 0.14 & 1.01 & 2.5 & 20.0 & $6 \pm 0$ \\
\hline
\end{tabular}

Note. - Results of fitting simulations of PSR J1744-1134 fitting for different parameters, either just a JUMP, $\triangle \mathrm{DM}$ (DMX) and a JUMP, all FD parameters and JUMP, or $\triangle \mathrm{DM}$, all FD parameters, and a JUMP. We report five quantifiers for each fit. The root mean square (RMS) of the resulting timing residuals, $\mathcal{R}_{\text {rms }}$, where values closer to zero indicate a better fit. The reduced chi-squared of the fit, $\chi_{\mathrm{r}}^{2}$, where values closer to one indicate a better fit. The RMS of the $\Delta \mathrm{DM}$ values, $\Delta \mathrm{DM}_{\mathrm{rms}}$, where smaller values indicate that the fit is more accurately recovering the injected values of $\Delta \mathrm{DM}$. The RMS of the $\Delta \mathrm{FD}$ values, $\Delta \mathrm{FD}_{\mathrm{rms}}$, where smaller values indicate that the fit is more accurately recovering the injected FD parameters. We also report the value of the JUMP that is fit in each case. 
Table 4.6 J1744-1134 Profile Evolution Fitting Results

\begin{tabular}{|c|c|c|c|c|c|}
\hline \multicolumn{6}{|c|}{ Simulation: Profile Evolution } \\
\hline Fit Parameters & $\begin{array}{l}\mathcal{R}_{\mathrm{rms}} \\
(\mu \mathrm{s})\end{array}$ & $\chi_{\mathrm{r}}^{2}$ & $\begin{array}{c}\Delta \mathrm{DM}_{\mathrm{rms}} \\
\left(10^{-6} \mathrm{pc} \mathrm{cm}^{-3}\right)\end{array}$ & $\begin{array}{c}\Delta \mathrm{FD}_{\mathrm{rms}} \\
(\mu \mathrm{s})\end{array}$ & $\begin{array}{c}\text { JUMP } \\
(\mu \mathrm{s})\end{array}$ \\
\hline Jump & 1.53 & 79.40 & - & - & $7 \pm 0$ \\
\hline DMX \& Jump & 1.19 & 24.20 & 9.6 & - & $1 \pm 0$ \\
\hline FD \& Jump & 1.18 & 21.42 & - & - & $2 \pm 0$ \\
\hline DMX \& FD \& Jump & 1.15 & 21.30 & 3.8 & - & $1 \pm 0$ \\
\hline \multicolumn{6}{|c|}{ Simulation: Profile \& DM Variations } \\
\hline Jump & 1.70 & 112.07 & - & - & $9 \pm 0$ \\
\hline DMX \& Jump & 1.19 & 24.09 & 9.7 & - & $1 \pm 0$ \\
\hline FD \& Jump & 1.18 & 22.93 & - & 2.5 & $2 \pm 0$ \\
\hline DMX \& FD \& Jump & 1.14 & 21.19 & 3.1 & 7.0 & $1 \pm 0$ \\
\hline \multicolumn{6}{|c|}{ Simulation: Constant Scatter Broadening } \\
\hline Jump & 1.70 & 111.96 & - & - & $9 \pm 0$ \\
\hline DMX \& Jump & 1.19 & 24.11 & 10.5 & - & $1 \pm 0$ \\
\hline FD \& Jump & 1.19 & 22.95 & - & 2.3 & $2 \pm 0$ \\
\hline DMX \& FD \& Jump & 1.15 & 21.23 & 3.2 & 10.8 & $1 \pm 0$ \\
\hline \multicolumn{6}{|c|}{ Simulation: Time Variable Scatter Broadening } \\
\hline Jump & 1.70 & 111.94 & - & - & $9 \pm 0$ \\
\hline DMX \& Jump & 1.19 & 23.99 & 10.1 & - & $1 \pm 0$ \\
\hline FD \& Jump & 1.18 & 22.82 & - & 2.4 & $2 \pm 0$ \\
\hline DMX \& FD \& Jump & 1.14 & 21.11 & 3.3 & 3.5 & $1 \pm 0$ \\
\hline
\end{tabular}

Note. - Same as Table 4.5 but for the simulations with pulse profile evolution with radio frequency. 
Table 4.7 B1855+09 No Profile Evolution Fitting Results

\begin{tabular}{|c|c|c|c|c|c|}
\hline \multicolumn{6}{|c|}{ Simulation: No Variation } \\
\hline Fit Parameters & $\begin{array}{c}\mathcal{R}_{\mathrm{rms}} \\
(\mu \mathrm{s})\end{array}$ & $\chi_{\mathrm{r}}^{2}$ & $\begin{array}{c}\Delta \mathrm{DM}_{\mathrm{rms}} \\
\left(10^{-6} \mathrm{pc} \mathrm{cm}^{-3}\right)\end{array}$ & $\begin{array}{l}\Delta \mathrm{FD}_{\mathrm{rms}} \\
\quad(\mu \mathrm{s})\end{array}$ & $\begin{array}{c}\text { JUMP } \\
(\mu \mathrm{s})\end{array}$ \\
\hline Jump & 0.05 & 1.49 & - & - & $120 \pm 0$ \\
\hline DMX \& Jump & 0.05 & 1.48 & 1.0 & - & $120 \pm 0$ \\
\hline FD \& Jump & 0.05 & 1.49 & - & 0.1 & $120 \pm 0$ \\
\hline DMX \& FD \& Jump & 0.05 & 1.48 & 1.0 & 2.3 & $121 \pm 0$ \\
\hline \multicolumn{6}{|c|}{ Simulation: DM Variations } \\
\hline Jump & 1.55 & 3652.14 & - & - & $83 \pm 0$ \\
\hline DMX \& Jump & 0.05 & 1.53 & 0.9 & - & $120 \pm 0$ \\
\hline FD \& Jump & 1.20 & 2872.38 & - & 18.2 & $125 \pm 0$ \\
\hline DMX \& FD \& Jump & 0.05 & 1.53 & 0.9 & 1.6 & $120 \pm 0$ \\
\hline \multicolumn{6}{|c|}{ Simulation: FD Injection } \\
\hline Jump & 7.02 & 37645.77 & - & - & $-167 \pm 0$ \\
\hline DMX \& Jump & 0.38 & 118.13 & 14.0 & - & $93 \pm 0$ \\
\hline FD \& Jump & 0.05 & 1.44 & - & 0.1 & $120 \pm 0$ \\
\hline DMX \& FD \& Jump & 0.05 & 1.43 & 1.3 & 1.2 & $121 \pm 0$ \\
\hline \multicolumn{6}{|c|}{ Simulation: Time Variable Scattering w/ Constant DM } \\
\hline Jump & 0.33 & 109.48 & - & - & $121 \pm 0$ \\
\hline DMX \& Jump & 0.13 & 15.43 & 123.4 & - & $120 \pm 0$ \\
\hline FD \& Jump & 0.33 & 108.34 & - & 1.2 & $119 \pm 0$ \\
\hline DMX \& FD \& Jump & 0.13 & 14.57 & 123.3 & 2.3 & $120 \pm 0$ \\
\hline \multicolumn{6}{|c|}{ Simulation: DM \& FD Variations } \\
\hline Jump & 8.09 & 52073.18 & - & - & $-204 \pm 0$ \\
\hline DMX \& Jump & 0.38 & 117.69 & 13.0 & - & $93 \pm 0$ \\
\hline FD \& Jump & 1.20 & 2871.78 & - & 18.2 & $125 \pm 0$ \\
\hline DMX \& FD \& Jump & 0.05 & 1.48 & 1.4 & 0.2 & $121 \pm 0$ \\
\hline
\end{tabular}

Note. - Results of fitting simulations of PSR B1855+09 fitting for different parameters, either just a JUMP, $\triangle \mathrm{DM}$ (DMX) and a JUMP, all FD parameters and JUMP, or $\triangle \mathrm{DM}$, all FD parameters, and a JUMP. We report five quantifiers for each fit. The root mean square (RMS) of the resulting timing residuals, $\mathcal{R}_{\text {rms }}$, where values closer to zero indicate a better fit. The reduced chi-squared of the fit, $\chi_{\mathrm{r}}^{2}$, where values closer to one indicate a better fit. The RMS of the $\Delta \mathrm{DM}$ values, $\Delta \mathrm{DM}_{\mathrm{rms}}$, where smaller values indicate that the fit is more accurately recovering the injected values of $\Delta \mathrm{DM}$. The RMS of the $\Delta \mathrm{FD}$ values, $\Delta \mathrm{FD}_{\mathrm{rms}}$, where smaller values indicate that the fit is more accurately recovering the injected FD parameters. We also report the value of the JUMP that is fit in each case. 
Table 4.8 B1855+09 Profile Evolution Fitting Results

\begin{tabular}{|c|c|c|c|c|c|}
\hline \multicolumn{6}{|c|}{ Simulation: Profile Evolution } \\
\hline Fit Parameters & $\begin{array}{l}\mathcal{R}_{\mathrm{rms}} \\
(\mu \mathrm{s})\end{array}$ & $\chi_{\mathrm{r}}^{2}$ & $\begin{array}{c}\Delta \mathrm{DM}_{\mathrm{rms}} \\
\left(10^{-6} \mathrm{pc} \mathrm{cm}^{-3}\right)\end{array}$ & $\begin{array}{c}\Delta \mathrm{FD}_{\mathrm{rms}} \\
(\mu \mathrm{s})\end{array}$ & $\begin{array}{c}\text { JUMP } \\
(\mu \mathrm{s})\end{array}$ \\
\hline Jump & 1.90 & 19.04 & - & - & $117 \pm 0$ \\
\hline DMX \& Jump & 1.69 & 14.28 & 2.4 & - & $88 \pm 0$ \\
\hline FD \& Jump & 1.47 & 12.31 & - & - & $35 \pm 1$ \\
\hline DMX \& FD \& Jump & 1.48 & 12.43 & 1.6 & - & $67 \pm 2$ \\
\hline \multicolumn{6}{|c|}{ Simulation: Profile \& DM Variations } \\
\hline Jum & 2.06 & 22.67 & - & - & $79 \pm 0$ \\
\hline DMX \& Jump & 1.69 & 14.25 & 2.7 & - & $88 \pm 0$ \\
\hline FD \& Jump & 1.90 & 20.36 & - & 18.2 & $40 \pm 1$ \\
\hline DMX \& FD \& Jump & 1.48 & 12.41 & 1.7 & 2.0 & $67 \pm 2$ \\
\hline \multicolumn{6}{|c|}{ Simulation: Constant Scatter Broadening } \\
\hline Jump & 2.08 & 22.62 & - & - & $80 \pm 0$ \\
\hline DMX \& Jump & 1.70 & 14.25 & 3.2 & - & $89 \pm 0$ \\
\hline FD \& Jump & 1.90 & 20.10 & - & 16.8 & $38 \pm 1$ \\
\hline DMX \& FD \& Jump & 1.47 & 12.17 & 1.6 & 4.1 & $66 \pm 2$ \\
\hline \multicolumn{6}{|c|}{ Simulation: Time Variable Scatter Broadening } \\
\hline Jump & 2.05 & 22.15 & - & - & $80 \pm 0$ \\
\hline DMX \& Jump & 1.71 & 14.34 & 26.0 & - & $89 \pm 0$ \\
\hline FD \& Jump & 1.87 & 19.65 & - & 16.9 & $38 \pm 1$ \\
\hline DMX \& FD \& Jump & 1.48 & 12.28 & 25.9 & 3.7 & $66 \pm 2$ \\
\hline
\end{tabular}

Note. - Same as Table 4.7 but for the simulations with pulse profile evolution with radio frequency. 
Table 4.9 B1953+29 No Profile Evolution Fitting Results

\begin{tabular}{|c|c|c|c|c|c|}
\hline \multicolumn{6}{|c|}{ Simulation: No Variation } \\
\hline Fit Parameters & $\begin{array}{l}\mathcal{R}_{\mathrm{rms}} \\
(\mu \mathrm{s})\end{array}$ & $\chi_{\mathrm{r}}^{2}$ & $\begin{array}{c}\Delta \mathrm{DM}_{\mathrm{rms}} \\
\left(10^{-6} \mathrm{pc} \mathrm{cm}^{-3}\right)\end{array}$ & $\begin{array}{c}\Delta \mathrm{FD}_{\mathrm{rms}} \\
(\mu \mathrm{s})\end{array}$ & $\begin{array}{c}\text { JUMP } \\
(\mu \mathrm{s})\end{array}$ \\
\hline Jump & 0.18 & 1.14 & - & - & $-455 \pm 0$ \\
\hline DMX \& Jump & 0.18 & 1.13 & 3.7 & - & $-455 \pm 0$ \\
\hline FD \& Jump & 0.18 & 1.14 & - & 0.1 & $-455 \pm 0$ \\
\hline DMX \& FD \& Jump & 0.18 & 1.13 & 3.7 & 0.2 & $-455 \pm 0$ \\
\hline \multicolumn{6}{|c|}{ Simulation: DM Variations } \\
\hline Jump & 4.20 & 9668.28 & - & - & $-411 \pm 0$ \\
\hline DMX \& Jump & 0.18 & 1.21 & 6.0 & - & $-455 \pm 0$ \\
\hline FD \& Jump & 4.05 & 9493.81 & - & 35.5 & $-472 \pm 0$ \\
\hline DMX \& FD \& Jump & 0.18 & 1.21 & 6.1 & 0.1 & $-455 \pm 0$ \\
\hline \multicolumn{6}{|c|}{ Simulation: FD Injection } \\
\hline Jump & 11.08 & 4592.29 & - & - & $-650 \pm 0$ \\
\hline DMX \& Jump & 6.82 & 1734.07 & 348.3 & - & $-477 \pm 0$ \\
\hline FD \& Jump & 0.18 & 1.20 & - & 0.1 & $-455 \pm 0$ \\
\hline DMX \& FD \& Jump & 0.18 & 1.19 & 4.8 & 0.2 & $-455 \pm 0$ \\
\hline \multicolumn{6}{|c|}{ Simulation: Time Variable Scattering w/ Constant DM } \\
\hline Jump & 1.08 & 614.52 & - & - & $-443 \pm 0$ \\
\hline DMX \& Jump & 0.56 & 12.98 & 432.4 & - & $-463 \pm 0$ \\
\hline FD \& Jump & 1.08 & 580.55 & - & 18.0 & $-472 \pm 0$ \\
\hline DMX \& FD \& Jump & 0.27 & 3.33 & 433.3 & 11.5 & $-460 \pm 0$ \\
\hline \multicolumn{6}{|c|}{ Simulation: DM \& FD Variations } \\
\hline Jump & 10.78 & 12816.39 & - & - & $-606 \pm 0$ \\
\hline DMX \& Jump & 6.83 & 1739.88 & 343.5 & - & $-476 \pm 0$ \\
\hline FD \& Jump & 4.05 & 9491.51 & - & 35.5 & $-472 \pm 0$ \\
\hline DMX \& FD \& Jump & 0.19 & 1.23 & 5.9 & 0.1 & $-455 \pm 0$ \\
\hline
\end{tabular}

Note. - Results of fitting simulations of PSR B1953+29 fitting for different parameters, either just a JUMP, $\triangle \mathrm{DM}(\mathrm{DMX})$ and a JUMP, all FD parameters and JUMP, or $\triangle \mathrm{DM}$, all FD parameters, and a JUMP. We report five quantifiers for each fit. The root mean square (RMS) of the resulting timing residuals, $\mathcal{R}_{\text {rms }}$, where values closer to zero indicate a better fit. The reduced chi-squared of the fit, $\chi_{\mathrm{r}}^{2}$, where values closer to one indicate a better fit. The RMS of the $\Delta \mathrm{DM}$ values, $\Delta \mathrm{DM}_{\mathrm{rms}}$, where smaller values indicate that the fit is more accurately recovering the injected values of $\Delta \mathrm{DM}$. The RMS of the $\Delta \mathrm{FD}$ values, $\Delta F D_{\text {rms }}$, where smaller values indicate that the fit is more accurately recovering the injected FD parameters. We also report the value of the JUMP that is fit in each case. 
Table 4.10 B1953+29 No Profile Evolution Fitting Results

\begin{tabular}{|c|c|c|c|c|c|}
\hline \multicolumn{6}{|c|}{ Simulation: Profile Evolution } \\
\hline Fit Parameters & $\begin{array}{l}\mathcal{R}_{\mathrm{rms}} \\
(\mu \mathrm{s})\end{array}$ & $\chi_{\mathrm{r}}^{2}$ & $\begin{array}{c}\Delta \mathrm{DM}_{\mathrm{rms}} \\
\left(10^{-6} \mathrm{pc} \mathrm{cm}^{-3}\right)\end{array}$ & $\begin{array}{c}\Delta \mathrm{FD}_{\mathrm{rms}} \\
(\mu \mathrm{s})\end{array}$ & $\begin{array}{c}\text { JUMP } \\
(\mu \mathrm{s})\end{array}$ \\
\hline Jump & 44.50 & 4657.17 & - & - & $-443 \pm 0$ \\
\hline DMX \& Jump & 6.22 & 127.85 & 208.6 & - & $1163 \pm 0$ \\
\hline FD \& Jump & 7.28 & 136.28 & - & - & $1205 \pm 1$ \\
\hline DMX \& FD \& Jump & 2.30 & 20.27 & 7.6 & - & $1007 \pm 1$ \\
\hline \multicolumn{6}{|c|}{ Simulation: Profile \& DM Variations } \\
\hline Jump & 43.55 & 4514.72 & - & - & $-398 \pm 0$ \\
\hline DMX \& Jump & 6.23 & 128.29 & 208.3 & - & $1163 \pm 0$ \\
\hline FD \& Jump & 8.09 & 228.31 & - & 29.8 & $1197 \pm 1$ \\
\hline DMX \& FD \& Jump & 2.30 & 20.26 & 6.6 & 0.9 & $1007 \pm 1$ \\
\hline \multicolumn{6}{|c|}{ Simulation: Constant Scatter Broadening } \\
\hline Jump & 43.43 & 4438.66 & - & - & $-386 \pm 0$ \\
\hline DMX \& Jump & 6.47 & 137.58 & 213.4 & - & $1166 \pm 0$ \\
\hline FD \& Jump & 7.88 & 217.56 & - & 47.5 & $1179 \pm 1$ \\
\hline DMX \& FD \& Jump & 2.30 & 20.02 & 7.2 & 11.1 & $1000 \pm 1$ \\
\hline \multicolumn{6}{|c|}{ Simulation: Time Variable Scatter Broadening } \\
\hline Jump & 43.46 & 4450.10 & - & - & $-386 \pm 0$ \\
\hline DMX \& Jump & 6.47 & 137.85 & 234.3 & - & $1167 \pm 0$ \\
\hline FD \& Jump & 8.02 & 231.50 & - & 48.0 & $1179 \pm 1$ \\
\hline DMX \& FD \& Jump & 2.31 & 20.09 & 99.2 & 11.5 & $1001 \pm 1$ \\
\hline
\end{tabular}

Note. - Same as Table 4.9 but for the simulations with pulse profile evolution with radio frequency. 
Variation" simulation. The effect of the scattering delays are obvious from the light green points in Figure 4.2, where the larger the average value, and hence spread of, $\tau_{\mathrm{d}}$, the less accurate and more variable the resulting $\Delta \mathrm{DM}$, and subsequently $\widehat{\mathrm{DMX}}$, is. This is less obvious in Figure 4.3, but in Figures 4.4 and 4.5, the inability to recover accurate FD parameters due to larger average injected $\tau_{\mathrm{d}}$ values is apparent as the light green curve is not consistent with zero. This indicates that the DMX and FD parameters cannot appropriately account for time-variable scattering delays, though the larger variation in the $\triangle \mathrm{DM}$ values suggest that the additional delays from scattering are absorbed by $\widehat{\mathrm{DMX}}$, showing a clear covariance between these two frequency-dependent effects.

For all other simulations in this set, the resulting $\mathcal{R}_{\mathrm{rms}}$ and $\chi_{\mathrm{r}}^{2}$ show that when the appropriate parameters are fit for, all frequency-dependent delays are accounted for, affirming our expectations. When only FD parameters are injected and all parameters are fit for, $\Delta \mathrm{FD}_{\mathrm{rms}}$ increases and $\Delta \mathrm{DM}_{\mathrm{rms}}$ either remains constant or decreases. This is indicative of a small covariance between DMX and the FD parameters, and shows that FD parameters are more susceptible to variations than DMX is when additional frequency-dependent effects are present and fit. While this is expected (Zhu et al., 2015), it increases our confidence that when there is very little or no scattering, the FD parameters are absorbing very little of the dispersive delays. In these cases, such as PSR J1744-1134, we can be reasonably confident that the injected DM is being recovered.

In real pulsar timing data, both DM variations and additional non- $\nu^{-2}$ frequencydependent effects are present. The results of this set of simulations shows that we 
can accurately recover the full injected delay, when the scattering timescale is very small or zero, giving us confidence in both our methods, and our ability to use this set of simulations as a comparison to our more complex simulations.

\subsubsection{Discussion of Frequency-Dependent Profile Simulations}

In the "Profile Evolution" simulations, $\chi_{\mathrm{r}}^{2}$ is consistent when fitting for just FD parameters and all parameters, for PSRs J1744-1134 and B1855+09. However, for PSR B1953+29, $\chi_{\mathrm{r}}^{2}$ is lower when fitting for all parameters compared to just FD parameters. As PSR B1953+29 has a much higher DM, this suggests that $\widehat{\mathrm{DMX}}$ may absorb more of the delays from profile evolution at higher DMs. It is possible that this is because at higher DMs, the profile evolution may be primarily dominated by scattering. Since scattering scales in a similarly frequency-dependent way to DM, $\widehat{\mathrm{DMX}}$ may absorb the effects of scatter broadening more at higher DMs. We note, however, that as we have simulated only three MSPs it is difficult to verify this. To fully explore this relationship would require additional simulations of comparable length exploring not only the scale of DM, but also the size of the DM variations and the number of FD parameters, and as such is beyond the scope of this work. Additionally, since we recover very similar values of $\mathcal{R}_{\text {rms }}$ and $\chi_{\mathrm{r}}^{2}$ for all MSPs using both methods of fitting, $\widehat{\mathrm{DMX}}$ likely fits out very little of this intrinsic profile evolution, which is expected (Zhu et al., 2015; Arzoumanian et al., 2016).

In all other simulations in this set, we find that the best values of $\mathcal{R}_{\text {rms }}$ and $\chi_{\mathrm{r}}^{2}$ occur when we fit for all parameters, which is consistent with the previous sim- 
ulations discussed in §4.5.1. For the "Time-Variable Scatter Broadening" simulation, we note that $\mathcal{R}_{\text {rms }}$ is comparable to those obtained in the other simulations in this set, despite Figure 4.6 showing that when the average $\tau_{\mathrm{d}}$ is large, $\widehat{\mathrm{DMX}}$ is much less accurate and more variable. This is consistent with the results from the "Time-Variable Scatter Broadening w/ Constant DM " and suggests that the average scatter broadening is completely fit out by the FD parameters, while the time variations in the injected $\tau_{\mathrm{d}}$ are primarily absorbed by the DMX parameters. This again shows the clear covariance between DM and scattering.

For PSR J1744-1134, which has both the lowest DM and the most FD parameters of our simulated pulsars, we find that $\Delta \mathrm{FD}_{\mathrm{rms}}$ decreases while $\Delta \mathrm{DM}_{\mathrm{rms}}$ stays comparable when going from a constant to time-varying injected $\tau_{\mathrm{d}}$. This is in contrast to both PSRs B1855+09 and B1953+29, which show an increase in $\Delta \mathrm{DM}_{\mathrm{rms}}$ but a roughly constant $\Delta \mathrm{FD}_{\mathrm{rms}}$ when $\tau_{\mathrm{d}}$ varies with time. It is difficult to determine if this suggests that pulsars with more FD parameters and/or smaller $\tau_{\mathrm{d}}$ are less effected by time-varying $\tau_{\mathrm{d}}$, or if this is an artifact of pulsars we have chosen to simulate. In all simulations in this set for PSR J1744-1134 $\Delta F D_{\text {rms }}$ is larger when fitting for all parameters than just FD parameters, which may similarly suggest that the covariance between DMX and the FD parameters is larger for more FD parameters and/or smaller DM or DM variations. In either case, a comprehensive analysis of this potential relationship would involve exploring a large parameter space, mentioned above, that is beyond the scope of this work.

The difference between the constant and time-varying scatter broadening simulations most clearly shows that while there may be a covariance between DMX and 
FD parameters, it is very small. As the FD parameters are fit over the full data set, the differences in $\Delta \mathrm{FD}_{\mathrm{rms}}$ from a constant to time-varying $\tau_{\mathrm{d}}$ are much smaller than those of $\Delta \mathrm{DM}_{\mathrm{rms}}$. Since the DMX are fit as a piece-wise function over small timescales, they account for most of the additional time-varying scattering delays. While this means $\widehat{\mathrm{DMX}}$ may not be as accurate, we can see that it does not seem to have a large effect on $\mathcal{R}_{\text {rms }}$, since for both scattering simulations in this set this value is comparable to the smallest $\mathcal{R}_{\text {rms }}$ in the baseline "Profile Evolution" simulation.

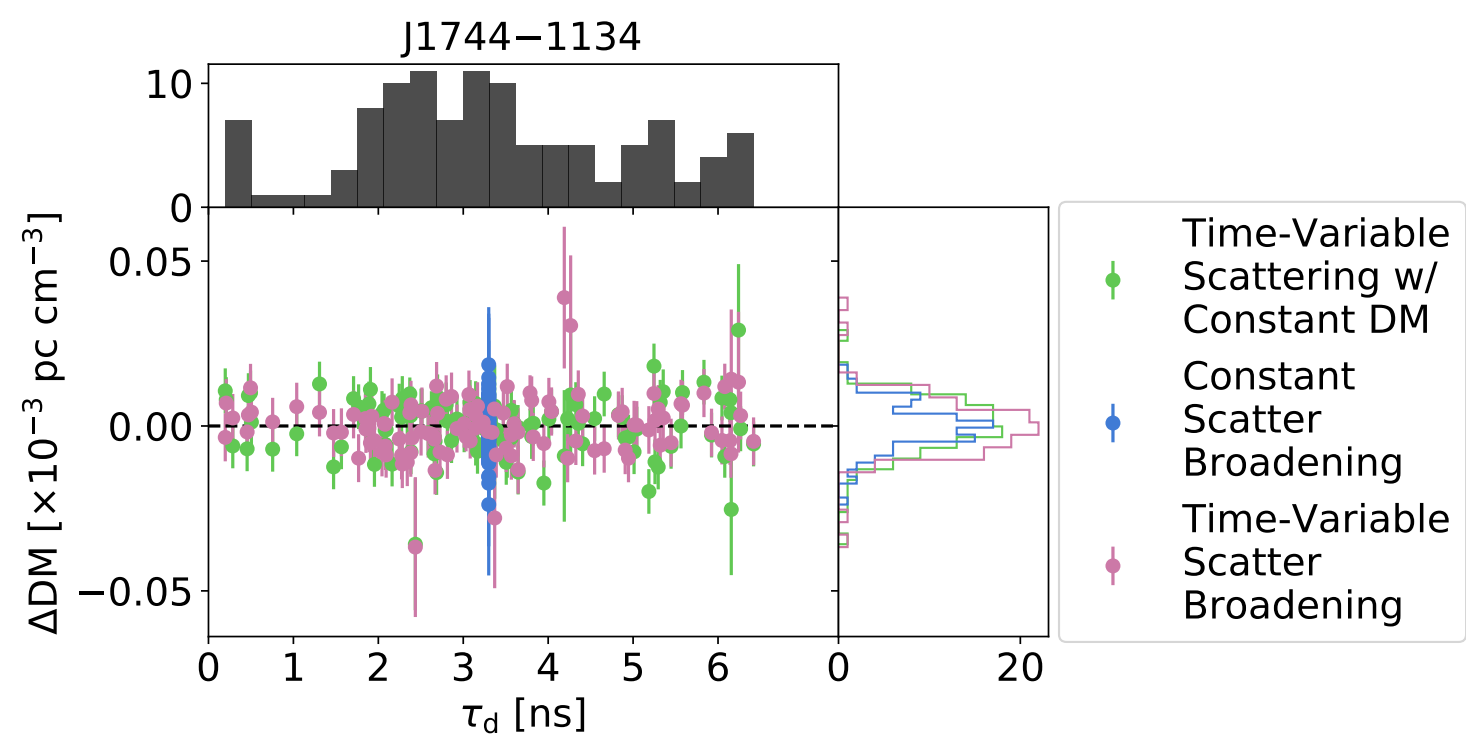

Figure $4.7 \Delta \mathrm{DM}$ value $(\widehat{\mathrm{DMX}}-\mathrm{DMX})$ versus the injected scattering timescale $(\mathrm{s}) \tau_{\mathrm{d}}$ within each DMX bin for PSR J1744-1134. There are the same number of points for each simulation shown. We can see that in the case of time-variable scattering delays, the injected DMX value is recovered less accurately shown by the larger spread in the green and magenta $\Delta \mathrm{DM}$ points compared to the blue points. The top histogram shows the distribution of injected $\tau_{\mathrm{d}}$ while the right histogram shows the distribution of $\triangle \mathrm{DM}$. We clearly see no correlation between the injected value $\tau_{\mathrm{d}}$ and the $\Delta \mathrm{DM}$, showing that time-variable scattering only serves to make $\widehat{\mathrm{DMX}}$ more variable and less accurate, though minimally for small average values of $\tau_{\mathrm{d}}$. 


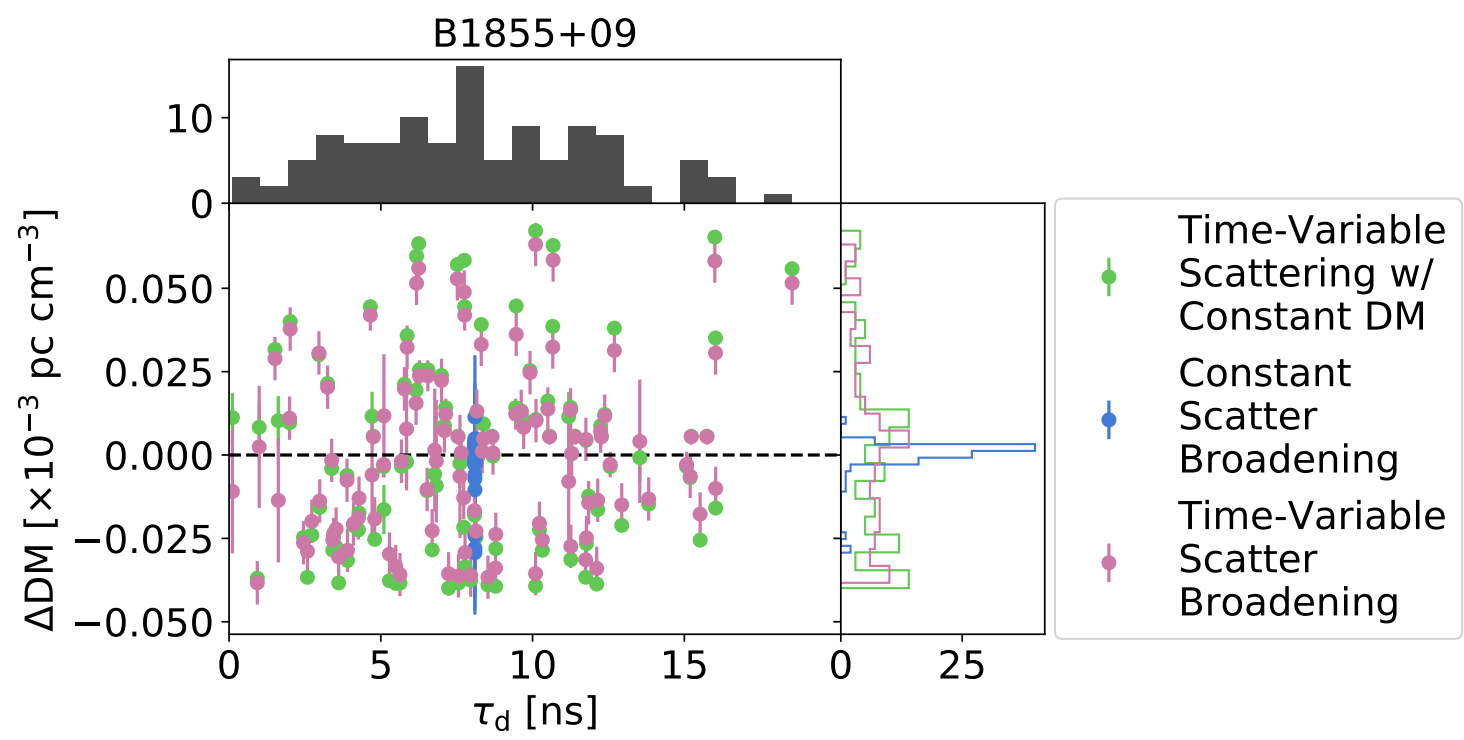

Figure 4.8 $\triangle \mathrm{DM}$ value $(\widehat{\mathrm{DMX}}-\mathrm{DMX})$ versus the injected scattering timescale(s) $\tau_{\mathrm{d}}$ within each DMX bin for PSR B1855+09. There are the same number of points for each simulation shown. We can see that in the case of time-variable scattering delays, the injected DMX value is recovered less accurately, as shown by the larger spread in the green and magenta $\Delta \mathrm{DM}$ points compared to the blue points. The top histogram shows the distribution of injected $\tau_{\mathrm{d}}$ while the right histogram shows the distribution of $\triangle \mathrm{DM}$. We clearly see no correlation between the injected value $\tau_{\mathrm{d}}$ and the $\Delta \mathrm{DM}$, showing that time-variable scattering only serves to make $\widehat{\mathrm{DMX}}$ more variable and less accurate.

\subsection{Implications for Precision Pulsar Timing}

The results of our simulations and analyses are important when considering the use of PTAs to detect gravitational waves (e.g., McLaughlin, 2013; Kramer \& Champion, 2013; Hobbs, 2013). In all simulated MSPs, we find that when profile evolution is present through direct injection or the use of frequency-dependent profiles, $\Delta \mathrm{FD}_{\mathrm{rms}}$ can change by an order of magnitude when all parameters are fit for, compared to just FD parameters. In general this change seems to be an increase for pulsars with more FD parameters and smaller DMs, and a decrease for pulsars with larger DMs and fewer FD parameters, however it is important to note that we 


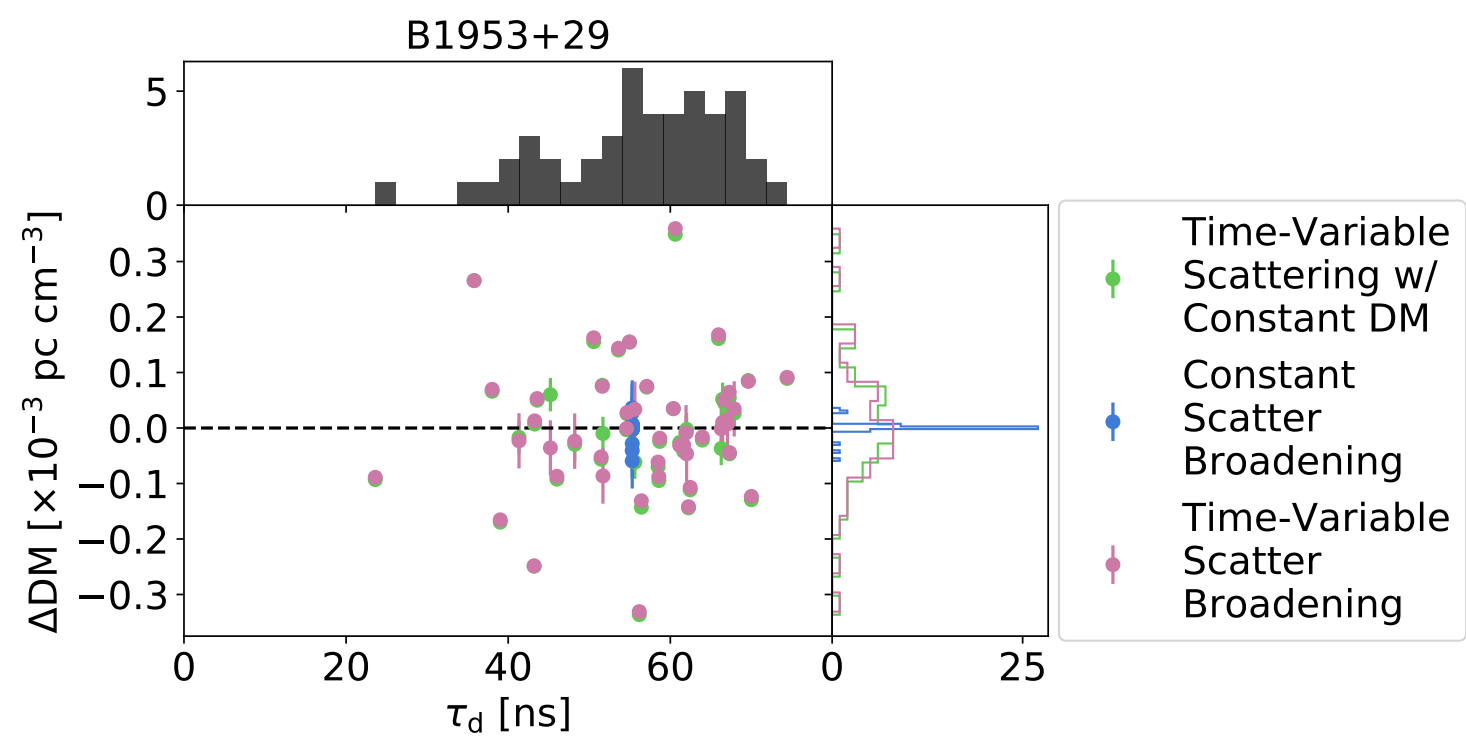

Figure $4.9 \Delta \mathrm{DM}$ value $(\widehat{\mathrm{DMX}}-\mathrm{DMX})$ versus the injected scattering timescale(s) $\tau_{\mathrm{d}}$ within each DMX bin for PSR B1953+29. There are the same number of points for each simulation shown. We can see that in the case of time-variable scattering delays, the injected DMX value is recovered less accurately shown by the larger spread in the green and magenta $\Delta \mathrm{DM}$ points compared to the blue points. The top histogram shows the distribution of injected $\tau_{\mathrm{d}}$ while the right histogram shows the distribution of $\Delta \mathrm{DM}$. We clearly see no correlation between the injected value $\tau_{\mathrm{d}}$ and the $\Delta \mathrm{DM}$, showing that time-variable scattering only serves to make $\widehat{\mathrm{DMX}}$ more variable and less accurate.

have only simulated three pulsars in this work. Regardless, this is evidence of the covariance between DMX and FD parameters especially in the simulations where no scattering delays are injected, however the effect on $\mathcal{R}_{\text {rms }}$ in negligible, as it is always at a minimum when all appropriate parameters have been fit for. Additionally, $\chi_{\mathrm{r}}^{2}$ is almost always closest to one when fitting for all parameters, showing that the addition of FD parameters does not make the timing model fit worse.

Additionally, one can see from Figures 4.2 and 4.6 that, when no time-variable scattering delays are injected, as long as the DMX fit spans both frequency bands, we can recover the injected DMX, regardless of the DM variations, the nominal DM 
value, or the number of FD parameters. This shows that, as expected (Zhu et al., 2015; Arzoumanian et al., 2016), our models and fitting are able to, in principle, separate out the physical variations in DM from any effects modeled out by FD parameters. We can conclude that though there is a definitive covariance between DMX and the FD parameters, it is very small, and does not affect the precision of the pulsar timing.

The most interesting result and impact found in our simulations is when timevariable $\tau_{\mathrm{d}}$ are injected. It is apparent from Figures 4.2, 4.3, 4.4, 4.5, and 4.6 that these time-variable delays decrease the accuracy and increase the variability of $\widehat{\mathrm{DMX}}$ as well as $\widehat{\mathrm{FD}}$, and that the level of inaccuracy and variability increases with larger values of $\tau_{\mathrm{d}}$. This seems to indicate that the time delays due to scattering are primarily absorbed by the DMX values indicating a clear covariance between the two frequency-dependent effects. This shows that in real pulsar data, larger scattering timescales will result in some of the variations in $\widehat{\mathrm{DMX}}$. However, in our most realistic simulation, "Time-Variable Scatter Broadening", $\mathcal{R}_{\text {rms }}$ is within $10 \mathrm{~ns}$ of the minimum expected $\mathcal{R}_{\text {rms }}$ given in our baseline "Profile Evolution" simulation. So even though the time-variable scattering clearly changes the $\widehat{\mathrm{DMX}}$ and $\widehat{\mathrm{FD}}$, it has a minimal effect on $\mathcal{R}_{\text {rms }}$, at least for the three pulsars simulated in this work.

Since $\widehat{\mathrm{DMX}}$ have a much larger spread with varying $\tau_{\mathrm{d}}$, we also look to see if there is a correlation between the two parameters. The $\Delta \mathrm{DM}$ values are plotted against the values of $\tau_{\mathrm{d}}$ injected within each DMX epoch in Figures 4.7, 4.8, and 4.9. In all cases, we can see that for simulations with time-variable scattering delays (green and magenta), the larger the average value, and hence spread of, $\tau_{\mathrm{d}}$, the larger 
the spread in $\Delta \mathrm{DM}$. For simulations with constant scattering delays (blue), there is very little variability in $\Delta \mathrm{DM}$. The $\Delta \mathrm{DM}_{\mathrm{rms}}$ seems to be larger when there are no DM variations or frequency-dependent profiles injected, though the spread of the resulting $\Delta \mathrm{DM}$ values are roughly the same magnitude between the two simulations. Most importantly though, there appears to be no correlation between the injected $\tau_{\mathrm{d}}$ and $\widehat{\mathrm{DMX}}$. This is likely because the FD parameters fit out the mean injected $\tau_{\mathrm{d}}$, so when the distribution of injected $\tau_{\mathrm{d}}$ is more spread out (see top histograms), $\widehat{\mathrm{DMX}}$ must absorb a larger portion of the scattering delays, resulting in a larger spread in $\Delta \mathrm{DM}$.

The fact that $\mathcal{R}_{\text {rms }}$ appears to be relatively unaffected by time-variable scattering delays shows that the delays from frequency-dependent effects are modeled out due to the covariance between DM and scattering. As GWs are not radio-frequency dependent, this covariance therefore does not preclude current PTAs from potential detection, though it could induce additional red noise processes in the data.

However, accurate measurements of DM are extremely important for precision pulsar timing and for understanding noise in the timing data. In particular, for experiments designed to detect nanohertz GWs, advanced noise modeling techniques such as those discussed in Arzoumanian et al. (2020b) will benefit greatly from disentangling the covariances between DM and scattering where precision down to $\sim$ 100s of nanoseconds is required (e.g., Lam et al., 2018b; Lam, 2018; Lam et al., 2019). Techniques such as cyclic spectroscopy (Dolch et al., 2020; Turner et al., 2020), or alternative methods of quantifying time-variable scattering such as those described in Main et al. (2020) will be necessary to break this covariance. 


\subsection{Conclusions}

Here for the first time we have used the PSRSIGSIM to simulate pulsar data for three different MSPs with DM variations, pulse profile evolution with frequency, and time-variable scatter broadening to explore the covariances between these effects. We show that the PSRSIGSIM is able to efficiently simulate large amounts of unevenly sampled data spanning long timescales which can then be processed using other standard pulsar software such as PSRCHIVE and PINT to get pulse TOAs as well as fit timing models. The different delays that were injected into the simulated data such as DMX, and direct shifts corresponding to the FD parameters can be accurately recovered using these softwares. This emphasizes not only the usefulness of the PSRSIGSIM, but also that the standard timing model fitting procedures, such as those implemented in PINT, are able to differentiate between these different frequencydependent effects.

As an interesting first use case of the PSRSIGSIM, we explored the covariance between the DMX and FD parameters and what, if any, effect this will have on precision pulsar timing. We find that there is a definite covariance between the two as evidenced by the varying values of $\Delta F D_{\text {rms }}$ when fitting for all parameters. However, in almost all cases, when fitting for all parameters, $\mathcal{R}_{\text {rms }}$ was equivalent to the minimum expected values. This, combined with the fact that the injected values of DM and FD parameters were also recovered, shows that this covariance is small, and has a negligible effect on the precision of the pulsar timing. While this is expected, these simulations show that this covariance should have little to no 
impact on the pulsar timing.

Our simulations also find that when scatter broadening is added, the FD parameters are able to fit out the average injected $\tau_{\mathrm{d}}$. However, when time-variable scattering delays are injected, both the recovered DM and FD parameters, $\widehat{\mathrm{DMX}}$ and $\widehat{\mathrm{FD}}$, are significantly less accurate, increasing with the average injected $\tau_{\mathrm{d}}$. We find that most of this additional scattering delay is likely being absorbed by the DMX parameters, showing the covariance between these effects. While this does not seem to have a significant impact on $\mathcal{R}_{\text {rms }}$, it does imply that some of the variations in DM seen are due to variable values of $\tau_{\mathrm{d}}$, and that additional analysis and new techniques, such as cyclic spectroscopy (Dolch et al., 2020), will be needed to separate out these two effects.

These simulations represent just the beginnings of what can be done with the PSRSIGSIM. Further studies with the PSRSIGSIM may look at profile evolution in the era of wide-band pulsar timing (Pennucci et al., 2014; Alam et al., 2020c), or explore other effects not yet incorporated, such as scintillation. Further improvements to the PSRSIGSIM to create realistic data sets containing GW signals will be critical for confirming a future detection by PTAs. Additionally, the PSRsigsim offers ways to explore how future telescope upgrades, such as the ultra-wideband receiver to be installed at the GBT, will affect our pulsar timing (Skipper et al., 2019). The ability to simulate realistic pulsar data in formats commonly used allow for the potential to test different timing or searching algorithms, explore the effects of different parameters, and test the recovery of input signals, such as GWs, in a new, meaningful way. 


\section{Chapter 5}

\section{Conclusion}

We have studied the properties of different kinds of pulsars spanning singlepulse analyses of long-period rotating radio transients (RRATs) to the most precisely timed millisecond pulsars (MSPs), with both real and simulated data. These studies allowed us to explore the sporadic emission mechanisms of RRATs, and helped to characterize the interstellar medium (ISM) along the line-of-sight (LOS) to seven MSPs. From this characterization we were able to quantify the different white noise contributions, particularly those due to diffractive interstellar scattering (DISS), within these observations which will aid in the detection of nanohertz gravitational waves (GWs) by the North American Nanohertz Observatory for Gravitational Waves (NANOGrav) pulsar timing array (PTA). Further, we constructed the Pulsar Signal Simulator (PSRSigsim) Python package and were able to show not only its usefulness in simulating pulsar observations, but also in probing the covariances of radio frequency-dependent pulsar timing model parameters and determining what the implications of these covariances are for GW detection.

\subsection{Understanding the Properties of RRATs}

Using 11 years of observations of the three RRATS - PSRs J1819-1458, $\mathrm{J} 1317-5759$, and $\mathrm{J} 1913+1330$ - we were able to to extract a large sample of single 
pulses, allowing us to explore various properties of these mysterious objects. For the first time we measured the spectral indices of the single pulses of these RRATs and found that they were Gaussian-distributed around a value of -0.9. Compared to the mean spectral index of -1.6 that has been found for canonical pulsars (Jankowski et al., 2018), the spectral indices for these three RRATs are flatter than may be been expected. We then explored the emission mechanisms of these three RRATs by looking at their single-pulse flux distributions. We found that two of the RRATs, PSRs J1317-5759 and J1913+1330, follow standard log-normal flux distributions, and PSR J1819-1458 showed that a power-law to the log-normal distribution was not statistically significant, suggesting that the bright pulse emission is unlikely to be from giant pulses. However, a larger number of pulses would allow for further study and testing.

Further, due to the large number of observations and single pulses, we were able to study the wait-time distributions of all three of these RRATs. While we found that all of the wait-time distributions were consistent with random pulse emission, for PSRs J1819-1458 and J1317-5759 there was a small increase in the number of pulses around wait times of $\sim 25$ periods, which we could not explain with any standard or expected emission distribution or model. Finally, we compared the flux of each single pulse with its wait-time, but found there was no correlation between the two. This eliminates emission mechanisms such as energy being stored up within the pulsars magnetic field and then released, since for this type of model we would expect the flux to increase with wait-time. While our study sheds some light on the properties of RRATs, there is still much to be learned from these enigmatic pulsars, 
which may be gleaned through large samples and studies of their single pulses.

\subsection{Importance of Quantifying Noise in PTAs and Characterizing the ISM}

For the detection of nanohertz GWs, quantifying and understanding all of the noise within a PTA is critical. A large source of this noise comes from the ISM, and as each MSP in the PTA lies at a different distance along different LOSs, each one must be characterized. In order to do this, we analyzed multi-hour continuous observations of seven MSPs currently in the NANOGrav PTA to quantify the noise contributions in each pulsar. The nature of these observations allowed us to obtain precise measurements of the scintillation parameters for all pulsars, including many of their timescales for the first time. This in turn allowed us to show that the noise contributed to all of these pulsars due to pulse scattering was quite small, $\lesssim$ 50 ns. Quantifying these scintillation parameters also showed us that for two MSPs, PSRs J0023+0923 and J1614-2230, the assumption that the ISM is a uniform medium with a scattering screen halfway between us and the pulsar cannot explain the observed scintillation parameters. Future observations utilizing NANOGrav observations will be critical to exploring and modeling the LOSs to these MSPs. This will allow us to better characterize not only the ISM but also may allow for more informed individual pulsar noise models in the future (Simon et al. in prep).

Using our measurements of the pulse time of arrival (TOA) errors due to scattering, we were also able to estimate the error expected due to pulse jitter. 
However, we found that while our scattering error measurements were very precise, our measurements of pulse jitter were limited by the signal-to-noise $(\mathrm{S} / \mathrm{N})$ of the observations. A likely reason for the $\mathrm{S} / \mathrm{N}$ limitations compared to previous studies such as Lam et al. (2019) is that we analyze just a single epoch, limiting the potential of refractive scattering to increase the $\mathrm{S} / \mathrm{N}$ of our observations (Stinebring et al., 2000). The length of these observations also allowed us to study potential variations in the pulsar dispersion measure $(\mathrm{DM})$ on $\sim$ hour long timescales. We do not expect there to be measurable variations in the DM on these timescales, and our analysis of these MSPs confirmed this to be true. Finally, we also showed that splitting up the observation into non-contiguous chunks for the timing analysis does not affect the root mean square of the pulsar timing residuals. While it was not expected to, this scenario shows that shorter but more regular observations, compared to the current $\sim 20$ minute, monthly cadence used by NANOGrav (Alam et al., 2021), such as those being done by the Canadian Hydrogen Intensity Mapping Experiment (CHIME), will be extremely useful to add to PTA data sets.

In total, our analysis shows the importance of long observations such as these in characterizing the ISM, which may allow for better constraints on the contribution of DISS noise to a pulsar's timing residuals. Further, the information observations like these yield about the ISM may help inform future, more advanced noise modeling techniques which may aid in the detection of sources of continuous GW emission (Simon et al. in prep). 


\subsection{Utilizing Simulations for Evaluating PTA Noise Budgets}

Analyzing real pulsar data and using it for quantifying the noise budget of PTAs is critical for the detection of nanohertz GWs. However, many parameters that go into a pulsar's timing model are dependent on the observing radio frequency which causes a covariance between the parameters that is difficult, if not impossible, to disentangle in real observations. In part to look into the effects of these covariances, we developed the PSRSIGSIM, which can realistically simulate many of these different effects. By simulating many different realistic data sets of three MSPs in the NANOGrav PTA that had combinations of DM variations, pulse profile evolution with radio frequency, and pulse scattering broadening, we found that the largest covariance occurs when time-variable pulse scatter broadening is present. This covariance is particularly noticeable in the difference between the recovered and injected DM value.

As discussed in $\S 4$, we find a clear correlation between the mean injected scattering timescale and the spread in the recovered DM value, with larger scattering timescales corresponding to larger spreads. However, while we expect that larger mean scattering timescales will correspond to larger mean DMs, we find no correlation between the injected scattering timescale and the recovered DM value at any given epoch. This is likely because the FD parameters fit out the mean injected $\tau_{\mathrm{d}}$,

so if the spread in injected $\tau_{\mathrm{d}}$ is large, then the recovered DM will absorb more of this delay. Despite the larger spread in recovered DM when time variable scattering is present, the root mean square (RMS) of the timing residuals does not appear to 
be affected. This covariance is important to understand as it may have implications for our understanding of how much scattering, DM variations, or other frequencydependent (chromatic) effects contribute to the overall PTA noise budget. However, as this covariance does not affect the RMS of the timing residuals and these effects are chromatic, it should not prevent or delay NANOGrav, or any other PTA, from detecting the nanohertz stochastic GW background (SGWB), which is achromatic.

\subsection{Looking Ahead}

Recent results in Arzoumanian et al. (2020b) and simulations in Pol et al. (2020) suggest that PTAs will detect the nanohertz SGWB in the next couple of years. This detection will bring us into a new regime of GW astrophysics, and we will soon find ourselves not only continuing to better characterize this GW background, but also searching for, and detecting, individual sources of continuous GW emission (CWs). As PTAs get more and more sensitive and detection of these GW sources becomes a reality, fully understanding the PTA noise budget and quantifying all of its components will become increasingly important. Further observations and studies of ISM effects and covariances therein will be required to verify any GW signal, and complex, individual MSP noise models may be required for this in some cases, as described above. When possible, single-pulse studies of bright MSPs will also help to constrain the effects of pulse jitter and subsequent TOA errors in PTA data as well.

Further, continued development of software like the PSRSIGSIM will be critical 
for producing full, realistic PTA data sets that include GW injections to verify data analysis pipelines. These simulations will be critical not only for detection of the SGWB, but especially for CW sources where the signals may be much weaker. Not only with the PSRSIGSIM be useful for PTA science, but also for studying and simulating single-pulse studies for bright pulsars or RRATs. As the field expands with each new discovery, the PSRSIGSIM will also be a useful tool for educating and teaching students and bringing new people into the field of pulsar and GW astronomy. We are truly entering a new age of GW astronomy where a detection of the nanohertz SGWB is within reach, and as with each new window that opens in astronomy, there are many exciting discoveries to made. 


\section{Appendix A}

\section{Binary Orbital Parameters}

While our formalism for the short timescale timing model as described in $§ 3.3 .1$ is the same as is used in Lam et al. (2016a), we have assumed that the fit to the timing residuals is well described by a quadratic as shown in Eq. 3.3. However, for MSPs in a binary system, the observed pulse period can be Doppler shifted by some amount (Lorimer \& Kramer, 2004),

$$
\begin{aligned}
\sigma_{P_{b}} \sim P \frac{\delta \nu_{\|}}{c} \sim & \frac{2 \pi P}{c} \delta\left(\frac{a \sin i}{P_{b}}\right) \sim \frac{2 \pi P a \sin i}{c P_{b}} \sqrt{\left(\frac{\delta a}{a}\right)^{2}+\left(\frac{\delta \sin i}{\sin i}\right)^{2}+\left(\frac{\delta P_{b}}{P_{b}}\right)^{2}} \\
& \sim 72.7 \mathrm{~ns} P_{\mathrm{ms}} a_{\mathrm{lsec}} \sin i P_{b, \text { day }}^{-1} \sqrt{\left(\frac{\delta a}{a}\right)^{2}+\left(\frac{\delta \sin i}{\sin i}\right)^{2}+\left(\frac{\delta P_{b}}{P_{b}}\right)^{2}} .
\end{aligned}
$$

Here $a$ is the semimajor axis, $i$ is the inclination angle, and $P_{b}$ is the binary orbital period, and we assume that the errors on the binary parameters are uncorrelated.

However, the error induced by this in the timing residuals that are fit for in Eq. 3.2 will follow a cubic of $\sim \sigma_{P_{b}}\left(T / P_{b}\right)^{3}$, where $T$ is the length of the observation. Due to the length of the observations used in this analysis, $\left(T / P_{b}\right)$ may be quite large. In fact, for PSR J0023+0923, the MSP with the shortest binary period in this work, $\sim 200$ minutes, $\left(T / P_{b}\right)=0.855$. However, for PSR J0023+0923, $\sigma_{P_{b}} \approx 1 \times 10^{-4} \mathrm{~ns}$, so the total error is $\ll 1$ ns. Out of all pulsars in this work, the largest binary parameter error is for PSR J0613-0200 of 0.08 ns. As this is much less than $\sigma_{\mathcal{R}}$ for 
all of the observations in this work, the error induced by the binary orbit parameters is negligible. 


\section{Bibliography}

Abbott, B. P., Abbott, R., Abbott, T. D., et al. 2016, Physical Review Letters, 116, 061102, doi: http://doi.org/10.1103/PhysRevLett.116.06110210.1103/PhysRevLett.116.061102

—. 2017a, Phys. Rev. Lett., 119, 161101, doi: http://doi.org/10.1103/PhysRevLett.119.16110110.11

-. 2017b, ApJL, 848, L12, doi: http://doi.org/10.3847/20418213/aa91c910.3847/2041-8213/aa91c9

Ables, J. G., \& Manchester, R. N. 1976, A\&A, 50, 177

Aggarwal, K., Arzoumanian, Z., Baker, P. T., et al. 2019, ApJ, 880, 116, doi: http://doi.org/10.3847/1538-4357/ab223610.3847/1538-4357/ab2236

Alam, M. F., Arzoumanian, Z., Baker, P. T., et al. 2020a, arXiv e-prints, arXiv:2005.06495. https://arxiv.org/abs/2005.06495https://arxiv.org/abs/2005.06495

—. 2020b, arXiv e-prints, arXiv:2005.06490. https://arxiv.org/abs/2005.06490https://arxiv.org/ab

—. 2020c, arXiv e-prints, arXiv:2005.06495. https://arxiv.org/abs/2005.06495https://arxiv.org/ab

—. 2021, ApJS, 252, 4, doi: http://doi.org/10.3847/1538-4365/abc6a010.3847/1538$4365 / \mathrm{abc} 6 \mathrm{a} 0$

Aldabergenov, Y., Addazi, A., \& Ketov, S. V. 2021, Physics Letters B, 814, 136069, doi: http://doi.org/10.1016/j.physletb.2021.13606910.1016/j.physletb.2021.136069 
Antoniadis, J., Freire, P. C. C., Wex, N., et al. 2013, Science, 340, 448, doi: http://doi.org/10.1126/science.123323210.1126/science.1233232

Archibald, A. M., Gusinskaia, N. V., Hessels, J. W. T., et al. 2018, Nature, 559, 73, doi: http://doi.org/10.1038/s41586-018-0265-110.1038/s41586-018-0265-1

Arzoumanian, Z., Brazier, A., Burke-Spolaor, S., et al. 2015, ApJ, 813, 65, doi: http://doi.org/10.1088/0004-637X/813/1/6510.1088/0004-637X/813/1/65

-. 2016, ApJ, 821, 13, doi: http://doi.org/10.3847/0004$637 \mathrm{X} / 821 / 1 / 1310.3847 / 0004-637 \mathrm{X} / 821 / 1 / 13$

Arzoumanian, Z., Baker, P. T., Brazier, A., et al. 2018a, ApJ, 859, 47, doi: http://doi.org/10.3847/1538-4357/aabd3b10.3847/1538-4357/aabd3b

-. 2018b, ApJ, 859, 47, doi: http://doi.org/10.3847/15384357/aabd3b10.3847/1538-4357/aabd3b

Arzoumanian, Z., Brazier, A., Burke-Spolaor, S., et al. 2018c, ApJS, 235, 37, doi: http://doi.org/10.3847/1538-4365/aab5b010.3847/1538-4365/aab5b0

Arzoumanian, Z., Baker, P. T., Brazier, A., et al. 2020a, ApJ, 900, 102, doi: http://doi.org/10.3847/1538-4357/ababa110.3847/1538-4357/ababa1

Arzoumanian, Z., Baker, P. T., Blumer, H., et al. 2020b, arXiv e-prints, arXiv:2009.04496. https://arxiv.org/abs/2009.04496https://arxiv.org/abs/2009.04496 
Astropy Collaboration, Robitaille, T. P., Tollerud, E. J., et al. 2013, A\&A, 558, A33, doi: http://doi.org/10.1051/0004-6361/20132206810.1051/0004-6361/201322068

Babak, S., Petiteau, A., Sesana, A., et al. 2016, MNRAS, 455, 1665, doi: http://doi.org/10.1093/mnras/stv209210.1093/mnras/stv2092

Backer, D. C. 1970, Nature, 228, 42, doi: http://doi.org/10.1038/228042a010.1038/228042a0

Backer, D. C., Kulkarni, S. R., Heiles, C., Davis, M. M., \& Goss, W. M. 1982, Nature, 300, 615, doi: http://doi.org/10.1038/300615a010.1038/300615a0

Bailes, M., Barr, E., Bhat, N. D. R., et al. 2016, in MeerKAT Science: On the Pathway to the SKA, 11. https://arxiv.org/abs/1803.07424https://arxiv.org/abs/1803.07424

Bates, S. D., Lorimer, D. R., \& Verbiest, J. P. W. 2013, MNRAS, 431, 1352, doi: http://doi.org/10.1093/mnras/stt25710.1093/mnras/stt257

Bhandari, S., Keane, E. F., Barr, E. D., et al. 2018, MNRAS, 475, 1427, doi: http://doi.org/10.1093/mnras/stx307410.1093/mnras/stx3074

Bhat, N. D. R., Cordes, J. M., Camilo, F., Nice, D. J., \& Lorimer, D. R. 2004, ApJ, 605, 759, doi: http://doi.org/10.1086/38268010.1086/382680

Bhat, N. D. R., Ord, S. M., Tremblay, S. E., McSweeney, S. J., \& Tingay, S. J. 2016, ApJ, 818, 86, doi: http://doi.org/10.3847/0004-637X/818/1/8610.3847/0004$637 \mathrm{X} / 818 / 1 / 86$ 
Bhattacharya, D., \& van den Heuvel, E. P. J. 1991, Phys. Rep, 203, 1, doi: http://doi.org/10.1016/0370-1573(91)90064-S10.1016/0370-1573(91)90064-S

Bhattacharyya, B., Lyne, A. G., Stappers, B. W., et al. 2018, MNRAS, 477, 4090, doi: http://doi.org/10.1093/mnras/sty92310.1093/mnras/sty923

Bracewell, R. N. 1999

Brook, P. R., Karastergiou, A., McLaughlin, M. A., et al. 2018, ApJ, 868, 122, doi: http://doi.org/10.3847/1538-4357/aae9e310.3847/1538-4357/aae9e3

Burke-Spolaor, S. 2013, in Neutron Stars and Pulsars: Challenges and Opportunities after 80 years, ed. J. van Leeuwen, Vol. 291, 95-100, doi: http://doi.org/10.1017/S174392131202327710.1017/S1743921312023277

Burke-Spolaor, S., Johnston, S., Bailes, M., et al. 2012, MNRAS, 423, 1351, doi: http://doi.org/10.1111/j.1365-2966.2012.20998.x10.1111/j.13652966.2012.20998.x

Burke-Spolaor, S., Taylor, S. R., Charisi, M., et al. 2019, A\&A Rev., 27, 5, doi: http://doi.org/10.1007/s00159-019-0115-710.1007/s00159-019-0115-7

Camilo, F., Reynolds, J., Johnston, S., Halpern, J. P., \& Ransom, S. M. 2008, ApJ, 679, 681, doi: http://doi.org/10.1086/58705410.1086/587054

Chatterjee, S., Law, C. J., Wharton, R. S., et al. 2017, Nature, 541, 58, doi: http://doi.org/10.1038/nature2079710.1038/nature20797 
Chen, J. L., \& Wang, H. G. 2014, ApJS, 215, 11, doi: http://doi.org/10.1088/00670049/215/1/1110.1088/0067-0049/215/1/11

Coles, W. A., Kerr, M., Shannon, R. M., et al. 2015, ApJ, 808, 113, doi: http://doi.org/10.1088/0004-637X/808/2/11310.1088/0004-637X/808/2/113

Cordes, J. M. 1978, ApJ, 222, 1006, doi: http://doi.org/10.1086/15621810.1086/156218

—. 1986, ApJ, 311, 183, doi: http://doi.org/10.1086/16476410.1086/164764

Cordes, J. M. 2002, in Astronomical Society of the Pacific Conference Series, Vol. 278, Single-Dish Radio Astronomy: Techniques and Applications, ed. S. Stanimirovic, D. Altschuler, P. Goldsmith, \& C. Salter, 227-250

- 2013, Classical and Quantum Gravity, 30, 224002, doi: $\quad$ http://doi.org/10.1088/0264-9381/30/22/22400210.1088/0264$9381 / 30 / 22 / 224002$

Cordes, J. M., Bhat, N. D. R., Hankins, T. H., McLaughlin, M. A., \& Kern, J. 2004, ApJ, 612, 375, doi: http://doi.org/10.1086/42249510.1086/422495

Cordes, J. M., \& Downs, G. S. 1985, ApJS, 59, 343, doi: http://doi.org/10.1086/19107610.1086/191076

Cordes, J. M., \& Lazio, T. J. W. 2002, ArXiv Astrophysics e-prints

Cordes, J. M., Pidwerbetsky, A., \& Lovelace, R. V. E. 1986, ApJ, 310, 737, doi: http://doi.org/10.1086/16472810.1086/164728 
Cordes, J. M., \& Rickett, B. J. 1998, ApJ, 507, 846, doi: http://doi.org/10.1086/30635810.1086/306358

Cordes, J. M., Rickett, B. J., Stinebring, D. R., \& Coles, W. A. 2006, ApJ, 637, 346, doi: http://doi.org/10.1086/49833210.1086/498332

Cordes, J. M., \& Shannon, R. M. 2008, ApJ, 682, 1152, doi: http://doi.org/10.1086/58942510.1086/589425

—. 2010, arXiv e-prints, arXiv:1010.3785. https://arxiv.org/abs/1010.3785https://arxiv.org/abs/1

Cordes, J. M., Shannon, R. M., \& Stinebring, D. R. 2016, ApJ, 817, 16, doi: http://doi.org/10.3847/0004-637X/817/1/1610.3847/0004-637X/817/1/16

Cordes, J. M., Wolszczan, A., Dewey, R. J., Blaskiewicz, M., \& Stinebring, D. R. 1990, ApJ, 349, 245, doi: http://doi.org/10.1086/16831010.1086/168310

Cromartie, H. T., Fonseca, E., Ransom, S. M., et al. 2020, Nature Astronomy, 4, 72, doi: http://doi.org/10.1038/s41550-019-0880-210.1038/s41550-019-0880-2

Cui, B.-Y., Boyles, J., McLaughlin, M. A., \& Palliyaguru, N. 2017, ApJ, 840, 5, doi: http://doi.org/10.3847/1538-4357/aa6aa910.3847/1538-4357/aa6aa9

Dai, S., Hobbs, G., Manchester, R. N., et al. 2015, MNRAS, 449, 3223, doi: http://doi.org/10.1093/mnras/stv50810.1093/mnras/stv508

Demorest, P. B. 2007, PhD thesis, University of California, Berkeley

- 2018, nanopipe: Calibration and data reduction pipeline for pulsar timing. http://ascl.net/1803.004http://ascl.net/1803.004 
Demorest, P. B., Ferdman, R. D., Gonzalez, M. E., et al. 2013, ApJ, 762, 94, doi: http://doi.org/10.1088/0004-637X/762/2/9410.1088/0004-637X/762/2/94

Dolch, T., Lam, M. T., Cordes, J., et al. 2014, ApJ, 794, 21, doi: http://doi.org/10.1088/0004-637X/794/1/2110.1088/0004-637X/794/1/21

Dolch, T., NANOGrav Collaboration, Ellis, J. A., et al. 2016, in Journal of Physics Conference Series, Vol. 716, Journal of Physics Conference Series, 012014, doi: http://doi.org/10.1088/1742-6596/716/1/01201410.1088/1742$6596 / 716 / 1 / 012014$

Dolch, T., Stinebring, D. R., Jones, G., et al. 2020, arXiv e-prints, arXiv:2008.10562. https://arxiv.org/abs/2008.10562https://arxiv.org/abs/2008.10562

Downs, G. S., \& Reichley, P. E. 1983, ApJS, 53, 169, doi: http://doi.org/10.1086/19089010.1086/190890

Dror, J. A., Hiramatsu, T., Kohri, K., Murayama, H., \& White, G. 2020, Phys. Rev. Lett., 124, 041804, doi: http://doi.org/10.1103/PhysRevLett.124.04180410.1103/PhysRevLett.124.041804

DuPlain, R., Ransom, S., Demorest, P., et al. 2008, in Proc. SPIE, Vol. 7019, Advanced Software and Control for Astronomy II, 70191D, doi: http://doi.org/10.1117/12.79000310.1117/12.790003

Einstein, A. 1915, Sitzungsber. preuss.Akad. Wiss., vol. 47, No.2, pp. 831-839, 1915, 47, 831 
Farah, W., Flynn, C., Bailes, M., et al. 2018, MNRAS, 478, 1209, doi: http://doi.org/10.1093/mnras/sty112210.1093/mnras/sty1122

Ford, J. M., Demorest, P., \& Ransom, S. 2010, Society of PhotoOptical Instrumentation Engineers (SPIE) Conference Series, Vol. 7740, Heterogeneous real-time computing in radio astronomy, 77400A, doi: http://doi.org/10.1117/12.85766610.1117/12.857666

Freire, P. C. C., \& Tauris, T. M. 2014, MNRAS, 438, L86, doi: http://doi.org/10.1093/mnrasl/slt16410.1093/mnrasl/slt164

Gajjar, V., Siemion, A. P. V., Price, D. C., et al. 2018, ArXiv e-prints. https://arxiv.org/abs/1804.04101https://arxiv.org/abs/1804.04101

Gangadhara, R. T., \& Gupta, Y. 2001, ApJ, 555, 31, doi: http://doi.org/10.1086/32143910.1086/321439

Gersbach, K., \& Hazboun, J. S. 2019, in American Astronomical Society Meeting Abstracts, Vol. 233, American Astronomical Society Meeting Abstracts \#233, 149.22

Ghez, A. M., Klein, B. L., Morris, M., \& Becklin, E. E. 1998, ApJ, 509, 678, doi: http://doi.org/10.1086/30652810.1086/306528

Ginzburg, V. L., \& Zhelezniakov, V. V. 1975, ARA\&A, 13, 511, doi: http://doi.org/10.1146/annurev.aa.13.090175.00245510.1146/annurev.aa.13.090175.002455 
Goldreich, P., \& Julian, W. H. 1969, ApJ, 157, 869, doi: http://doi.org/10.1086/15011910.1086/150119

Hancock, P. J., Charlton, E. G., Macquart, J.-P., \& Hurley-Walker, N. 2019, arXiv e-prints, arXiv:1907.08395. https://arxiv.org/abs/1907.08395https://arxiv.org/abs/1907.08395

Hankins, T. H., Eilek, J. A., \& Jones, G. 2016, ApJ, 833, 47, doi: http://doi.org/10.3847/1538-4357/833/1/4710.3847/1538-4357/833/1/47

Haslam, C. G. T., Klein, U., Salter, C. J., et al. 1981, A\&A, 100, 209

Hazboun, J. S. 2020, Pulsar Data Toolbox, 0.2.2, Zenodo, doi: http://doi.org/10.5281/zenodo.408866010.5281/zenodo.4088660

Hazboun, J. S., Romano, J. D., \& Smith, T. L. 2019, Phys. Rev. D, 100, 104028, doi: http://doi.org/10.1103/PhysRevD.100.10402810.1103/PhysRevD.100.104028

Hazboun, J. S., Shapiro-Albert, B. J., Baker, P. T., et al. 2020, in prep

Helfand, D. J., Manchester, R. N., \& Taylor, J. H. 1975, ApJ, 198, 661, doi: http://doi.org/10.1086/15364410.1086/153644

Hellings, R. W., \& Downs, G. S. 1983, ApJL, 265, L39, doi: http://doi.org/10.1086/18395410.1086/183954

Hemberger, D. A., \& Stinebring, D. R. 2008, ApJL, 674, L37, doi: http://doi.org/10.1086/52898510.1086/528985 
Hessels, J. W. T., Ransom, S. M., Stairs, I. H., et al. 2006, Science, 311, 1901, doi: http://doi.org/10.1126/science.112343010.1126/science.1123430

Hewish, A. 1980, MNRAS, 192, 799, doi: http://doi.org/10.1093/mnras/192.4.79910.1093/mnras/1

Hewish, A., Bell, S. J., Pilkington, J. D. H., Scott, P. F., \& Collins, R. A. 1968, Nature, 217, 709, doi: http://doi.org/10.1038/217709a010.1038/217709a0

Hobbs, G. 2013, Classical and Quantum Gravity, 30, 224007, doi: $\quad$ http://doi.org/10.1088/0264-9381/30/22/22400710.1088/0264$9381 / 30 / 22 / 224007$

Hotan, A. W., van Straten, W., \& Manchester, R. N. 2004, PASA, 21, 302, doi: http://doi.org/10.1071/AS0402210.1071/AS04022

Hulse, R. A., \& Taylor, J. H. 1975, ApJL, 195, L51, doi: http://doi.org/10.1086/18170810.1086/181708

Jankowski, F., van Straten, W., Keane, E. F., et al. 2018, MNRAS, 473, 4436, doi: http://doi.org/10.1093/mnras/stx247610.1093/mnras/stx2476

Jiang, M., Cui, B.-Y., Schmid, N. A., McLaughlin, M. A., \& Cao, Z.-C. 2017, ApJ, 847, 75, doi: http://doi.org/10.3847/1538-4357/aa88c310.3847/1538-4357/aa88c3

Jones, E., Oliphant, T., Peterson, P., et al. 2001, SciPy: Open source scientific tools for Python. http://www.scipy.org/

Jones, M. L. 2018, PhD thesis, West Virginia University 
Jones, M. L., McLaughlin, M. A., Lam, M. T., et al. 2017, ApJ, 841, 125, doi: http://doi.org/10.3847/1538-4357/aa73df10.3847/1538-4357/aa73df

Joshi, B. C., Arumugasamy, P., Bagchi, M., et al. 2018, Journal of Astrophysics and Astronomy, 39, 51, doi: http://doi.org/10.1007/s12036-018-9549y10.1007/s12036-018-9549-y

Karastergiou, A., Hotan, A. W., van Straten, W., McLaughlin, M. A., \& Ord, S. M. 2009, MNRAS, 396, L95, doi: http://doi.org/10.1111/j.17453933.2009.00671.x10.1111/j.1745-3933.2009.00671.x

Karuppusamy, R., Stappers, B. W., \& van Straten, W. 2010, A\&A, 515, A36, doi: http://doi.org/10.1051/0004-6361/20091372910.1051/0004-6361/200913729

Keane, E. F. 2016, MNRAS, 459, 1360, doi: http://doi.org/10.1093/mnras/stw76710.1093/mnras/s

Keane, E. F., Kramer, M., Lyne, A. G., Stappers, B. W., \& McLaughlin, M. A. 2011, MNRAS, 415, 3065, doi: http://doi.org/10.1111/j.13652966.2011.18917.x10.1111/j.1365-2966.2011.18917.x

Keane, E. F., \& Petroff, E. 2015, MNRAS, 447, 2852, doi: http://doi.org/10.1093/mnras/stu265010.1093/mnras/stu2650

Keith, M. J., Coles, W., Shannon, R. M., et al. 2013, MNRAS, 429, 2161, doi: http://doi.org/10.1093/mnras/sts48610.1093/mnras/sts486

Kirsten, F., Bhat, N. D. R., Meyers, B. W., et al. 2019, ApJ, 874, 179, doi: http://doi.org/10.3847/1538-4357/ab0c0510.3847/1538-4357/ab0c05 
Kitajima, N., Soda, J., \& Urakawa, Y. 2021, Phys. Rev. Lett., 126, 121301, doi: http://doi.org/10.1103/PhysRevLett.126.12130110.1103/PhysRevLett.126.121301

Kolmogorov, A. 1941, Akademiia Nauk SSSR Doklady, 30, 301

Kormendy, J., \& Ho, L. C. 2013, ARA\&A, 51, 511, doi: $\quad$ http://doi.org/10.1146/annurev-astro-082708-10181110.1146/annurevastro-082708-101811

Kormendy, J., \& Richstone, D. 1995, ARA\&A, 33, 581, doi: http://doi.org/10.1146/annurev.aa.33.090195.00305310.1146/annurev.aa.33.090195.003053

Kramer, M., \& Champion, D. J. 2013, Classical and Quantum Gravity, 30, 224009, doi: http://doi.org/10.1088/0264-9381/30/22/22400910.1088/0264$9381 / 30 / 22 / 224009$

Kramer, M., Karastergiou, A., Gupta, Y., et al. 2003, A\&A, 407, 655, doi: http://doi.org/10.1051/0004-6361:2003084210.1051/0004-6361:20030842

Kramer, M., Lange, C., Lorimer, D. R., et al. 1999, ApJ, 526, 957, doi: http://doi.org/10.1086/30804210.1086/308042

Kramer, M., Xilouris, K. M., Lorimer, D. R., et al. 1998, ApJ, 501, 270, doi: http://doi.org/10.1086/30579010.1086/305790

Kramer, M., Stairs, I. H., Manchester, R. N., et al. 2006, Science, 314, 97, doi: http://doi.org/10.1126/science.113230510.1126/science.1132305 
Lam, M. T. 2017, PyPulse: PSRFITS handler, Astrophysics Source Code Library. http://ascl.net/1706.011http://ascl.net/1706.011

—. 2018, ApJ, 868, 33, doi: http://doi.org/10.3847/1538-4357/aae53310.3847/1538$4357 /$ aae 533

Lam, M. T., Cordes, J. M., Chatterjee, S., et al. 2016b, ApJ, 821, 66, doi: http://doi.org/10.3847/0004-637X/821/1/6610.3847/0004-637X/821/1/66

Lam, M. T., McLaughlin, M. A., Cordes, J. M., Chatterjee, S., \& Lazio, T. J. W. 2018a, ApJ, 861, 12, doi: http://doi.org/10.3847/1538-4357/aac48d10.3847/1538$4357 / \operatorname{aac} 48 d$

Lam, M. T., Cordes, J. M., Chatterjee, S., et al. 2016a, ApJ, 819, 155, doi: http://doi.org/10.3847/0004-637X/819/2/15510.3847/0004-637X/819/2/155

Lam, M. T., Ellis, J. A., Grillo, G., et al. 2018b, ApJ, 861, 132, doi: http://doi.org/10.3847/1538-4357/aac77010.3847/1538-4357/aac770

Lam, M. T., McLaughlin, M. A., Arzoumanian, Z., et al. 2019, ApJ, 872, 193, doi: http://doi.org/10.3847/1538-4357/ab01cd10.3847/1538-4357/ab01cd

Lambert, H. C., \& Rickett, B. J. 1999, ApJ, 517, 299, doi: http://doi.org/10.1086/30718110.1086/307181

Law, C. J., Abruzzo, M. W., Bassa, C. G., et al. 2017, ApJ, 850, 76, doi: http://doi.org/10.3847/1538-4357/aa970010.3847/1538-4357/aa9700 
Lawson, K. D., Mayer, C. J., Osborne, J. L., \& Parkinson, M. L. 1987, MNRAS, 225, 307, doi: http://doi.org/10.1093/mnras/225.2.30710.1093/mnras/225.2.307

Leblond, L., Shlaer, B., \& Siemens, X. 2009, Phys. Rev. D, 79, 123519, doi: http://doi.org/10.1103/PhysRevD.79.12351910.1103/PhysRevD.79.123519

Lee, K. J. 2016, in Astronomical Society of the Pacific Conference Series, Vol. 502, Frontiers in Radio Astronomy and FAST Early Sciences Symposium 2015, ed. L. Qain \& D. Li, 19

Lentati, L., Kerr, M., Dai, S., et al. 2017, MNRAS, 468, 1474, doi: http://doi.org/10.1093/mnras/stx58010.1093/mnras/stx580

Lentati, L., Taylor, S. R., Mingarelli, C. M. F., et al. 2015, MNRAS, 453, 2576, doi: http://doi.org/10.1093/mnras/stv153810.1093/mnras/stv1538

Levin, L., McLaughlin, M. A., Jones, G., et al. 2016, ApJ, 818, 166, doi: http://doi.org/10.3847/0004-637X/818/2/16610.3847/0004-637X/818/2/166

Li, X.-D. 2006, ApJL, 646, L139, doi: http://doi.org/10.1086/50696210.1086/506962

Liu, K., Verbiest, J. P. W., Kramer, M., et al. 2011, MNRAS, 417, 2916, doi: http://doi.org/10.1111/j.1365-2966.2011.19452.x10.1111/j.13652966.2011.19452.x

Liu, K., Desvignes, G., Cognard, I., et al. 2014, MNRAS, 443, 3752, doi: http://doi.org/10.1093/mnras/stu142010.1093/mnras/stu1420 
Liu, K., Young, A., Wharton, R., et al. 2019, ApJL, 885, L10, doi: http://doi.org/10.3847/2041-8213/ab4da810.3847/2041-8213/ab4da8

Lorimer, D. R. 2011, SIGPROC: Pulsar Signal Processing Programs, Astrophysics Source Code Library. http://ascl.net/1107.016http://ascl.net/1107.016

Lorimer, D. R., Bailes, M., McLaughlin, M. A., Narkevic, D. J., \& Crawford, F. 2007, Science, 318, 777, doi: http://doi.org/10.1126/science.114753210.1126/science.1147532

Lorimer, D. R., \& Kramer, M. 2004, Handbook of Pulsar Astronomy

Lorimer, D. R., Yates, J. A., Lyne, A. G., \& Gould, D. M. 1995, MNRAS, 273, 411, doi: http://doi.org/10.1093/mnras/273.2.41110.1093/mnras/273.2.411

Luo, J., Ransom, S., Demorest, P., et al. 2020, arXiv e-prints, arXiv:2012.00074. https://arxiv.org/abs/2012.00074https://arxiv.org/abs/2012.00074

Lyne, A. G., McLaughlin, M. A., Keane, E. F., et al. 2009, MNRAS, 400, 1439, doi: http://doi.org/10.1111/j.1365-2966.2009.15668.x10.1111/j.13652966.2009.15668.x

Lyne, A. G., \& Smith, F. G. 1982, Nature, 298, 825, doi: http://doi.org/10.1038/298825a010.1038/298825a0

Lyubarsky, Y. 2014, MNRAS, 442, L9, doi: http://doi.org/10.1093/mnrasl/slu04610.1093/mnrasl/s

Lyutikov, M. 2002, ApJL, 580, L65, doi: http://doi.org/10.1086/34549310.1086/345493 
Madison, D. R., Cordes, J. M., Arzoumanian, Z., et al. 2019, ApJ, 872, 150, doi: http://doi.org/10.3847/1538-4357/ab01fd10.3847/1538-4357/ab01fd

Main, R. A., Sanidas, S. A., Antoniadis, J., et al. 2020, MNRAS, doi: http://doi.org/10.1093/mnras/staa295510.1093/mnras/staa2955

Manchester, R. N., Hobbs, G. B., Teoh, A., \& Hobbs, M. 2005, AJ, 129, 1993, doi: http://doi.org/10.1086/42848810.1086/428488

Manchester, R. N., \& IPTA. 2013, Classical and Quantum Gravity, 30, 224010, doi: http://doi.org/10.1088/0264-9381/30/22/22401010.1088/0264$9381 / 30 / 22 / 224010$

Manchester, R. N., Lyne, A. G., Camilo, F., et al. 2001, MNRAS, 328, 17, doi: http://doi.org/10.1046/j.1365-8711.2001.04751.x10.1046/j.13658711.2001.04751.x

Maron, O., Kijak, J., Kramer, M., \& Wielebinski, R. 2000, A\&AS, 147, 195, doi: http://doi.org/10.1051/aas:200029810.1051/aas:2000298

McLaughlin, M. A. 2013, Classical and Quantum Gravity, 30, 224008, doi: $\quad$ http://doi.org/10.1088/0264-9381/30/22/22400810.1088/0264$9381 / 30 / 22 / 224008$

McLaughlin, M. A., Lyne, A. G., Lorimer, D. R., et al. 2006, Nature, 439, 817, doi: http://doi.org/10.1038/nature0444010.1038/nature04440

McLaughlin, M. A., Lyne, A. G., Keane, E. F., et al. 2009, MNRAS, 
400, 1431, doi: http://doi.org/10.1111/j.1365-2966.2009.15584.x10.1111/j.13652966.2009.15584.x

Meyers, B. W., Tremblay, S. E., Bhat, N. D. R., et al. 2017, ArXiv e-prints. https://arxiv.org/abs/1709.03651https://arxiv.org/abs/1709.03651

Michilli, D., Seymour, A., Hessels, J. W. T., et al. 2018, ArXiv e-prints. https://arxiv.org/abs/1801.03965https://arxiv.org/abs/1801.03965

Mickaliger, M. B., McEwen, A. E., McLaughlin, M. A., \& Lorimer, D. R. 2018, ArXiv e-prints. https://arxiv.org/abs/1807.00143https://arxiv.org/abs/1807.00143

Mickaliger, M. B., McLaughlin, M. A., Lorimer, D. R., et al. 2012, ApJ, 760, 64, doi: http://doi.org/10.1088/0004-637X/760/1/6410.1088/0004-637X/760/1/64

Mikami, R., Asano, K., Tanaka, S. J., et al. 2016, ApJ, 832, 212, doi: http://doi.org/10.3847/0004-637X/832/2/21210.3847/0004-637X/832/2/212

Mingarelli, C. M. F., \& Mingarelli, A. B. 2018, Journal of Physics Communications, 2, 105002, doi: http://doi.org/10.1088/2399-6528/aae06d10.1088/2399$6528 /$ aae06d

Moore, C. J., Cole, R. H., \& Berry, C. P. L. 2015, Classical and Quantum Gravity, 32, 015014, doi: http://doi.org/10.1088/0264-9381/32/1/01501410.1088/0264$9381 / 32 / 1 / 015014$

Ng, C. 2018, in IAU Symposium, Vol. 337, Pulsar Astrophysics the Next Fifty 
Years, ed. P. Weltevrede, B. B. P. Perera, L. L. Preston, \& S. Sanidas, 179-182, doi: http://doi.org/10.1017/S174392131701063810.1017/S1743921317010638

Nice, D., Demorest, P., Stairs, I., et al. 2015, Tempo: Pulsar timing data analysis. http://ascl.net/1509.002http://ascl.net/1509.002

Oppermann, N., Yu, H.-R., \& Pen, U.-L. 2018, MNRAS, 475, 5109, doi: http://doi.org/10.1093/mnras/sty00410.1093/mnras/sty004

Palliyaguru, N. T., McLaughlin, M. A., Keane, E. F., et al. 2011, MNRAS, 417, 1871, doi: http://doi.org/10.1111/j.1365-2966.2011.19388.x10.1111/j.13652966.2011.19388.x

Pennucci, T. T. 2019, ApJ, 871, 34, doi: http://doi.org/10.3847/15384357/aaf6ef10.3847/1538-4357/aaf6ef

Pennucci, T. T., Demorest, P. B., \& Ransom, S. M. 2014, ApJ, 790, 93, doi: http://doi.org/10.1088/0004-637X/790/2/9310.1088/0004-637X/790/2/93

Pennucci, T. T., Possenti, A., Esposito, P., et al. 2015, ApJ, 808, 81, doi: http://doi.org/10.1088/0004-637X/808/1/8110.1088/0004-637X/808/1/81

Petroff, E., Barr, E. D., Jameson, A., et al. 2016, PASA, 33, e045, doi: http://doi.org/10.1017/pasa.2016.3510.1017/pasa.2016.35

Philippov, A., Uzdensky, D. A., Spitkovsky, A., \& Cerutti, B. 2019, ApJL, 876, L6, doi: http://doi.org/10.3847/2041-8213/ab159010.3847/2041-8213/ab1590 
Pitkin, M. 2018, Journal of Open Source Software, 3, 538, doi: http://doi.org/10.21105/joss.0053810.21105/joss.00538

Pol, N. S., Taylor, S. R., Kelley, L. Z., et al. 2020, arXiv e-prints, arXiv:2010.11950. https://arxiv.org/abs/2010.11950https://arxiv.org/abs/2010.11950

Popov, M. V., \& Stappers, B. 2007, A\&A, 470, 1003, doi: http://doi.org/10.1051/0004-6361:2006658910.1051/0004-6361:20066589

Price-Whelan, A. M., Sipőcz, B. M., Günther, H. M., et al. 2018, AJ, 156, 123, doi: http://doi.org/10.3847/1538-3881/aabc4f10.3847/1538-3881/aabc4f

Rajwade, K., Lorimer, D. R., \& Anderson, L. D. 2016, MNRAS, 455, 493, doi: http://doi.org/10.1093/mnras/stv233410.1093/mnras/stv2334

Rane, A., \& Loeb, A. 2016, ArXiv e-prints. https://arxiv.org/abs/1608.06952https://arxiv.org/abs/1608.06952

Rankin, J. M. 1983, ApJ, 274, 333, doi: http://doi.org/10.1086/16145010.1086/161450

Ransom, S., Brazier, A., Chatterjee, S., et al. 2019, in Bulletin of the American Astronomical Society, Vol. 51, 195. https://arxiv.org/abs/1908.05356https://arxiv.org/abs/1908.05356

Rasmussen, C. E., W. C. K. I. 2006, Gaussian Processes for Machine Learning, 1st edn. (The MIT Press)

Rathnasree, N., \& Rankin, J. M. 1995, ApJ, 452, 814, doi: http://doi.org/10.1086/17634910.1086/176349 
Reardon, D. J. 2018, PhD thesis, Monash Centre for Astrophysics (MoCA), School of Physics and Astronomy, Monash University, Victoria 3800, Australia

Reardon, D. J., Coles, W. A., Hobbs, G., et al. 2019, MNRAS, 485, 4389, doi: http://doi.org/10.1093/mnras/stz64310.1093/mnras/stz643

Rickett, B. J. 1977, ARA\&A, 15, 479, doi: http://doi.org/10.1146/annurev.aa.15.090177.00240310.1

Rickett, B. J., Coles, W. A., \& Bourgois, G. 1984, A\&A, 134, 390

Rickett, B. J., Coles, W. A., Nava, C. F., et al. 2014, ApJ, 787, 161, doi: http://doi.org/10.1088/0004-637X/787/2/16110.1088/0004-637X/787/2/161

Romero, G. E., del Valle, M. V., \& Vieyro, F. L. 2016, Phys. Rev. D, 93, 023001, doi: http://doi.org/10.1103/PhysRevD.93.02300110.1103/PhysRevD.93.023001

Rutledge, R. E. 2006, ArXiv Astrophysics e-prints

Scheuer, P. A. G. 1968, Nature, 218, 920, doi: http://doi.org/10.1038/218920a010.1038/218920a0

Shannon, R. M., \& Cordes, J. M. 2010, ApJ, 725, 1607, doi: $\quad$ http://doi.org/10.1088/0004-637X/725/2/160710.1088/0004$637 \mathrm{X} / 725 / 2 / 1607$

-. 2012, ApJ, 761, 64, doi: http://doi.org/10.1088/0004$637 \mathrm{X} / 761 / 1 / 6410.1088 / 0004-637 \mathrm{X} / 761 / 1 / 64$

Shannon, R. M., Ravi, V., Coles, W. A., et al. 2013, Science, 342, 334, doi: http://doi.org/10.1126/science.123801210.1126/science.1238012 
Shannon, R. M., Osłowski, S., Dai, S., et al. 2014, MNRAS, 443, 1463, doi: http://doi.org/10.1093/mnras/stu121310.1093/mnras/stu1213

Shannon, R. M., Ravi, V., Lentati, L. T., et al. 2015, Science, 349, 1522, doi: http://doi.org/10.1126/science.aab191010.1126/science.aab1910

Shapiro-Albert, B. J., McLaughlin, M. A., Lam, M. T., Cordes, J. M., \& Swiggum, J. K. 2020, ApJ, 890, 123, doi: http://doi.org/10.3847/1538$4357 / \mathrm{ab} 65 \mathrm{f} 810.3847 / 1538-4357 / \mathrm{ab} 65 \mathrm{f} 8$

Sieber, W. 1982, A\&A, 113, 311

Skipper, J., Lynch, R. S., White, S., \& Ransom, S. M. 2019, in American Astronomical Society Meeting Abstracts, Vol. 233, American Astronomical Society Meeting Abstracts \#233, 153.16

Snedecor, G. W., \& Cochran, W. G. 1989, Statistical Methods

Spitler, L. G., Scholz, P., Hessels, J. W. T., et al. 2016, Nature, 531, 202, doi: http://doi.org/10.1038/nature1716810.1038/nature17168

Spitler, L. G., Herrmann, W., Bower, G. C., et al. 2018, ArXiv e-prints. https://arxiv.org/abs/1807.03722https://arxiv.org/abs/1807.03722

Stinebring, D. R., McLaughlin, M. A., Cordes, J. M., et al. 2001, ApJL, 549, L97, doi: http://doi.org/10.1086/31913310.1086/319133

Stinebring, D. R., Rickett, B. J., \& Koch Ocker, S. 2019, ApJ, 870, 82, doi: http://doi.org/10.3847/1538-4357/aaef8010.3847/1538-4357/aaef80 
Stinebring, D. R., Smirnova, T. V., Hankins, T. H., et al. 2000, ApJ, 539, 300, doi: http://doi.org/10.1086/30920110.1086/309201

Stovall, K., Ray, P. S., Blythe, J., et al. 2015, ApJ, 808, 156, doi: http://doi.org/10.1088/0004-637X/808/2/15610.1088/0004-637X/808/2/156

Stovall, K., Freire, P. C. C., Chatterjee, S., et al. 2018, ApJL, 854, L22, doi: http://doi.org/10.3847/2041-8213/aaad0610.3847/2041-8213/aaad06

Tan, C. M., Bassa, C. G., Cooper, S., et al. 2018, ApJ, 866, 54, doi: http://doi.org/10.3847/1538-4357/aade8810.3847/1538-4357/aade88

Taylor, J. H. 1992, Philosophical Transactions of the Royal Society of London Series A, 341, 117, doi: http://doi.org/10.1098/rsta.1992.008810.1098/rsta.1992.0088

Turner, J. E., McLaughlin, M. A., Cordes, J. M., et al. 2020, in prep

Vachaspati, T., \& Vilenkin, A. 1985, Phys. Rev. D, 31, 3052, doi: http://doi.org/10.1103/PhysRevD.31.305210.1103/PhysRevD.31.3052

Vallisneri, M., Taylor, S. R., Simon, J., et al. 2020, ApJ, 893, 112, doi: http://doi.org/10.3847/1538-4357/ab7b6710.3847/1538-4357/ab7b67

Van Der Walt, S., Colbert, S. C., \& Varoquaux, G. 2011, Computing in Science \& Engineering, 13, 22

van Straten, W., Demorest, P., \& Oslowski, S. 2012, Astronomical Research and Technology, 9, 237. https://arxiv.org/abs/1205.6276https://arxiv.org/abs/1205.6276 
Verbiest, J. P. W., Lentati, L., Hobbs, G., et al. 2016, MNRAS, 458, 1267, doi: http://doi.org/10.1093/mnras/stw34710.1093/mnras/stw347

Vieyro, F. L., Romero, G. E., Bosch-Ramon, V., Marcote, B., \& del Valle, M. V. 2017, A\&A, 602, A64, doi: http://doi.org/10.1051/00046361/20173055610.1051/0004-6361/201730556

Walker, M. A., Demorest, P. B., \& van Straten, W. 2013, ApJ, 779, 99, doi: http://doi.org/10.1088/0004-637X/779/2/9910.1088/0004-637X/779/2/99

Wang, N., Manchester, R. N., \& Johnston, S. 2007, MNRAS, 377, 1383, doi: http://doi.org/10.1111/j.1365-2966.2007.11703.x10.1111/j.13652966.2007.11703.x

Waxman, E. 2017, ApJ, 842, 34, doi: http://doi.org/10.3847/15384357/aa713e10.3847/1538-4357/aa713e

Wolszczan, A., \& Frail, D. A. 1992, Nature, 355, 145, doi: http://doi.org/10.1038/355145a010.1038/355145a0

Yao, J. M., Manchester, R. N., \& Wang, N. 2017, ApJ, 835, 29, doi: http://doi.org/10.3847/1538-4357/835/1/2910.3847/1538-4357/835/1/29

Zhang, B., Harding, A. K., \& Muslimov, A. G. 2000, ApJL, 531, L135, doi: http://doi.org/10.1086/31254210.1086/312542

Zhu, W. W., Stairs, I. H., Demorest, P. B., et al. 2015, ApJ, 809, 41, doi: http://doi.org/10.1088/0004-637X/809/1/4110.1088/0004-637X/809/1/41 
Zhu, W. W., Desvignes, G., Wex, N., et al. 2019, MNRAS, 482, 3249, doi: http://doi.org/10.1093/mnras/sty290510.1093/mnras/sty2905

Zhu, X. J., Hobbs, G., Wen, L., et al. 2014, MNRAS, 444, 3709, doi: http://doi.org/10.1093/mnras/stu171710.1093/mnras/stu1717 